ORNL/TM-2004/292

\title{
Investigation of Energy-Efficient Supermarket Display Cases
}

\section{December, 2004}

Prepared by:

Foster Miller, Inc.

David H. Walker

Principal Investigator

Southern California Edison RTTC

Ramin T. Faramarzi

Principal Investigator

Oak Ridge National Lab

Van D. Baxter 


\section{DOCUMENT AVAILABILITY}

Reports produced after January 1, 1996, are generally available free via the U.S. Department of Energy (DOE) Information Bridge:

Web site: http://www.osti.gov/bridge

Reports produced before January 1, 1996, may be purchased by members of the public from the following source:

National Technical Information Service

5285 Port Royal Road

Springfield, VA 22161

Telephone: 703-605-6000 (1-800-553-6847)

TDD: 703-487-4639

Fax: 703-605-6900

E-mail: info@ntis.fedworld.gov

Web site: http://www.ntis.gov/support/ordernowabout.htm

Reports are available to DOE employees, DOE contractors, Energy Technology Data Exchange (ETDE) representatives, and International Nuclear Information System (INIS) representatives from the following source:

Office of Scientific and Technical Information

P.O. Box 62

Oak Ridge, TN 37831

Telephone: 865-576-8401

Fax: 865-576-5728

E-mail: reports@adonis.osti.gov

Web site: http://www.osti.gov/contact.html

This report was prepared as an account of work sponsored by an agency of the United States Government. Neither the United States government nor any agency thereof, nor any of their employees, makes any warranty, express or implied, or assumes any legal liability or responsibility for the accuracy, completeness, or usefulness of any information, apparatus, product, or process disclosed, or represents that its use would not infringe privately owned rights. Reference herein to any specific commercial product, process, or service by trade name, trademark, manufacturer, or otherwise, does not necessarily constitute or imply its endorsement, recommendation, or favoring by the United States Government or any agency thereof. The views and opinions of authors expressed herein do not necessarily state or reflect those of the United States Government or any agency thereof. 


\title{
INVESTIGATION OF ENERGY-EFFICIENT DISPLAY CASES
}

\author{
David H. Walker \\ Foster-Miller, Inc. \\ Ramin T. Faramarzi \\ Southern California Edison RTTC \\ Van D. Baxter \\ Oak Ridge National Lab
}

December 2004

\author{
Prepared for \\ OAK RIDGE NATIONAL LABORATORY \\ P.O. Box 2008 \\ Oak Ridge, Tennessee 37831-6285 \\ managed by \\ UT-Battelle, LLC \\ for the \\ U.S. DEPARTMENT OF ENERGY \\ under contract DE-AC05-00OR22725 \\ Subcontract No. 4000007074 \\ COTR: Van D. Baxter
}





\section{CONTENTS}

$\begin{array}{ll}\text { Section } & \text { Page }\end{array}$

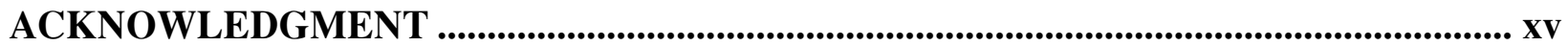

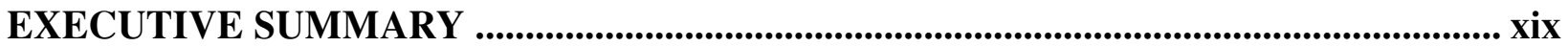

1. INTRODUCTION _.............................................................................................................. 1

2. SUPERMARKET REFRIGERATION AND DISPLAY

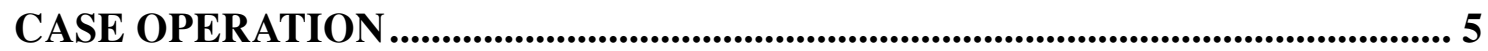

3. SURVEY OF PRESENT SUPERMARKET DISPLAY CASES ................................ 12

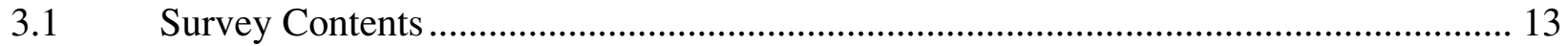

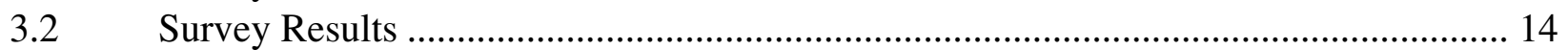

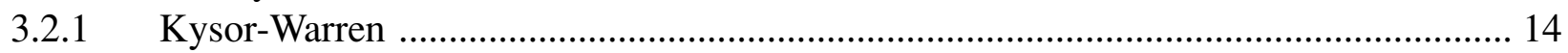

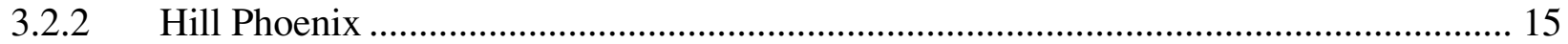

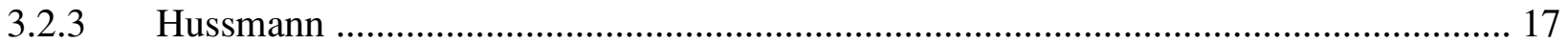

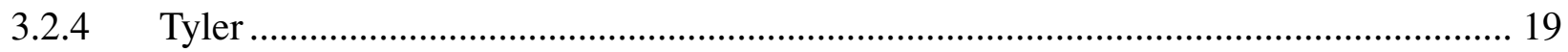

4. RESULTS FROM THE PROJECT WORKSHOP AND

4.1 Initial Project Workshop …………………....................................................... 21

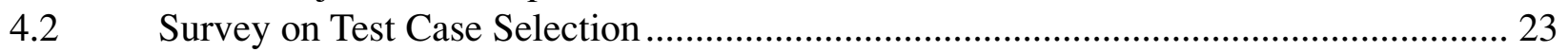

4.3 Survey from the Food Marketing Institute (FMI) Energy Conference ............................ 24

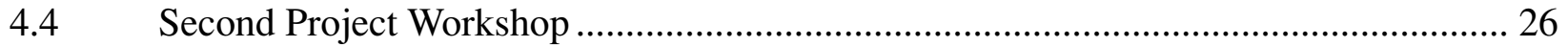

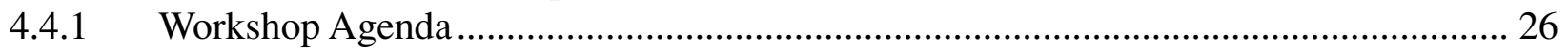

4.4.2 Case Efficiency Improvement Technology Selection and Energy Analysis

Results (Ramin Faramarzi - Southern California Edison RTTC) ................................... 26

4.4.3 Draft Rating Procedure for High-Efficiency Display Cases

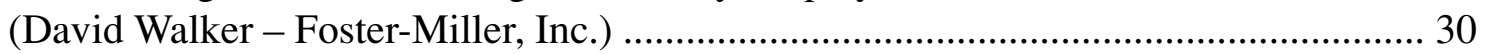

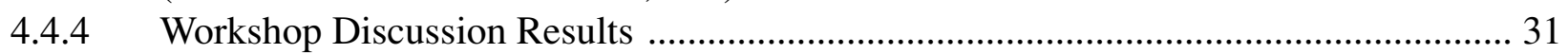

4.4.5 Attendees List .................................................................................................. 33

4.5 Third Project Workshop - Southern California Edison, Irwindale, CA -

January 22, 2004 ...................................................................................................... 33

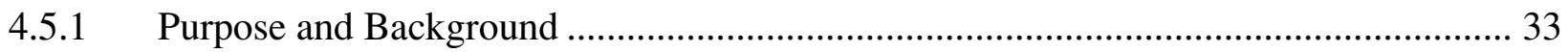


4.5.2 Case Efficiency Improvement Technology Selection and Energy

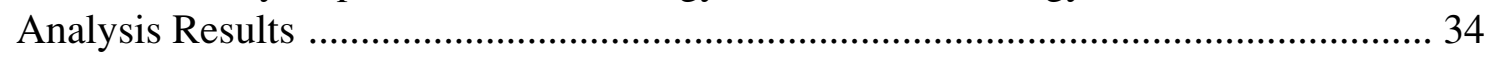

4.5.3 Discussion Session ........................................................................................... 35

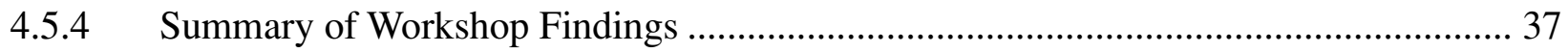

5. SELECTION OF ENERGY EFFICIENCY MEASURES ................................... 39

EEMs Descriptions and Categories .................................................................... 40

6. INVESTIGATION OF DISPLAY CASE PERFORMANCE .............................. 44

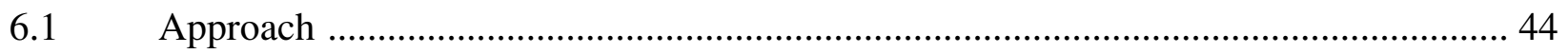

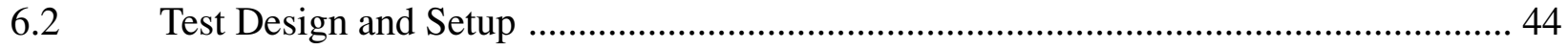

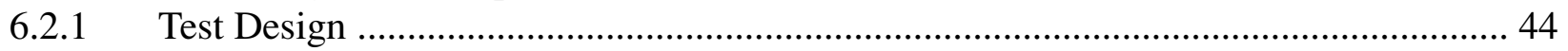

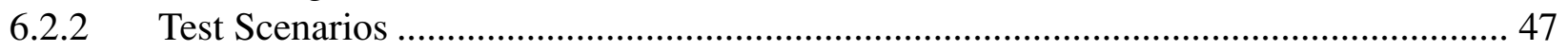

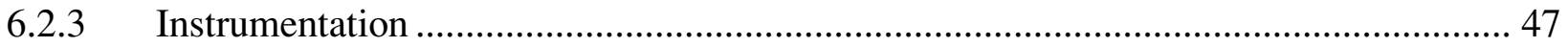

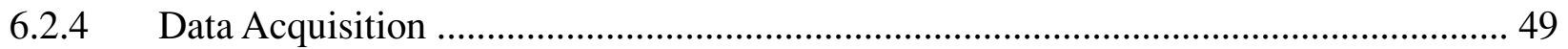

6.2.5 Controlled Environment Room Condition ............................................................... 50

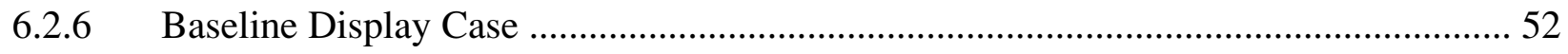

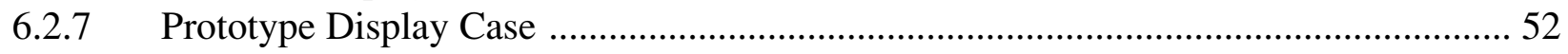

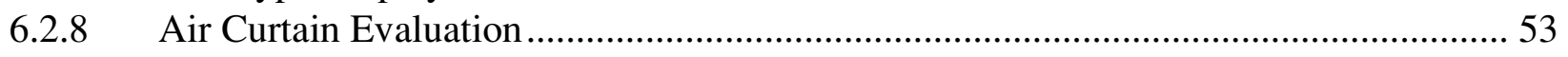

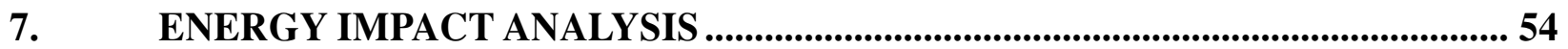

7.1 A Brief Description of the DOE-2.3 Simulation Program ....................................... 54

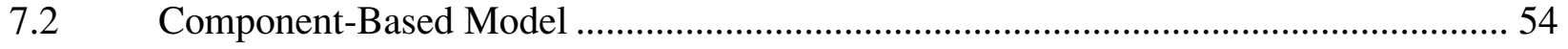

7.3 Refrigeration System Components ..................................................................... 55

7.4 Description of the Modeled Supermarket Site ................................................. 56

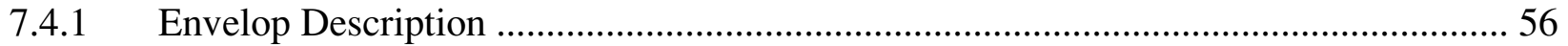

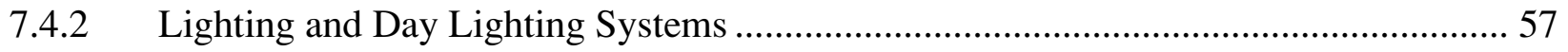

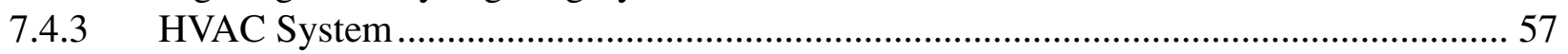

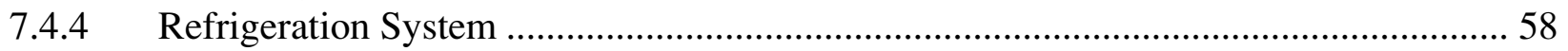

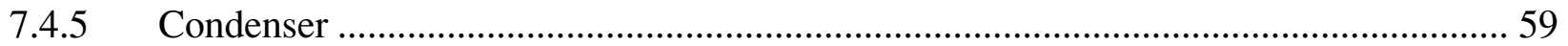

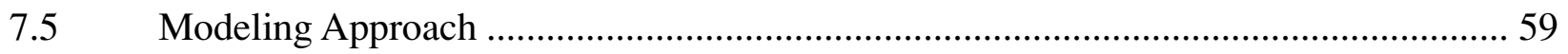

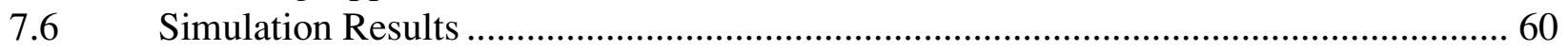

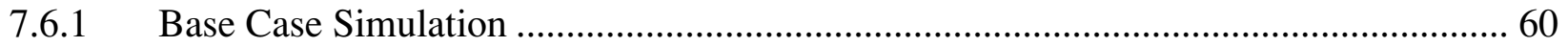

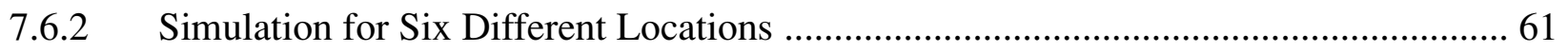

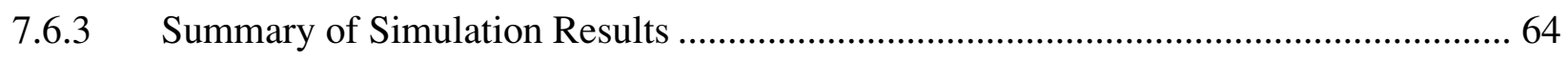




\section{INVESTIGATION OF AMBIENT AIR ENTRAINMENT IN THE} DISPLAY CASE AIR CURTAIN ......................................................................67

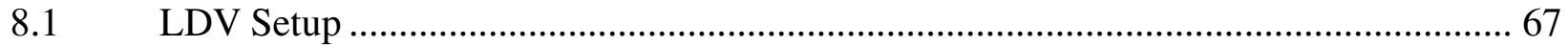

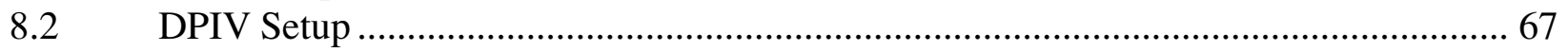

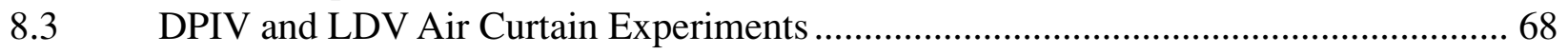

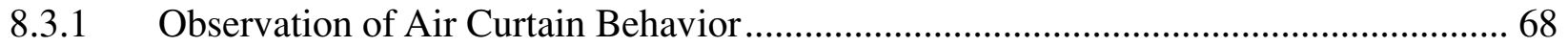

8.3.2 Measurement of DAG and RAG Air Flow Rates .......................................................... 69

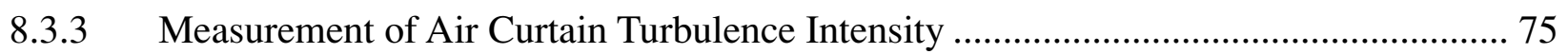

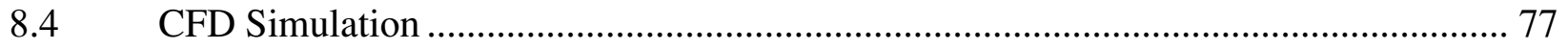

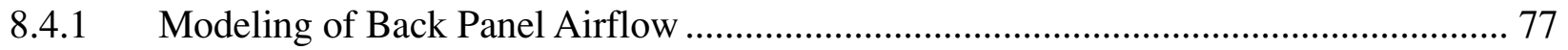

8.4.2 Modeling of Air Curtain Air Entrainment ………...................................................... 79

9. DISPLAY CASE TEST DATA THERMAL ANALYSIS APPROACH .................... 86

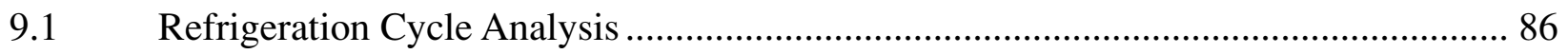

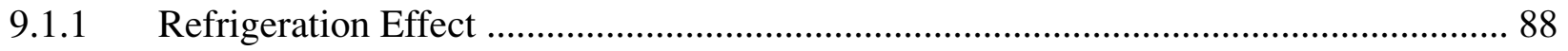

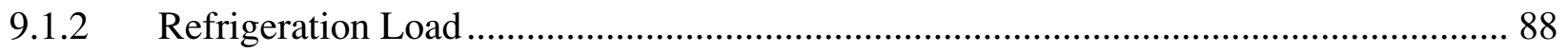

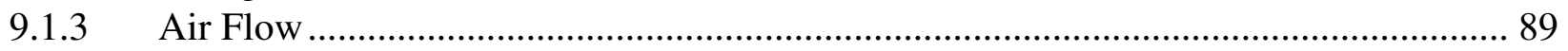

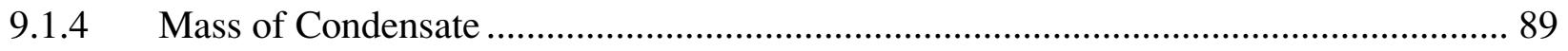

9.1.5 Latent and Sensible Loads ………………............................................................. 90

9.1.6 Cooling Load Based on One Running Cycle ……………............................................ 91

9.1.7 Cooling Load Using Psychrometric Data .................................................................. 91

9.1.8 Evaporator Coil Characteristic Performance ………….................................................. 92

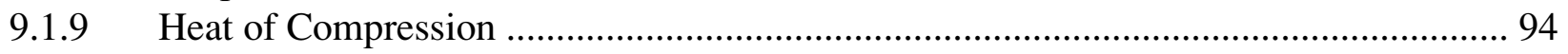

9.1.10 Total System Power and Energy ……………............................................................ 95

9.2 Display Case Heat Transfer Analysis ............................................................................. 96

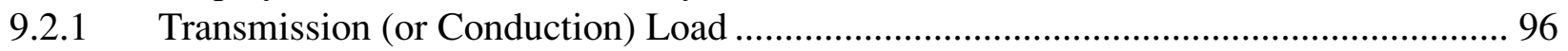

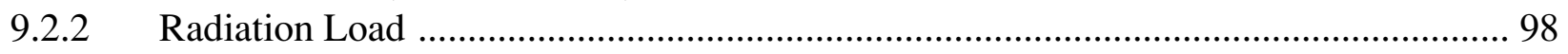

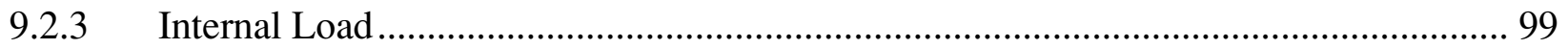

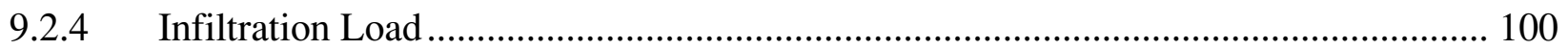

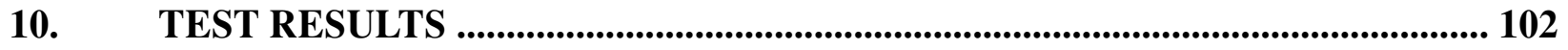

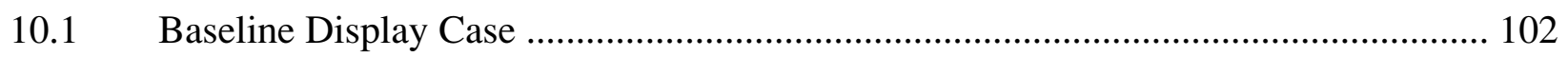

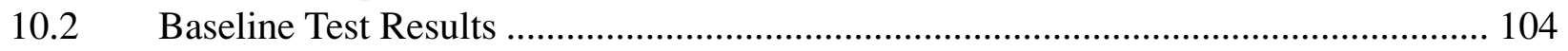

$10.3 \quad$ Prototype Test Results ....................................................................................... 121

10.3.1 Discharge Air Temperature and Product Temperatures ……………………................ 124

10.3.2 Collected Condensate and Defrost Duration .................................................................. 126

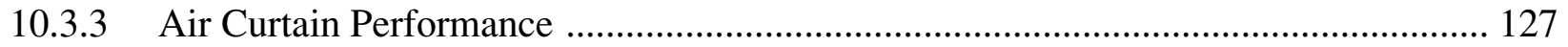

10.3.4 Evaporator Coil Effectiveness, Superheat, and Total Subcooling .............................. 127 
10.3.5 Refrigeration Effect and Refrigerant Mass Flow Rate ......................................... 127

10.3.6 Cooling Capacity and Cooling Load Components ............................................... 128

10.3.7 Power, Energy, and System Efficiency ........................................................... 128

11. DEVELOPMENT OF A DRAFT RATING PROCEDURE FOR HIGHEFFICIENCY REFRIGERATED DISPLAY CASES ....................................... 130

12. CONCLUSIONS AND RECOMMENDATIONS ........................................ 137

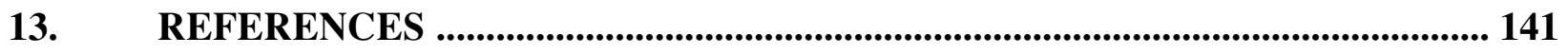




\section{ILLUSTRATIONS}

Figure

Page

1. Layout of the refrigerated fixtures in a supermarket ................................................. 6

2. Display case types employed in supermarkets ........................................................ 7

3. Typical open, vertical multi-deck display case air circulation pattern ....................... 8

4. Percentage breakdown of fixture lineal feet by type in a typical supermarket ............. 39

5. Refrigeration load percentage breakdown for meat, dairy/deli and frozen

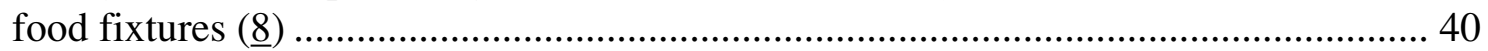

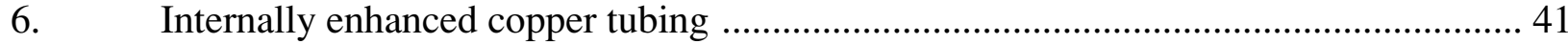

7. Photographs of the tangential fans and evaporator fan motor ................................... 41

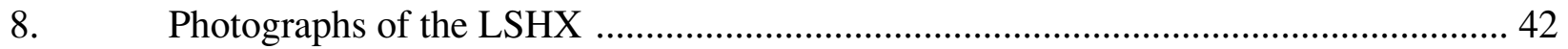

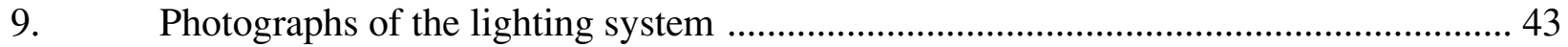

10. 1st generation improved geometry of the DAG for infiltration rate reduction ............. 43

11. Custom raised frame assembly and special drain piping/valve arrangement ............... 45

12. Location of product simulators inside the display case ........................................ 46

13. Simulated and dummy product used in the display case ..........................................46

14. Locations of sensors for open vertical multi-deck meat display case ......................... 49

15. Locations of sensors for evaporator coil of the display case .................................. 50

16. High precision digital scale used to measure the weight of condensate collected ........ 51

17. Schematic diagram of the air-conditioning and heating system of the RTTC's

controlled environment room ............................................................................. 51

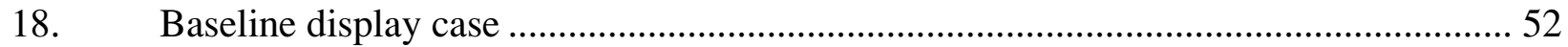

19. Schematic floor plan of the modeled supermarket .............................................. 57

20. Energy usage by end-use (six months field monitored data) ................................... 59

21. Monthly refrigeration and HVAC compressors energy usage

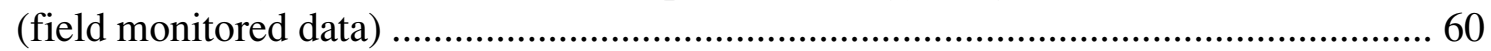

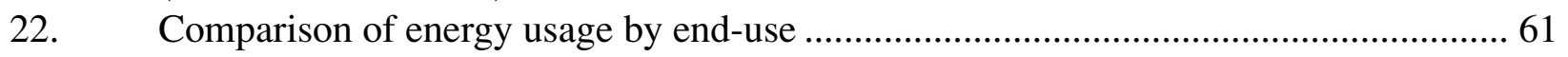

23. Impact of energy efficiency measures on total cooling load ................................... 62

24. Impact of energy efficiency measures on total refrigeration energy ......................... 62

25. Impact of energy efficiency measures on total building energy ................................ 63

26. Annual average wet-bulb and dry-bulb temperatures for all six locations ................... 63

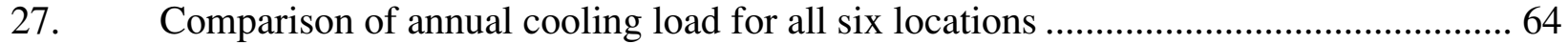

28. Comparison of annual refrigeration energy for all six locations .............................. 65

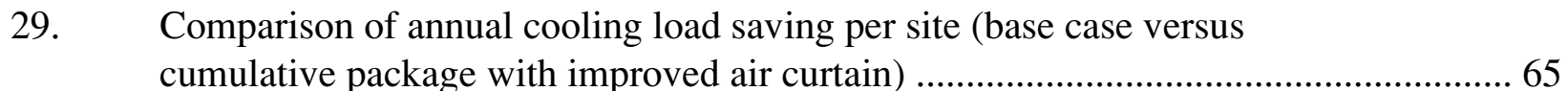

30. Comparison of annual cooling load saving per site (base case versus

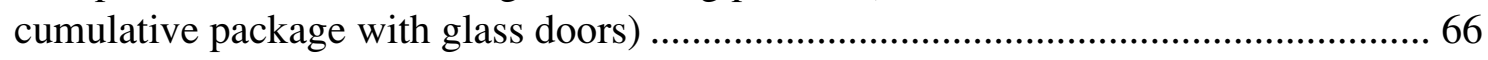




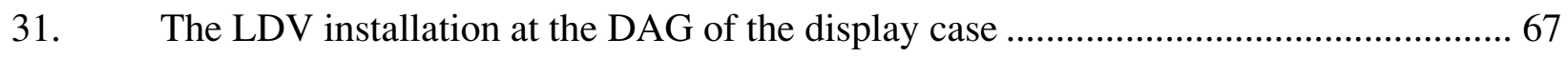

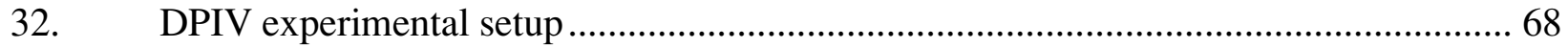

33. Vertical velocity field in the mid-plane of the display case by DPIV ........................ 69

34. The display case mid-plane streamlines at steady state by DPIV (dotted line represents the surface used for the determination of total flow of entrained ambient air in Equation 5)

35. Vertical velocity profile by LDV at two separate locations and by DPIV in the mid-plane of the display case. (The 0 in. DAG position in the figure corresponds

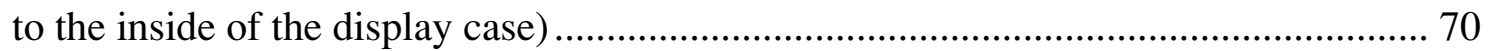

36. The vertical velocity component at the RAG by DPIV and LDV methods ................ 72

37. Nomenclature of the energy analysis used to determine the rate of air infiltration....... 73

38. Turbulence intensity at the DAG by LDV and DPIV methods ................................ 76

39. The vertical velocity component at the RAG by DPIV and LDV methods ................. 77

40. Comparison of CFD temperature field (left side) with infrared image (right side) ...... 78

41. (a) Streamlines for the display case predicted by the (CFluent code and temperature comparison with back panel flow present: (b) Temperature comparison with no back panel flow ................................................................. 79

42. Entrainment rate for the display case as a function of turbulence intensity

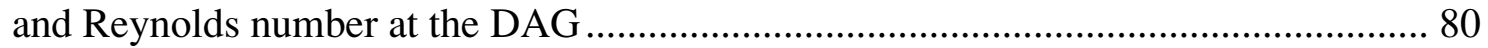

43. Schematic of velocity profiles under consideration for parametric studies .................. 81

44. Entrainment rate as a function of imposed turbulence intensity and different

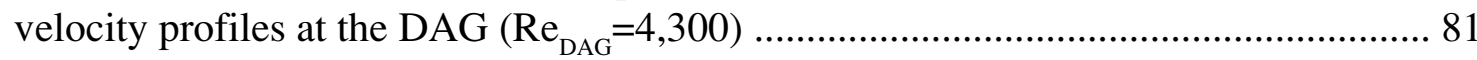

45. Turbulent kinetic energy contours and velocity vectors for a variety of geometries at the DAG.....

46. Vertical velocity profile at the DAG for the all cases depicted in 45 (NOTE: profiles from original baseline case DAG design and 20 deg-slanted surface design of prototype case are from measured data; profile from $57 \mathrm{deg}$-slanted surface is estimated from CFD modeling) .... 84

47. Entrainment rate as a function of turbulence intensity at the DAG for different

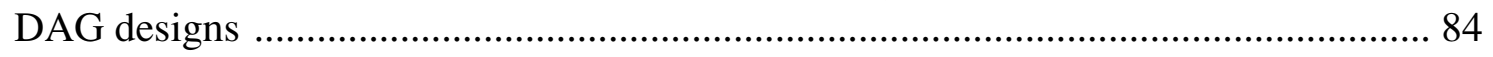

48. Variation of entrainment rate with DAG throw angle ......................................... 85

49. Schematics of inner and outer shell of the case and insulation between them ............. 97

50. Surfaces participating in radiation heat transfer for display case ............................. 99

51. Photograph of the four-deck deli display case ...................................................... 103

52. Schematic of the four-deck deli display case (courtesy of Hill Phoenix) .................. 103

53. 2 min profile of the controlled environment room DB and RH over $24 \mathrm{hr} . \ldots \ldots \ldots \ldots . . . . .104$

54. $2 \mathrm{~min}$ profile of suction and discharge pressures over $24 \mathrm{hr}$................................... 105

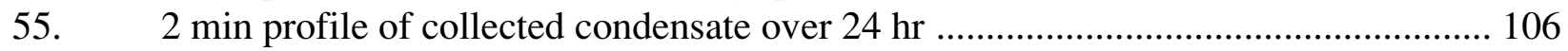

56. Breakdown of collected condensate over $24 \mathrm{hr}$................................................ 106

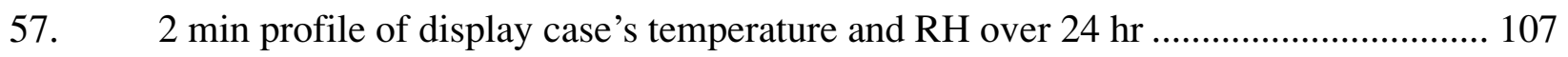


58. $2 \mathrm{~min}$ profile of pressure differential across the evaporator coil over $24 \mathrm{hr} \ldots \ldots \ldots \ldots . . .108$

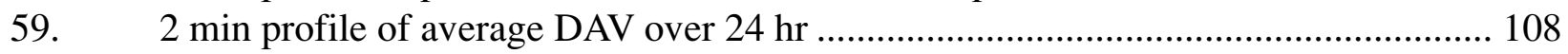

60. 2 min profile of DAV along the honeycomb (or DAG) over $24 \mathrm{hr}$......................... 109

61. Hourly profile of total cooling load per linear feet of the fixture over $24 \mathrm{hr} . \ldots \ldots \ldots . . . . .109$

62. Hourly profile of total, sensible and latent cooling load over $24 \mathrm{hr}$........................ 110

63. Total cooling load and its components using psychrometric and refrigeration

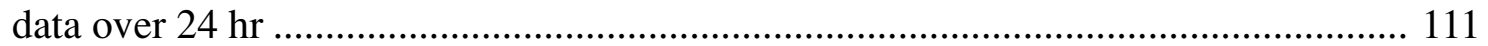

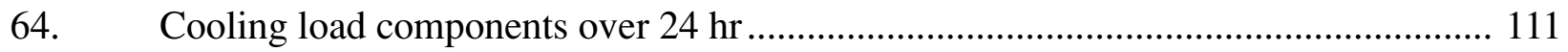

65. Percentage breakdown of the cooling load components over $24 \mathrm{hr}$......................... 112

66. Reduced cooling load, and average cooling load over $24 \mathrm{hr}$ and 3/4 of

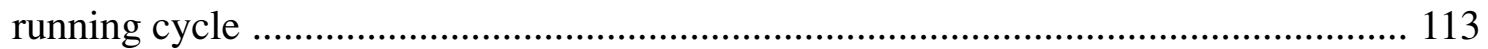

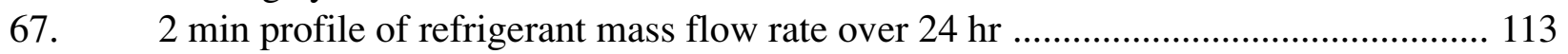

68. 2 min profiles of actual compressor power and refrigerant mass flow rate

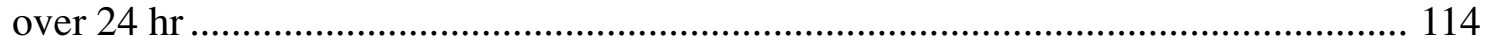

69. 2 min profile of average discharge air velocity and temperature over $24 \mathrm{hr} . \ldots \ldots \ldots \ldots . . . .114$

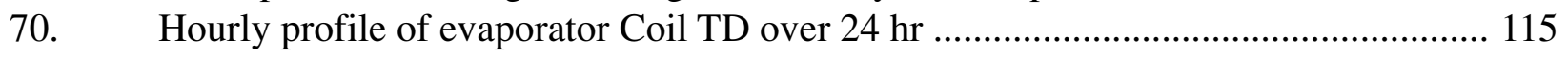

71. Hourly profile of evaporator coil superheat and total system subcooling

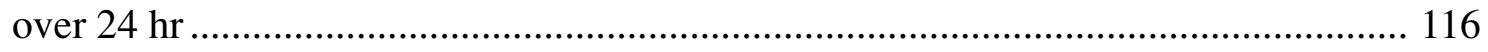

72. Hourly profile of ASH, lighting, and evaporator fan motor power over $24 \mathrm{hr} . . . \ldots \ldots . . . .116$

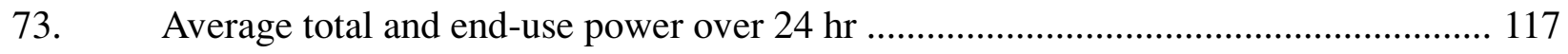

74. 2 min profile of product temperature at six different locations for top shelf

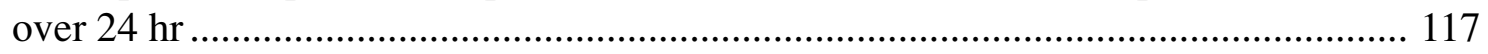

75. 2 min profile of product temperature at six different locations for second shelf

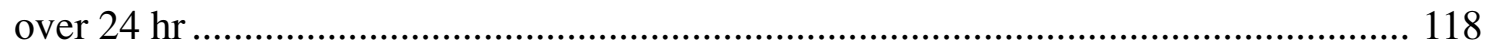

76. 2 min profile of product temperature at six different locations for third shelf

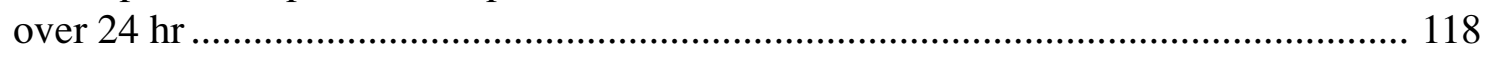

77. 2 min profile of product temperature at six different locations for bottom shelf

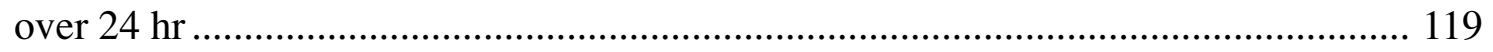

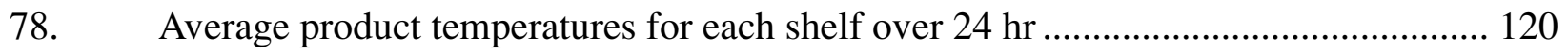

79. Average, coldest and warmest product temperatures over $24 \mathrm{hr}$.............................. 120

80. Infrared image of temperature gradient in front of the display case ........................ 121

81. Average air temperature at $2 \mathrm{ft}$ and $4 \mathrm{ft}$ away from the display case ........................ 122

82. Comparison of infiltration rate based on test results and CFD model....................... 123

83. Prototype display case showing fan and evaporator coil ....................................... 123

84. $2 \mathrm{~min}$ room dry-bulb and relative humidity data over $24 \mathrm{hr}$ period ........................ 124

85. $25 \mathrm{~min}$ suction and discharge pressures data over $24 \mathrm{hr}$ period .............................. 125

86. 2 min average discharge air temperature and product temperature data over $24 \mathrm{hr}$ period .......................................................................................... 125

87. 2 min mass of collected condensate data over $24 \mathrm{hr}$ period ................................. 126 
88. 2 min refrigeration effect and refrigeration mass flow rate data

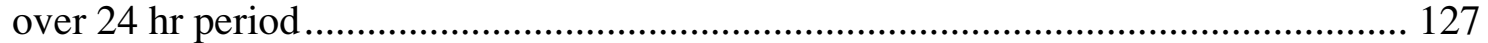

89. Average end-use power data over $24 \mathrm{hr}$ period ..................................................... 129

90. Compressor EER values for ARI 1200 energy consumption calculation ...................... 133

91. Results of CDEC calculation for the baseline and candidate display cases ................. 135 


\section{TABLES}

Table

Page

1. Major storage applications for refrigerated display cases ...................................... 8

2. Industry ranking of display case improvement technologies .................................... 22

3. Overall ranking of display case improvement technologies ..................................... 23

4. Results of industry survey of test display case selection ......................................... 24

5. Summary of responses from the Display Case Survey, Food Marketing

Institute Energy Conference, Cleveland, OH, September 9 to 12, 2001 ..................... 25

6. Agenda for the high-efficiency supermarket display case project industry

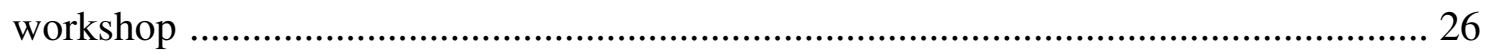

7. Comparison of test and manufacturer's published data for the test multi-deck

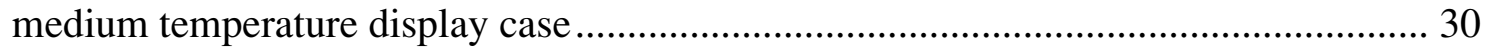

8. Configuration and storage categories for the case rating procedure ......................... 31

9. Proposed metrics for the draft display case efficiency rating procedure ..................... 31

10. Attendee list for the advanced supermarket display case workshop,

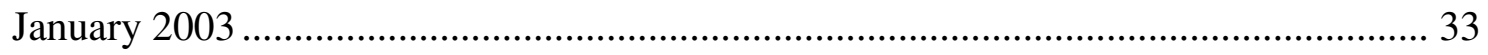

11. Attendees list for the advanced supermarket display case workshop, January 2004 ............................................................................................. 38

12. Summary of baseline and prototype case evaporator specifications ......................... 41

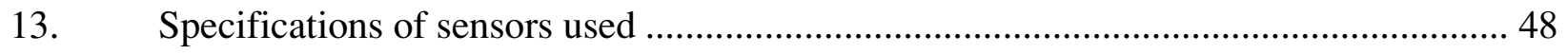

14. Comparative summary of test data and manufacturer's published data .................... 122

15. Comparison of key performance attributes of baseline and prototype cases ............. 129

16. Description of display case applications ............................................................... 131

17. Descriptions of available multi-deck meat/deli display cases .................................. 134

18. Candidate display cases that can be compared to the baseline ................................. 134

19. Summary of supermarket refrigeration energy savings estimates from use of advanced display cases (from DOE-2.3 simulations) 


\section{ACKNOWLEDGMENTS}

This report is an account of research sponsored by the U.S. Department of Energy, Office of Building Technology, State and Community Programs under contract number DE-AC0500OR22725 with UT-Battelle LLC.

The authors would like to thank Arun Vohra of the US DOE for his continued support and interest in this project.

The authors would also like to thank the many members of the supermarket refrigeration industry for their efforts on this project, including attending the project workshops and providing much helpful feedback on the project plans and results. Special thanks go to Massoud Neshan of Wolverine Tubing and Clark Bullard of the University of Illinois for their help in designing and fabricating the test evaporator coils.

We would like to recognize the outstanding work of several individuals who performed much of the laboratory work on this project:

Homayun Navaz - Kettering University.

Bruce Coburn - Southern California Edison RTTC.

Rafik Sarahadian - Southern California Edison RTTC. 


$\begin{array}{ll}\text { CFD } & \text { Computational Fluid Dynamics } \\ \text { CFM } & \text { Cubic Feet per Minute } \\ \text { DAG } & \text { Discharge Air Grill } \\ \text { DAT } & \text { Discharge Air Temperature } \\ \text { DAV } & \text { Discharge Air Velocity } \\ \text { DB } & \text { Dry Bulb } \\ \text { DOE } & \text { Department of Energy } \\ \text { DP } & \text { Dew Point } \\ \text { DPIV } & \text { Digital Particle Image Velocimetry } \\ \text { EB } & \text { Electronic Ballast } \\ \text { EEM } & \text { Energy Efficient Measures } \\ \text { ECM } & \text { Electronically Commuted Motor } \\ \text { IR } & \text { Infrared } \\ \text { LDV } & \text { Laser Doppler Velocimetry } \\ \text { LSHX } & \text { Liquid-to-Suction Heat Exchanger } \\ \text { NIST } & \text { National Institute of Standards and Technology } \\ \text { ORNL } & \text { Oakridge National Laboratory } \\ & \\ \text { Re } & \text { Reynolds Number based on the DAG width, } \frac{\bar{V} w}{v} \\ \text { RH } & \text { Relative Humidity } \\ \text { rpm } & \text { Revolutions per minute } \\ \text { RTD } & \text { Resistance Temperature Device } \\ \text { RAG } & \text { Return Air Grill } \\ \text { RTTC } & \text { Refrigeration and Thermal Test Center } \\ \text { SCE } & \text { Southern California Edison } \\ \text { SCT } & \text { Saturated Condensing Temperature } \\ \text { TXV } & \text { Thermostatic Expansion Valve } \\ \text { WB } & \text { Wet Bulb } \\ & \end{array}$


xviii 


\section{EXECUTIVE SUMMARY}

Supermarkets represent one of the largest energy-intensive building groups in the commercial sector, consuming 2 to 3 million $\mathrm{kWh} / \mathrm{yr}$ per store (ES-1). Over half of this energy use is for the refrigeration of food display cases and storage coolers. Display cases are used throughout a supermarket for the merchandising of perishable food products. The cases are maintained at air temperatures ranging from -10 to $35^{\circ} \mathrm{F}$, depending upon the type of product stored. The operating characteristics and energy requirements of the refrigeration system are directly related to the refrigeration load. The sources of the display case refrigeration load consist of:

- Moist and warm air infiltration through the open front of the case - air curtains are employed to inhibit this infiltration, but some ambient air is entrained, which adds a substantial portion to the refrigeration load.

- Heat conduction through case panels and walls.

- Thermal radiation from the ambient to the product and display case interior.

- Internal thermal loads - the use of lights, evaporator fans, periodic defrosts, and antisweat heaters adds to the refrigeration load of the display case as well as directly consuming electric energy.

The impact of each of these elements on the refrigeration load is very dependent upon case type (Figure ES-1). For example, air infiltration is the most significant portion of the refrigeration load for open, multi-deck cases, while radiation is the largest part of the load for tub-type cases. The door anti-sweat heaters represent a major share of the refrigeration load for frozen food door reach-in cases.

Figure ES-2 shows the distribution of display cases in a typical supermarket (ES-2). Open, multi-deck, medium temperature display cases typically comprise about half of the refrigerated fixtures in a store (ES-3). In addition, medium temperature fixtures and storage coolers account for roughly 70 to 75 percent of the total store refrigeration load with open, multi-deck cases contributing about $3 / 4$ of that fraction. Consequently, the focus of this investigation has tilted toward the open, vertical, multi-deck medium temperature case type.

Various technologies and control methods are energy efficiency measures (EEMs) that could be applied to display cases and result in the reduction of the refrigeration load and of the energy consumption of the supermarket refrigeration system. An extensive evaluation of the EEMs was conducted in order to select those that met the following criteria: 


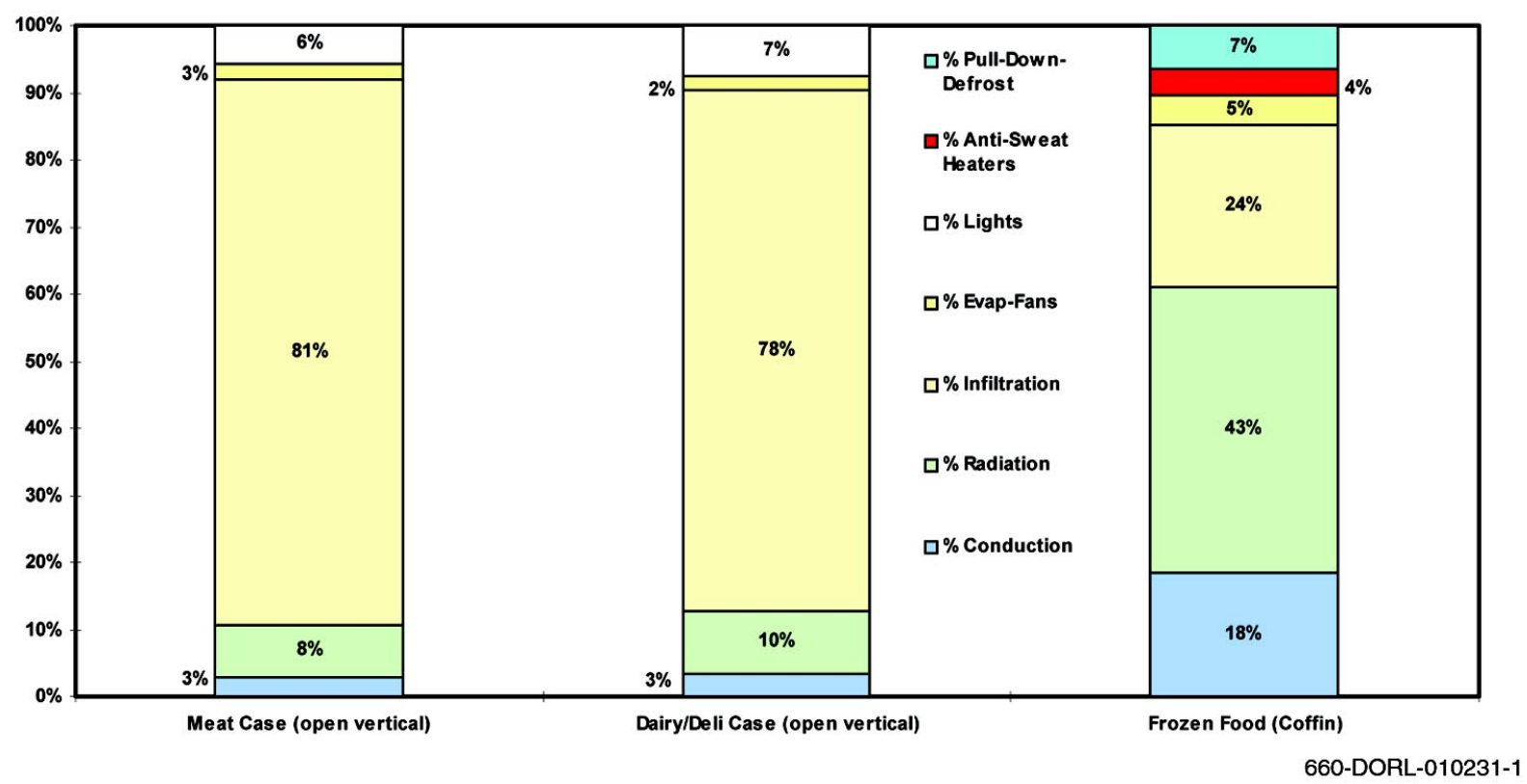

Figure ES-1. Refrigeration load percentage breakdown for meat, dairy/deli and frozen food fixtures (ES-2)

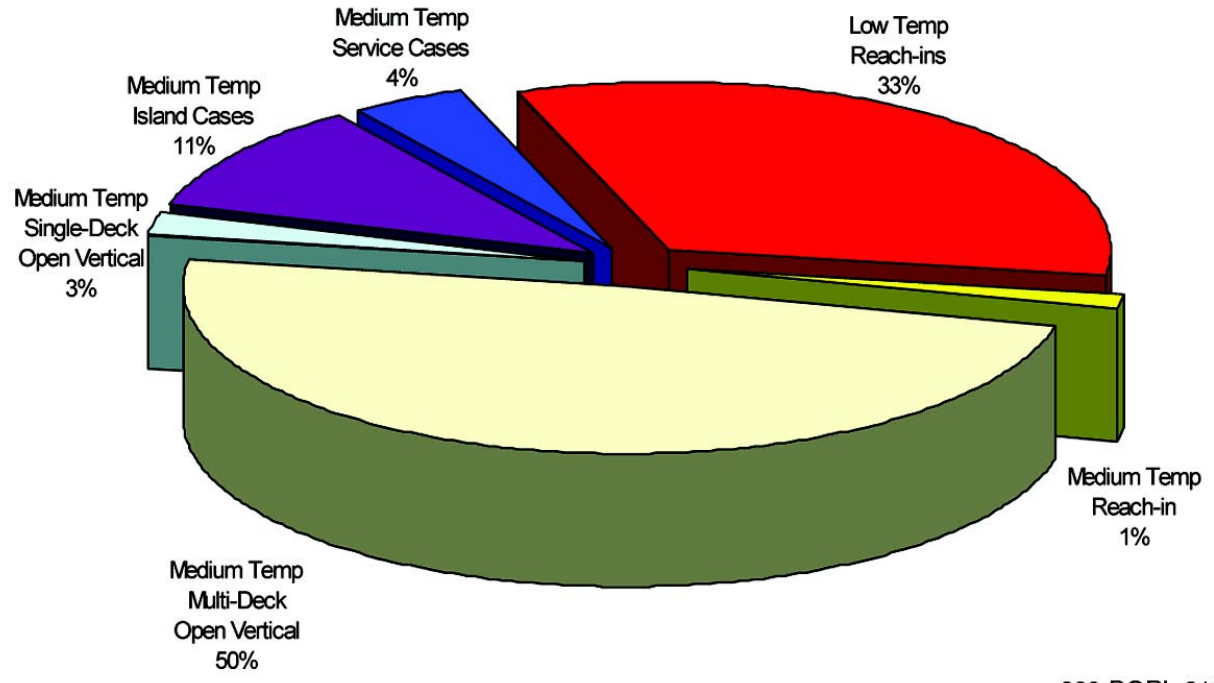

660-DORL-010231-2

Figure ES-2. Percentage breakdown of fixture lineal feet by type in a typical supermarket 
1. Near-term implementation - All EEMs considered could be implemented with existing refrigeration hardware and technology.

2. Potential for energy-efficiency improvements - Energy savings and/or refrigeration load reduction must be obtained by the implementation of the EEM.

3. Enhancement of the ability to maintain target product temperature - Proper operation of the display case and maintenance of the stored product temperature could not be compromised by the use of the EEM.

The energy impact of a number of viable display case EEMs was quantified by performing whole building hourly simulations. A special version of the U.S. Department of Energy's (DOE-2.3) program was used to develop a model of a supermarket. The model was then calibrated using available end-use monitored data to increase confidence in simulation results.

The following EEMs were simulated and their impact on the refrigeration or cooling load and energy usage of the facility were analyzed based on Los Angeles weather data.

1. Modular coil and defrost.

2. High efficiency evaporator, LSHX and advanced controls.

3. ECM for evaporator fan.

4. Tangential fan with ECM motor and VSD controller.

5. Improved air curtain.

6. Glass doors on open vertical medium temperature cases.

7. T8EB with enhanced interior film.

8. Combination of $1,2,3,4,5,7$.

9. Combination of $1,2,3,4,6,7$.

Model findings indicated that measures that impacted the infiltration to the open vertical medium temperature cases resulted in the lowest cooling loads. Combining the effects of these measures cooling energy of the site can be lowered from 2,676 kBtu/hr to either 2,259 for combination 8 or $1,882 \mathrm{kBtu} / \mathrm{hr}$ for combination 9 (16 percent to 30 percent). Implementation of EEM combination 8 is estimated to lower the annual refrigeration energy usage of the site by about 14 percent.

A standard medium temperature, open vertical, 4-deck display case that was $8 \mathrm{ft}$ in length was selected and evaluated for thermal performance benchmarking purposes. The baseline display case was equipped with three shaded pole fan motors, and two rows of standard T8 canopy lights with electronic ballast. Off-cycle defrost was initiated three times per day and terminated at coil outlet temperature of approximately $47^{\circ} \mathrm{F}$.

A combination of analytical and experimental methods was used to study the air curtain performance of the baseline case. These methods involved the use of digital particle image velocimetry (DPIV) and laser Doppler velocimetry (LDV) to visualize and quantify the air flow field along the plane of air curtain. The DPIV and LDV results were also used to calibrate a 
CFD model, which was later used to simulate the air curtain performance. Results obtained from CFD modeling were used to determine a $1^{\text {st }}$ generation redesign of the DAG configuration and velocity profile, which was aimed at minimizing the infiltration load of the display case.

Based on the findings from the previous work, a new display case design was developed and a prototype of this design was fabricated. Hill Phoenix built the shell of the prototype display case based on the new specification. The Southern California Edison's Refrigeration and Thermal Test Center (SCE RTTC) in conjunction with its partners including Wolverine, Sporlan Valve, General Electric, and University of Illinois designed, procured, and, in some instances, fabricated the following technologies:

1. Tangential evaporator fans.

2. Double-shafted electronically commuted evaporator motor with variable speed control.

3. Staggered fin design evaporator with enhanced tubing.

4. Micro-channel liquid-to-suction heat exchanger.

5. First generation redesigned air curtaingrill.

6. Dual-port thermostatic expansion valve.

The test setup and procedure for the baseline and prototype display cases followed guidelines specified in ASHRAE Standard 72-98. All tests were performed under steady-state conditions. The average discharge air temperature (DAT), which was the critical control point, was maintained at $30^{\circ} \mathrm{F}\left( \pm 0.5^{\circ} \mathrm{F}\right)$ to prevent maximum product temperature from exceeding $41^{\circ} \mathrm{F}$. The controlled environment chamber was maintained at a constant dry bulb (DB) temperature of $75.2^{\circ} \mathrm{F} \pm 2^{\circ} \mathrm{F}$ and wet bulb of $64.4^{\circ} \mathrm{F} \pm 2^{\circ} \mathrm{F}$, corresponding to 55 percent relative humidity (RH), throughout the entire $24 \mathrm{hr}$ test period. The entering liquid refrigerant temperature and pressure were maintained at $80^{\circ} \mathrm{F}$ and 214 psig (corresponding to SCT of $\sim 94^{\circ} \mathrm{F}$ ).

Table ES-1 gives a summary comparison between the test results of the baseline and prototype display cases. The prototype unit required roughly 20 percent less fan and lighting power than the baseline unit. Furthermore, the increase in refrigeration effect and decrease in refrigerant mass flow resulted in a drop of 10 percent in the compressor power requirement of the prototype case. Combining the savings obtained from the compressor, evaporator fan motor and lighting yielded 11 percent reduction in total power consumption. The energy-efficiency ratio (EER), which is defined as a ratio of total cooling load (Btu/hr) to sum of compressor, lights and fan motor power (watts), was increased by 22 percent for the prototype case. Additionally, the decrease in compressor power run time of the prototype case translated into a saving of 14 percent in compressor energy usage.

The cooling capacity was $12,989 \mathrm{Btu} / \mathrm{hr}$ and $12,977 \mathrm{Btu} / \mathrm{hr}$ for the baseline and the prototype, respectively. The prototype case experienced a 2 percent higher infiltration load than the baseline (10,835 versus $10,674 \mathrm{Btu} / \mathrm{hr})$. The increase in infiltration load of the prototype case can be attributed to modifications made to DAG configuration. The contribution of internal loads, however, decreased from 6 percent in the baseline case to 5 percent in the prototype. This reduction was due to less heat dissipation from the high-efficiency lighting system and the evaporator fan motor. 
Table ES-1. Comparison of test results for the baseline and prototype display cases

\begin{tabular}{|c|c|c|c|}
\hline & Baseline & Prototype & Difference \\
\hline SET $\left({ }^{\circ} \mathrm{F}\right)$ & 17 & 23 & $+6^{\circ} \mathrm{F}$ \\
\hline UA (Btu/hr- $\left.{ }^{\circ} \mathrm{F}\right)$ & 934 & 1,567 & $-68 \%$ \\
\hline Superheat $\left({ }^{\circ} \mathrm{F}\right)$ & 10.0 & 5.6 & $-4.4^{\circ} \mathrm{F}$ \\
\hline Subcooling $\left({ }^{\circ} \mathrm{F}\right)$ & 17.2 & 51.6 & $+34.4^{\circ} \mathrm{F}$ \\
\hline Refrigeration effect (Btu/b) & 53.6 & 63.5 & $-19 \%$ \\
\hline Refrigerant Mass Flow (lb/min) & 3.75 & 3.41 & $9 \%$ \\
\hline Total Refrigeration Load (Btu/hr) & 12,989 & 12,977 & - \\
\hline Conduction Load (Btu/hr) & 526 & 514 & $2 \%$ \\
\hline Radiation Load (Btu/hr) & 1,016 & 1,014 & $0.2 \%$ \\
\hline Internal (lights and fans) Load (Btu/hr) & 773 & 614 & $21 \%$ \\
\hline Infiltration Load (Btu/hr) & 10,674 & 10,835 & $-2 \%$ \\
\hline Mass of Condensate Collected over $24 \mathrm{hr}(\mathrm{lb})$ & 83.30 & 86.65 & $-4 \%$ \\
\hline Compressor Power (W) & 1,460 & 1,315 & $10 \%$ \\
\hline Compressor Energy (kWh) & 32.6 & 27.9 & $14 \%$ \\
\hline Fan Motor (W) & 115 & 92 & $20 \%$ \\
\hline Lighting (W) & 112 & 88 & $21 \%$ \\
\hline Total System Power (W) & 1,687 & 1,495 & $11 \%$ \\
\hline Total System Energy (kWh) & 38.0 & 32.2 & $15 \%$ \\
\hline EER $(\mathrm{Btu} / \mathrm{hr} / \mathrm{W})$ & 7.12 & 8.65 & $-22 \%$ \\
\hline Maximum Product Temperature $\left({ }^{\circ} \mathrm{F}\right)$ & 39.4 & 39.6 & $+0.2^{\circ} \mathrm{F}$ \\
\hline
\end{tabular}

In summary, the prototype system energy usage improved while maintaining target product temperatures. The energy savings achieved by the prototype display case was $6 \mathrm{kWh} /$ day, which was 15 percent of the daily energy consumption of the baseline display case.

Despite initial attempts to redesign the DAG to effect reductions in air infiltration, the infiltration load of the prototype display case remained almost the same as that of the baseline. Further research is highly recommended to improve our understanding of air curtain performance and develop more effective means to reduce infiltration loads on open, vertical display cases.

\section{References}

ES-1. Field Testing of High-Efficiency Supermarket Refrigeration, Foster-Miller, Inc., EPRI report no. TR-100351, Electric Power Research Institute, Palo Alto, CA, December 1992.

ES-2. Faramarzi, R. 1999. Efficient display case refrigeration. ASHRAE Journal (November): 46.

ES-3. A.D. Little, Energy savings potential or commercial refrigeration equipment, prepared for Office of Building Technologies, U.S. Department of Energy. Cambridge, MA, June 1996. 


\section{INTRODUCTION}

Supermarkets represent one of the largest energy-intensive building groups in the commercial sector, consuming 2 to 3 million $\mathrm{kWh} / \mathrm{yr}$ per store. Several studies have shown that annual electricity consumption ranges from 1 to 1.5 million $\mathrm{kWh} / \mathrm{yr} / \mathrm{store}$ for refrigeration $(\underline{1,2})$. Recent field tests have tended to confirm that this figure is still a good estimate. Data from a field test in a 50,000 $\mathrm{ft}^{2}$ store in Massachusetts indicate that a full year's energy use (for compressor racks and condensers only) would be about 925,000 to $950,000 \mathrm{kWh}(\underline{3})$. Data for a similar size store in southern California indicate annual usage of about $1,500,000 \mathrm{kWh}$ for all refrigeration including case lights, fans, heaters, etc. (see subsection 7.5 in this report).

A major factor affecting design and operation of the refrigeration system is the cooling load requirements of the display cases. This refrigeration load determines the amount and size of the compressors and condensers that must be installed and greatly influences the energy consumption for refrigeration. Display cases are used throughout a supermarket for the merchandising of perishable food products. The cases are maintained at air temperatures ranging from -25 to $35^{\circ} \mathrm{F}$, depending upon the type of product stored. The necessary cooling for these cases is provided by a large vapor compression refrigeration system. The operating characteristics and energy requirements of the refrigeration system are directly related to the refrigeration load associated with maintaining display case temperature. The sources of the display case refrigeration load consist of:

- Moist and warm air infiltration through the open front of the case - air curtains are employed to inhibit this infiltration, but some ambient air is entrained, which adds a substantial portion to the refrigeration load.

- Heat conduction through case panels and walls.

- Thermal radiation from the ambient to the product and display case interior.

- Internal thermal loads - the use of lights, evaporator fans, periodic defrosts, and antisweat heaters adds to the refrigeration load of the display case as well as directly consuming electric energy.

The impact of each of these elements on the refrigeration load is very dependent upon case type. For example, air infiltration is the most significant portion of the refrigeration load for open, multi-deck cases, while radiation is the largest part of the load for tub-type cases. Based on independent laboratory test results (4), infiltration accounts for roughly 80 percent and 
40 percent of the cooling load of open vertical and tub cases, respectively. The door anti-sweat heaters represent a major share of the refrigeration load for frozen food door reach-in cases.

Various control methods and technologies exist that are energy efficiency measures (EEMs) that could be applied to display cases and result in the reduction of the refrigeration load and of the energy consumption of the refrigeration system. Examples of such technologies include:

- Energy-efficient display case fans and lights - The use of more efficient fan motors and lights can reduce both refrigeration load and direct energy consumption.

- Controls for anti-sweat heaters also help in load and energy reduction.

- Higher R-factor insulation - The use of higher R-factor insulation, such as vacuum panels helps cut conduction heating of the cases.

- Improved air curtain design - Effective and optimized design of air curtains can be achieved and implemented, which will lead to reduced infiltration of ambient air and humidity into the display case.

- More efficient coil design - The evaporator coil can be designed to operate at a close temperature difference (TD) so that the evaporator temperature can be raised for a given desired case temperature. More efficient coil design where evaporation occurs over the greatest length of coil tubing helps to maintain a reasonable evaporator size while also improving the uniformity of food temperature.

- Electronic expansion valves - Allows tighter control of superheat so that coil is used as efficiently as possible.

- Special Polymer doors for open vertical and reach-in cases that will eliminate the need for heating glass.

- Frost tolerant surfaces and better evaporator fan design - These features would result in less need for coil defrost, which will decrease the "pull-down" requirement of the refrigeration load.

- Demand defrost controls - Triggers defrost only when needed, reducing the number of defrosts and the added load associated with returning the case to the desired temperature (pull-down load).

- High-efficiency liquid-to-suction heat exchanger - Provides subcooling of liquid refrigerant through useful superheat and allows the evaporator coil to operate with low superheat at the evaporator outlet.

Energy savings gained through the use of EEMs in refrigerated display cases could be on the order of 20 percent of total refrigeration energy use. 
A possible method to accelerate the implementation of the EEMs is the development of an efficiency rating for display cases, which can be made by comparing the test results for the display case with that of a baseline of similar design. Standardized display case testing methods such as ASHRAE Standards 72 and 117, used throughout the industry, exist for the measurement of display case refrigeration loads $(\underline{5}, \underline{6})$. A methodology to evaluate display case performance is also available through the ARI Standard 1200 (ㄱ). These methods could be readily adapted to a rating system to determine energy efficiency. Display cases showing adequate improvement through a combination of refrigeration load reduction and in-case energy use, while meeting design discharge air and stored product temperature requirements will be considered highly efficient and will receive an "Energy-Efficient" label. By developing simple labeling mechanisms for refrigerated display cases, supermarket and grocery operators will easily be able to identify money saving and environmental fixtures. The lack of clear, accurate, and unbiased information about energy-efficient display cases, coupled with the great emphasis of market factors on the first cost, has long been a barrier to the successful implementation of these products in the marketplace. The Energy-Efficient label can make it easy for supermarket operators to choose those display cases that will reduce their energy cost without sacrificing performance. Since the display cases are very visible in the supermarket sales area, efficiency labeling is expected to be readily noticed by consumers; this can be a highly desirable feature for both supermarket operators and display case manufacturers. Additional environmental benefits can be obtained including reduction of refrigerant leakage and lower production of greenhouse gases.

Recognizing the importance of energy-efficiency in commercial refrigeration, the U.S. DOE, through Oak Ridge National Laboratory, initiated a research project to investigate the potential for energy savings through the implementation of EEMs in display cases. The major tasks of the project consisted of:

- $\quad$ Survey existing display cases - Performance data were gathered for existing supermarket display case manufacturers by common display case types and by food-storage application. These data can be used to determine the baseline performance for each case type and application, which can be used as a basis of comparison for performance rating.

- Determine energy enhancements and technologies available - An investigation was undertaken to determine components, designs, and control methods that are near-term or commercially available to enhance the performance of display cases by lowering refrigeration load and reducing energy consumption.

- Analyze display case performance with EEMs - Estimates of the display case performance were made when EEMs were implemented. Both refrigeration load and energy reductions were considered.

- Determine energy and economic savings to supermarkets - Annual energy analysis of supermarkets was conducted to determine the energy savings and economic benefits obtained by the implementation of high-efficiency display cases. All EEMs selected 
were evaluated in this fashion prior to implementation in case testing. Annual energy savings were estimated for 6 geographic locations in the United States.

- Experimentally investigate display case air curtains - Advanced air velocimetry techniques, coupled with computational fluid dynamics (CFD) modeling, were used to characterize the air curtain of an open, 4-deck, display case and to identify methods to reduce the infiltration of ambient air into the case.

- Incorporate selected EEMs in a test display case - An open, 4-deck, medium temperature display case was obtained for testing. Refrigeration performance of the case in its baseline configuration was thoroughly measured and documented. The case was then modified and equipped with selected EEMs which were:

- Low TD evaporator equipped with a dual-port thermostatic expansion valve (TXV).

- Tangential fans located at the inlet of the evaporator and equipped with an electronically commutated motor (ECM). The fan and motor were arranged for variable-speed operation; however, this feature was used only to vary airflow as part of the air curtain investigation.

- High-efficiency liquid-suction heat exchanger, that used micro-channel geometry to insure full subcooling of the refrigerant.

- High-efficiency lighting, consisting of T8 fluorescent bulbs and electronic ballasts that were mounted external to the display case.

- A $1^{\text {st }}$ generation redesign of the air curtain discharge air grill (DAG) based on the measurements and CFD modeling mentioned above.

Performance testing was then conducted to allow comparison of the prototype case with the baseline.

- Develop energy efficiency rating procedures for display cases - A draft methodology was prepared that will allow "energy-efficient" cases to be identified and marketed as such.

- Involve the supermarket industry - Regular workshops were scheduled throughout the project so that industry representatives could review results of the project and provide input. Three such workshops were held, and several industry surveys related to the project were conducted. 


\section{SUPERMARKET REFRIGERATION AND DISPLAY CASE OPERATION}

A representative supermarket layout is shown in Figure 1. Refrigerated fixtures are located throughout the store, because of the large amount of perishable food products that are sold. These fixtures fall into 2 categories, which are display cases and walk-in storage coolers. The display cases are located on the sales floor and are designed to refrigerate food products while providing a place to merchandise them. Walk-in coolers are used to store food products during the time period between receiving the product and placing the product out for sale.

Refrigeration of the display cases and walk-in coolers is done through the use of direct expansion refrigerant/air coils located in each case or cooler. Refrigerant piping is provided to each coil to supply liquid refrigerant to the coil and remove evaporated refrigerant from the coil and return the gas to the refrigeration compressors.

The compressors are located in a machine room in a remote part of the store, such as in the back room area or on the roof. The system condensers are located either in the machine room, or more likely, on the roof above the machine room.

The purpose of refrigerated display cases in a supermarket is to provide temporary storage for perishable foods prior to sale. Most of the design characteristics and general shape and layout of display cases are based on marketing specifications and constraints. The configuration of display cases falls into essentially four different categories, which are (Figure 2):

- Tub: The tub case is often used for the storage and display of frozen foods and meats. Tub cases operate at a very uniform temperature and require the least amount of refrigeration per foot of any display case type. The primary disadvantage of the tub is a low product storage volume per square foot of sales area taken up by the case.

- Open-front multi-deck: This case type possesses the largest storage volume per square foot of floor area, because of the use of an upright cabinet and shelves. Refrigeration requirements are very high for multi-deck cases, including a large latent load portion due to the entraining of ambient air in the air curtain passing over the opening of the case.

- Glass door reach-in: The reach-in case has glass doors over the opening of the case; these must be opened for product removal and stocking. Reach-in cases are used in supermarkets primarily for frozen foods, because of their ability to contain the cold refrigerated air, which reduces the "cold aisle" problem. The refrigeration loads associated with the glass door reach-in case are normally less than those for the multi- 


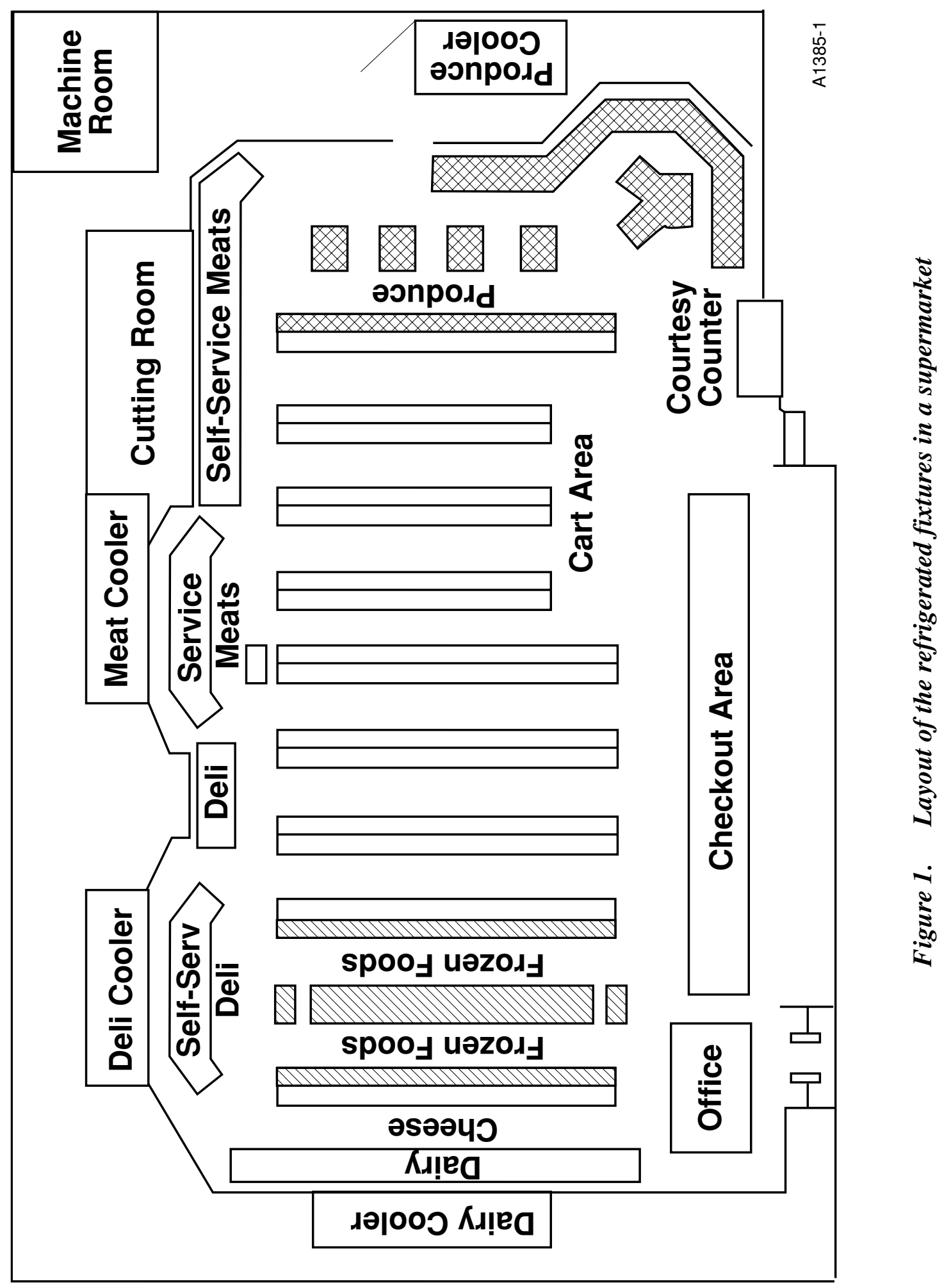



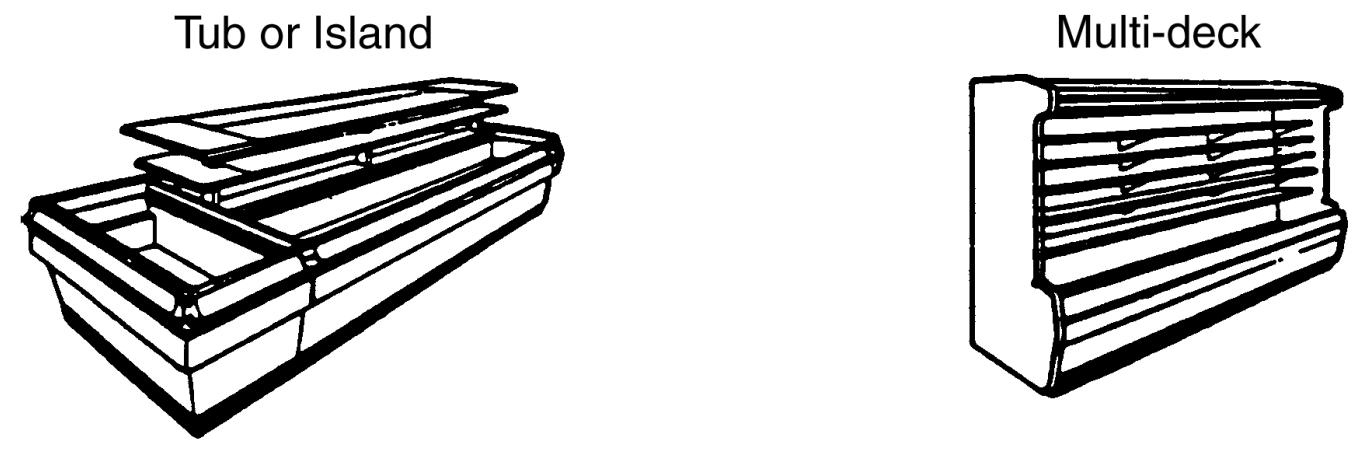

Glass Door Reach-in
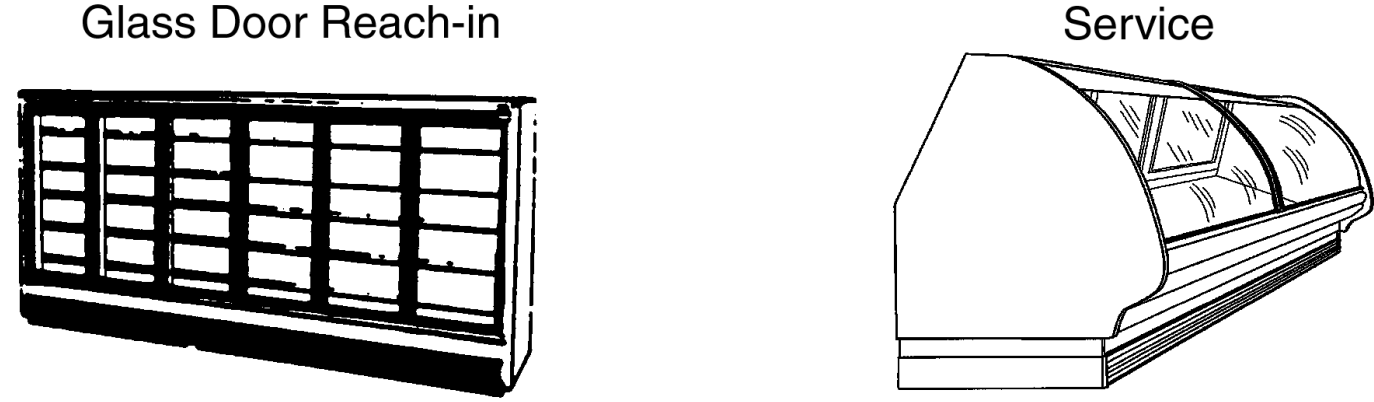

599-DORL-010231-1

Figure 2. Display case types employed in supermarkets

deck but greater than for the tub case. Glass door cases are, however, equipped with antisweat electric heaters in the doors to prevent fogging and decreased visibility of the product.

- Single-deck or service: Open single-deck cases are commonly used for display of fresh meat products. The service display case is a single-deck case equipped with sliding doors in the back for access by serving people and a glass front to show product to customers. Cases of this type are commonly seen in the deli and meat departments of supermarkets.

Display cases have been developed and refined for specific merchandising applications, and cases of each type listed above exist specifically for the storage and display of specific food types. Table 1 lists the major food storage applications for which display cases are provided. Each storage application also has a specific food storage temperature requirement. The control of the food storage temperature is provided by the temperature of the air leaving the display case evaporator (referred to as the discharge air temperature). Table 1 gives the typical operating range for discharge air temperatures for the major food storage applications.

Figure 3 depicts the operation of a typical open vertical display case. Refrigeration of the product stored in the case is provided by the circulation of cold air around the case perimeter and through the back wall of the case. The display case walls are normally contain either fiberglass or foam insulation to minimize the heat conducted and also prevent condensation from forming on the outer surface. For open display case types, the air passes across the discharge air grill 
Table 1. Major storage applications for refrigerated display cases

\begin{tabular}{|l|c|}
\hline Storage Application & $\begin{array}{c}\text { Discharge Air Temperature } \\
\left({ }^{\circ} \mathrm{F}\right)\end{array}$ \\
\hline Dairy & 30 to 34 \\
\hline Deli & 31 to 34 \\
\hline Meat/Seafood & 27 to 29 \\
\hline Produce & 31 to 34 \\
\hline Beverage & 36 \\
\hline Frozen Food & -15 to -2 \\
\hline Ice Cream & -25 to -12 \\
\hline
\end{tabular}

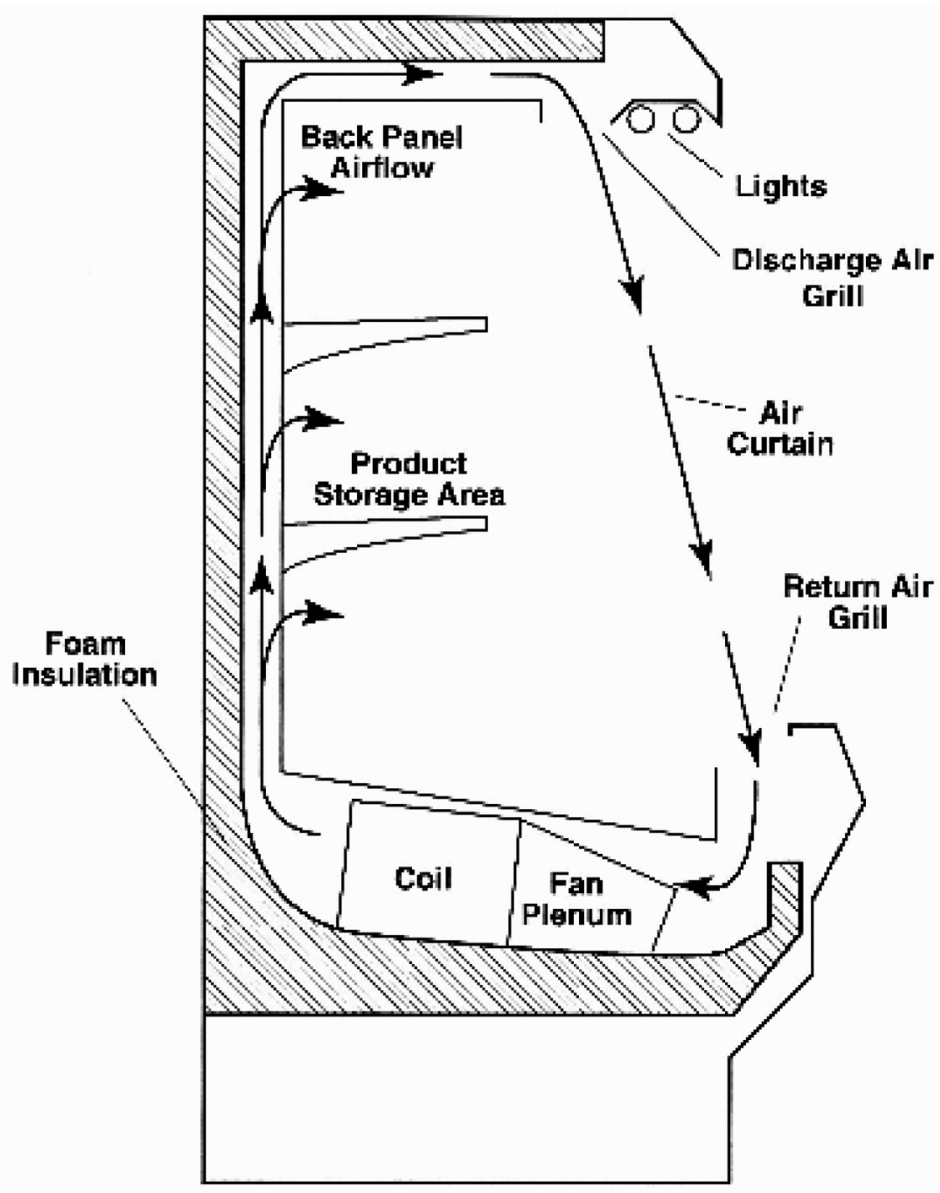

660-DORL-010231-3

\section{Figure 3. Typical open, vertical multi-deck display case air circulation pattern}

opening to form an air curtain that creates a barrier between the cold air in the display case and the ambient air in the store. The airflow is returned through the return air grill to the evaporator for cooling. The evaporator contains refrigerant that is first passed through an expansion valve and then through multiple tube passes that are in contact with the circulating air. Heat transferred from the air evaporates the refrigerant, which in turn provides the desired cooling of the air. 
The refrigeration load of the case is the amount of heat energy that must be removed from the display case in order to maintain the product in the case at the desired storage temperature. The refrigeration load of a display case is most often given at a particular discharge air temperature, since this value is easier to measure (and control) than the temperature of the stored product. The standard rating condition to specify the refrigeration load of a display case is for operation in an indoor environment with a $75^{\circ} \mathrm{F}$ dry-bulb temperature and a relative humidity of 55 percent. The heat energy that makes up the refrigeration load comes from a number of sources, which can be categorized as follows:

- Conduction - Ambient heat that passes through the walls of the display case and is intercepted by the air flowing around the perimeter of the display case.

- Radiation - Thermal radiation heat transfer occurs between the interior of the display case and the surrounding ambient environment.

- Convection (air entrainment) - The air curtain passing across the opening of the display case will mix with and entrain part of the surrounding ambient air, which is then returned to the case evaporator. The heat load due to the entrained air consists of both sensible and latent heat. The latent heat load results in the sublimation of frost on the evaporator surface. Ambient air entrainment occurs in all display case types, but represents the largest portion of the refrigeration load for open, multi-deck cases.

- Internal - Heat energy is generated by the use of electric energy in the display case for:

- Lights - fluorescent light fixtures are installed in the display cases for illumination of the product. Heat from the ballasts may also enter the case if the ballast is installed in the refrigerated portion of the case.

- Fan Motors - the electric energy associated with the fans used to circulate air around the display case.

- Anti-Sweat Heaters - are installed in glass doors and on other surfaces that operate at a temperature below the ambient dew-point temperature. If heaters are not installed, condensation will form on these surfaces.

The contribution of each load source will vary according to display case type. The refrigeration load of open multi-deck display cases is dominated by air entrainment. Internal electric loads represent a significant portion of the refrigeration load of reach-in frozen food cases. For single-deck and tub cases, radiation heat transfer accounts for a large fraction of the refrigeration load (ㅁ).

The frost formation that is seen on evaporator surfaces is another important factor in the operation of display cases. Without periodic removal, the frost will accumulate and eventually block the airflow passages of the evaporator, resulting in loss of cooling capacity. The practice in supermarket refrigeration systems is to provide defrost to the display cases on a scheduled basis. Several different methods are employed for defrost, which are: 
- Off-Cycle - Refrigeration to the case is shut off and the evaporator is allowed to warm above the melting temperature of the frost. This method is commonly used for display cases operating at the highest discharge air temperatures ( 34 to $\left.37^{\circ} \mathrm{F}\right)$, because frost loading is relatively light. Off-cycle defrost is also used where the product is not sensitive to air temperature change, such as milk and other dairy products. For frozen food or meat, off-cycle defrost is not appropriate.

- Electric - Electric heaters are installed at the inlet of the evaporator so that the circulated air can be heated. The warm air passes through the evaporator where it provides the heat needed to melt the frost. Electric defrost is used in all applications and is considered the most reliable method of defrost, but is also the most energy consuming.

- Hot Gas - Discharge refrigerant gas is piped from the compressor rack to the display where the refrigerant is condensed by melting the frost. The piping is arranged so that the liquid refrigerant is returned to the compressor rack for distribution to other display cases in the system. Hot gas defrost can remove the frost fastest of all the defrost methods and tends to have the least impact on case air and product temperatures. Hot gas is the most costly defrost method to implement because of the extensive piping and controls needed.

Defrost is significant to the operation of the display case, because of the air and product temperature rises seen during defrost. If not done correctly, the product can be damaged. The display case must be returned to temperature after defrost is performed, which means a large transient (or "pull-down") refrigeration load is imposed on the refrigeration system. In some instances, the pull-down of the defrosted display case can influence refrigeration to other display cases as well.

The number of defrosts required for a display case is dependent upon its type. Open, multideck display cases will require several, while tub and reach-in cases normally have only one defrost per day.

Defrost scheduling is normally controlled by a time clock that initiates defrosts for each case at specific times each day.

As mentioned previously, electric energy is used directly in display cases for air circulation fans, lighting, and for anti-sweat heaters. The standard motors employed for the air circulation fans are shaded-pole (SP) with efficiencies of the order of 19 to 25 percent. More efficient motors are available, consisting of either permanent-split-capacitor (PSC) or electronically commutated (EC) that have efficiencies of about 35 to 45 percent ()ㅡ).

Display case lighting is normally fluorescent, employing type T-8 bulbs with the ballasts mounted in the electrical raceway of the display case. For open, multi-deck cases, standard lighting consists of 1 or 2 rows of fluorescent bulbs located in the top canopy. Additional lighting is sometimes placed along the lower rail of the case and on the bottom of each shelf to 
illuminate the shelf below it. Lighting in frozen food reach-in cases is located in the vertical posts between the doors. Single-deck, island, and tub cases normally have no lighting.

Anti-sweat heaters are used in display cases to prevent condensate formation on cold surfaces. Heaters are placed in the glass doors of frozen food reach-in cases to prevent condensation and fogging on the glass. These particular heaters can be substantial in size, on the order of $200 \mathrm{~W} /$ door. Door heater energy consumption can be significant for a large frozen food aisle in a supermarket, particularly if no anti-sweat heater controls are provided. The anti-sweat heater controls consist of a device capable of cycling the door heaters based on either doortemperature or ambient-dew-point measurement. Other display cases also employ anti-sweat heaters, normally along the outer edges of the case. These edge heaters are not as large as those found in the frozen food reach-in cases. 


\section{SURVEY OF PRESENT SUPERMARKET DISPLAY CASES}

A survey was conducted of presently manufactured supermarket display cases. Results of the survey will allow estimates to be made of present electric energy use for the operation of these cases either as direct consumption by the cases or by refrigeration compressors and condensers in order to provide refrigeration to these cases. All cases listed are remotely refrigerated. No selfcontained units are included.

The survey was conducted by obtaining technical data from the four leading display case manufacturers in the United States. These manufacturers are:

- Hussmann Corporation.

- Hill Phoenix.

- Tyler Refrigeration Corporation.

- Kysor Warren.

The display cases listed have been categorized into four categories, which are:

- Open Multi-Deck - Cases with multiple shelves, or decks, for product display. In general multi-deck cases have 2, 3, 4, or 5 decks. Some variations are noted as to the height of the front panel of the case.

- Open Single Deck - Cases with a single surface for product display with and without a glass front. This category includes service cases.

- $\quad T u b-$ Cases with a well-type product area.

- Glass Door Reach-in - Cases with glass door fronts.

The product display has been divided into the categories of dairy, deli, meat, produce, beverage, bakery, frozen food, and ice cream. In many instances, a particular case can be used for several of these products. Where the product displayed affects the operating temperatures or refrigeration loads, a separate entry for the case is provided for each product. If the specified temperatures and refrigeration loads are the same for multiple products, the products used in that case are noted in the description. 


\subsection{Survey Contents}

The following information is provided for each case:

- Case Description and Model Number - A general description is given along with the model number of the manufacturer.

- Evaporator and Discharge Air Temperatures - The evaporator and discharge air temperatures specified by the manufacturer for that particular case and product use are shown. Discharge air temperature values not available for some Tyler service cases.

- Refrigeration Load - The refrigeration load is specified as Btu/h/ft of case length for open cases and as Door/door for glass door reach-in cases. All refrigeration loads given are for use with parallel compressor systems. Refrigeration loads are determined by the manufacturers using the methods outlined in ASHRAE standards 72 and $117(\underline{5}, \underline{6})$. Refrigeration loads are not available for some Kysor-Warren service cases.

- Display Case Power - The power draw for case fans, lights, and anti-sweat heaters are listed.

The display case power values were determined from power values given in the manufacturer's literature. If wattages were not listed, they were calculated from electrical voltage and current values. The relation used to determine the wattage was

$$
\text { Power }(\mathrm{W})=\mathrm{VI} \cos \phi
$$

where

$$
\begin{array}{ll}
\mathrm{V} & =\text { Voltage }(115 \text { or } 120 \mathrm{VAC}) \\
\mathrm{I} & =\text { Currents }(\text { Amps }) \\
\cos \phi & =\text { power factor }
\end{array}
$$

The power factor values used were: 0.5 for fans, 0.9 for lights, and 1.0 for heaters. The fan wattage listed is for standard fans. Lighting consisted of that specified as standard by the manufacturer, and usually consisted of 1 row of lighting. Power values are given for a case length of $12 \mathrm{ft}$ for open cases, or 4-doors for reach-in cases. Specialty cases of lengths less than $12 \mathrm{ft}$ are noted. 


\subsection{Survey Results}

\subsubsection{Kysor-Warren}

\begin{tabular}{|c|c|c|c|c|c|c|c|c|}
\hline \multirow{2}{*}{ Display Case Type } & \multirow{2}{*}{ Application } & \multirow{2}{*}{$\begin{array}{l}\text { Manufacturer's } \\
\text { Model No. }\end{array}$} & \multicolumn{2}{|c|}{ TEMPERATURES $\left({ }^{\circ} \mathrm{F}\right)$} & \multirow{2}{*}{$\begin{array}{l}\text { Cooling Load } \\
\text { (Btu/hr-ft) }\end{array}$} & \multicolumn{3}{|c|}{ Case Power (Watts) } \\
\hline & & & Evaporator & Discharge Air & & Fans & Lights & Heaters \\
\hline \multirow[t]{17}{*}{ Open Muffi-Deck } & Dany, 5 deck standard height $\left(23^{3}\right)$ & D6 & 20. & 30 & 1300 & 20 & 138 & 0 \\
\hline & Dairy, 5 Deck, high front (28") & D6H & 20 & 30 & 1206 & 120 & 138 & 0 \\
\hline & Dairy, 5 Deck, low front $\left(19^{\prime}\right)$ & D6L & 20 & 30 & 1394 & 120 & 138 & 0 \\
\hline & Dairy, SDeck, Fear Low? & $06 \mathrm{R}$ & 20 & 25 & 43 & 100 & 138 & 0 \\
\hline & Dairy, 5 Deck, low front, Rear Load & D6RL & 20 & 28 & 1394 & 160 & 138 & \\
\hline & Deli, 5 Deck, standard front $\left(23^{\prime \prime}\right)$ & D6 & 15 & 26 & 1388 & 120 & 138 & 0 \\
\hline & Deli, 5 Deck, high front (27") & D6H & 15 & 26 & 1388 & 120 & 138 & 0 \\
\hline & Deli, 5 Deck, low front (19") & D6L & 15 & 26 & 1394 & 120 & 138 & 0 \\
\hline & Meat/Deli, 3 Deck, front glass & M3AG & 15 & 24 & 1000 & 144 & 85 & 148 \\
\hline & Meat/Deli, 4 Deck Narrow & MAN & 20 & 24 & 1430 & 60 & 85 & \\
\hline & Meat/Deli, 4 Deck & M4A & 15 & 24 & 1456 & 144 & 190 & 92 \\
\hline & Meat/Deli, 4 Deck, front glass & M4AG & 15 & 24 & 1404 & 144 & 190 & 92 \\
\hline & Meat/Deli, 5 Deck & M5 & 15 & 24 & 1643 & 144 & 190 & 52 \\
\hline & Meat/Deli, 5 Deck, front glass & MSG & 15 & 24 & 1643 & 144 & 190 & 152 \\
\hline & Frozen Food, 5 Deck, high front $\left(30^{\prime \prime}\right)$ & LSF & $-20)$ & -2 & 1325 & 357 & 435 & 989 \\
\hline & Frozen Food, 5 Deck, standard front $\left(26^{\prime \prime}\right)$ & L5 & -20 & -2 & 1508 & 357 & 435 & 989 \\
\hline & Ice Cream & 15 & 40 & -18 & & 357 & 435 & 989 \\
\hline \multirow[t]{22}{*}{ Open Single Deck } & Meat Del sing it Ded & MiA & 15 & 24 & $54 !$ & 72 & 0 & 140. \\
\hline & Meat/Deli, Single Deck, front glass & M1 AG & 15 & 24 & 515 & 72 & 0 & 197 \\
\hline & Service Deli, 25" Front & S35J J & 20 & 30 & & 59 & 135 & 0) \\
\hline & Service Deli, $29^{\prime \prime}$ Front & S39.J & 20 & 30 & & 59 & 135 & 0 \\
\hline & Service Meat, 25" Front & S35S & 20 & 30 & & 59 & 135 & o) \\
\hline & Service Meat, 29" Front & S39S & 20 & 30 & & 59 & 135 & 0 \\
\hline & Service Fish, 25" Front & S35SF & 20 & 36 & & 0 & 135 & o) \\
\hline & Service Fish, $29^{\prime \prime}$ Front & S39SF & 20 & 36 & & 0 & 135 & 0 \\
\hline & Service Deli Lift Front ( $9 \mathrm{ft}-10 \mathrm{in}$. length) & VRM-950 & 20 & 27 & 500 & 35 & 93 & 81 \\
\hline & Service Meat Lift Front ( $9 \mathrm{ft}-10$ in. length) & VRM-950 & 15 & 25 & 550 & 35 & 93 & 81 \\
\hline & Service Deli (9ft - 10 in length) & VRMB-950 & 15 & 27 & 687 & 69 & 93 & 80 \\
\hline & Service Meat \& Fish (9tt 10 in length) & VRH & 10) & 27 & 838 & 4) & 104 & 80 \\
\hline & Service Deli Open Front ( $9 \mathrm{ft}-10$ in. length) & VPA-KN & 10 & 25 & 1301 & 173 & 311 & 288 \\
\hline & Service Bakery (9f - 10in length) & VRB-750 & 20 & 28 & 687 & 347 & 207 & 81 \\
\hline & Self-Service Bakery & B33 & 20 & 32 & 1040 & 120 & 106 & 0 \\
\hline & Produce Bulk, High Back & HZV & 20 & 37 & 876 & 80 & 184 & 0 \\
\hline & Produce Precut, High Back & $\mathrm{H} 3 \mathrm{ZV}$ & 20 & 37 & 1068 & 60 & 184 & \\
\hline & Produce Bulk, Single Deck & $\mathbf{z v}$ & 20 & 37 & 442 & 40 & 0 & ) \\
\hline & Frozen Food, Single Deck wall & $\mathbf{X I}$ & -20 & -10 & $4(x)$ & 5) & 0 & 280 \\
\hline & Frozen Food, Single Deck wall front glass & XLG & -20 & -10 & 425 & 50 & 0 & 202 \\
\hline & Ice Cream, Single Deck wall & $\mathbf{x L}$ & -30 & -20 & 415 & 72 & 0 & 279 \\
\hline & Ice Cream, Single Deck wall front glass & XLG & -30 & -20 & 440 & 72 & 0 & 279 \\
\hline \multirow[t]{6}{*}{ Tub } & Cheese (wide island) & crw & 15 & 35 & 1018 & 58 & 0. & 0 \\
\hline & Produce Bulk, Island & EBZ & 20 & 37 & 850 & 80 & 184 & 0 \\
\hline & Frozen Food, Wide Is land & WTL & -20 & -10 & 749 & 180 & 0 & 336 \\
\hline & Frozen Food, Wide Island glass & WTLG & -20 & -10 & 796 & 180 & 0 & 462 \\
\hline & Ice Cream, Wide Island & WTL & -30 & -20 & 806 & 180 & 0 & 336 \\
\hline & Ice Cream, Wide Island glass & WTLG & -30 & -20 & 822 & 180 & 0 & 462 \\
\hline \multirow{4}{*}{ Gass Door Roach in } & & & & & (Btu/hr-dr) & & & \\
\hline & Dairy/Deli & DVs & 20 & 36 & 1360 & 83. & 467 & 218 \\
\hline & Frozen Food, Reach-in & LV5 & -15 & -2 & 1560 & 83 & $\begin{array}{l}457 \\
457\end{array}$ & 840 \\
\hline & Ice Cream, Reach-in & IV5 & -25 & -13 & 1768 & 83 & & \\
\hline \multicolumn{9}{|c|}{$\begin{array}{l}\text { Case refrigeration loads are expressed at Btuh/ft, except for Glass Doc } \\
\text { All refrigeration loads are for stand-alone operation } \\
\text { Electrical power is specified for a } 12 \mathrm{ft} \text { case length or a } 4 \text {-door reachin }\end{array}$} \\
\hline \multicolumn{9}{|c|}{ Multi-deck cases are equipped with shelves unless otherwise noted } \\
\hline \multicolumn{9}{|c|}{ Other case lengths are noted in the description } \\
\hline \multicolumn{9}{|c|}{$\begin{array}{l}\text { Fan wattage is based on standard fans } \\
\text { Light wattage is based on standard configuration used for rating }\end{array}$} \\
\hline Light wattage is basec & on standard configuration used for rating & & & & & & 661-DO & L-010231-1 \\
\hline
\end{tabular}




\subsubsection{Hill Phoenix}

\begin{tabular}{|c|c|c|c|c|c|c|c|c|}
\hline \multirow{2}{*}{ Display Case Type } & \multirow{2}{*}{ Application } & \multirow{2}{*}{$\begin{array}{l}\text { Manufacturer's } \\
\text { Model No. }\end{array}$} & \multicolumn{2}{|c|}{ TEMPERATURES ( $\left.{ }^{\circ} \mathrm{F}\right)$} & \multirow{2}{*}{$\begin{array}{l}\text { Cooling Load } \\
\text { (Btu/hr-ft) }\end{array}$} & \multicolumn{3}{|c|}{ Case Power (Watts) } \\
\hline & & & Evaporator & Discharge Air & & Fans & Lights & Heaters \\
\hline \multirow[t]{59}{*}{ Open Mutu Deck } & Dainy s Deck, $18^{\circ}$ front & OSDM & 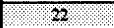 & $34: 3:$ & 1560 & 120 & $\int .92$ & 0 \\
\hline & Dairy, 5 Deck, 21" front & & 22 & 34 & 1518 & 120 & 92 & 0 \\
\hline & Dairy, 5 Deck, 23" front & & 22 & 34 & 1487 & 120 & 92 & 0 \\
\hline & Dairy, 5 Deck, 26 " front & & 22 & 34 & 1456 & 120 & 92 & 0 \\
\hline & Dairy, 5 Deck High, 18 " front & 05DMH & 22 & 34 & 1617 & 150 & 92 & 0 \\
\hline & Dairy, 5 Deck High, 21" front & & 22 & 34 & 1560 & 150 & 92 & 0 \\
\hline & Dairy, 5 Deck High, 23" front & & 22 & 34 & 1529 & 150 & 92 & 0 \\
\hline & Dairy, 5 Deck High, $26 "$ front & & 22 & 34 & 1498 & 150 & 92 & 0 \\
\hline & Dairy, 5 Deck Narrow, 18 " front & 0N5DM & 17 & 32 & 1888 & 150 & 92 & 0 \\
\hline & Dairy, 5 Deck Narrow, 21" front & & 17 & 31 & 1841 & 150 & 92 & 0 \\
\hline & Dairy, 5 Deck Narrow, $27^{\prime \prime}$ front & & 17 & 31 & 1752 & 150 & 92 & 0 \\
\hline & Dairy, 5 Deck High \& Narrow, 18 " front & ON5DMH & 17 & 33 & 1914 & 150 & 92 & 0 \\
\hline & Dairy, 5 Deck High \& Narrow, 21" front & & 17 & 33 & 1867 & 150 & 92 & 0 \\
\hline & Dairy, 5 Deck High \& Narrow, $27 "$ front & & 17 & 32 & 1768 & 150 & 92 & 0 \\
\hline & Dairy, 5 Deck, Rear Loader, 18 " front & O5DR & 17 & 32 & 1562 & 150 & 92 & 0 \\
\hline & Dairy, 5 Deck, Rear Loader, 21" front & & 17 & 32 & 1562 & 150 & 92 & 0 \\
\hline & Dairy, 5 Deck, Rear Loader, 23" front & & 17 & 32 & 1511 & 150 & 92 & 0 \\
\hline & Dairy, 5 Deck, Rear Loader, 26 " front & & 17 & 32 & 1482 & 150 & 92 & 0 \\
\hline & Dairy, 5 Deck, Rear Loader, 21 " front & & 22 & 35 & 1445 & 150 & 92 & 0 \\
\hline & Dairy, 5 Deck, Rear Loader, 23" front & & 22 & 35 & 1479 & 150 & 92 & 0 \\
\hline & Dairy, Deli, Produce, 5 Deck & O5UM & 17 & 30 & 1550 & 120 & 92 & 46 \\
\hline & Deli, 5 Deck, $21 "$ front & OSDM & 17 & 30 & 1633 & 120 & 92 & 0 \\
\hline & Deli, 5 Deck, $23 "$ front & & 17 & 30 & 1591 & 120 & 92 & 0 \\
\hline & Deli, 5 Deck, $26^{\prime \prime}$ front & & 17 & 30 & 1560 & 120 & 92 & 0 \\
\hline & Deli, 5 Deck High, 21" front & OSDMH & 17 & 31 & 1685 & 150 & 92 & 0 \\
\hline & Deli, 5 Deck High, 23" front & & 17 & 31 & 1633 & 150 & 92 & 0 \\
\hline & Deli, 5 Deck High, 26 " front & & 17 & 31 & 1602 & 150 & 92 & 0 \\
\hline & Produce/Beverage, 5 Deck, $18^{\prime \prime}$ front & $05 \mathrm{DM}$ & 27 & 36 & 1550 & 120 & 92 & 0 \\
\hline & Produce/Beverage, 5 Deck, 21" front & & 27 & 36 & 1508 & 120 & 92 & 0 \\
\hline & Produce/Beverage, 5 Deck, $23 "$ front & & 27 & 36 & 1477 & 120 & 92 & 0 \\
\hline & Produce/Beverage, 5 Deck, 26 " front & & 27 & 36 & 1446 & 120 & 92 & 0 \\
\hline & Produce/Beverage, 5 Deck High, 18" front & 05DMH & 27 & 36 & 1555 & 150 & 92 & 0 \\
\hline & Produce/Beverage, 5 Deck High, 21" front & & 27 & 36 & 1513 & 150 & 92 & 0 \\
\hline & Produce/Beverage, 5 Deck High, 23 " front & & 27 & 36 & 1483 & 150 & 92 & 0 \\
\hline & Produce/Beverage, 5 Deck High, 26" front & & 27 & 36 & 1453 & 150 & 92 & 0 \\
\hline & Produce/Beverage, 5 Deck Narrow, 18 " front & 0N5DM & 27 & 37 & 1737 & 150 & 92 & 0 \\
\hline & Produce/Beverage, 5 Deck Narrow, 21" front & & 27 & 36 & 1693 & 150 & 92 & 0 \\
\hline & Produce/Beverage, 5 Deck Narrow, $27 "$ front & & 27 & 36 & 1612 & 150 & 92 & 0 \\
\hline & Produce/Beverage, 5 Deck High \& Narrow, 18" front & 0N5DMH & 27 & 37 & 1768 & 150 & 92 & 0 \\
\hline & Produce/Beverage, 5 Deck High \& Narrow, 21" front & & 27 & 36 & 1706 & 150 & 92 & 0 \\
\hline & Produce/Beverage, 5 Deck High \& Narrow, $27 "$ front & & 27 & 36 & 1659 & 150 & 92 & 0 \\
\hline & Produce/Dairy/Deli, 5 Deck & OSUM & 17 & 30 & 1550 & 120 & 92 & 46 \\
\hline & Dairy/Meat/Deli/Sea food/Produce, 3 Deck & o3UM & 17 & 27 & 1071 & 120 & 92 & 46 \\
\hline & Dairy/Meat/IXeli/Sea food/Produce, 3 Deck, Narrow & ON3UM & 17 & 28 & 857 & 120 & 92 & 46 \\
\hline & Dairy/Meat/Deli/Seafood/Produce, 3 Deck & $025 \mathrm{UM}$ & 17 & 27 & 1061 & 120 & 92 & 46 \\
\hline & Dairy/Meat/Deli/Sea food/Produce, 3 Deck, Narrow & ON25UM & 17 & 28 & 843 & 120 & 92 & 46 \\
\hline & Dairy/Meat/Deli/Sea food/Produce, 2 Deck & O2UM & 17 & 27 & 1040 & 120 & 92 & 46 \\
\hline & Dairy/Meat/Deli/Sea food/Produce, 2 Deck, Narrow & ON2UM & 17 & 28 & 683 & 120 & 92 & 46 \\
\hline & Dairy/Deli/Produce, 4 Deck & O4UM & 17 & 30 & 1518 & 120 & 92 & 46 \\
\hline & Dairy/Deli/Produce, 4 Deck Narrow & ON4UM & 17 & 31 & 1180 & 120 & 92 & 46 \\
\hline & Meat/Deli Multi-deck, 27" front glass, 4-deck & OHM & 17 & 28 & 1506 & 120 & 92 & 100 \\
\hline & Meat/Deli Multi-deck, 33" front glass, 4-deck & & 17 & 28 & 1395 & 120 & 92 & 100 \\
\hline & Meat/Deli Multi-deck, Narrow, 27" front glass & ONHM & 17 & 30 & 1498 & 150 & 92 & 0 \\
\hline & Meat/Deli Multi-deck, Narrow, 33" front glass & & 17 & 30 & 1394 & 150 & 92 & 0 \\
\hline & Meat/Deli, 5 Deck, 27" front glass & OHMH & 17 & 29 & 1492 & 150 & 92 & 200 \\
\hline & Meat/Deli, 5 Deck, 27" front glass & OHMH & 12 & 26 & 1560 & 150 & 92 & 200 \\
\hline & Meat/Deli, 5 Deck, 33" front glass & OHMH & 17 & 29 & 1394 & 150 & 92 & 200 \\
\hline & Deli, Mult-Deck Dome & ONUMD & 17 & 29 & 1446 & 120 & 92 & 47 \\
\hline & $\begin{array}{l}\text { Frozen Food,Multi-deck } \\
\text { Ice Cream }\end{array}$ & $\begin{array}{c}\text { osZ } \\
\text { n/a }\end{array}$ & -17 & -5 & 1914 & 490 & 92 & 562 \\
\hline \multicolumn{9}{|c|}{$\begin{array}{l}\text { Case refrigeration loads are expressed at Btuh/ft, except for Glass Door Reachins which are given in Btuh/door } \\
\text { All refrigeration loads are for stand-alone operation } \\
\text { Electrical power is specified for a } 12 \mathrm{ft} \text { case length or a 4-door reachin }\end{array}$} \\
\hline \multicolumn{9}{|c|}{ Multi-deck cases are equipped with shelves unless otherwise noted } \\
\hline \multicolumn{9}{|c|}{ Other case lengths are noted in the description } \\
\hline \multicolumn{9}{|c|}{ Fan wattage is based on standard fans } \\
\hline \multicolumn{9}{|c|}{ Light wattage is based on standard configuration used for rating } \\
\hline
\end{tabular}


Hill Phoenix (continued)

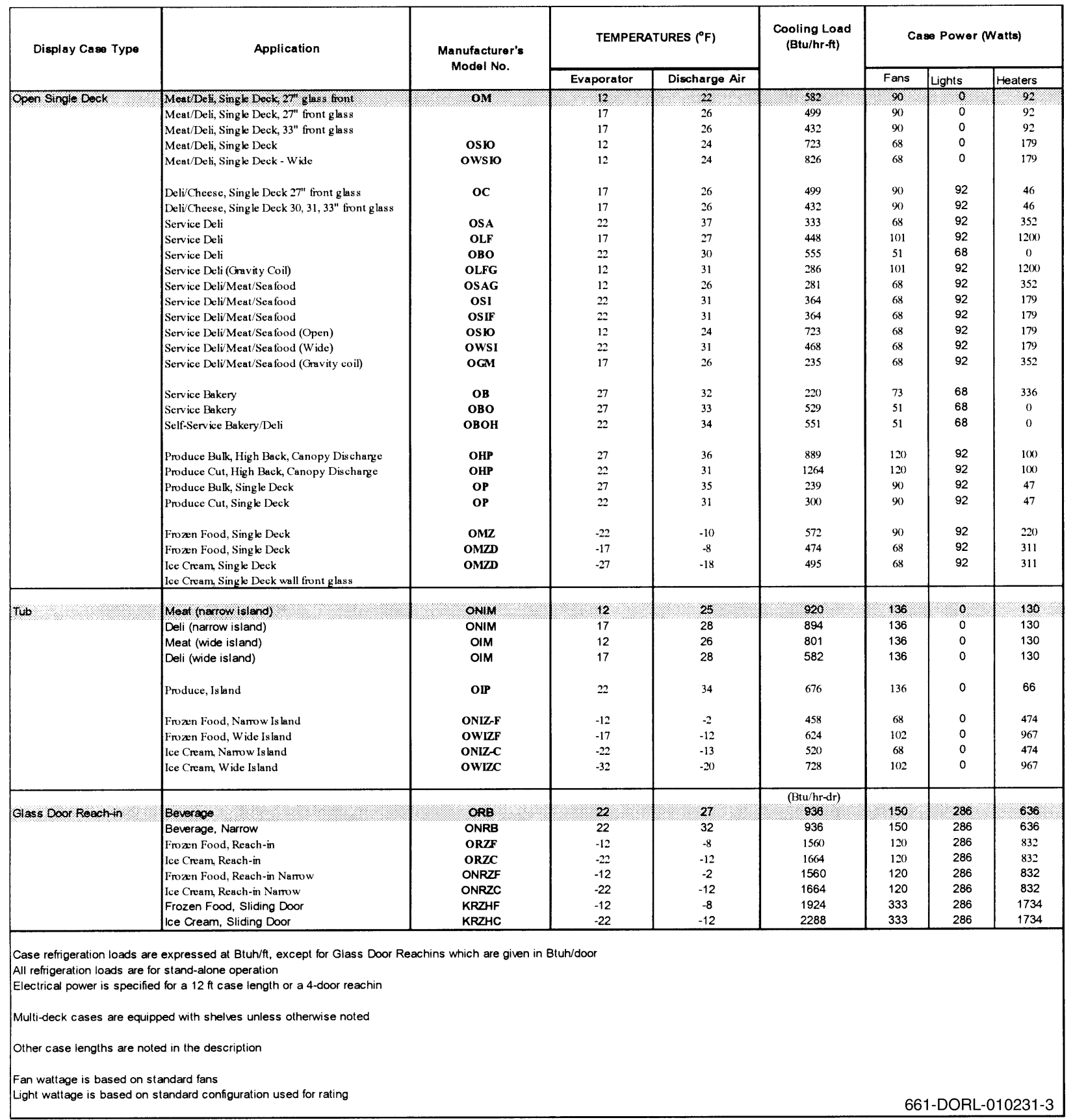




\subsubsection{Hussmann}

\begin{tabular}{|c|c|c|c|c|c|c|c|c|}
\hline \multirow{2}{*}{ Display Case Type } & \multirow{2}{*}{ Application } & \multirow{2}{*}{$\begin{array}{l}\text { Manufacturer's } \\
\text { Model No. }\end{array}$} & \multicolumn{2}{|c|}{ TEMPERATURES $\left({ }^{\circ} \mathrm{F}\right)$} & \multirow{2}{*}{$\begin{array}{l}\text { Cooling Load } \\
\text { (Btu/hr-ft) }\end{array}$} & \multicolumn{3}{|c|}{ Case Power (Watts) } \\
\hline & & & Evaporator & Discharge Air & & Fans & Lights & Heaters \\
\hline \multirow[t]{54}{*}{ Open Muiti-Deck } & bairybeli, 5 -deck standard heght & $\mathrm{DF}$ & 21 & $31:$ & 1500 & 150 & 1085 & $=0$ \\
\hline & Dairy/Deli, 5 Deck, front glass $\left(30^{\prime \prime}\right)$ & D5G & 21 & 31 & 1460 & 150 & 85 & 0 \\
\hline & Dairy/Deli, 5 Deck, high front (27") & DSH & 21 & 31 & 1420 & 150 & 85 & 0 \\
\hline & Dairy/Deli, 5 Deck, low front $\left(19^{\prime \prime}\right)$ & DSL & 21 & 31 & 1580 & 150 & 85 & 0 \\
\hline & Dairy/Deli, 5 Deck, front glass $\left(30^{\prime \prime}\right)$ & DSGE & 24 & 32 & 1410 & 150 & 85 & 0 \\
\hline & Dairy/Deli, 5 Deck, front glass Narrow & D5NGE & 24 & 32 & 1400 & 150 & 85 & 0 \\
\hline & Dairy/Deli, 5 Deck, high front (27") & DSHE & 24 & 32 & 1365 & 150 & 85 & 0 \\
\hline & Dairy/Deli, 5 Deck, high front Narrow & DSNHE & 24 & 32 & 1475 & 150 & 85 & 0 \\
\hline & Dairy/Deli, 5 Deck, low front (19") & DSLE & 24 & 32 & 1515 & 150 & 85 & 0 \\
\hline & Dary/Deli, 5 Deck, low front (19") Narrow & DSNLE & 24 & 32 & 1625 & 150 & 85 & 0 \\
\hline & Dairy/Deli, 5 Deck, standard front (23") & DSE & 24 & 32 & 1440 & 150 & 85 & 0 \\
\hline & Dairy/Deli, 5 Deck, standard Narrow & D5NE & 24 & 32 & 1550 & 150) & 85 & 0 \\
\hline & Dairy/Deli, 5 Deck, Rear Load, low front & D5LR & 21 & 31 & 1740 & 300 & 85 & 0 \\
\hline & Dairy/Deli, 5 Deck,Rear Load, low front & DSLRE & 24 & 32 & 1670 & 300 & 85 & 0 \\
\hline & Dairy/Deli, 5 Deck, Rear Load & DSR & 21 & 31 & 1660 & 300 & 85 & 0 \\
\hline & Dairy/Deli, 5 Deck,Rear Load & DSRE & 24 & 32 & 1595 & 300 & 85 & 0 \\
\hline & Dairy/Deli, 5 Deck, Rear Load, high fiont & DSHR & 21 & 31 & 1480 & 300 & 85 & 0 \\
\hline & Dairy/Deli, 5 Deck,Rear Load, high front & DSHRE & 24 & 32 & 1425 & 300 & 85 & 0 \\
\hline & Dairy/Deli, 5 Deck, low front Narrow & D6L & 21 & 31 & 1660 & 300 & 85 & 0 \\
\hline & Dary/Deli, 5 Deck, low front Narrow & D6LE & 24 & 32 & 1595 & 300 & 85 & 0 \\
\hline & Dairy/Deli, 5 Deck, standard Narrow & D6 & 21 & 31 & 1660 & $3(0)$ & 85 & 0 \\
\hline & Dairy/Deli, 5 Deck, standard Narrow & D6E & 24 & 32 & 1595 & 300 & 85 & 0 \\
\hline & Meat/Deli, 2 Deck & M2E & 21 & 29 & 1055 & 120 & 85 & 0 \\
\hline & Meat/Deli, 2 Deck, front glass & MLEE & 21 & 29 & 915 & 120) & 85 & 0 \\
\hline & Meat/Deli, 3 Deck & $\mathbf{M} 3$ & 18 & 28 & 1205 & 150 & 85 & 0 \\
\hline & Meat/Deli, 3 Deck, front glass & M3G & 18 & 27 & 1060 & 150 & 85 & 0 \\
\hline & Meat/Deli, 3 Deck & MBE & 21 & 28 & 1155 & 150 & 85 & 0 \\
\hline & Meat/Deli, 3 Deck, front glass & MBGE & 21 & 28 & 1020 & 150 & 85 & 0 \\
\hline & Meat/Deli, 4 Deck & M4 & 18 & 28 & 1405 & 30) & 85 & 0 \\
\hline & Meat/Deli, 4 Deck, front glass & M4G & 18 & 28 & 1300 & $3(x)$ & 85 & 0 \\
\hline & Meat/Deli, 4 Deck & MAE & 21 & 29 & 1350 & $3(0)$ & 85 & 0 \\
\hline & Meat/Deli, 4 Deck, front glass & MACE & 21 & 29 & 1250 & 300 & 85 & 0 \\
\hline & Meat/Deli, 4 Deck Narrow & MANE & 21 & 29 & 1630 & 150 & 85 & 0 \\
\hline & Meat/Deli, 4 Deck, front glass Narrow & MANGE & 21 & 29 & 1350 & 150 & 85 & 0 \\
\hline & Meat/DeliDairy/Troduce, 4 Deck & C3LGE & 21 & 30 & 1360 & 150 & 85 & 0 \\
\hline & Meat/Deli/Dairy/Produce, 2 Deck & C2SLE & 21 & 27 & $1000)$ & 117 & 85 & \\
\hline & & & & & & & & \\
\hline & $\begin{array}{l}\text { Meat//TeliDDary//Produce, } 3 \text { Deck } \\
\text { Meat/Deli/Dairy//Produce, 3 Deck }\end{array}$ & C2E & 21 & $\begin{array}{l}27 \\
27\end{array}$ & 1250 & 128 & $\begin{array}{l}85 \\
85\end{array}$ & $\begin{array}{l}0 \\
0\end{array}$ \\
\hline & Meat/Deli/Dairy/Produce, 3 Deck, front glass & C2LGE & 21 & 27 & 11050 & 120 & 85 & 0 \\
\hline & Meat/Deli/Dairy/Produce, 3 Deck, front glass & & 21 & 29 & 1108 & 128 & 85 & 0 \\
\hline & Meat/Deli/Dairy/Produce, 4 Deck & C2XLE & 21 & 29 & 1260 & 128 & 85 & 0 \\
\hline & Meat/DeliDairy/Produce, 4 Deck & C2XE & 21 & 29 & 1190) & 120 & 85 & 0 \\
\hline & Meat/DeliDairy/Produce, 4 Deck glass front & C2XLGE & 21 & 29 & 1155 & 120 & 85 & 0 \\
\hline & Meat/DeliDairy/Produce, 4 Deck & CALE & $21 / 24$ & $29 / 31$ & $1730 / 1570$ & 236 & 85 & 0 \\
\hline & Meat/Deli/Dairy/Produce, 4 Deck & CALGE & $21 / 24$ & $29 / 31$ & $1600 / 1455$ & 236 & 85 & 0 \\
\hline & Meat/Deli/Dairy/Produce, 5 Deck & C5E & $21 / 24$ & $29 / 31$ & 1875/1750 & 300 & 85 & 0 \\
\hline & Meat/Deli/Dair/Produce, 5 Deck & C5GE & $21 / 24$ & $29 / 31$ & 1750/1630 & 300 & 85 & 0 \\
\hline & Meat/Deli/Dairy/Produce, 5 Deck & C5HE & $21 / 24$ & $29 / 31$ & 1855/1730 & 300 & 85 & 0 \\
\hline & Meat/Deli/Dairy/Produce, 5 Deck & CSLE & $21 / 24$ & $29 / 31$ & 1900/1770 & 300 & 85 & 0 \\
\hline & Meat/Deli/Dair/Produce, 5 Deck & & $21 / 24$ & $29 / 31$ & $2070 / 1970$ & 300 & 85 & 0 \\
\hline & Meat/Deli/Dairy/Produce, 5 Deck & C6GE & $21 / 24$ & $29 / 31$ & 1930/1835 & 300 & 85 & 0 \\
\hline & Meat/Deli/Dairy/Produce, 5 Deck & $\mathrm{C} 6 \mathrm{HE}$ & $21 / 24$ & $29 / 31$ & 2040/1940 & 300 & 85 & 0 \\
\hline & Meat/Deli/Dairy/Produce, 5 Deck & C6LE & $21 / 24$ & $29 / 31$ & $2100 / 20000$ & 30) & 85 & 0 \\
\hline & Frozen Meat/Seafood 3-deck & F2XIG & -16 & -2 & 2250 & 171 & 85 & 999 \\
\hline \multirow{2}{*}{\multicolumn{9}{|c|}{$\begin{array}{l}\text { Case refrigeration loads are expressed at Btuh/ft, except for Glass Door } \\
\text { All refrigeration loads are for stand-alone operation } \\
\text { Electrical power is specified for a } 12 \mathrm{ft} \text { case length or a } 4 \text {-door reachin } \\
\text { Multi-deck cases are equipped with shelves unless otherwise noted } \\
\text { Other case lengths are noted in the description }\end{array}$}} \\
\hline & & & & & & & & \\
\hline \multicolumn{9}{|c|}{$\begin{array}{l}\text { Fan wattage is based on standard fans } \\
\text { Light wattage is based on standard configuration used for rating }\end{array}$} \\
\hline
\end{tabular}




\section{Hussmann (continued)}

\begin{tabular}{|c|c|c|c|c|c|c|c|c|}
\hline \multirow{2}{*}{ Display Case Type } & \multirow{2}{*}{ Application } & \multirow{2}{*}{$\begin{array}{l}\text { Manufacturer's } \\
\text { Model No. }\end{array}$} & \multicolumn{2}{|c|}{ TEMPERATURES ( $\left.{ }^{\circ} \mathrm{F}\right)$} & \multirow{2}{*}{$\begin{array}{l}\text { Cooling Load } \\
\text { (Btu/hr-ft) }\end{array}$} & \multicolumn{3}{|c|}{ Case Power (Watts) } \\
\hline & & & Evaporator & Discharge Air & & Fans & Lights & Heaters \\
\hline \multirow[t]{12}{*}{ Open Muth-Deck } & Meatid Defi S Deek & N6 & 18 & 28 & 1640 & 300 & 86 & 0 \\
\hline & Meat Deli, 5 Deck, front glass & MSG & 18 & 27 & $1500)$ & 300 & 85 & 0 \\
\hline & Meat Deli, 5 Deck & MSE & 21 & 29 & 1575 & 30) & 85 & 0 \\
\hline & Meat/Deli, 5 Deck, front glass & MSGE & 21 & 28 & 1440 & $3(x)$ & 85 & 0 \\
\hline & Meat/Deli, 5 Deck Narrow & MSNE & 21 & 29 & 1745 & 150) & 85 & 0 \\
\hline & Meat/Deli, 5 Deck, front glass Narrow & MSNGE & 21 & 29 & 1575 & 150 & 85 & 0 \\
\hline & Produce, High Back & P4 & 21 & 29 & 1650 & $3(x)$ & 85 & 0 \\
\hline & $\begin{array}{l}\text { Produce, } 4 \text { Deck } \\
\text { Prock }\end{array}$ & P4E & 24 & 31 & 1585 & 300 & 85 & 0 \\
\hline & Produce, 4 Deck Narrow & P4NE & 24 & 31 & 1745 & 150 & 85 & \\
\hline & Frozen Food, 6 Deck, high front $\left(30^{\prime \prime}\right)$ & F6H & -18 & -8 & 1485 & 409 & 130 & 570 \\
\hline & Frozen Food, 6 Deck, low front (22") & F6L & -16 & -8 & 1850 & 409 & 130 & 570 \\
\hline & Froxen Food, 6 Deck, standard front (26") & F6 & -16 & -8 & 1570 & $4(9)$ & 130 & 570 \\
\hline \multirow[t]{21}{*}{ Open Single Deck } & MeatDelis Single Deck & Mi & & & & & 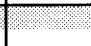 & \\
\hline & $\begin{array}{l}\text { Meat Deli, Single Deck, front glass } \\
\text { Meat Deli, Single Deck }\end{array}$ & $\begin{array}{l}\text { M1G } \\
\text { M1E }\end{array}$ & 18 & 27 & 580 & 128 & 0 & 0 \\
\hline & Meat/Deli, Single Deck, front glass & MIGE & 18 & 26 & 440 & 128 & 0 & 0 \\
\hline & Meat/Deli Service & ED1 & 18 & 27 & 325 & 54 & 170 & 48 \\
\hline & Meat Deti Service & EDS1 & 14 & 24 & 420 & 54 & 0 & 48 \\
\hline & Meat/Deli Service & IEDGI & 14 & 24 & 420 & 234 & 0 & 48 \\
\hline & Meat/Deli Service & SMB & 18 & 24 & 450) & 144 & 170 & 0 \\
\hline & Meat/Deli Service & SMBV & 20 & 25 & 260) & 54 & 170 & 0 \\
\hline & Meat/Deli Service & SMG & 18 & 24 & 450 & 99 & 170 & 0 \\
\hline & Seaf food/Meat/Deli Service & SsG & 18 & 21 & 400 & 45 & 170 & 0 \\
\hline & Produce Bulk, High Back, Canopy Discharge & $\mathbf{P} \mathbf{2}$ & 21 & 37 & 1090 & 120 & 85 & 0 \\
\hline & Produce Bulk, High Back, Canopy Discharge & P2E & 24 & 33 & 1050 & 120 & 85 & 0 \\
\hline & Produce Bulk, Single Deck & P1 & 21 & 31 & 580 & 54 & 0 & 0 \\
\hline & Produce Bulk, Single Deck & PIE & 24 & 31 & 555 & 54 & 0 & 0 \\
\hline & Froren Food, Single Deck wall & L & -20 & -12 & 405 & 54 & 0 & 168 \\
\hline & Froren Food, Single Deck wall front glass & LG & -20 & -12 & 440 & 54 & 0 & 319 \\
\hline & Frozen Food, Single Deck wall - High Front & LGH & -20 & -12 & 440 & 54 & 0 & 288 \\
\hline & Frozen Meat Single Deck & MILGE & .20 & -10 & 550 & 90 & & 120 \\
\hline & Ice Cream Single Deck wall & $\mathbf{L}$ & -30 & -22 & 415 & 54 & 0 & 168 \\
\hline & Ice Cream, Single Deck wall front glass & LG & -30 & .22 & 450 & 54 & 0 & 319 \\
\hline & Ice Cream Single Deck Wall - high front & LGH & -30 & -22 & 450 & 54 & 0 & 288 \\
\hline \multirow[t]{21}{*}{ Tub } & Ding & ns. & 71 & & & & & \\
\hline & Deli/Meat (narrow island) & LNM & 21 & 26 & 36) & 54 & 0 & 96 \\
\hline & Medium Temp Island & CW2GE & 21 & 28 & 1640 & 240) & 170 & \\
\hline & Medium Temp Island & CW2XGE & 21 & 28 & 2060 & 240) & 170 & \\
\hline & Meat (wide island) & MwG & 18 & 26 & 890 & 150 & 0 & 0 \\
\hline & Produce Bulk, Island & PW & 21 & 34 & 1355 & 150 & 0 & 0 \\
\hline & Produce Bulk, Island & PWE & 24 & 34 & 1205 & 150 & 0 & 0 \\
\hline & Frozen Food, Island & LI & -20 & -10 & 475 & 54 & 0 & 168 \\
\hline & Froxen Food, Is land glass & LIG & -20 & -10 & 540 & 54 & 0 & 546 \\
\hline & Frozen Food, Island glass & LIGH & -20 & -10 & 480 & 54 & 0 & 496 \\
\hline & Frozen Food, Namow Is land & LN & -20 & -12 & 405 & 54 & 0 & 168 \\
\hline & Frozen Food, Namow Is land glass & LNG & -20 & -10 & 480 & 54 & 0 & 546 \\
\hline & Frozen Food, Wide Island & LW & -20 & -12 & 675 & 108 & 0 & 336 \\
\hline & Frozen Food, Wide Island glass & LWG & -20 & -12 & 800 & 108 & 0 & 639 \\
\hline & Ice Cream, Island & LI & -30 & .20 & 485 & 54 & 0 & 168 \\
\hline & Ice Cream, Is land glass & LIG & -30 & -20 & 550 & 54 & 0 & 546 \\
\hline & Ice Cream. Is land glass & LGGH & -30 & -20 & 490 & 54 & 0 & 496 \\
\hline & Ice Cream, Narrow Is land & $\mathrm{LN}$ & -30 & .22 & 415 & 54 & 0 & 168 \\
\hline & Ice Cream Narrow Is land glass & LNG & -30 & -22 & 490) & 54 & 0 & 546 \\
\hline & Ice Cream Wide Island & $\mathbf{L w}$ & -30 & -22 & 690) & 108 & 0 & 336 \\
\hline & Ice Cream Wide Island glass & LWG & -30 & .22 & 810 & 108 & 0 & 639 \\
\hline \multirow{9}{*}{ Glass Door Reachin } & & & & & (Btu/hr-dr) & & & \\
\hline & 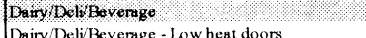 & $\begin{array}{l}\text { RM } \\
\text { RM-Inn }\end{array}$ & $\begin{array}{l}27 \\
27\end{array}$ & 32 & $\begin{array}{l}990 \\
890\end{array}$ & $\begin{array}{l}220 \\
220\end{array}$ & 300 & 215 \\
\hline & $\begin{array}{l}\text { Dairy/Deli Beverage e - Low heat doors } \\
\text { Daniv/DeliB Beverage - Narrow }\end{array}$ & RMN-Inn & 27 & $\begin{array}{l}32 \\
32\end{array}$ & $\begin{array}{l}890) \\
890)\end{array}$ & 220 & 300 & 215 \\
\hline & $\begin{array}{l}\text { Dainy/Deli Beverage · Narrow } \\
\text { Frozen Food, Resch-in } 6 \text { Deck } 4 \text { Doors }\end{array}$ & RL & -11 & -5 & $16 x_{i}$ & 220 & 261 & 1130 \\
\hline & Frown Food, Reach-in 4 Drs - Low heat doors & RL-Inn & -11 & -5 & 1325 & 220 & 300 & 798 \\
\hline & Frozen Food, Reach-in 4 Drs - No heat doors & RL-InnII & -9 & -5 & 1220 & 220 & 300 & 455 \\
\hline & Ife Cream, Reach-in 6 Deck 4 Doors & RL & -19 & -12 & 169\%) & 220 & 261 & 1130 \\
\hline & Ice Cream Reach-in 4 Drs - Low heat doors & RL-Inn & -19 & -12 & 1400 & 220 & 300 & 798 \\
\hline & Ice Cream Reach-in 4 Drs - No heat doors & RL-InnII & -17 & -12 & 1310 & 220 & 300 & 455 \\
\hline \multicolumn{9}{|c|}{$\begin{array}{l}\text { Case reffigeration loads are expressed at Btuh/f, except for Glass Door } R \\
\text { All refrigeration loads are for stand-alone operation } \\
\text { Electrical power is specified for a } 12 \mathrm{f} \text { case length or a } 4 \text {-door reachin } \\
\text { Multi-deck cases are equipped with shelves unlless otherwise noted } \\
\text { Other case lengths are noted in the description }\end{array}$} \\
\hline $\begin{array}{l}\text { Fan wattage is based on st } \\
\text { Light wattage is based on s }\end{array}$ & $\begin{array}{l}\text { andard fans } \\
\text { tandard configuration used for rating }\end{array}$ & & & & & & 61-DOR & 010231-5 \\
\hline
\end{tabular}


Tyler (continued)

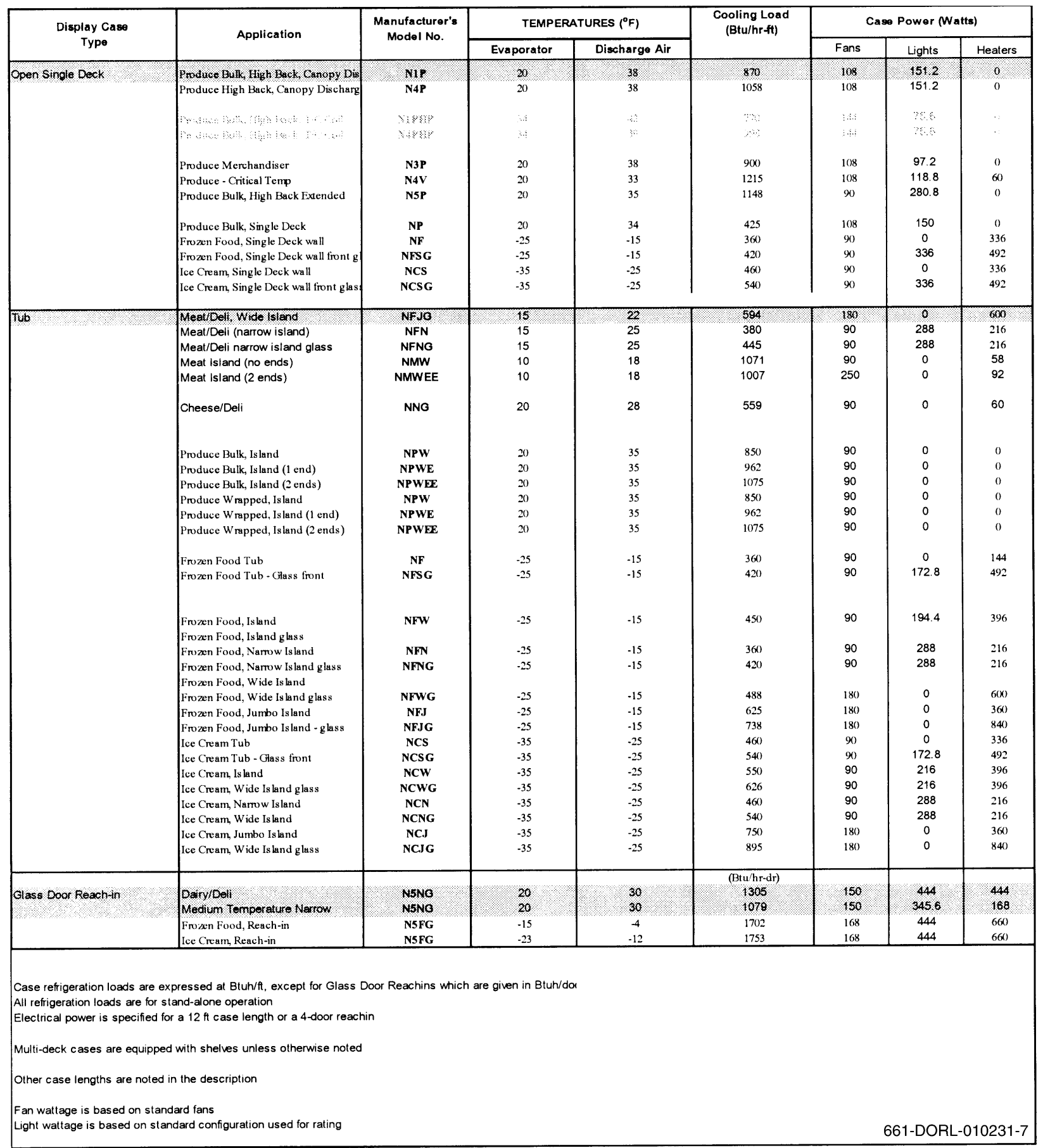




\section{RESULTS FROM THE PROJECT WORKSHOP AND INDUSTRY SURVEYS}

\subsection{Initial Project Workshop}

A workshop was held in Atlanta, GA on January 31, 2001 to provide industry with an overview of the project and to solicit ideas and approaches for improving display case performance. The workshop was well attended by industry representatives, including all major display case manufacturers, engineering personnel from 4 large food chains, and leading researchers in the field of commercial refrigeration.

Several presentations were given including:

- A description of the project plan (D. Walker - Foster-Miller).

- Suggested technologies to investigate (R. Faramarzi - SCE).

- ORNL defrost project (V. Mei - ORNL).

- Case lighting (N. Narendran - RPI).

- EPRI defrost technology field test (M. Khattar - EPRI).

- Display case efficiency issues (G. Kazachki - USEPA).

After the presentations, the attendees broke into 2 working groups to discuss specific topics such as industry involvement in the project, display cases of interest, and technologies and components that should be investigated in the project. Summaries of the group findings were presented at the end of the workshop. Key points that were brought up included the following:

There was strong interest by the case manufacturers to participate in the project, particularly if technologies that were investigated were non-proprietary. Several of the manufacturers indicated willingness to build prototype cases based on design guidelines developed in the project.

Two of the supermarket chains showed interest in possible field testing of the resulting prototype cases.

The display cases that were recommended for investigation were medium temperature, open multi-deck and low temperature reach-in cases. The use of these case types in stores is very prevalent so that improvements would generate significant energy savings. Both groups discussed numerous improvements and technologies of interest. Technologies that were mentioned by both groups included: 
- Improved air curtains and air flow control.

- Advanced defrost techniques using enhanced surfaces or heat transfer methods.

- Better evaporator design.

- The use of electronic flow control valves for the evaporator.

- High-efficiency fan motors and lighting.

- Reduce use of anti-sweat heaters through no-sweat doors or improved heater controls.

At the end of the workshop, each participant was asked to list the top 3 to 4 technologies they felt needed to be addressed to improve display case performance. These were tabulated after the meeting and the results are presented in the following tables. Table 2 provides a ranking from the industry participants only while Table 3 gives an overall ranking considering all participants. Vote totals in the table include votes submitted after the meeting by persons invited but unable to attend. Within the airflow distribution category votes were given for both distribution within the case and across the HX coil. These categories were combined in the rankings below. This was done due to comments during the workshop that both are approaches to help meet product temperature requirements while allowing the maximum possible coil temperature for efficiency purposes.

The industry group gave the most votes (6) to air curtain design with 5 votes each to air flow distribution, HX coil design, and refrigerant flow control components and 4 votes for improved lighting technologies. Defrost controls, improved doors and anti-sweat heaters, and cleanliness/ case cleaning issues were given second priority with other individual items receiving one vote each.

The overall rankings are a bit more diverse but the general ordering of the technology options is about the same. Airflow distribution is the top-ranked issue with 10 votes total. Air curtain design, HX coil design, refrigerant flow controls, defrost controls, and improved lighting

Table 2. $\quad$ Industry ranking of display case improvement technologies

\begin{tabular}{|l|c|}
\hline \multicolumn{1}{|c|}{ Technology } & No. Votes \\
\hline Air Curtain Design & 6 \\
\hline Air Flow Distribution & 5 \\
- Within Case & $(3)$ \\
- Across HX Coil & $(2)$ \\
\hline HX coil design & 5 \\
\hline Refrigerant Flow Controls & 5 \\
(includes TXVs, electronic XVs, distributors, electronic EPR valves, etc.) & \\
\hline Lighting & 4 \\
\hline Defrost Controls & 3 \\
\hline Doors (including anti-sweat heaters) & 3 \\
\hline Cleanliness/Self-Cleaning Cases & 2 \\
\hline Product facings (merchandising issues) & 1 \\
\hline Case-to-Case Joining & 1 \\
\hline Insulation & 1 \\
\hline Better Fan Motors & 1 \\
\hline Case Controller & 1 \\
\hline
\end{tabular}


Table 3. Overall ranking of display case improvement technologies

\begin{tabular}{|l|c|}
\hline \multicolumn{1}{|c|}{ Technology } & No. Votes \\
\hline $\begin{array}{l}\text { Air flow distribution } \\
\text { - Within Case }\end{array}$ & 10 \\
- Across HX Coil & $(6)$ \\
\hline Air Curtain Design & 7 \\
\hline HX Coil Design & 7 \\
\hline $\begin{array}{l}\text { Refrigerant Flow Controls } \\
\text { (includes TXVs, electronic XVs, distributors, electronic EPR valves, etc.) }\end{array}$ & 7 \\
\hline Defrost controls & 6 \\
\hline Lighting & 6 \\
\hline Doors (including anti-sweat heaters) & 4 \\
\hline Cleanliness/Self-Cleaning Cases & 2 \\
\hline Better Fan Motors & 2 \\
\hline Case Controller & 2 \\
\hline Product Facings (merchandising issues) & 1 \\
\hline Case-to-Case Joining & 1 \\
\hline Insulation & 1 \\
\hline Better Defrost Technologies & 1 \\
\hline Define Efficiency & 1 \\
\hline
\end{tabular}

comprise the second ranked group with 6 to 7 votes each. Improved doors received 4 votes while other items received one or two votes each.

After the workshop, several attendees indicated that striving for a 10 to 20 percent reduction in case refrigeration load and parasitic power consumption seemed a reasonable goal.

\subsection{Survey on Test Case Selection}

As part of the planning for the baseline display case testing and for the lab work associated with the air curtain development, a survey of industry representatives was performed to determine which case types should be used for this initial lab testing. The display cases initially selected for this investigation consist of:

- Open multi-deck meat case.

- Open multi-deck dairy/deli case.

- Low temperature reach-in case (optional).

These particular cases were selected because open, multi-deck medium temperature display cases represent about half of the refrigerated fixtures in typical supermarkets (2). Low temperature reach-in cases are also widely used, typically accounting for about one-third of fixtures (2) and have a large impact on total refrigeration energy use but were ranked lower in priority by our industry advisors for this project.

The individuals contacted consisted of attendees of the Atlanta workshop and industry members previously involved in DOE/ORNL projects dealing with supermarket refrigeration. These people were contacted and asked for their opinion on the display cases chosen for the 
investigation and also for any other recommendations they may have concerning test case selection.

Table 4 gives the results of the survey. The 8 people that responded were unanimously in favor of the display cases and case types chosen. Interest was also expressed in two other case types, single-deck meat, and multi-deck produce.

Table 4. Results of industry survey of test display case selection

\begin{tabular}{|l|l|l|l|}
\hline \multicolumn{1}{|c|}{ Respondent } & \multicolumn{1}{|c|}{$\begin{array}{c}\text { Company/ } \\
\text { Organization }\end{array}$} & \multicolumn{1}{c|}{ Industry Affiliation } & \multicolumn{1}{c|}{ Comment/Recommendation } \\
\hline Sandy Sandahl & Target & Store Operator & In favor of cases selected \\
\hline Josh Imig & ARI & Researcher & In favor of case selected \\
\hline Bill Wehmeier & Alco & Valve Manufacturer & In favor of cases selected \\
\hline Dean Swofford & Hill-Phoenix & Case Manufacturer & In favor of cases selected \\
\hline Steve Sloan & Publix & Store Operator & $\begin{array}{l}\text { In favor of cases selected; also interested } \\
\text { in single-deck meat cases }\end{array}$ \\
\hline John Behr & Hussmann & Case Manufacturer & $\begin{array}{l}\text { Agreed with investigation of multi-deck } \\
\text { medium temperature cases; remarked on } \\
\text { significance of low temperature reach-ins } \\
\text { because of lower EER seen with low } \\
\text { temperature systems. Recommended } \\
\text { investigating 5-deck dairy/deli case }\end{array}$ \\
\hline Dave Meninger & Kroger & Store Operator & $\begin{array}{l}\text { Agrees with investigation of medium } \\
\text { temperature multi-deck; also interested in } \\
\text { multi-deck produce }\end{array}$ \\
\hline Joe Humphrey & Tyler & Case Manufacturer & In favor of cases selected \\
\hline
\end{tabular}

\subsection{Survey from the Food Marketing Institute (FMI) Energy Conference}

A survey was conducted at the Food Marketing Institute Energy Conference held in Cleveland, $\mathrm{OH}$ on September 9 to 12, 2001 to gather ideas and comments from supermarket operators on the project. Mr. Ramin Faramarzi from Southern California Edison gave a series of presentations on recent work on display case done at his laboratory. At the beginning of session, attendees were asked to fill out a survey. The questions contained in the survey are included in Table 5, which also provided a summary of the responses.

A total of 20 individuals answered the survey questions. Each respondent provided multiple answers to each question; the results given in the table include all answers given by the respondents.

The responders identified multi-deck display cases, low or medium temp, as the least efficient case types. A significant number of votes were also given for low temp coffin and wide island tub cases. The energy technology most mentioned in the survey was improved lighting followed by air curtain and evaporator improvements (including electronic control valves). High-efficiency fan motors and no-heat doors each got 2 votes in this category. 
Table 5. Summary of responses from the Display Case Survey, Food Marketing Institute Energy Conference, Cleveland, OH, September 9 to 12, 2001

\begin{tabular}{|c|c|}
\hline \multicolumn{2}{|c|}{$\begin{array}{l}\text { Which types of refrigerated display cases do you consider least energy efficient? Please list maximum } \\
\text { of three. }\end{array}$} \\
\hline $\begin{array}{c}\text { Case Type Identified } \\
\end{array}$ & No. of Responses \\
\hline Medium Temperature Multi-Deck & 12 \\
\hline Low Temperature Multi-Deck & 11 \\
\hline Low Temperature Coffin & 5 \\
\hline Wide Island Tub & 4 \\
\hline Self-Contain ed & 3 \\
\hline Single-Deck Meat & 2 \\
\hline Specialty Cases & 2 \\
\hline Low Temperature Reach-Ins & 1 \\
\hline \multicolumn{2}{|c|}{$\begin{array}{l}\text { What energy efficiency technologies should be investigated to improve the performance of the } \\
\text { suggested display cases? }\end{array}$} \\
\hline Technology & No. of Responses \\
\hline Improved Lighting & 6 \\
\hline Air Curtain Improvement & 5 \\
\hline Improved Evaporator Coil & 3 \\
\hline Electronic Control Valves & 2 \\
\hline No-Heat Doors & 2 \\
\hline High-Efficiency Fan Motors & 2 \\
\hline Night Covers & 1 \\
\hline Improved Wall Insulation & 1 \\
\hline Air Shelves & 1 \\
\hline Door on Medium Temperature Cases & 1 \\
\hline
\end{tabular}

The response to the medium temperature door cases was evenly divided between those favoring and not favoring their use. Many of the respondents indicated that their merchandisers fear loss of sales with doors installed. Only 2 indicated that energy savings might be a factor in encouraging their use.

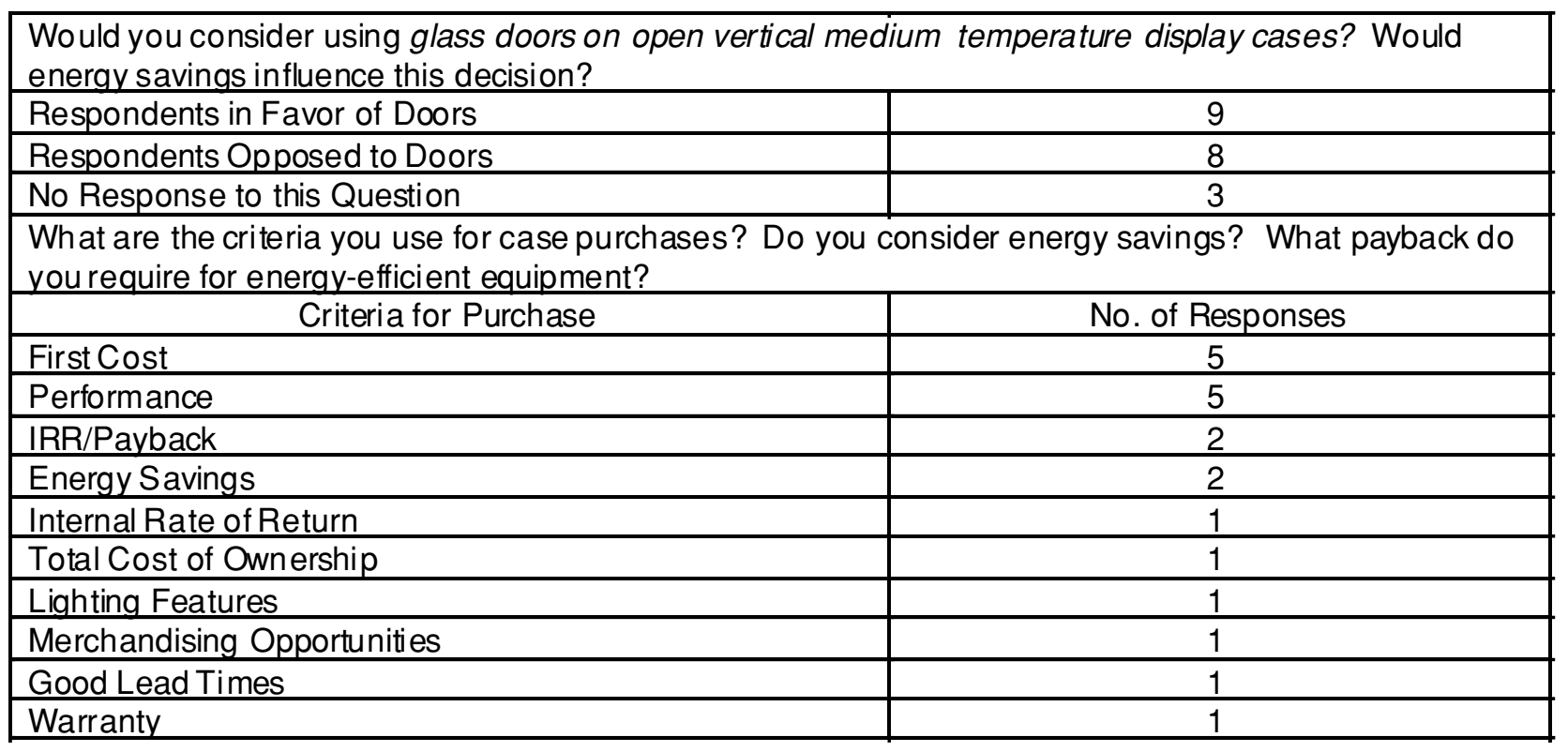


First cost and performance were indicated as leading criteria for case purchase. Performance would be the most significant factor if the energy savings responses were included in this count. Simple payback was the preferred method of evaluation. Other methods included internal rate of return and total cost of ownership. Four respondents listed other criteria such as merchandising, lighting features, warranty, and short lead time for delivery as significant in display case selection.

\subsection{Second Project Workshop}

The second project workshop for the high-efficiency refrigerated display case project was held on Wednesday, January 29, 2003 in Chicago, IL. The workshop was held in conjunction with the Chicago winter ASHRAE meeting that took place prior to the workshop. The purpose of the workshop was to present project results to representatives of the supermarket and commercial refrigeration industries and to gather recommendations as to how best to proceed from this point.

\subsubsection{Workshop Agenda}

Table 6 shows the agenda for the workshop.

Table 6. Agenda for the high-efficiency supermarket display case project industry workshop

\begin{tabular}{|l|l|}
\hline \multicolumn{1}{|c|}{ Introduction } & \\
\hline Case Technology Selection Energy Analysis & Ramin Faramarzi \\
& Southern California Edison RTTC \\
\hline Draft Rating Procedure & David Walker \\
& Foster-Miller, Inc. \\
\hline Discussion by Group & Review of Work \\
& Comments on Ratings \\
& Recommend Future Efforts \\
\hline
\end{tabular}

\subsubsection{Case Efficiency Improvement Technology Selection and Energy Analysis Results (Ramin Faramarzi - Southern California Edison RTTC)}

The objective of this research project is to promote energy-efficiency solutions in refrigerated display cases, while enhancing food safety without hampering merchandizing facets. The project will attempt to: establish the baseline performance of existing display cases; identify available and near-term technologies that reduce display case load and direct energy use; establish potential energy savings through analysis and testing; and demonstrate these savings in a prototype high-efficiency display case. Results of the project will be disseminated to the industry.

The project employs a combination of test and analytical methods to evaluate efficiency enhancing methods (EEMs) for display cases. Testing of display cases follows procedures specified in ASHRAE 72-98. Air curtain testing involves the use of digital particle image 
velocimetry (DPIV) to measure curtain airflow and also to calibrate a computational fluid dynamics (CFD) model that will be used to investigate ambient air entrainment in the case air curtain. Analytical modeling of the EEMs is also undertaken using a version of DOE-2.3 that is calibrated with field monitored data from an operating supermarket. The most influential EEMs were identified from this combination of analytical and test results. The design of the prototype case will incorporate these selected EEMs.

A survey of supermarkets in the Southern California Edison service territory shows that approximately 50 percent of the cases employed are open, vertical multi-deck type. Low temperature reach-ins is the next largest group at 33 percent. The open multi-deck display cases also represent the largest portion of the total refrigeration load. Testing at RTTC has shown that approximately 80 percent of the refrigeration load of a multi-deck display case can be attributed to ambient air entrainment.

Criteria for the selections of EEMs to evaluate were the following:

- All technologies evaluated are mature enough for use in commercial equipment or will be in the near term.

- EEMs must have potential for energy savings or enhancement of product temperature maintenance.

- The first costs of the EEMs were not a major factors in their selection.

The EEMs selected for evaluation fell into 3 categories:

Evaporator Coil Assembly

- Modular coil and defrost.

- High-efficiency evaporator and advanced controls (Electronic Expansion Valve (EEV) and Controlled Discharge Step-Motor (CDS)).

- Liquid-suction heat exchanger.

Air Distribution and Curtain System

- Electronically commutated (ECM) fan motor.

- Scroll cage fan with ECM motor.

- Improved air curtain.

- Glass doors on open vertical medium temperature cases.

Lighting System

- Fluorescent - type T8EB with enhanced interior film. 
- Fluorescent - type T5EB with enhanced interior film.

- Day lighting system.

The analysis work for the EEMs was directed toward medium temperature multi-deck and island cases, and low temperature reach-in cases. For testing, only medium temperature multideck cases were investigated.

The DPIV measurement technique was used to visualize the flow path of a multi-deck display case air curtain and to map the air velocity field of the air curtain. The air velocity map was employed to calibrate a CFD model of the display case. The CFD model was then used to investigate ambient air entrainment in the air curtain.

CFD results suggest that the amount of ambient air entrained by the discharge air curtain is closely related to the turbulence intensity of the air curtain at the discharge air grill, where the intensity, $\mathrm{i}$, is defined as

$$
\mathrm{i} \equiv \frac{\text { velocity fluctuations }}{\text { mean velocity }}
$$

Analysis results suggest that complete elimination of turbulence at the discharge air grill will reduce ambient air entrainment by 15 percent.

Turbulence intensity and entrainment can also be related to the Reynolds number of the air curtain, where the characteristic dimension is the width of the discharge air grill. The display case tested has an air curtain Reynolds number of approximately 4200 and a turbulence intensity of 18 percent (average operating conditions). The desired air curtain Reynolds is on the order of 3200 , which will result in a turbulence intensity of 2 percent.

Preliminary results from the DPIV measurements and CFD modeling indicate that the total airflow through the display case evaporator is approximately $780 \mathrm{ft}^{3} / \mathrm{min}$. The airflow at the discharge air grill is estimated at $280 \mathrm{ft}^{3} / \mathrm{min}$, while the remainder of the flow $\left(500 \mathrm{ft}^{3} / \mathrm{min}\right)$ passes through the back panel. The total ambient airflow that is entrained in the air curtain is $325 \mathrm{ft}^{3} / \mathrm{min}$, or 42 percent of the total fixture airflow; however, the net infiltrated flow that reaches the case evaporator is $100 \mathrm{ft}^{3} / \mathrm{min}$, or 13 percent of the total fixture airflow. The entrained air mixes with the curtain air, and then a large portion of this mixed air spills out of the case.

The preliminary conclusions derived from the DPIV measurements and the CFD modeling are the following:

- Critical factors affecting the performance of the air curtain are:

- Reynolds number characterized by the air grill width.

- Turbulence intensity at the air grill.

- Discharge air velocity (or entering jet) profile. 
- Minimizing number of velocity peaks in the center of the discharge air grill to one peak will lower turbulence intensity, thereby, reducing mixing and entrainment at the grill.

- Minimizing velocity fluctuations and approaching a laminar region will reduce turbulence intensity at the walls and other locations.

(Full details of the air curtain investigations and development of the $1^{\text {st }}$ generation improved air curtain design are contained in Section 8 of this report.)

The EEMs for the display cases were investigated using a DOE-2.3 simulation of a supermarket developed by SCE RTTC. The DOE-2.3 supermarket model was calibrated using field test data gathered at a supermarket in SCE service territory.

Simulation results for a supermarket in Los Angeles showed that improved case air curtains and the use of doors on medium temperature cases produced the greatest change in refrigeration load, reducing the load by 199 and $605 \mathrm{kBtu} / \mathrm{hr}$, respectively. Combining the improved air curtains with the high efficiency evaporator, ECM fan motors, and improved lighting resulted in a load reduction of $418 \mathrm{kBtu} / \mathrm{hr}$. Combining these same measures with glass doors resulted in a load reduction of $794 \mathrm{kBtu} / \mathrm{hr}$. Measures producing greatest savings in refrigeration energy were glass doors on medium temperature cases, improved air curtains, and ECM fan motors. Combined measures with improved air curtains resulted in annual energy savings of 219,138 kWh; combined measures with glass doors gave savings of 417,748 kWh.

The modeling was performed for the following 5 additional locations:

- Chicago, IL.

- Houston, TX.

- Knoxville, TN.

- Seattle, WA.

- Washington, DC.

The results of the modeling showed that the greatest reduction in refrigeration load was achieved by the combined EEMs with glass doors on medium temperature display cases. The largest reduction was seen for a store in Houston and the smallest for a store in Chicago. Greatest energy savings were estimated for a store in Seattle, while the smallest savings were seen in a store in Chicago. (More complete details of the DOE-2.3 simulation study are included in Section 7 of this report.)

Laboratory testing was conducted on a baseline multi-deck medium temperature display case. The display case was tested using the procedure outlined in ASHRAE standard 72-98. Table 7 gives a comparison of case manufacturer's and test data.

A comparison was made of the baseline case's infiltration airflow estimated by the CFD model and the airflow determined from the test measurements. The CFD model set the infiltration flow at $100 \mathrm{ft}^{3} / \mathrm{min}$, while the test measurements suggest an infiltration flow rate of 
Table 7. Comparison of test and manufacturer's published data for the test multi-deck medium temperature display case

\begin{tabular}{|l|c|c|}
\hline & Manufacturer Data & Test Results \\
\hline Average Cooling Load during 3/4 of Run Cycle $(\mathrm{Btu} / \mathrm{h} / \mathrm{ft})$ & 1,561 & 1,624 \\
\hline Average Saturated Evaporator Temperature $\left({ }^{\circ} \mathrm{F}\right)$ & 17 & 17 \\
\hline Average Superheat Set Point $\left({ }^{\circ} \mathrm{F}\right)$ & 6 to 8 & 10 \\
\hline Average Discharge Air Temperature $\left({ }^{\circ} \mathrm{F}\right)$ & 28 & 30 \\
\hline Average Product Temperature $\left({ }^{\circ} \mathrm{F}\right)$ & 35 & 33 \\
\hline Average Return Air Temperature $\left({ }^{\circ} \mathrm{F}\right)$ & 38 & 37 \\
\hline Average Post Defrost Discharge Air Velocity $(\mathrm{fpm})$ & $\mathrm{N} / \mathrm{A}$ & 186 \\
\hline Peak Post Defrost Discharge Air Velocity (fpm) & 350 & 292 \\
\hline
\end{tabular}

$117 \mathrm{ft}^{3} / \mathrm{min}$. (Complete details of the air curtain and case performance test setup, data analysis procedures, and test results are included in Section 6 of this report.)

The breakdown of the refrigeration load on the display case is estimated as:

- Conduction $-526 \mathrm{Btu} / \mathrm{hr}$.

- Radiation $-1,016 \mathrm{Btu} / \mathrm{hr}$.

- Infiltration - 10,674 Btu/hr.

- Internal generation (lights and evaporator fans) $-773 \mathrm{Btu} / \mathrm{hr}$.

\subsubsection{Draft Rating Procedure for High-Efficiency Display Cases (David Walker - Foster- Miller, Inc.)}

The purpose of the efficiency rating procedure is to provide recognition for display cases with better performance than the present baseline. The results of the rating procedure are similar to that obtained through programs like Energy Star, but Energy Star only recognizes the best presently available cases and does not allow for future improvements which would result in the highest potential savings possible. The suggested rating procedure considers the performance of the display case only and does not include compressor system performance.

The test procedure used in developing the data needed for the case rating follows that same procedures described in ASHRAE standards 72 and 117. Using these procedures provides uniform report results at the same prescribe operating conditions. Another significant standard that will be employed in developing the rating procedure is ARI standard 1200. In order for a display case to be considered for performance rating, the case must meet all pertinent health and sanitation codes. The display case must also be able to maintain food storage temperature as described in the FDA food code.

Ratings will be performed on each case based upon the configuration of the case and its storage application. Table 8 shows possible sets of case configuration and applications. 
Table 8. Configuration and storage categories

for the case rating procedure

\begin{tabular}{|l|l|}
\hline Configuration & Storage Application \\
- Multi-Deck & Dairy \\
- - 3 to 5 decks & Deli \\
- Single Deck & - Meat \\
- Tub & - Broduce \\
- Coffin or Wide Island & - Frozerage \\
- Service & - Ice Cream \\
- Glass Door Reach-In &
\end{tabular}

The basis of comparison for the rating will be to compare the performance of the candidate display case with a baseline case of similar configuration and storage application. The baseline case will be a composite of presently available cases that will be formulated from manufacturer's published data. A set of metrics will be calculated where the candidate case is compared to an "ideal" case having the highest efficiency possible as determined through analysis (i.e., a combination of analytical and measured results from this project). A score will be assigned for each metric depending upon how close the candidate case comes to the theoretical ideal case. The score for each metric is then added together and the candidate case must reach a predetermined minimum score in order to be considered "high-efficiency."

A possible set of metrics for the display case rating is listed in Table 9. The major elements are reduction in refrigeration load, improved evaporator performance, reduced installed electrical load, and reduction in refrigerant charge for case operation. This final category is not an energysaving measure, but has a favorable environmental impact. (The draft rating procedure is discussed more fully in Section 9.)

Table 9. Proposed metrics for the draft display case efficiency rating procedure

\begin{tabular}{|c|c|c|}
\hline Refrigeration Load & \% Reduction in Baseline Load & Goal $-50 \%$ Reduction \\
\hline Evaporator Performance & $\%$ Decrease in Tair - Tevap (DT) & Goal $-\mathrm{DT}=4^{\circ} \mathrm{F}$ \\
\hline $\begin{array}{l}\text { Electric Loads } \\
\text { - Fans } \\
\text { - Case lights } \\
\text { - Anti-sweat heaters }\end{array}$ & $\begin{array}{l}\text { \% Reduction in electric power and } \\
\text { contribution to load reduction }\end{array}$ & Goal - 70\% Reduction \\
\hline Environmental & \% Reduction in case refrigerant charge & Goal $-25 \%$ reduction \\
\hline
\end{tabular}

\subsubsection{Workshop Discussion Results}

An open discussion was held at the end of the workshop to gather comments on work performed to date and also list recommendations for future work on the project.

Work to Date

- Test Methodology and Error Analysis - It was suggested that a detailed test plan and error analysis be prepared for the prototype display case testing. 
- Interactive impacts on design - It was pointed out by several that there would be a significant interaction in the design of the display case air curtain and the evaporator. An iterative process will be required to determine the proper airflow for the air curtain to minimize air entrainment while achieving correct air-side heat transfer in the evaporator.

- DOE-2.3 store simulation results for the high-efficiency evaporator - The energy savings produced by operating with a smaller coil TD (discharge air - evaporator) appear too small. Greater energy savings are expected. This result should be checked.

- Include cost as factor - The cost of the EEMs should be included in the evaluation.

- Case clean ability - NSF and other codes relating to the sanitation and clean ability of the display case should be included in the design of the prototype case.

- Floating suction pressure control - The use of floating suction pressure control should be evaluated to determine the potential energy savings achievable by the use of this control strategy.

- Limit to air curtain reduction - The limit to the reduction in air entrainment was discussed and CFD modeling will be used to determine this limit.

- Liquid-suction heat exchanger - More details were requested about the liquid-suction heat exchanger listed as part of the enhancement of the display case coil, particularly how large the exchanger is and how much pressure drop is incurred to the suction-side of the display case.

Future Work

- Total Airflow of the display case - A question was raised about modeling predictions and test results for total case airflow. It was agreed that retesting of the case would be done to verify the airflow result.

- Draw-through airflow testing - It was suggested to test the case evaporator with drawthrough airflow to see if more uniform airflow and discharge temperature can be obtained.

- Integration of the CFD and evaporator model results.

- Influence of back panel airflow should be evaluated.

\section{Ratings}

- $\quad$ Ratings procedure should be integrated with ARI 1200.

- Category Weightings - Category weightings should be changed for each display case type. 


\subsubsection{Attendees List}

Table 10 lists the attendees of the second January 2003 workshop for the high-efficiency supermarket display case project.

\section{Table 10. Attendee list for the advanced supermarket display case workshop, January 2003}

\begin{tabular}{|l|l|}
\hline \multicolumn{1}{|c|}{ Name } & \multicolumn{1}{c|}{ Organization } \\
\hline Homayun Navaz & Kettering University \\
\hline Scott Martin & Hill Phoenix \\
\hline Dean Swofford & Hill Phoenix \\
\hline Robert Tanner & Hill Phoenix \\
\hline Jim Sand & Oak Ridge National Lab \\
\hline Hugh Henderson & CDH \\
\hline Rusty Walker & Hill Phoenix \\
\hline Henry Pellerin & Hill Phoenix \\
\hline David Hinde & Hill Phoenix \\
\hline John Rasch & Hussmann \\
\hline John Roche & Hussmann \\
\hline Georgi Kazachki & Hill Phoenix \\
\hline Clark Bullard & U of Illinois ACRC \\
\hline Al Maier & Emerson Flow Control \\
\hline Bill Weihmeier & Emerson Flow Control \\
\hline John Murray & Sporlan Valve \\
\hline Mike Kempiak & Tyler \\
\hline
\end{tabular}

\subsection{Third Project Workshop - Southern California Edison, Irwindale, CA - January 22, 2004}

\subsubsection{Purpose and Background}

The meeting had two purposes. First was to brief our supermarket industry advisors on the results of tests on a prototype advanced display case. The case design is the result of a DOEsponsored laboratory testing and analysis project conducted over the past two and a half years. This work, focused on open-front, multi-deck medium temperature case types, involved prioritization and detailed evaluation of several efficiency enhancement measures including redesign of the case air curtain to reduce ambient air entrainment. The prototype case fabrication and testing is the culmination of these efforts.

The second purpose was to solicit industry feedback on the work to-date and input on future project activities. Industry feedback received in all of the workshop held during this project has been of great help in focusing our efforts. We continue to need their input and guidance on where to focus our future work. None of what we do will mean anything if it is not useful to the supermarket industry - the primary ultimate user of this technology.

Our goals for the project (established after the first workshop) were to reduce the refrigeration load by 10 to 20 percent and to reduce energy consumption by at least the same amount. 


\subsubsection{Case Efficiency Improvement Technology Selection and Energy Analysis Results}

After introductions, there were two presentations made to the group by Ramin Faramarzi and by Dr. Navaz.

Ramin's presentation summarized the project plans, development of the prototype design, and comparison of baseline and prototype case test results up through January 21. Both the prototype and baseline cases were open front, four deck medium temperature units for deli, meat, and dairy display that were each $8 \mathrm{ft}$ in length. The prototype and baseline look alike (intentionally) but the prototype incorporates a number of efficiency enhancement features that were evaluated during the design development phase of the project. Prototype construction was a collaborative effort between Southern California Edison (SCE), Hill Phoenix, Wolverine Tube, and the University of Illinois Urbana/Champaign. Efficiency improvement features included in the prototype included the following:

- A more efficient evaporator coil featuring greater airside surface area (higher fin density) and enhanced surfaces on the inside of the refrigerant tubes.

- Super T8 lamps.

- The original three propeller fans used to circulate air through the case evaporator coil, food display area, and air curtain were replaced with two tangential blower wheels to improve the coil airflow distribution.

- The original three PSC fan motors were replaced with a single electronically commutated motor (ECM) with a variable-speed drive (VSD). The VSD enabled the coil CFM to be held constant throughout the time between defrosts. It also enabled over speeding during the off-cycle defrost to reduce defrost time/cycle.

- A dual-port TXV was used.

- A high-effectiveness liquid/suction heat exchanger (LSHX) was incorporated to provide for increased refrigerant liquid subcooling before the expansion valve. A micro channel design was used for the LSHX.

- Modifications were made to the geometry of the air curtain discharge air grill (DAG) in an attempt to reduce infiltration of store air into the evaporator air stream and thereby reduce the case refrigeration load.

Initial prototype case test results (as of January 22, 2004) show the following:

- Total prototype case $\mathrm{kW}$ reduced 27 percent compared to baseline and

- Prototype case refrigeration load reduced 2 percent compared to baseline. 
Ramin noted that the product temperatures achieved by the prototype during these tests (both average and maximum) were about $2^{\circ} \mathrm{F}$ higher than for the base case. In addition, the maximum product temperature for the prototype case was $41.5^{\circ} \mathrm{F}$, which exceeds the $41^{\circ} \mathrm{F}$ maximum allowable. Therefore additional testing was conducted after the workshop (see subsection 10.3) with case control parameters adjusted to match the product temperature of the baseline case. It was also noted that the load reduction was much lower than we had hoped to obtain.

Dr. Navaz' presentation discussed the considerable work done on testing and analysis of the case air curtain. Laser Doppler velocimetry (LDV) and digital particle image velocimetry (DPIV) techniques were used to measure the air curtain flow field and to calibrate a CFD model of the field. The calibrated CFD model was used to determine what parameters had the most impact on store air infiltration. Minimization of the Reynolds number and turbulence intensity (both as determined at the DAG) was determined to be key to minimizing air infiltration. Simulation of a number of geometries was conducted in order to develop the proposed design that was implemented in the prototype case. The geometry used was predicted to have a velocity profile that was approximately parabolic with a maximum close to the inside (food side) of the DAG. However, the tests completed showed that the velocity peak was actually at the outside (store side) of the DAG. It was also found during tests of the prototype case that lowering the air curtain Reynolds number (and, therefore air velocity) caused a significant drop in the momentum of the air curtain causing it to spread out and lose its integrity before reaching the return air grill. The air drawn into the case was mixture of air taken from the ambient and the display case, resulting in substantial infiltration of ambient air. It is therefore necessary to determine the minimum air velocity needed to maintain the air curtain across the display case and factor that into the velocity profile at the DAG.

Using the prototype test results, the CFD model was further tuned and the DAG geometry was revaluated. As a result of this effort, a revised geometry has been proposed for the DAG to achieve the desired velocity profile and reduced turbulence levels needed to minimize air infiltration to the case coil.

Bottom line - The prototype case had met the energy reduction goals but not the load reduction goals as of the date of this meeting.

\subsubsection{Discussion Session}

After lunch and a tour of the SCE Refrigeration and Thermal Test Center (RTTC) laboratory to inspect the prototype case, a discussion session was held. The questions below had been posed to the workshop invitees prior to the meeting.

Prior to beginning work on the advanced display case project, a planning workshop was held in January 2001 to seek advice and guidance from supermarket company and manufacturer representatives. A list of priorities was developed which we have generally followed during our case analyses and testing work to date. The prototype case and its lab tested performance have been the result. However, unless advanced cases which implement some or all of the technologies of the prototype are used in future supermarkets, the work will remain of academic 
interest only. Therefore, we need your advice on best strategies to promote use of cases like the prototype.

- Are the lab test results enough to influence you to install advanced cases?

- Would a field demonstration be necessary to convince supermarket decision makers to specify advanced cases?

- How should a field test best be structured?

- Side-by-side stores (one with advanced cases, one with standard)?

- Advance case lineup and standard case lineup on separate compressor racks in same store?

- Other ideas?

- What cost premium could be tolerated? 1-year payback? 2-year payback?

- What other barriers are there to adoption of advanced case technology?

Industry participation would be vital to any field test/demonstration effort. We don't expect commitments today but would like an indication of willingness to contribute to a demonstration by:

- Hosting test site(s)?

- Providing advanced cases for the field test (gratis or at low cost)?

After about $1.5 \mathrm{hr}$ of discussion around the room, participants were asked to summarize their thoughts to the questions above and turn them in before leaving.

Field test versus lab tests. Of the industry attendees present, 19 offered responses. In addition, three other invited supermarket representatives provided comments before the meeting.

- 14 said field tests of some kind would definitely be needed.

- 8 said laboratory tests would be sufficient.

There was considerable discussion about whether or not the project should proceed to field test at this stage. Many comments from the floor and on the returned questionnaires noted that field trials might best be done by the equipment manufacturers once case laboratory evaluation and design optimization were completed to a satisfactory stage. Numerous comments from the floor expressed a general opinion that the current prototype from this project had not yet reached a satisfactory conclusion, and more work was needed in the lab particularly to optimize the air curtain design. (Twelve persons also mentioned this on the question sheets turned in at the close of the meeting.) It was noted that testing of the prototype and base cases needed to be done at equivalent product temperatures in order to have an "apples to apples" comparison for manufacturers to base their own future development work upon.

Regarding the issue of "how to structure a field test" the responses were evenly split between "side-by-side stores" and single-store approaches. 
Most all of the store representatives and equipment manufacturers indicated willingness to participate and help share the burdens of eventual field testing programs.

\section{Barriers}

- Payback limits - Four of the five store representatives (two at the meeting and three who sent comments beforehand) indicated that two years was the maximum payback they could tolerate for energy efficiency investments. The fifth said up to three years would be okay.

- Other comments - 'product temperature minimums not negotiable'; 'reliability is a must'; 'serviceability/availability of parts and trained service people'; 'should not appear different from what store merchandisers are used to'.

\subsubsection{Summary of Workshop Findings}

Prototype case test results reported at this workshop have shown a significant ( $>25$ percent) reduction in energy consumption compared to the baseline - albeit with slightly higher product temperatures. However, case refrigeration load was essentially unchanged from the baseline because the DAG design in the prototype together with operation at reduced DAG Reynolds number did not achieve the reduction in ambient air infiltration sought. Further testing of the prototype will establish a comparison point with the baseline at similar product temperature levels. Complete details of the prototype case test setup and the final results are discussed in subsection 10.3.

Feedback from the industry participants to the discussion questions posed indicated that some form of field testing in real supermarket conditions would be needed to give supermarket engineers the confidence needed to use the advanced case design. However, considerable opinion was expressed that perhaps this might best be done by the equipment manufacturers in collaboration with supermarket companies after the laboratory phases are complete. Many persons at the meeting expressed that more laboratory development work is needed especially regarding the air curtain. Further research was highly recommended to improve our understanding of air curtain performance and develop more effective means to reduce infiltration loads on open, vertical display cases. We are hopeful of being able to extend the current project for a year or so to address the air curtain and DAG design issues so that the original goals for case load reduction can be approached.

Table 11 lists the attendees of the January 2004 workshop. 
Table 11. Attendees list for the advanced supermarket display case workshop, January 2004

\begin{tabular}{|l|l|}
\hline \multicolumn{1}{|c|}{ Attendee } & \multicolumn{1}{c|}{ Organization } \\
\hline Arun Vohra & DOE \\
Van Baxter & Oak Ridge National laboratory \\
David Walker & Foster-Miller, Inc. \\
Ramin Faramarzi & Southern California Edison \\
Homayun Navaz & Kettering University \\
Len Limbacher & Columbus Showcase \\
John Roche & Hussmann Refrigeration \\
Massoud Neshan & Wolverine Tube Co. \\
Ed Estberg & Raleys Supermarkets \\
Hugh Henderson & CDH Engineering \\
Bob Stone & Wal-Mart \\
Kevin Finlay & Wal-Mart \\
John Murray & Sporlan Valve Co. \\
Neel Gupte & Tyler Refrigeration \\
Dean Swofford & Hill Phoenix Refrigeration \\
Larry Howington & Hill Phoenix Refrigeration \\
Michael Kauk & Barker Company \\
Bill Wehmeier & Emerson Inc. \\
Dennis Welch & Federated Industries \\
Terry Leonard & Southern Store Fixtures \\
Viktor Anderson & Structural Concepts Corp. \\
Jason Williams & Killion Industries \\
Sonya Beckley & Floyd \& Partners \\
Alex St. John & General Electric \\
Robert Weeks & Kysor//Warren Refrigeration \\
Cynthia Gage & US EPA \\
Georgi Kazachki & Hill Phoenix Refrigeration \\
Ed Symbournes & Specialty Fabricators \\
Bruce Colburn & Southern California Edison \\
John Lutton & Southern California Edison \\
Rafik Sarhadian & Southern California Edison \\
Mike Siefkes & Southern California Edison \\
Rod Vickers & Southern California Edison \\
\hline
\end{tabular}




\section{SELECTION OF ENERGY EFFICIENCY MEASURES}

As noted in subsection 4.2 open, multi-deck, medium temperature display cases typically comprise about half of the refrigerated fixtures in a store (2). An independent survey of stores in southern California indicated the same result (Figure 4). In addition, medium temperature fixtures and storage coolers account for roughly 70 to 75 percent of the total store refrigeration load with open, multi-deck cases contributing about $3 / 4$ of that fraction (2). Consequently, the focus of this investigation has tilted toward the open, vertical, multi-deck medium temperature case type.

Past research reveals that infiltration is the biggest component of cooling load in medium temperature open vertical display cases, demonstrated in Figure 5 (ㅇ) . Therefore, in selecting energy efficiency measures, close attention was paid to technologies that can reduce infiltration load.

The selection of the energy efficiency measures (EEMs) in this study was performed based on the following criteria:

- Near-term implementation.

- Potential for energy-efficiency improvements.

- Enhancement of product temperature maintenance.

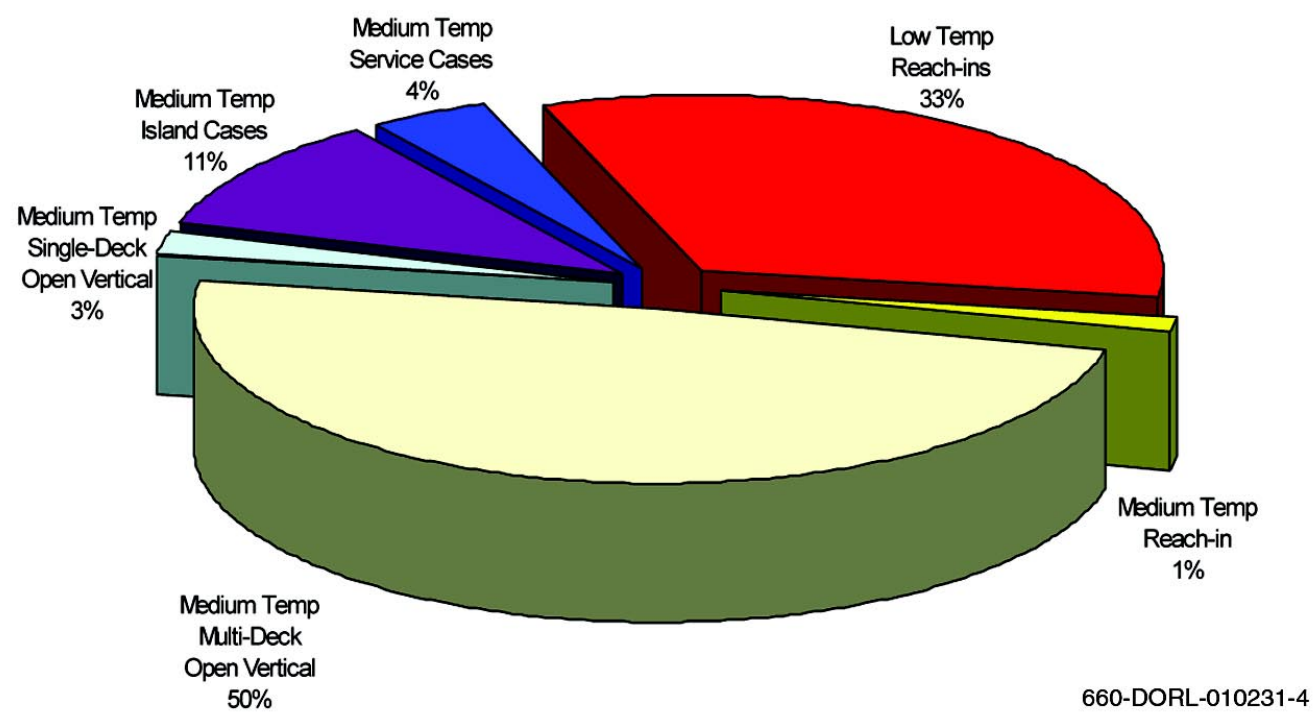

Figure 4. Percentage breakdown of fixture lineal feet by type in a typical supermarket 


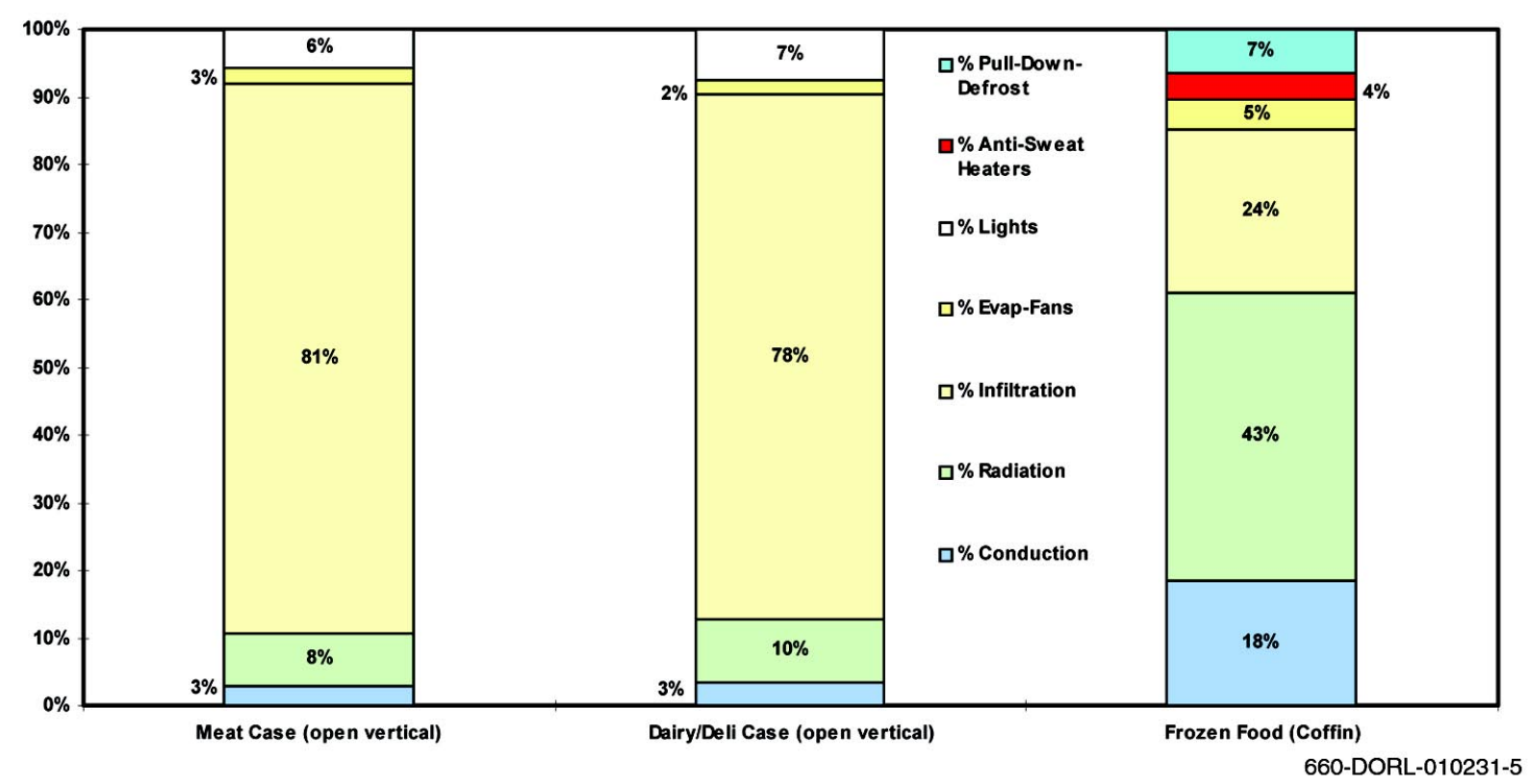

Figure 5. Refrigeration load percentage breakdown for meat, dairy/deli and frozen food fixtures ( $\underline{8})$

The selected EEMs were grouped into five main categories. Technologies within these categories were expected to improve the refrigeration system performance, maintain a tightly targeted product temperature and reduce the electric power usage of the case. Economics of the candidate EEMs can have an influential role in their market adoption. The study, however, focused on their technical feasibility and energy impacts.

\subsection{EEMs Descriptions and Categories}

1. Evaporator coil assembly EEM consisted of a high-efficiency evaporator coil and a dual port thermostatic expansion valve (TXV). The high-efficiency coil features a large heat transfer surface with staggered fin design, and enhanced tubing as shown in Figure 6.

Table 12 compares the key operational characteristics of the high-efficiency and baseline evaporator coils. The dual-port TXV regulates the refrigerant flow by opening its larger port during post-defrost pull down periods and reducing the flow through its smaller port for normal operation. This design was ultimately installed in the prototype case.

2. The air distribution EEM used in the prototype case consisted of two tangential fans (Figure 7) operated by a 1/3 horsepower dual shaft electronically commutated motor (ECM), which has an efficiency of about 80 percent. Each tangential fan has 30 blades 


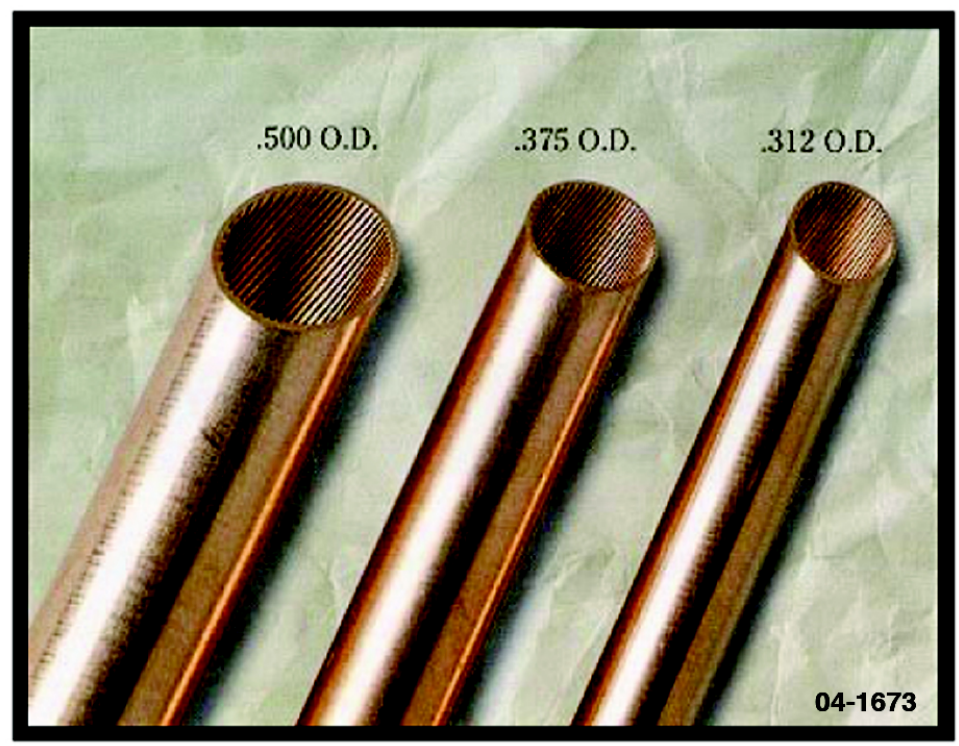

Figure 6. Internally enhanced copper tubing

Table 12. Summary of baseline and prototype case evaporator specifications

\begin{tabular}{|l|l|l|}
\hline \multicolumn{1}{|c|}{ Design Parameter } & \multicolumn{1}{|c|}{ Baseline Evaporator } & \multicolumn{1}{c|}{ Prototype Evaporator } \\
\hline Overall Dimensions & $\begin{array}{l}81 \text { in. W } \times 7-5 / 8 \text { in. } \mathrm{Hx} \\
12 \text { in. D }\end{array}$ & $\begin{array}{l}81 \text { in. W } \times 7-1 / 2 \text { in. } \mathrm{Hx} \\
13-9 / 16 \text { in. D }\end{array}$ \\
\hline Number of Circuits & 4 & 6 \\
\hline Number of Tube Passes per Circuit & 12 & 12 \\
\hline Liquid Line Fed & Front & Back \\
\hline Number of Fins per Inch & 2 & $\begin{array}{l}3 \text { for 5 Front Rows } \\
\text { 4 for 7 Rear Rows }\end{array}$ \\
\hline Fin Material & Flat Aluminum & Flat Aluminum \\
\hline Tube/Pipe Outside Diameter & $9 / 16$ in. & $3 / 8$ in. \\
\hline Tube/Pipe Material & Copper & Copper \\
\hline Tube/Pipe Internal Surface & Smooth & Enhanced \\
\hline
\end{tabular}
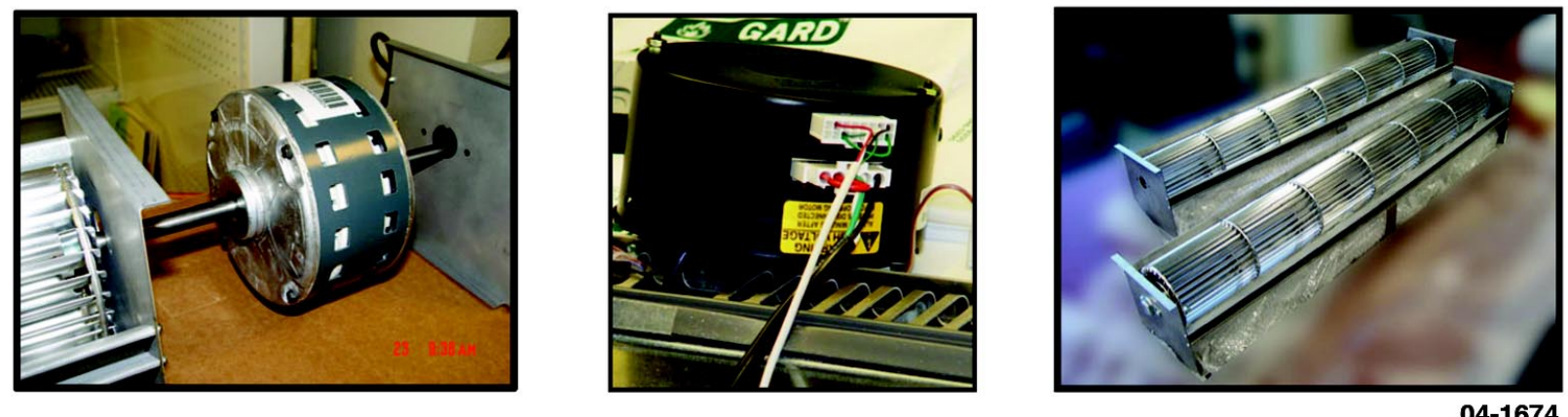

Figure 7. Photographs of the tangential fans and evaporator fan motor 
with an airflow inlet/outlet angle of $180 \mathrm{deg}$. The wheel diameter and length of each tangential fan is 4.5 in. and 36 , in. respectively.

3. A liquid-to-suction heat exchanger (LSHX) EEM made of aluminum and utilizing microchannel heat exchangers was used in the prototype case (Figure 8). In each module, a liquid channel is sandwiched by 12 vapor tubes. These vapor tubes are 5/32 in. in diameter and 38 in. long. The liquid channel dimensions are: $3 / 32$ in. $\mathrm{D}$ x 1.57 in. $\mathrm{W} x$ $40 \mathrm{in.} \mathrm{L.} \mathrm{The} \mathrm{measured} \mathrm{heat} \mathrm{exchanger} \mathrm{effectiveness,} \mathrm{defined} \mathrm{as} \mathrm{the} \mathrm{ratio} \mathrm{of} \mathrm{actual} \mathrm{to}$ maximum possible heat transfer, was about 70 percent.

4. The lighting system EEM use consisted of high-efficiency super T8 fluorescent lamps (30W, 4100K) with low ballast factor electronic ballast (EB) (Figure 9). The power factor for the EB was over 90 percent. The input voltage of the EB was $120 \mathrm{~V}$ with line current of $0.79 \mathrm{~A}$.

5. Air curtain EEM work involved the investigation of the air curtain effectiveness in reducing infiltration load and maintaining the food products at a prescribed temperature. The investigation also identified those parameters that can have significant impact on the reduction of entrained air and infiltration rate. The analysis employed a combination of numerical and experimental methodologies, to ascertain the thermo-fluid characteristics of air curtain and to better understand the entrainment phenomenon. An initial $\left(1^{\text {st }}\right.$ generation) redesign of the discharge air grill (DAG) assembly and a reduced air curtain flow Reynolds number at the DAG were implemented into the prototype case.

Figure 10 depicts the $1^{\text {st }}$ generation new DAG geometry with deflector angle $(\theta)$ of 20 deg. This design concept was developed based on a series of computational fluid dynamics (CFD) modeling efforts as discussed in Section 8.

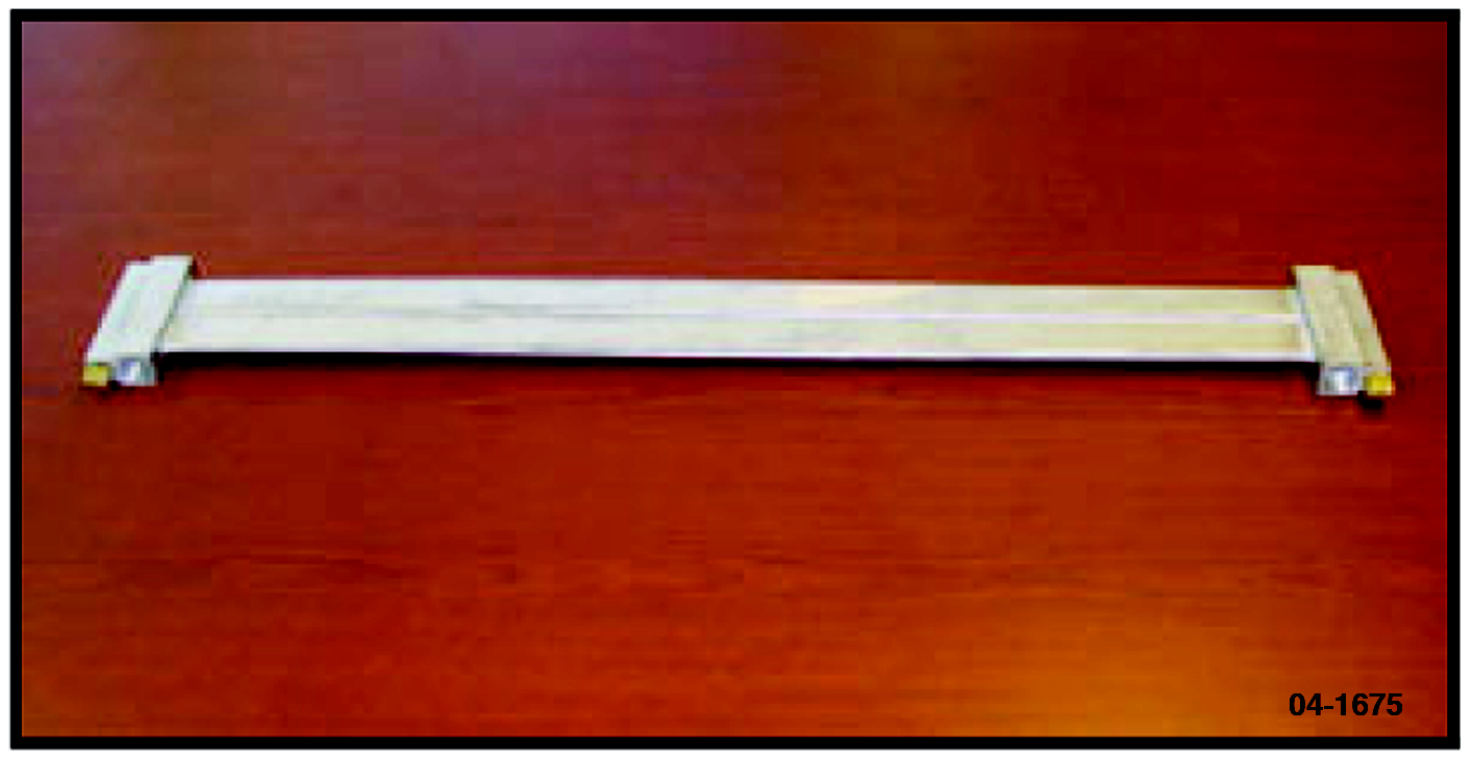

Figure 8. Photographs of the LSHX 

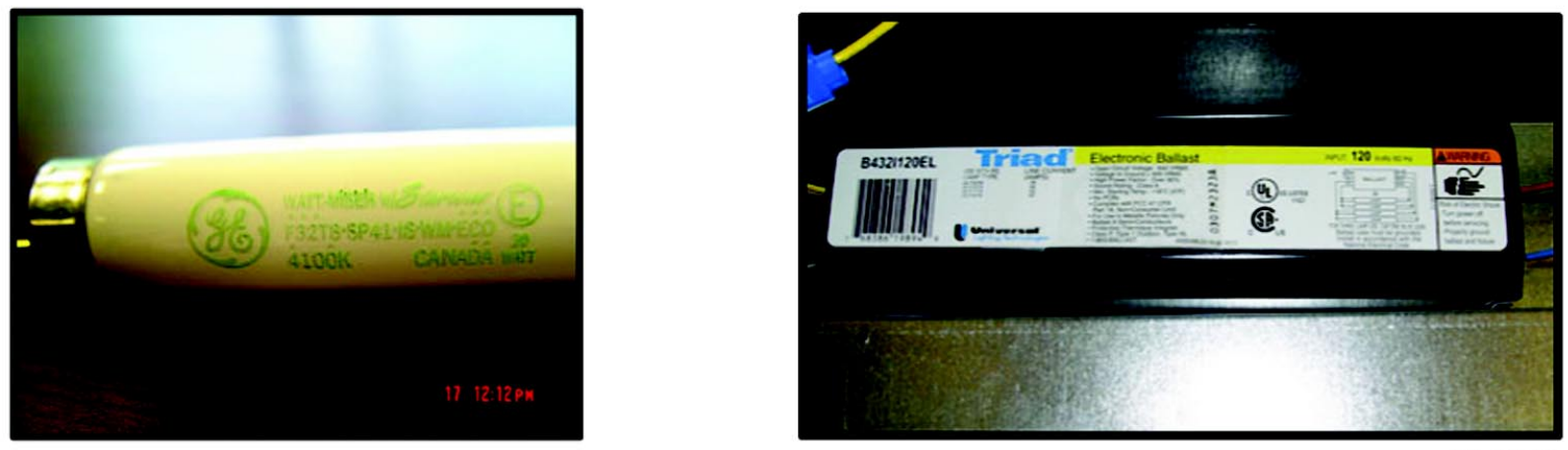

04-1676

Figure 9. Photographs of the lighting system

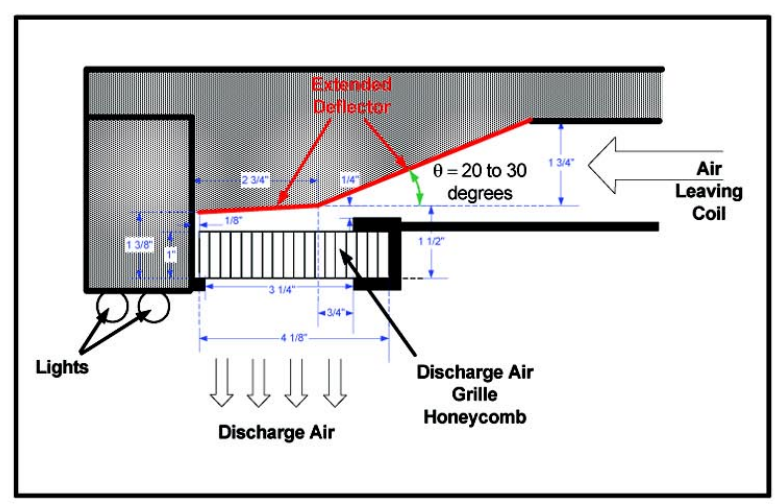

Total Case cfm

750

Flow Control

Constant cfm via VSD

Air Flow at DAG

$210 \mathrm{cfm}$

Back Panel Air Flow

$540 \mathrm{cfm}$

Average DAV**

$100 \mathrm{ft} / \mathrm{min}$

Operational Reynolds No. 3,400

Turbulence Intensity $\quad 2 \%$

Angle of Throw 12 degrees towards inside of the case

Extended Deflector Angle $\theta=20$ degrees

660-DORL-010231-6

Figure 10. $1^{\text {st }}$ generation improved geometry of the DAG for infiltration rate reduction 


\section{INVESTIGATION OF DISPLAY CASE PERFORMANCE}

\subsection{Approach}

The investigation of display case performance falls into two phases. A brief description of each phase is provided below.

In Phase I, the energy impact of a number of viable display case EEMs was quantified by performing whole building hourly simulations. A special version of the U.S. Department of Energy's DOE-2 program (10) was used to develop a model based on actual supermarket information. The model was then calibrated using available end-use monitored data to increase confidence in simulation results. As part of the calibration process, the non-weather dependent electrical loads, such as lighting and equipment as well as their corresponding hourly usage profiles were reconciled with the end-use monitored data. Simulation results were used to identify viable energy-efficient technologies for implementation in the second phase of the project. Additionally, under this phase, a standard eight-foot long, medium temperature fourdeck open vertical display case was selected and evaluated in the laboratory to establish baseline performance data. Finally, a combination of analytical and experimental methods was used to study the air curtain performance. These methods involved the use of digital particle image velocimetry (DPIV) and laser Doppler velocimetry (LDV) to visualize and quantify the air flow field along the plane of air curtain. The DPIV and LDV results were also used to calibrate a CFD model, which was later used to simulate the air curtain performance. Results obtained from CFD modeling were used to determine the best DAG configuration and velocity profile to minimize the infiltration load of the display case. Based on the obtained intelligence, limited modifications were made to the geometry of the baseline case DAG assembly. The thermal performance of the display case was then reevaluated.

In Phase II, findings from previous phases were used to develop energy-efficiency specifications for fabricating a new display case (prototype). The shell of the prototype case was fabricated with a $1^{\text {st }}$ generation redesign of the DAG configuration with the intention to reduce the infiltration load of the case. The next step included the design, fabrication and installation of the rest of the energy-efficient components discussed in Section 5. The thermal performance of the prototype was then evaluated.

\subsection{Test Design and Setup}

\subsubsection{Test Design}

The test setup and design followed guidelines specified in ASHRAE Standard 72-98. The refrigeration system was charged with a hydroflurocarbon refrigerant (R-404A). The 
refrigeration systems controller maintained a fixed saturated condensing temperature (SCT) of $95^{\circ} \mathrm{F}\left( \pm 0.5^{\circ} \mathrm{F}\right)$ for all tests. The display cases were tested in a controlled environment room at Southern California Edison's (SCE) Refrigeration and Thermal Test Center (RTTC).

All tests were performed under steady-state conditions following ASHRAE Standard 72-98. Average discharge air temperature (DAT), which was the critical control point was maintained at $30^{\circ} \mathrm{F}\left( \pm 0.5^{\circ} \mathrm{F}\right)$ to prevent maximum product temperature from exceeding $41^{\circ} \mathrm{F}$. The controlled environment chamber was maintained at a constant dry bulb (DB) temperature of $75.2^{\circ} \mathrm{F} \pm 2^{\circ} \mathrm{F}$ and wet bulb of $64.4^{\circ} \mathrm{F} \pm 2^{\circ} \mathrm{F}$, corresponding to 55 percent relative humidity (RH), throughout the entire $24 \mathrm{hr}$ test period. The intensity of ambient lighting in the controlled environment room was 115 foot-candles and was in compliance with the ASHRAE standard, which requires a minimum of 74.4 foot-candles. The foot-candle measurement was taken at a distance of $1 \mathrm{ft}$ from the air curtain. The entering liquid refrigerant temperature and pressure, measured at 6.1 in. of pipe length from the display case, were maintained at $80^{\circ} \mathrm{F}$ and $214 \mathrm{psig}$ (corresponding to SCT of $\sim 94^{\circ} \mathrm{F}$ ). These parameters meet the ASHRAE standard, which requires the entering liquid temperature be $80.6^{\circ} \mathrm{F} \pm 5^{\circ} \mathrm{F}$, and SCT be maintained between $89.6^{\circ} \mathrm{F}$ and $120.2^{\circ} \mathrm{F}$.

The display case was mounted on special platform to allow installation of a customized condensate pipe/valve arrangement. The piping and valve assembly transferred condensate from the fixture into the container placed on the digital scale. Figure 11 shows the fixture with this custom drainage assembly.

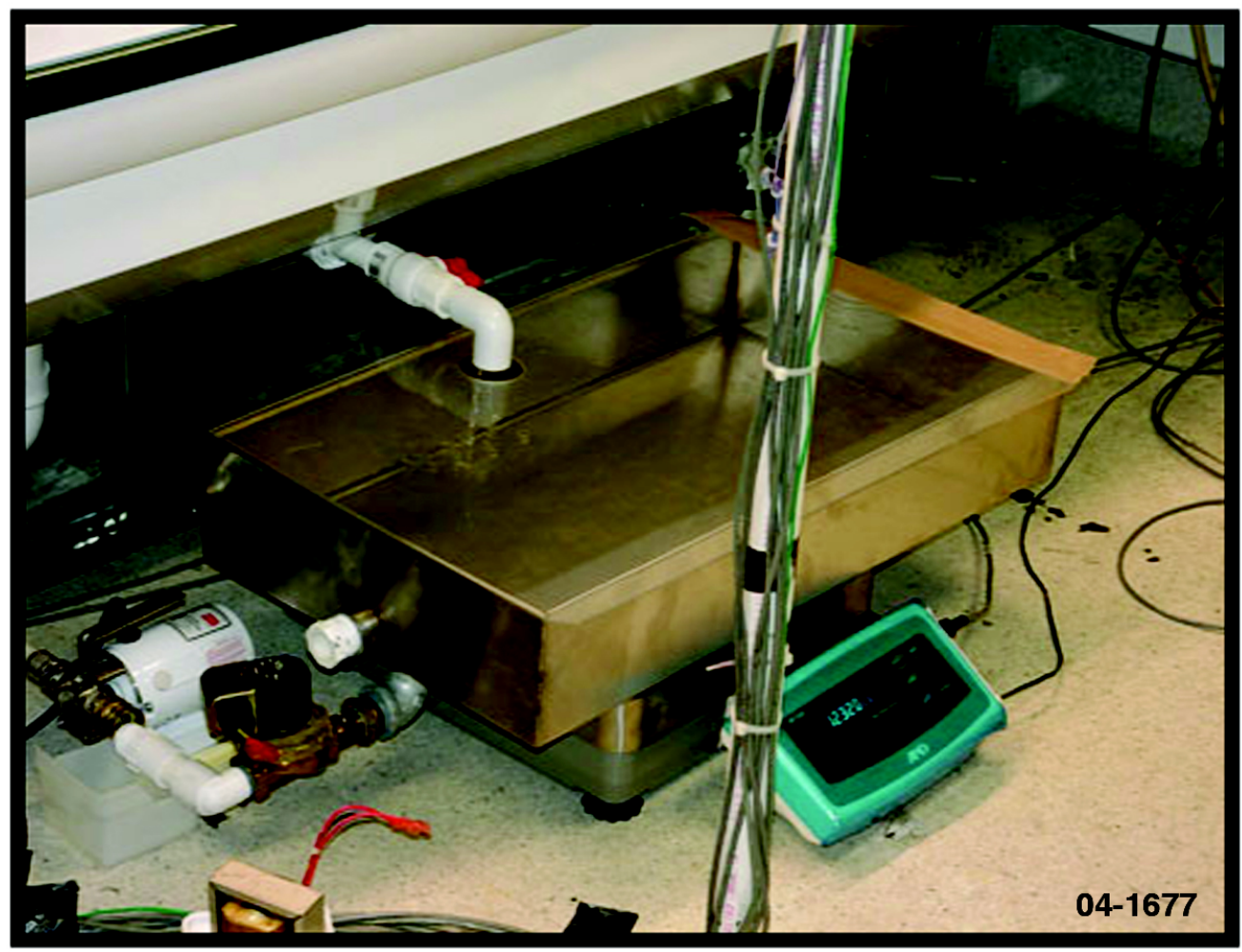

Figure 11. Custom raised frame assembly and special drain piping/valve arrangement 
The product temperatures were monitored at 24 locations within the display case (Figure 12). For each display shelf, two product simulators were located at the left end, the right end and the center. At each left, right, center locations, one product simulator was placed on the shelf surface at the front of the shelf and one at the rear edge of the shelf. As a result, six product simulators were used for each of four shelves.
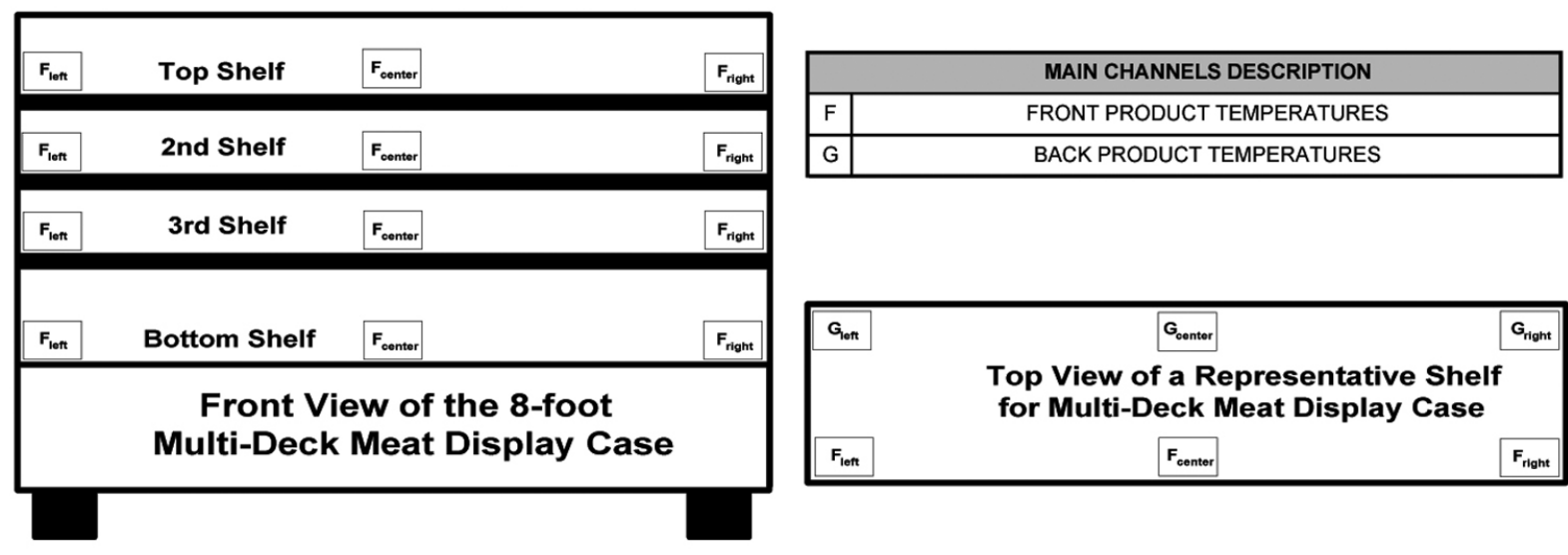

660-DORL-010231-7

Figure 12. Location of product simulators inside the display case

ASHRAE 72-98 also requires food product zones be filled with test packages and dummy products to simulate the presence of food product in the cases (Figure 13). According to ASHRAE standard, food products are comprised of 80 percent to 90 percent water, fibrous materials, and salt. Therefore, plastic containers completely filled with a sponge material soaked in a 50 percent \pm 2 percent by volume solution of propylene glycol and distilled water were used to simulate the product during the tests. The spaces in the test display case where temperature measurement was not required were stocked with dummy products to stabilize the temperature in the case and account for transient heat transfer effects.
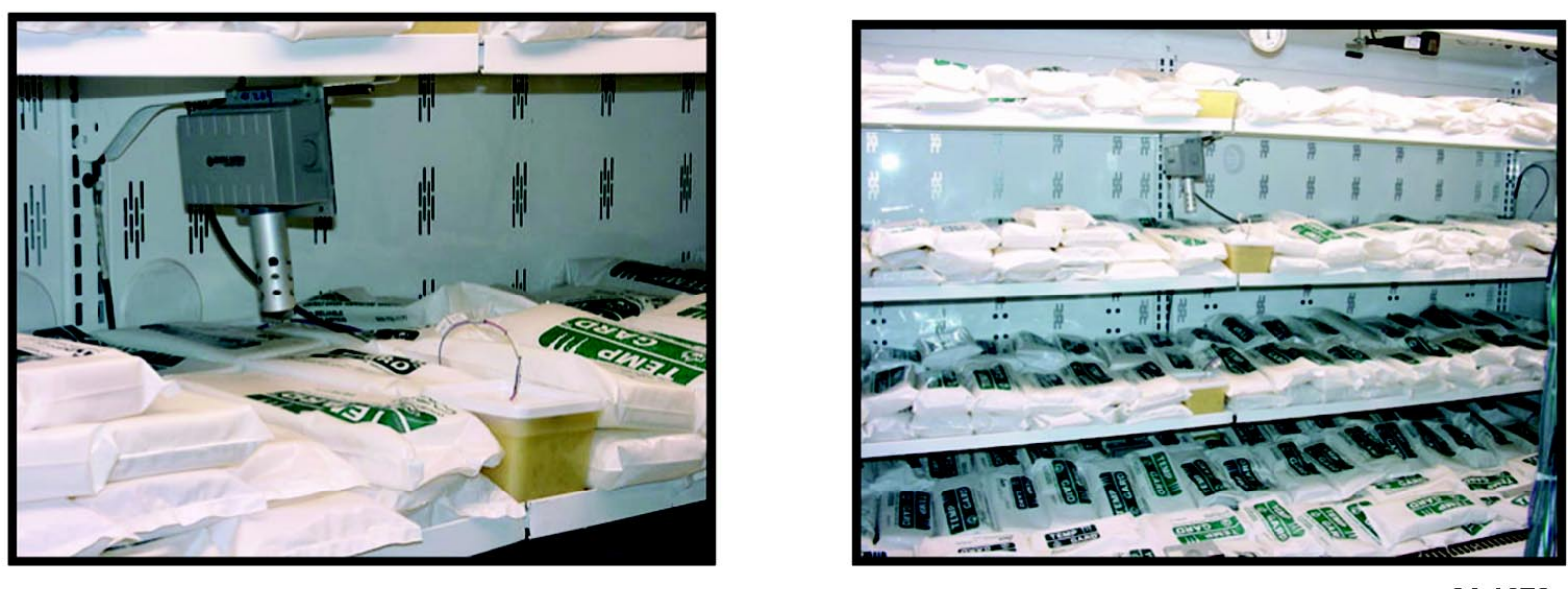

04-1678

Figure 13. Simulated and dummy product used in the display case 
The test was designed with a strong emphasis on proper equipment setup, instrumentation, and data acquisition of the test scenarios. Results obtained from all tests addressed the following key parameters.

- Compressor power and energy, (kW, kWh).

- Total system power and energy (less condenser), (kW, kWh).

- Evaporator fan motors power and energy (kW, kWh).

- Refrigeration energy, (Btu).

- Case total cooling load, (Btu/hr).

- Case latent and sensible load, (Btu/hr).

- Condensate quantity, (lb/hr, lb).

- $\quad$ Product temperatures, $\left({ }^{\circ} \mathrm{F}\right)$.

\subsubsection{Test Scenarios}

The following three tests were performed under this project.

1. Baseline Test.

2. Air Curtain Sensitivity Tests.

3. Prototype Test.

\subsubsection{Instrumentation}

Prior to the tests, all temperature and pressure instruments were calibrated. With the objective of minimizing instrument error and maintaining a high level of repeatability and accuracy in the data, careful attention was paid to the design of the monitoring system. The monitoring plan was developed based on the following guidelines:

- Use of sensors with the highest accuracy available.

- Minimization of sensor drift errors by use of redundant sensors.

- Utilization of calibration standard instruments of the highest accuracy.

- Elimination of interference from power conductors and high frequency signals by doubleshielding sensor leads.

The instrumentation system has special grade type-T thermocouples accurate to $\pm 0.1^{\circ} \mathrm{C}$, precision $100 \Omega$ platinum resistance temperature device (RTD) inputs accurate to $\pm 0.01{ }^{\circ} \mathrm{C}$, analog inputs from pressure transducers, dew point sensors, flow meters, and CT-transducers. A RS-232 communication link sent one data report that included instantaneous values of all data points every $10 \mathrm{sec}$. Table 13 provides the specifications of the various sensors used in the RTTC's refrigeration system for this test. Figure 14 details the location of sensors within the test fixture. 
Table 13. Specifications of sensors used

\begin{tabular}{|c|c|c|}
\hline Sensor Type & Make/Model & $\begin{array}{c}\text { Accuracy } \\
\text { [NIST Traceable] }\end{array}$ \\
\hline Dew Point & EG\&G DewTrak Model 200 & $\pm 1^{\circ} \mathrm{F}$ \\
\hline Dew Point & $\begin{array}{l}\text { EDGETECH Model } 2000 \text { Dew } \\
\text { Prime DF Dew Point } \\
\text { Hygrometer - S2 Sensor }\end{array}$ & $\pm 0.2^{\circ} \mathrm{C}\left(+0.36^{\circ} \mathrm{F}\right)$ \\
\hline Refrigerant Mass Flow & Micro Motion Model DS065S & $\pm 0.2 \%$ \\
\hline Power & $\begin{array}{l}\text { Ohio Semitronics Model } \\
\text { PC5-062BX680 }\end{array}$ & $\pm 0.5 \%$ F.S. $(.04 \mathrm{~kW})$ \\
\hline Power & Ohio Semitronics Model P-143B & $\pm 1.0 \%$ F.S. $(.08 \mathrm{~kW})$ \\
\hline Pressure & $\begin{array}{l}\text { Setra Transducers } \\
\text { Model } 207 \text { - } 100 \text { and } 500 \text { PSIG } \\
\text { Pressure Ranges }\end{array}$ & $\pm 0.13 \%$ \\
\hline Pressure (differential) & $\begin{array}{l}\text { MAMAC System } \\
\text { Model PR-274/275 }\end{array}$ & $\pm 1.0 \%$ F.S. \\
\hline Temperature (RTD) & $\begin{array}{l}\text { Hy-Cal Engineering } \\
\text { Model RTS-37-A-100 }\end{array}$ & $\pm 0.01 \mathrm{C}$ \\
\hline Temperature (TC) & $\begin{array}{l}\text { Kaye Instruments T/W } 50 \text { through } \\
80 ; \text { Melt No. } 8032\end{array}$ & $\pm 0.1^{\circ} \mathrm{C}$ \\
\hline Velometer & EDRA6 Vane Velometer & $\begin{array}{l} \pm 1.0 \% \text { F.S. } 4 \text { in. head } \\
\pm 2.0 \% \text { F.S. } 1.4 \text { in. head }\end{array}$ \\
\hline Velometer & $\begin{array}{l}\text { TSI Inc. Model No. } 8455 \text { Air } \\
\text { Velocity Transducer }\end{array}$ & $\pm 2.0 \%$ F.S. \\
\hline Humidity & $\begin{array}{l}\text { General Eastern } \\
\text { MRHT2-2-V-D } \\
\text { MRHT2-2-V-O/A } \\
\text { General Eastern Humiscan }\end{array}$ & $\begin{array}{l} \pm 2 \% \\
\pm 1.0 \% \mathrm{RH} \text { (for } 0.5 \text { to } 90 \% \mathrm{RH} \text { range) }\end{array}$ \\
\hline Scale & HP-30K & $+0.1 \mathrm{~g}$ \\
\hline
\end{tabular}

The RTTC's data acquisition system was set up to scan 128 data channels in $10 \mathrm{sec}$ intervals and to log channel outputs in 2 min intervals. Collected data was screened closely to ensure the key control parameters were within acceptable ranges. In the event that any of the control parameters fell outside acceptable limits, the problem was flagged and a series of diagnostic investigations were carried out. Corrections were then made and tests were repeated as necessary. Once the data passed the initial screening process, it was imported to RTTC's customized refrigeration analysis model where detailed calculations were performed. The collected data points from the 2 min intervals were averaged into hourly values where necessary and used for a secondary screening of the results.

Figure 15 shows the location of sensors for measuring air and refrigerant properties at the inlet and outlet of the coil. The air temperature, relative humidity, velocity, and dew point are measured at various locations at the inlet and outlet of the evaporator coil. Figure 15 also shows that the baseline case's evaporator coil consisted of four circuits. For each circuit, the refrigerant temperature was measured at the inlet, mid section, and outlet of the evaporator coil. 


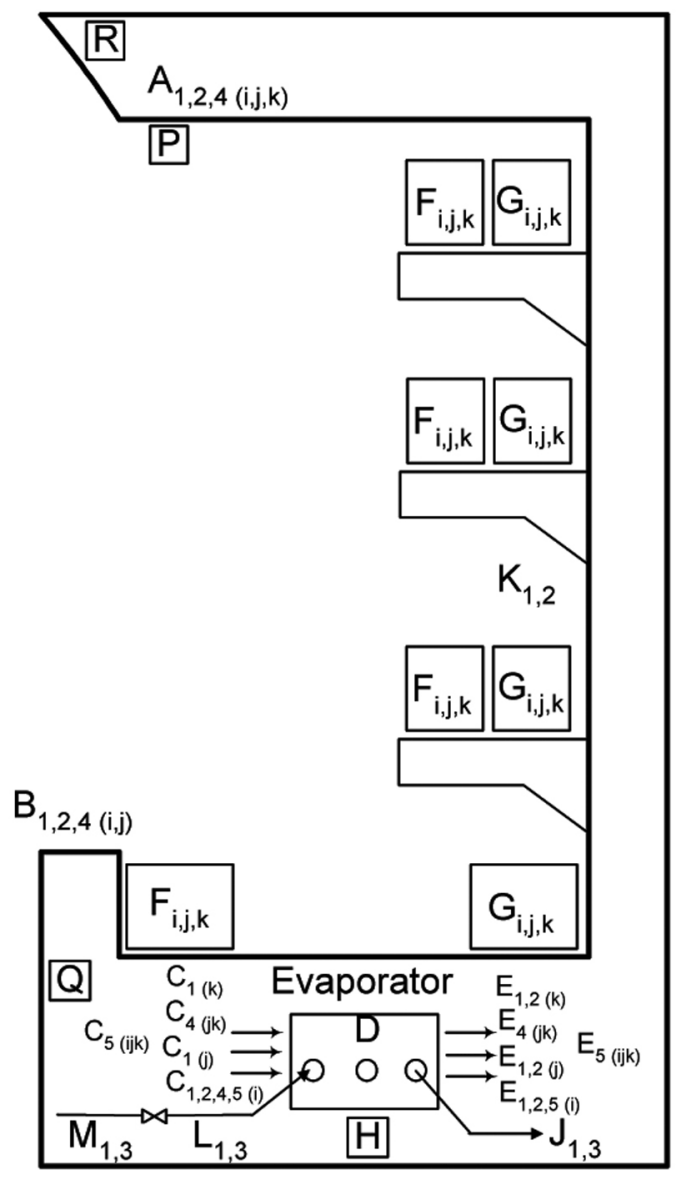

\begin{tabular}{|c|c|}
\hline \multicolumn{2}{|c|}{ MAIN CHANNELS DESCRIPTION } \\
\hline A & DISCHARGE AIR \\
\hline B & RETURN AIR \\
\hline C & AIR ENTERING EVAPORATOR [After Fans] \\
\hline D & COIL SURFACE TEMPERATURE (PER CIRCUIT) \\
\hline E & AIR LEAVING EVAPORATOR \\
\hline F & FRONT PRODUCT TEMPERATURES \\
\hline G & BACK PRODUCT TEMPERATURES \\
\hline H & AIR PRESSURE DIFFERENTIAL ACROSS EVAPORATOR \\
\hline J & REFRIGERANT AT COIL EXIT \\
\hline K & AIR INSIDE CASE CAVITY \\
\hline L & REFRIGERANT AT EXPANSION VALVE EXIT \\
\hline M & REFRIGERANT AT EXPANSION VALVE INLET \\
\hline P & CASE LIGHT - POWER \\
\hline Q & CASE FAN MOTORS - POWER \\
\hline R & ANTI-SWEAT HEATERS - POWER \\
\hline & SUBSCRIPTS (nUmericaI) \\
\hline 1 & TEMPERATURE \\
\hline 2 & RELATIVE HUMIDITY \\
\hline 3 & PRESSURE \\
\hline 4 & VELOCITY \\
\hline 5 & DEWPOINT TEMPERATURE \\
\hline $\mathrm{i}$ & SUBSCRIPTS (roman) \\
\hline $\mathrm{i}$ & LEFT \\
\hline $\mathrm{j}$ & MIDDLE \\
\hline $\mathrm{k}$ & COMBINATION OF RIGHT, MIDDLE, AND LEFT \\
\hline & \\
\hline $\mathrm{j}$ & \\
\hline
\end{tabular}

660-DORL-020231-8

Figure 14. Locations of sensors for open vertical multi-deck meat display case

\subsubsection{Data Acquisition}

A Kaye Instruments Digi-4 Model No. X1520S data scanner was used to log the test data. Kaye's Digi-4 has a special emphasis on temperature measurement, with excellent thermocouple signal processing. The data scanner was set up to process 128 data channels in $10 \mathrm{sec}$ intervals. The scanner was calibrated at the factory, and is traceable to the National Institute of Standards and Technology's (NIST's) standards. As part of the RTTC's quality control protocol, the data acquisition system for the project was designed to be completely independent of the supervisory control computer. This approach was taken to ensure that the data collection would have not been compromised by the control sequence's priority over data acquisition.

Every $10 \mathrm{sec}$, the data acquisition system sampled the scanned data and created time-stamped 2 min averages. The 2 min data was then saved to a file, which was closed at the end of each $24 \mathrm{hr}$ period. Edison engineers reviewed the initial data on site at the RTTC to ensure that the key control parameters were within acceptable ranges. In the event that any of the control parameters fell outside acceptable limits, the problem was flagged. In such cases, test runs were repeated until correction of the problem. Once the data passed the initial screening process, it was downloaded remotely to SCE's Irwindale office for further screening and processing. 

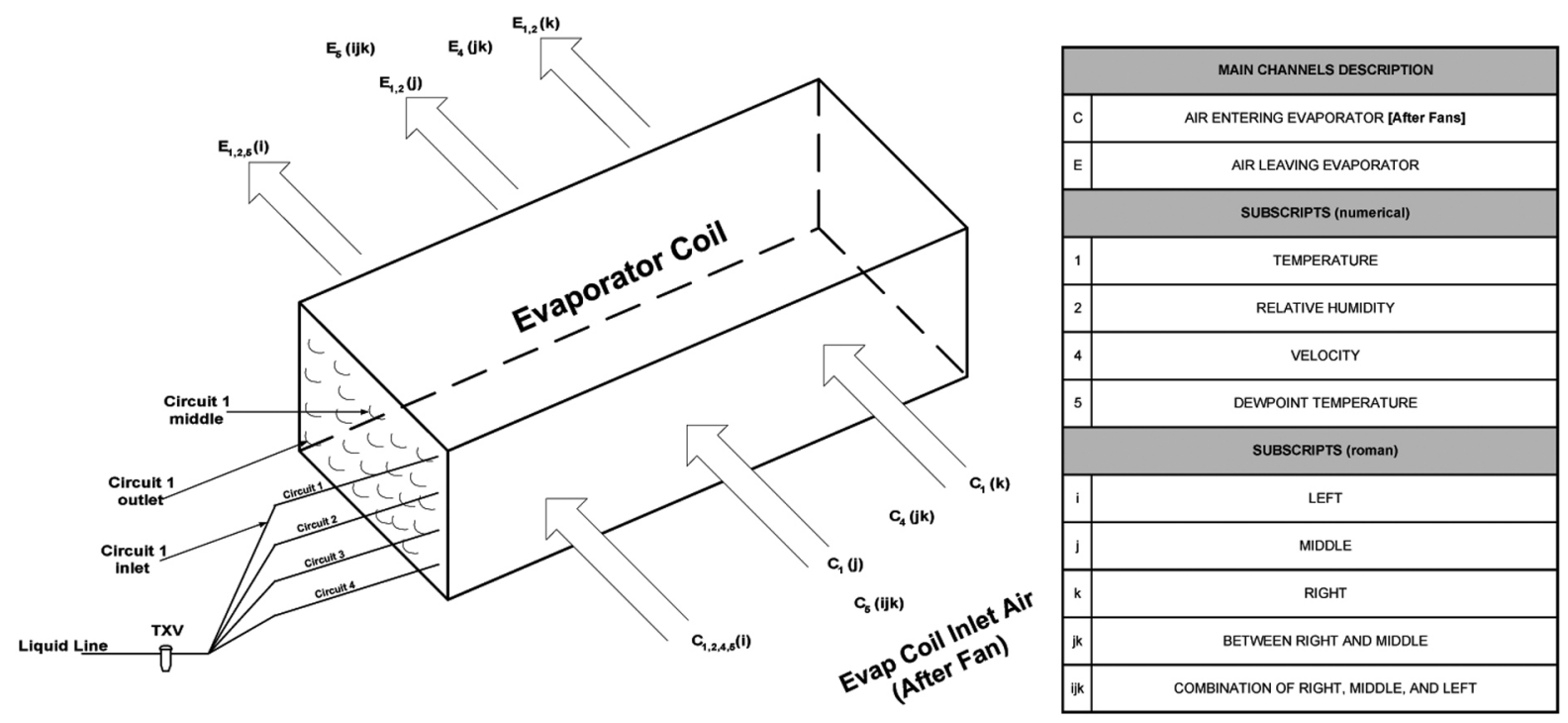

660-DORL-010231-9

Figure 15. Locations of sensors for evaporator coil of the display case

The weight of condensate during each test scenario was measured by using a high precision digital scale with $\pm 0.1 \mathrm{~g}$ accuracy (Figure 16). The data acquisition system received the exact condensate weight measurements from the digital scale every 2 min. Therefore, it became possible to closely monitor and distinguish between the moisture removal from the air during refrigeration cycle and defrost periods were tracked. At the end of each test period, the condensate data was also aggregated into hourly and daily values.

\subsubsection{Controlled Environment Room Condition}

The display case was tested in the controlled environment room of the RTTC. The controlled environment room is an isolated thermal zone served by independent cooling, heating and humidification systems. This allows simulation of various indoor conditions of a supermarket. The sensible cooling load representing people and other heat gain sources is provided by a constant volume direct expansion system reclaiming the waste refrigeration heat via a six-row coil. Auxiliary electric heaters located down-stream of the heat reclaim coils provide additional heating when required. While the air is conditioned to a desired thermostatic set point, an advanced ultrasonic humidification unit introduces precise amounts of moisture to the air surrounding the display cases, representing the latent load due to outside air and people. Figure 17 shows a schematic diagram of the air-conditioning and heating system of the RTTC's controlled environment.

There are three laminar diffusers in the room, each supplying air at approximately 370 cubicfeet-per-minute $(\mathrm{cfm})$. The intensity of ambient lighting in the controlled environment room, as 


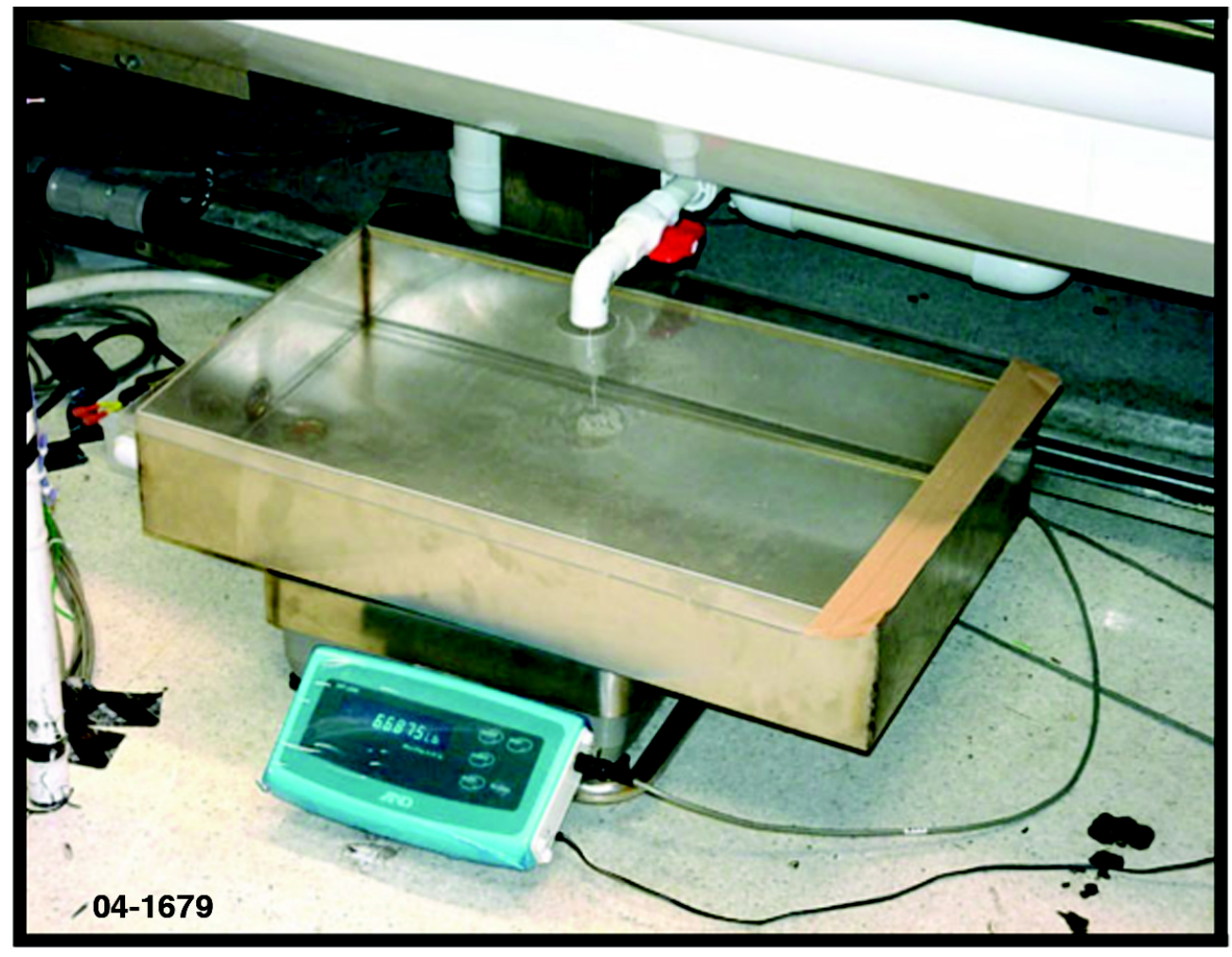

Figure 16. High precision digital scale used to measure the weight of condensate collected

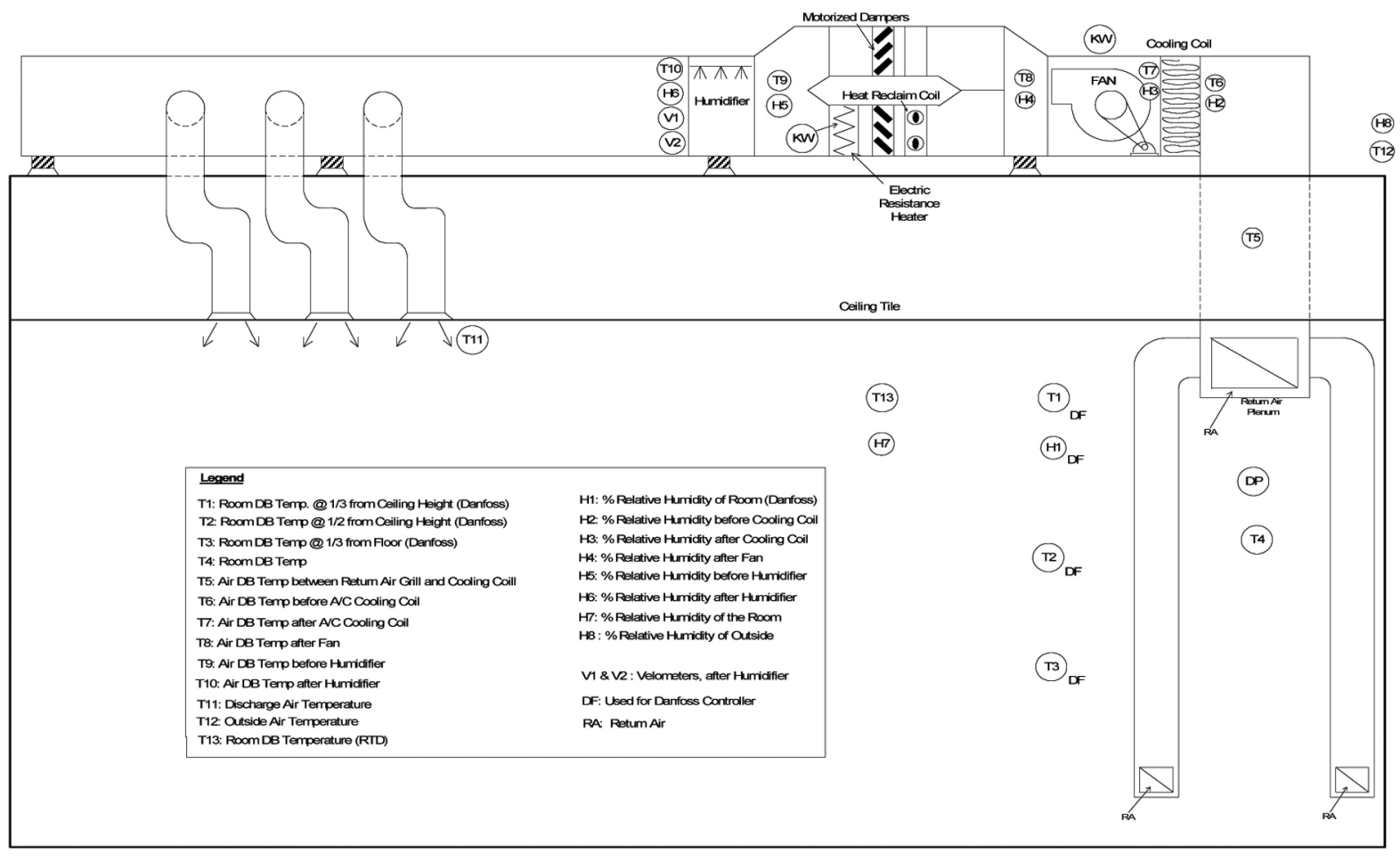

660-DORL-010231-10

Figure 17. Schematic diagram of the air-conditioning and heating system of the RTTC's controlled environment room 
measured from the center of the test fixture opening at a distance of $1 \mathrm{ft}$ from the air curtain, is 115 foot-candles. This meets ASHRAE Standard 72-98, which requires the lighting intensity not be less than 75 foot-candles at this location.

\subsubsection{Baseline Display Case}

The $8 \mathrm{ft}$ long baseline display case with the rated cooling capacity of 1,561 Btu/hr per foot (Figure 18) was equipped with four shelves, three shaded pole fan motors, and two rows of standard T8 canopy lights with electronic ballast. Off-cycle defrost was initiated three times per day and terminated at coil outlet temperature of $\sim 47^{\circ} \mathrm{F}$. Following the manufacturer's published data, the baseline display case was set up to operate at a saturated evaporating temperature (SET) of $17^{\circ} \mathrm{F}$ and discharge air temperature (DAT) of $30^{\circ} \mathrm{F}$.

\subsubsection{Prototype Display Case}

Based on the Phase I findings, a new display case design specification was developed. Hill Phoenix fabricated the shell of the prototype display case with the $1^{\text {st }}$ generation redesign of the air curtain DAG based on the new specification. The RTTC in conjunction with its partners including Wolverine, General Electric and University of Illinois designed, procured, and in some instances fabricated the following technologies:

- Tangential evaporator fans.

- Double-shafted electronically commuted evaporator motor with variable speed control.

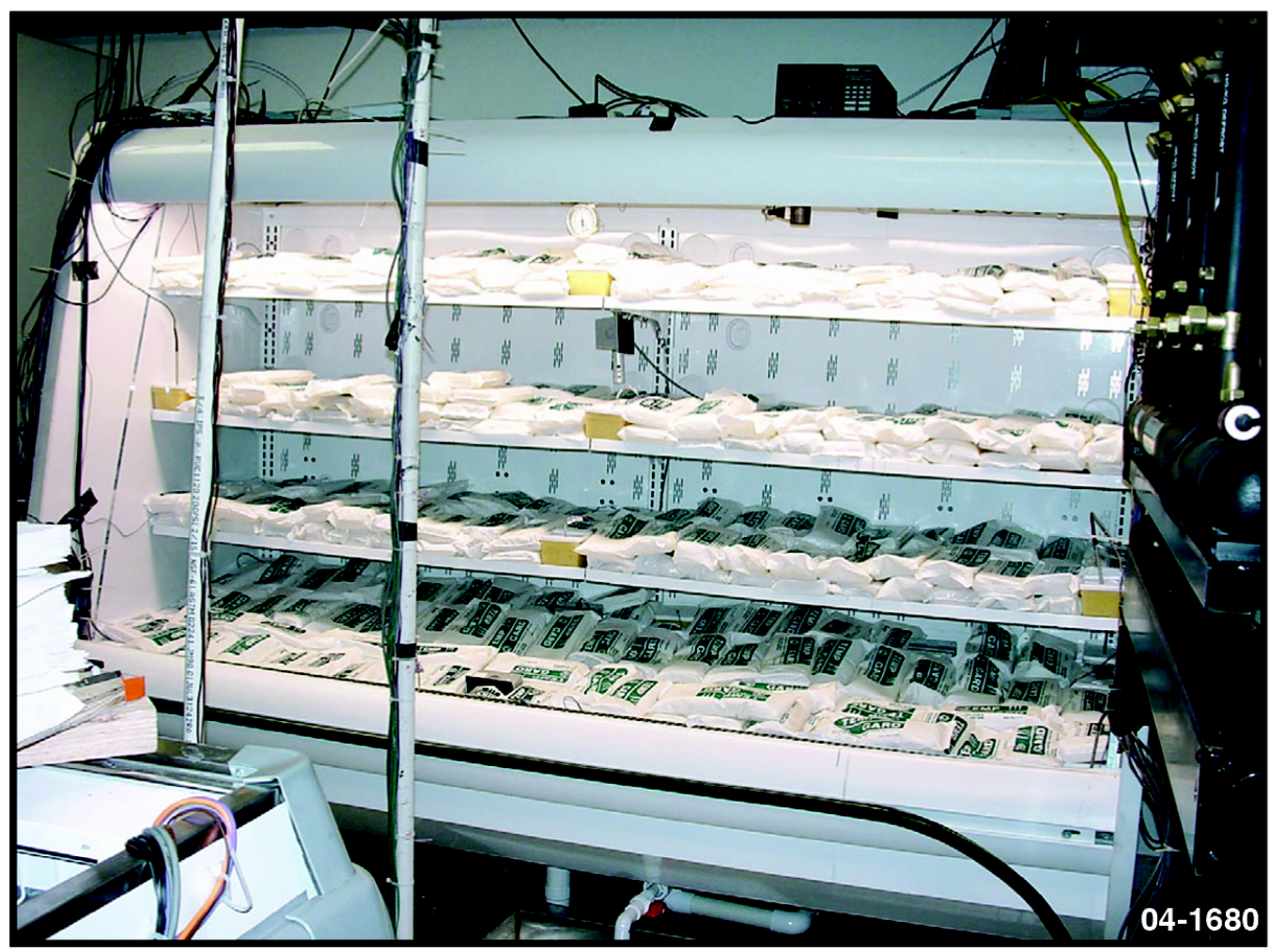

Figure 18. Baseline display case 
- Staggered fin design evaporator with enhanced tubing.

- Micro-channel liquid-to-suction heat exchanger.

- Dual-port thermostatic expansion valve.

\subsubsection{Air Curtain Evaluation}

The main focus of air curtain study was to investigate the effectiveness of the air curtain in maintaining the display case and food products at a prescribed temperature, and identify those parameters that can have significant impact on the reduction of entrained air. It combined several numerical and experimental tools to analyze the air curtain entrainment rate and its minimization.

Digital particle image velocimetry (DPIV) was used to map the two-dimensional flow field in the display case, especially the air curtain and opening areas, and then these flow field results were integrated along the opening to find the entrained air volumetric flow rate. Furthermore, the turbulence kinetic energy and its intensity were mapped at the discharge air grill (DAG) and air curtain by the DPIV method. The laser Doppler velocimetry (LDV) method, measures the vertical component of the velocity. The vertical component of velocity was also employed to determine the mean and fluctuating velocity fields. The LDV test outcome was compared to that of the DPIV. Additionally, a calibrated CFD simulation tool was utilized to perform parametric studies to supplement the experimental tasks. A more detailed discussion of the air curtain analyses is provided in Section 8. 


\section{ENERGY IMPACT ANALYSIS}

\subsection{A Brief Description of the DOE-2.3 Simulation Program}

The DOE-2.3 is an hourly building energy simulation computer program that is widely used by architects, engineers, utility personnel, and other building professionals. It uses an actual weather data to simulate energy systems within a building. The refrigeration algorithms used in this version of the program are component-based, so that a user can build up a system out of individual components. For example, a supermarket system may consist of numerous display fixtures (dairy, produce, frozen food, etc.), attached to various refrigeration circuits (10). These circuits in turn attach to one or more suction-groups, which in turn are built up from one or more compressors. These suction groups discharge into one or more condensers that condense refrigerant vapor, and return liquid refrigerant back to the fixtures. The refrigeration module allows each of these components to be specified individually, as many as required, and connected together to create a system. The simulation algorithms are based on refrigerant mass flow.

The mass flow method decouples the compressor pumping capacity (mass capacity) from the conditions of the refrigerant in the system. This method allows more involved and precise modeling of the various components comprising a refrigeration system (compressors, condensers, subcoolers, fixtures, etc.). In addition, this approach is easier to understand and implement.

\subsection{Component-Based Model}

The refrigeration simulation model is component-based. Each major device, such as a refrigerant loop, fixture, compressor, condenser, etc. is specified separately, and the model simulates each component separately using a dedicated algorithm.

To simulate a refrigeration system(s), the user first defines one or more refrigerant loops. Then each component, such as a dairy case or compressor, is defined and attached to the appropriate loop. The control parameters, such as the compressor sequencing, suction pressure control, condenser temperature control method, and usage profiles are also specified by the user. The program uses this information, together with the information required to simulate the building, HVAC systems, and weather data, to derive the hourly load profiles and energy consumption of the mechanical systems.

Because each device exists as a separate component, it is straightforward to create a library of the various types of equipment that you may want to study. The program incorporates this 
library concept, and a wide variety of makes and model numbers of various components are available, including display fixtures, compressors and compressor racks, condensing units, subcoolers, and refrigerants. For each component, the library stores all the information necessary to simulate the device.

A supermarket commonly contains on the order of 40 to 50 display fixtures, several refrigerant loops, a variety of compressors or compressor racks, and one or more condensers. Using the pre-defined components in the library substantially reduces the time required to create a model of a refrigeration system, and also reduces the number of errors that could otherwise be generated.

The component-based structure exists within the program as well. Each component, such as a display fixture, contains information on the simulation algorithm it requires in the hourly simulation. The program calls these various algorithms as required. This modularity makes the algorithms more understandable, and also makes it easier to add additional component algorithms for in the future.

\subsection{Refrigeration System Components}

The model incorporates algorithms for all of the common components in a supermarket refrigeration system.

- Display Fixtures - Selections include low temperature single deck, low temperature multi-deck, low and medium temperature glass door, medium temperature single deck, medium temperature multi-deck, service cases, and specialty cases. Specifications include the make and model (for fixtures in the library), case length, discharge air temperature, saturated-suction temperature, defrost type and control, lighting options, fan options, liquid-suction interchangers, and anti-condensate controls.

- Walk-ins and Prep Areas - Selections include storage walk-ins, walk-in boxes with glass doors (e.g., dairy, beverage and floral boxes), preparation areas that may be fully enclosed or have one side open to the sales area, and other perimeter zones that are air conditioned from the refrigeration system (e.g., bakery prep areas, pharmacy, etc.). Specifications include the make and model (for components in the library), size, temperature, location, reach-in doors, walk-in doors, refrigeration load, lighting, evaporator coils, defrost type and control, fans, and internal loads.

- Branch Circuits - The program defines a branch circuit as the piping that connects a fixture to its suction group on the low side, and its condensing unit/subcooler on the high side. Specifications for branch circuits include the piping location and either the piping size, length and U-value, or the design pressure drop and temperature change.

- Suction Groups - Each display fixture, walk-in, etc. connects via piping to a single compressor or group of compressors that operate at a common suction pressure, defined as a "suction group." Specifications for the compressors include the make and model (for 
components in the library), type and number, and performance data of compressors, suction pressure set point and control method, variable-speed options, and desuperheating method. Compressor types in the library include a variety of reed and Discus semihermetic, screw, scroll, and open-drive compressors.

- Refrigerant Loops - Each suction group discharges to a "high side" header that in turn is attached to (one or more) condensing units. This high side header is the common point for all fixtures, compressors, condensers, and other components that comprise a refrigeration system, and is the basis for the definition of a refrigerant loop. Different suction groups may discharge to the same or different refrigerant loops. Specifications for the loop include the refrigerant type, and condensing temperature set point and control. Instead of discharging into a condensing unit, a refrigeration loop can discharge into another refrigeration loop. For example, a $-25^{\circ} \mathrm{F}$ suction group serving an ice cream tub may discharge into another refrigeration loop into a served by $\mathrm{a}+10^{\circ} \mathrm{F}$ suction group.

- Condensers/Heat Rejection - Selections include both air-cooled condensers and evaporative condensers. Specifications include the size, design criteria, fan/pump power, and capacity control mechanism, including variable-speed. In addition to condensers, a refrigeration loop may reject its heat through a water-cooled condenser to a water circulation loop. These loops in turn are served by chillers, cooling towers, fluid coolers, ground-coupled heat exchangers, rivers, etc.

- Subcooolers - Selections include both mechanical and ambient subcoolers.

\subsection{Description of the Modeled Supermarket Site}

An actual single story $51,000 \mathrm{ft}^{2}$ supermarket site with aspect ratio of 1.5 was selected for this study (Figure 19). The store is located in Temecula, CA.

The following describes envelope and system characteristics of this supermarket. The information below was obtained from the actual as-built construction documents.

\subsubsection{Envelop Description}

- Roof materials include 3 in. of expanded polystyrene and 6 in. of $140 \mathrm{lb}$, heavy weight, and dried concrete.

- Exterior wall material include 8 in., $100 \mathrm{lb}$ concrete block filled with $120 \mathrm{lb}$ solid grout.

- Floor was comprised of 12 in. soil and 4 in., $140 \mathrm{lb}$, heavy weight, dried concrete as well as $3 / 8$ in. clay tile pave. 


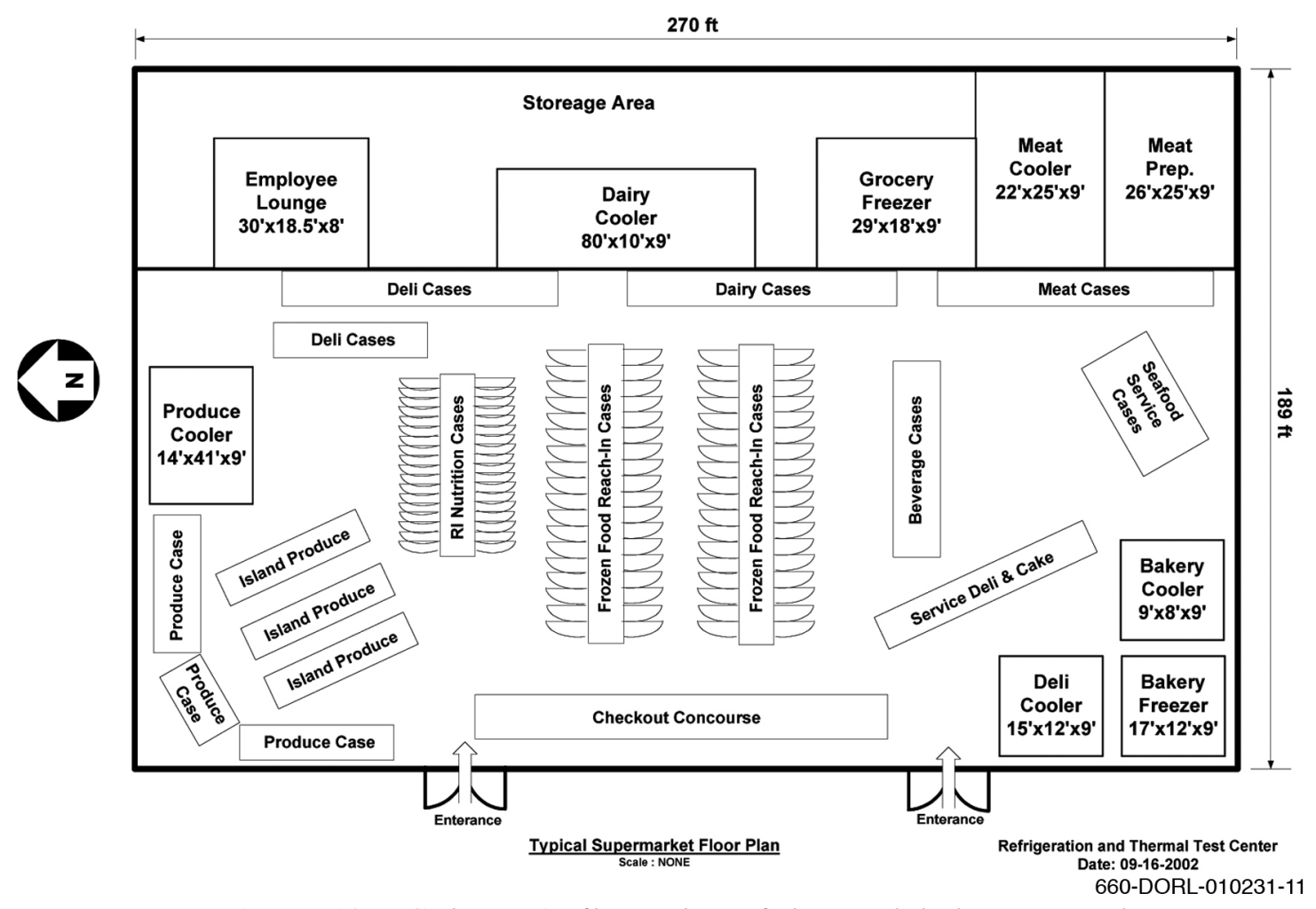

Figure 19. Schematic floor plan of the modeled supermarket

\subsubsection{Lighting and Day Lighting Systems}

The sales area light fixtures were equipped with FO32T833K lamps and electronic ballasts. The lighting intensity in sales area was $1.714 \mathrm{~W} / \mathrm{ft}^{2}$. In addition, there were $30,4 \mathrm{x} 8 \mathrm{ft}$ skylights. Natural daylight through the skylights allowed for reduction of sales lighting, utilizing a dimming control.

\subsubsection{HVAC System}

A constant volume packaged roof top unit provided the HVAC needs of the sales area. A Variable Speed Drive (VSD) modulated the speed of the supply air fan according to sales area's thermostatic needs. The 700,000 Btu/hr cooling capacity of the unit was provided by two, 6 cylinder semi-hermetic compressors with 25 and $35 \mathrm{hp}$ using R-507. Both compressors operated at a suction temperature of $40^{\circ} \mathrm{F}$ and condensing temperature of $100^{\circ} \mathrm{F}$. The HVAC system shared the condenser of the refrigeration system to reject its heat. The supply fan circulated a maximum of $29,000 \mathrm{ft}^{3}$ of air per minute. The fan motor was rated at $25 \mathrm{hp}$ and equipped with VSD. The system introduced $6,300 \mathrm{cfm}$ of outside air to meet ventilation requirements in the sales area. 


\subsubsection{Refrigeration System}

A multiplex compressor system consisting of four different suction groups provided refrigeration for display cases, prep rooms and walk-ins. The following describes the operational characteristics of each suction group.

Suction Group $1-$ Low Temp $\left(-25^{\circ} \mathrm{F}\right)$ :

- The common design temperature for this suction group was $-25^{\circ} \mathrm{F}$ with a design condensing temperature of $85^{\circ} \mathrm{F}$. This suction group was comprised of three discus compressors with 22,15 and $7.5 \mathrm{hp}$, which were capable of providing a total of 208,200 Btu/hr of cooling using R-507. This suction group served a line up of ice cream reach-ins with 80 doors, a line up of frozen food reach-ins with 35 doors, a 5 door reachin frozen bakery, and a four door reach-in nutrition fixture. It also, served an $18 \mathrm{ft}$ x $29 \mathrm{ft}$ $\mathrm{x} 9 \mathrm{ft}$ grocery freezer and a $12 \mathrm{ft} \times 17 \mathrm{ft} \times 9 \mathrm{ft}$ bakery freezer.

Suction Group $2-$ Medium Temp $\left(+15^{\circ} \mathrm{F}\right)$ :

- The common design suction temperature for this loop was $+15^{\circ} \mathrm{F}$ with a design condensing temperature of $90^{\circ} \mathrm{F}$. This suction group was comprised of three discus compressors with 20,15 and $7.5 \mathrm{hp}$, which were capable of providing a total of $352,600 \mathrm{Btu} / \mathrm{hr}$ of cooling using R-507. This suction group serves $108 \mathrm{ft}$ of open multideck deli fixtures, $72 \mathrm{ft}$ of open multi-deck meat fixtures, $42 \mathrm{ft}$ of service fixtures, and $20 \mathrm{ft}$ of sandwich fixtures. Also, this suction group serves a produce cooler $(14 \mathrm{ft} \times 41 \mathrm{ft}$ x $9 \mathrm{ft}$ ), a bakery cooler ( $8 \mathrm{ft} \times 9 \mathrm{ft} \times 9 \mathrm{ft})$, and a deli cooler (12 ft x $15 \mathrm{ft} \times 9 \mathrm{ft})$.

Suction Group $3-$ Medium Temp $\left(+20^{\circ} \mathrm{F}\right)$ :

- The common design suction temperature for this loop was $+20^{\circ} \mathrm{F}$ with a design condensing temperature of $90^{\circ} \mathrm{F}$. This suction group was comprised of four discus compressors with $25,10,10$ and $7.5 \mathrm{hp}$, which were capable of providing a total of $509,900 \mathrm{Btu} / \mathrm{hr}$ of cooling using R-507. This suction group served $80 \mathrm{ft}$ of open multideck dairy fixtures, $104 \mathrm{ft}$ of open multi-deck produce fixtures, $72 \mathrm{ft}$ of island produce fixtures, $18 \mathrm{ft}$ of open multi-deck floral station, $104 \mathrm{ft}$ of open multi-deck beverage fixtures, a 3-door reach-in floral case, and a 2-door reach-in nutrition case. Also, this suction group served a dairy cooler ( $10 \mathrm{ft} \times 80 \mathrm{ft} \times 9 \mathrm{ft})$, and a meat cooler ( $22 \mathrm{ft}$ x $25 \mathrm{ft} \times$ $9 \mathrm{ft})$.

Suction Group $4-$ Medium Temp $\left(+35^{\circ} \mathrm{F}\right)$ :

- The common design suction temperature for this suction group was $+35^{\circ} \mathrm{F}$ with a design condensing temperature of $90^{\circ} \mathrm{F}$. This suction group was comprised of two discus compressors with $25,10 \mathrm{hp}$, which were capable of providing a total of $352,600 \mathrm{Btu} / \mathrm{hr}$ of cooling using R-507. This suction group served the meat preparation area $(25 \mathrm{ft} x 26 \mathrm{ft} x$ $9 \mathrm{ft}$ ) as well as offices and employee break room. In addition, it provided a total 
subcooling of $331,000 \mathrm{Btu} / \mathrm{hr}$ to suction groups 1,2 , and 3. This suction group does not serve any fixture line ups.

\subsubsection{Condenser}

A single multi-circuited evaporative condenser with 152 tube circuits served the HVAC and refrigeration systems. The condenser's rated capacity was 5,880,000 Btu/hr with volumetric airflow rate of $61,000 \mathrm{cfm}$.

\subsection{Modeling Approach}

Prior to performing energy simulations, it was imperative to increase the confidence in forecasting capability of the model. Typically, calibration of the computer model with end-use monitored data, coupled with realistic weather data is necessary. Six months of hourly weather data collected at one of SCE's weather stations, closest to the subject site was obtained and converted into a required DOE-2.3 format. Under a separate project short-term end-use monitoring had been conducted at this particular site providing field data for calibration of the DOE-2.3 model. The cumulative energy consumption of various end uses captured in the monitoring data from June through November 1998 is shown in Figure 20. After the model was calibrated, full-year parametric simulations were performed at six different locations.

The monthly energy use of the refrigeration and HVAC compressors at the site is shown in Figure 21. As expected, the highest peaks took place during summer period.

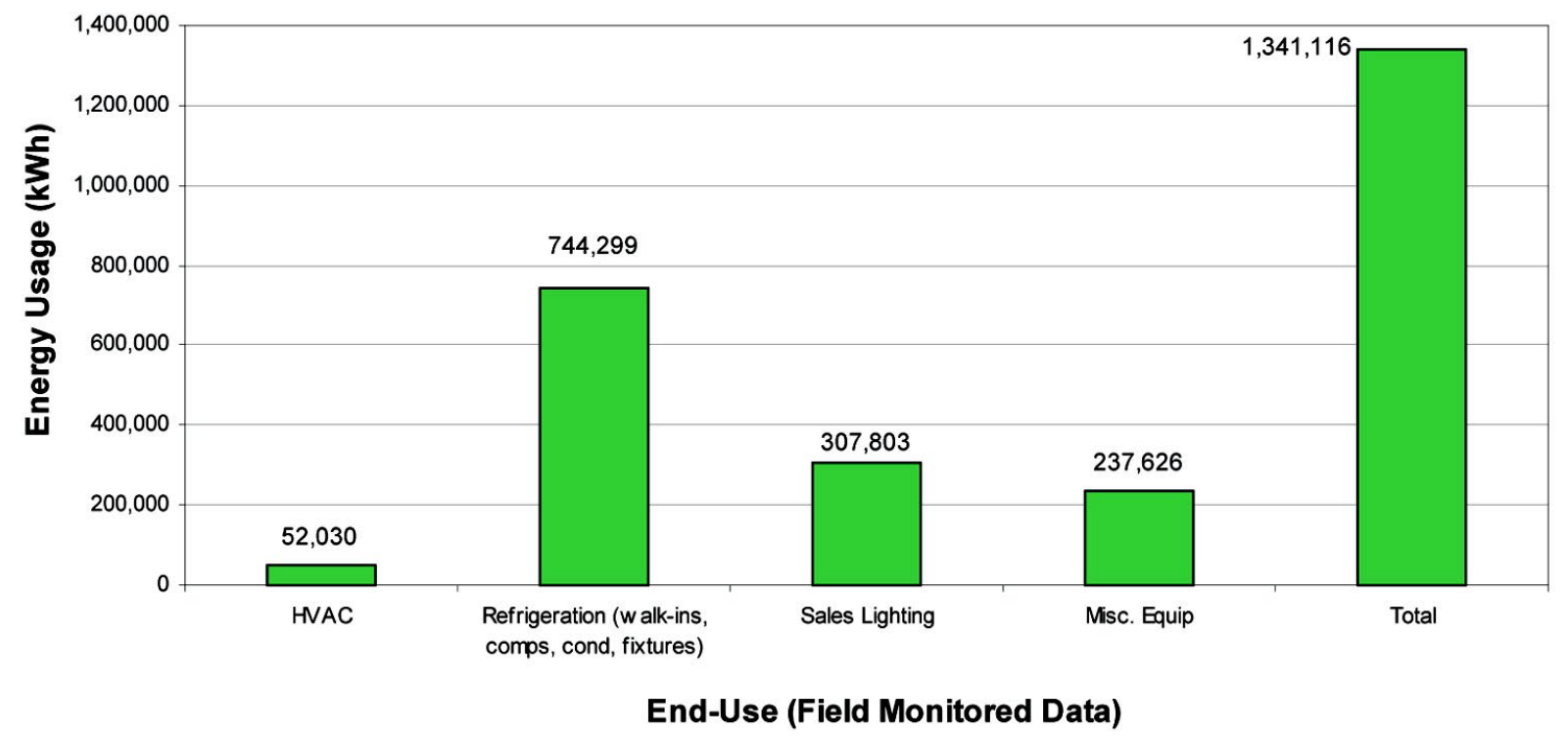

660-DORL-010231-12

Figure 20. Energy usage by end-use (six months field monitored data) 


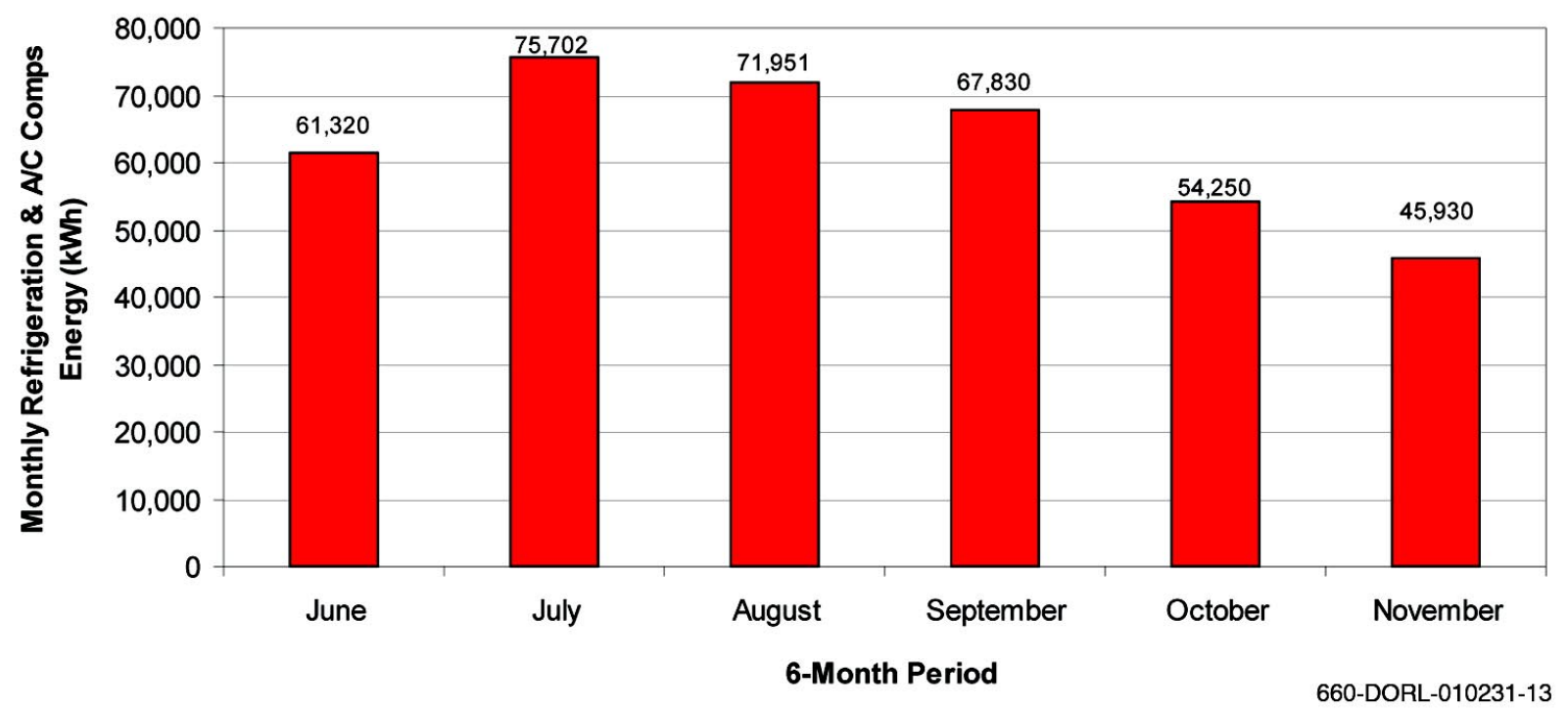

Figure 21. Monthly refrigeration and HVAC compressors energy usage (field monitored data)

During the process of DOE-2.3 model calibration, the non-HVAC inputs such as lighting and miscellaneous loads were adjusted to match those of the field monitored data. After matching the peak $\mathrm{kW}$ usage of these two end-uses, their schedules were modified to yield a reasonably close energy usage profiles. Figure 22 compares the calibrated DOE-2.3 results with field data. Simulation results seem to be in close agreement with field monitored data.

\subsection{Simulation Results}

\subsubsection{Base Case Simulation}

The following energy efficiency measures (EEMs) were simulated and their impact on the refrigeration or cooling load and energy usage of the facility were analyzed based on Los Angeles weather data. (Not all of the simulated EEMs were evaluated during the case testing portions of this project.)

1. Modular coil and defrost.

2. High efficiency evaporator, LSHX and advanced controls.

3. ECM for evaporator fan.

4. Tangential fan with ECM motor and VSD controller.

5. Improved air curtain.

6. Glass doors on open vertical medium temperature cases.

7. T8EB w/ enhanced interior film. 


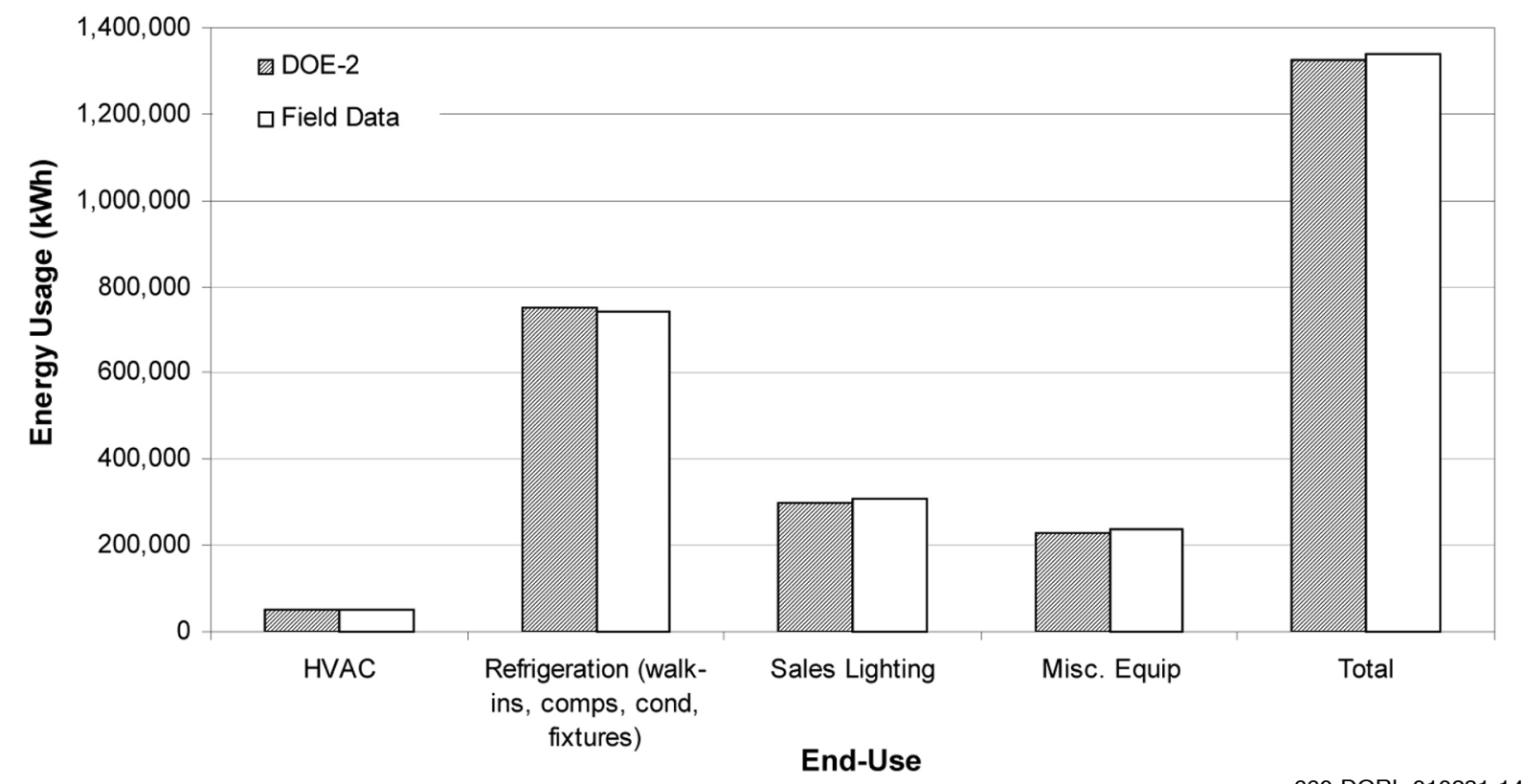

Figure 22. Comparison of energy usage by end-use

8. Combination of $1,2,3,4,5,7$.

9. Combination of $1,2,3,4,6,7$.

Figure 23 indicates that measures that reduced the infiltration to the open vertical medium temperature cases had the greatest impact in reducing cooling (refrigeration) loads. The simulation showed that the combinations ( 8 and 9 ) lowered the site cooling or refrigeration load from $2,676 \mathrm{kBtu} / \mathrm{hr}$ to either 2,259 or $1,882 \mathrm{kBtu} / \mathrm{hr}$ (or 16 percent to 30 percent).

Similarly, EEMs with most influence on infiltration to open vertical cases had the highest impact on the refrigeration energy use (Figure 24). The annual refrigeration energy use of the site is estimated to drop from 1,518,517 $\mathrm{kWh}$ to anywhere from $1,299,379$ to $1,100,769 \mathrm{kWh}$ (or 14 percent to 28 percent).

Implementation of the simulated EEMs is estimated to lower the total annual energy usage at the Los Angeles site by a maximum of 14 percent (Figure 25).

\subsubsection{Simulation for Six Different Locations}

In addition to the base case scenario, the EEMs 8 and 9 were simulated and their impact on the cooling load of the facility was analyzed based on six different locations in nation. Figure 26 depicts the WB and DB temperatures for all six locations. As depicted, Houston and Chicago had the highest and lowest temperatures, respectively. 


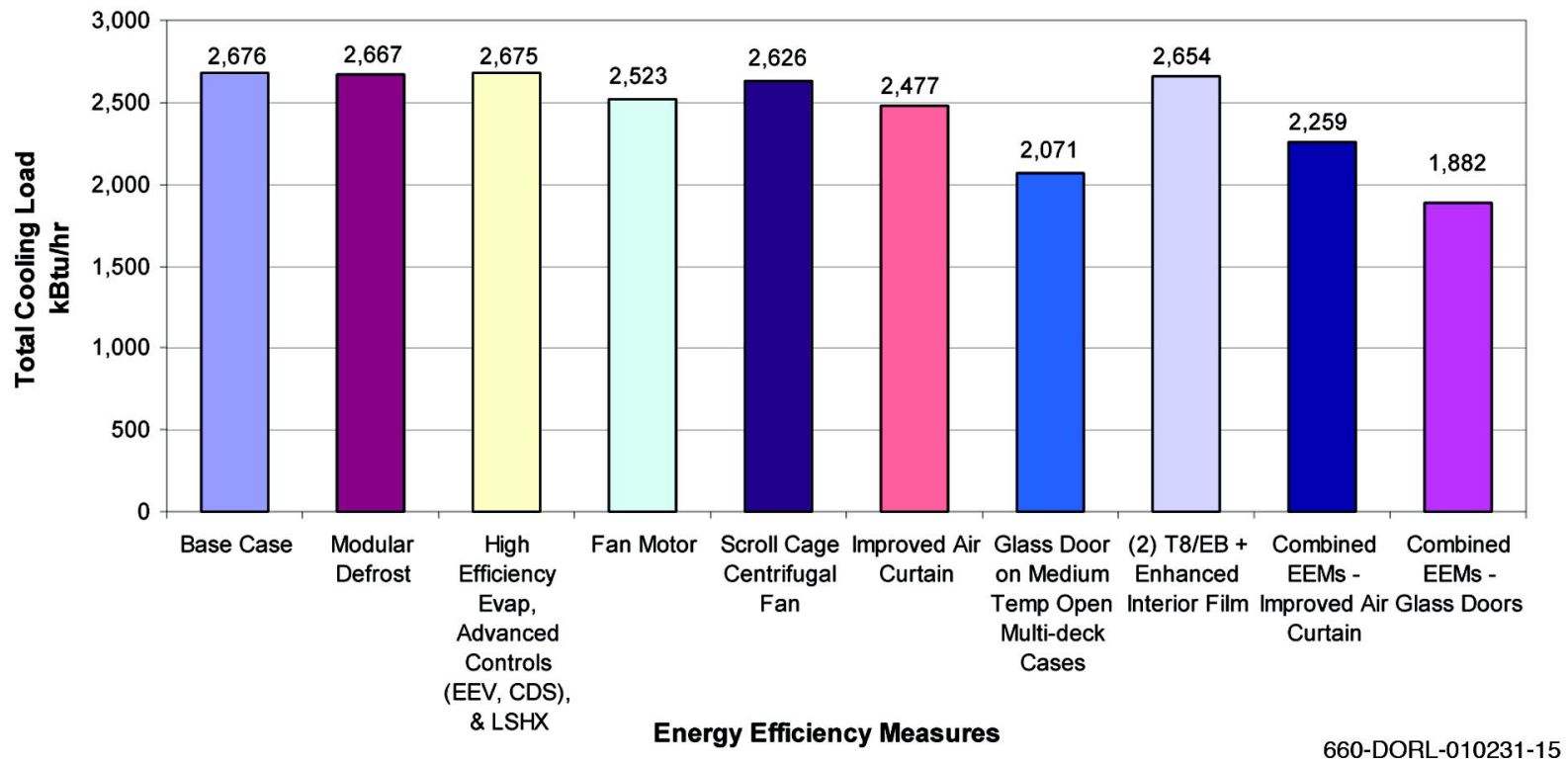

Figure 23. Impact of energy efficiency measures on total cooling load

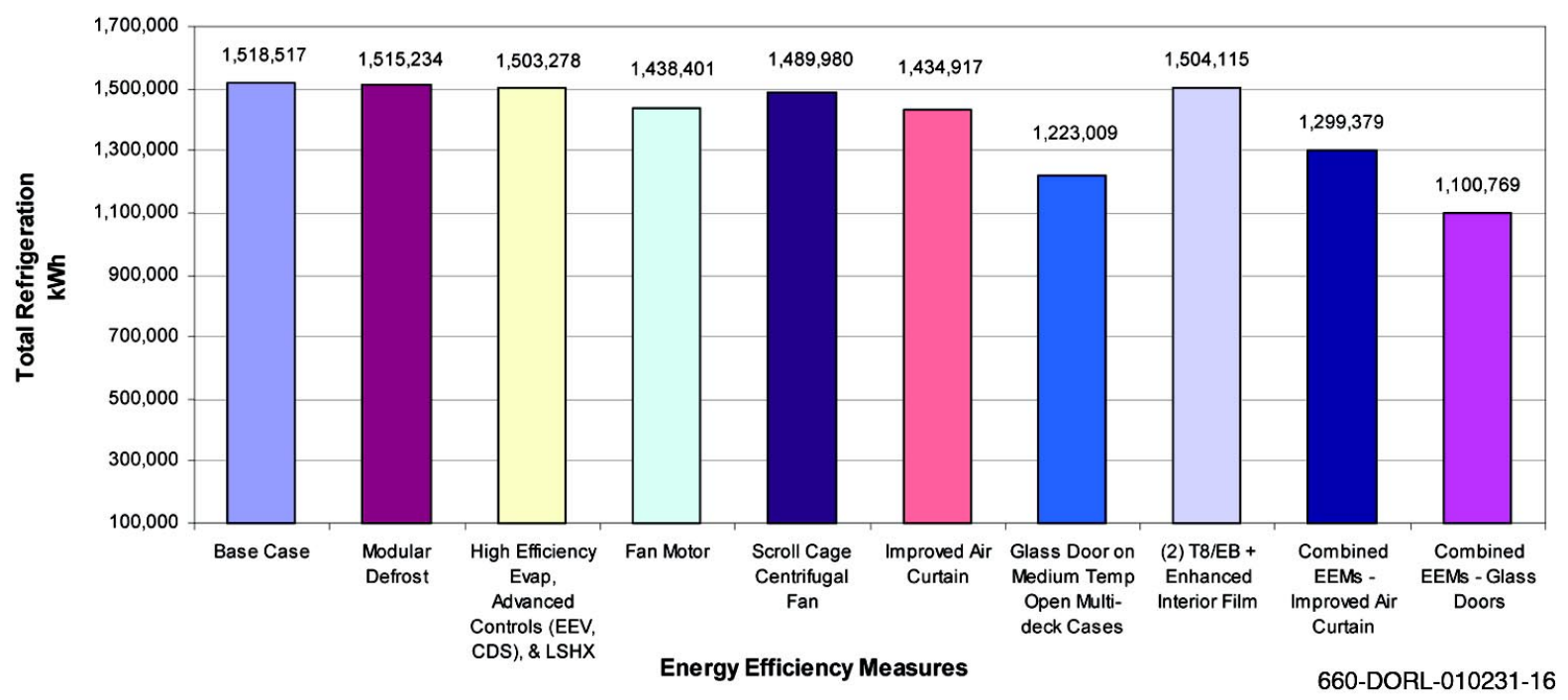

Figure 24. Impact of energy efficiency measures on total refrigeration energy 


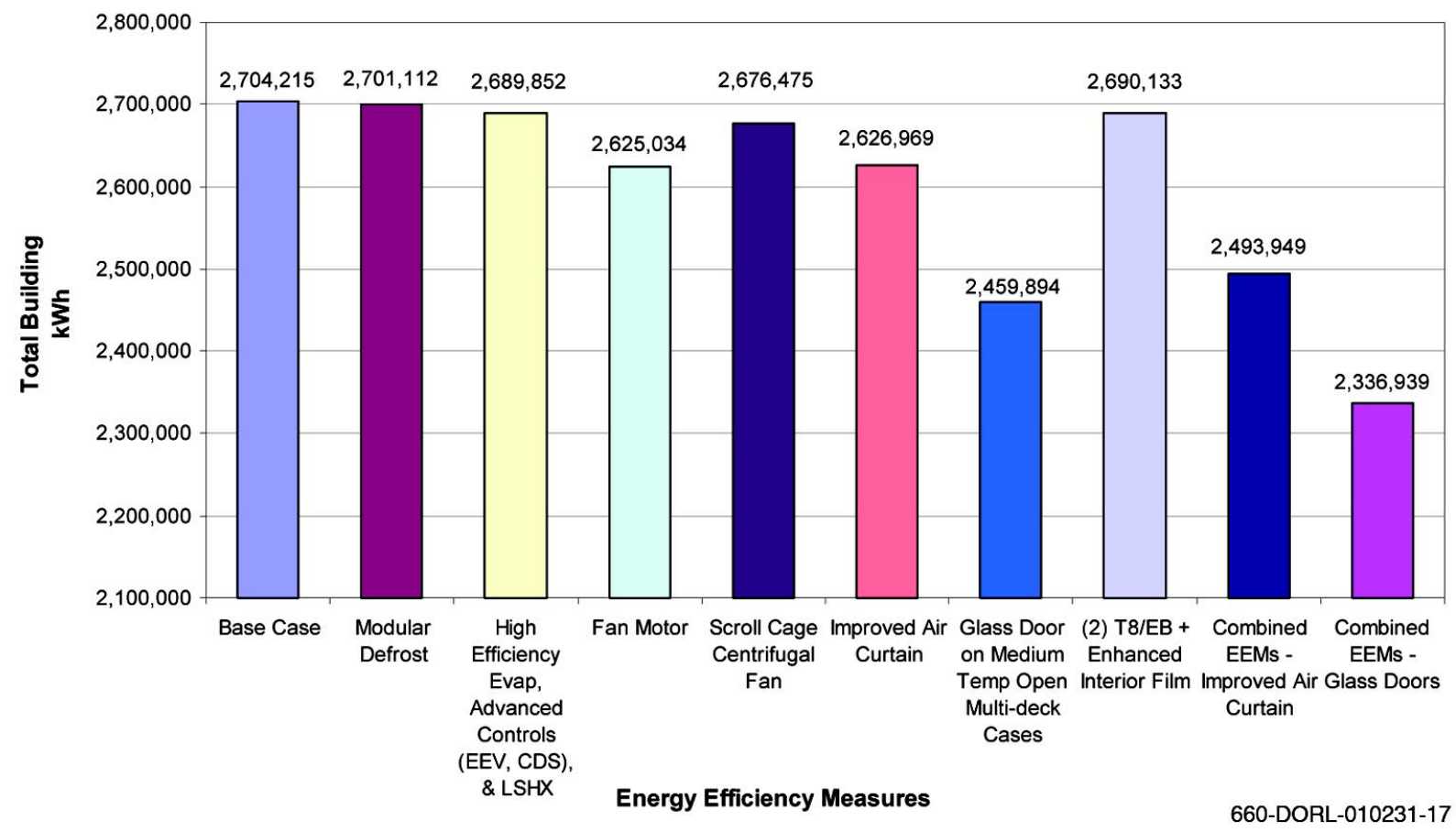

Figure 25. Impact of energy efficiency measures on total building energy

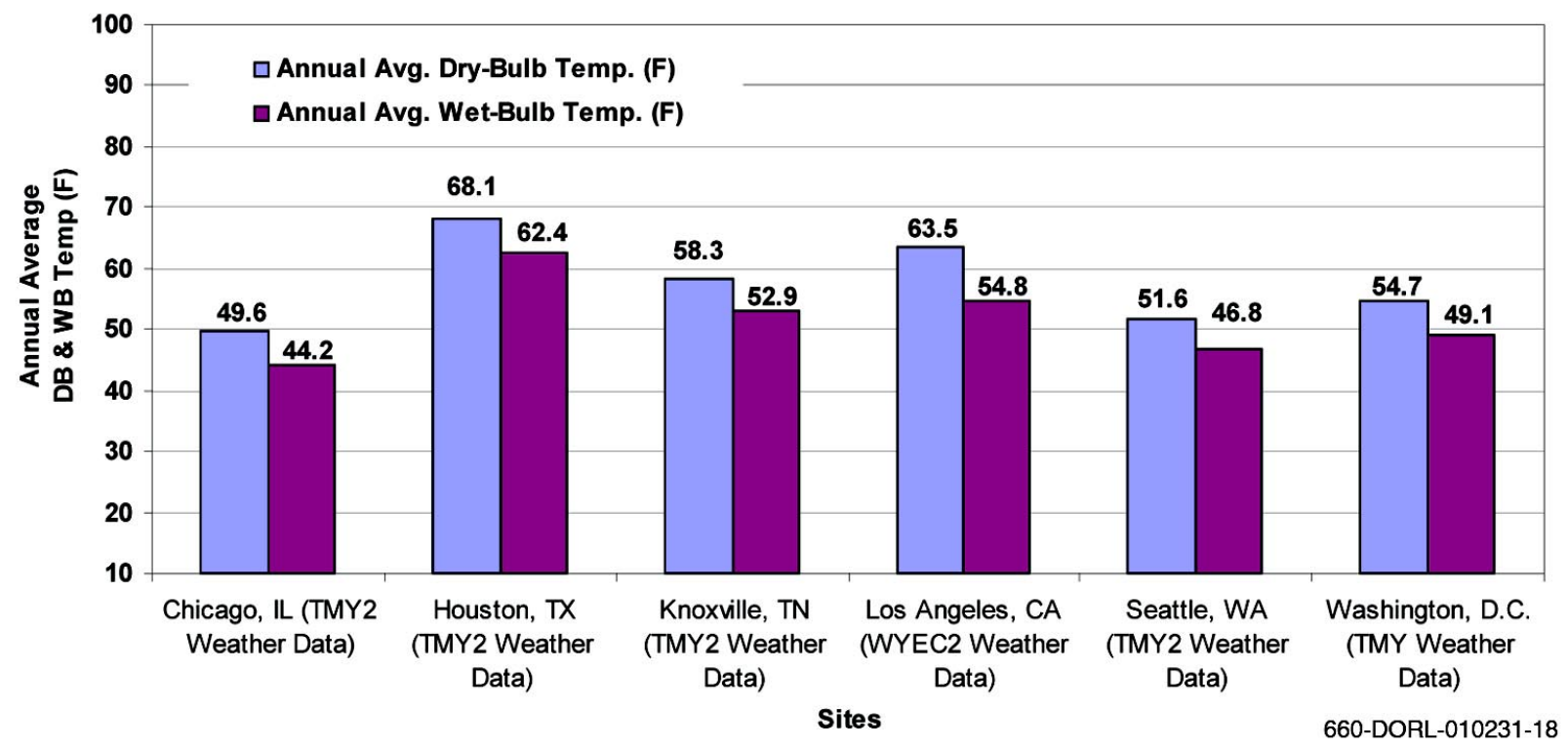

Figure 26. Annual average wet-bulb and dry-bulb temperatures for all six locations 
Comparing the base case annual cooling load and refrigeration energy for each location revealed that Houston had the highest and Chicago had the lowest cooling load (Figure 27) and refrigeration energy (Figure 28). In fact, the cooling load and refrigeration energy profiles for all six locations were similar to the weather profile.

Similarly, highest cooling load savings were observed for Houston and the lowest for Chicago (Figures 29 and 30). Figure 29 depicts the cooling load savings when base case and EEM 8 was compared. Figure 30, on the other hand, depicts the cooling load savings when base case and EEM 9 was compared. For all six locations, EEM 9 resulted in higher savings when compared to EEM 8.

\subsubsection{Summary of Simulation Results}

After the DOE-2.3 model was calibrated, all parametric simulations were performed using hourly weather data for California Thermal Zone 9, which represent Los Angeles. Modeling was performed for each individual EEM and for two combinations of EEMs. The first EEM combination (improved air curtain) included all individual EEMs. The second EEM combination (glass doors) included all of the technologies of the first combination with glass doors employed on open vertical cases instead of improved air curtains. The modeling results for the annual refrigeration energy for the baseline and when each of the EEMs are applied are shown in Figure 24. Comparing these annual energy consumptions showed the following annual refrigeration energy savings for each of the EEMs and EEM combinations:

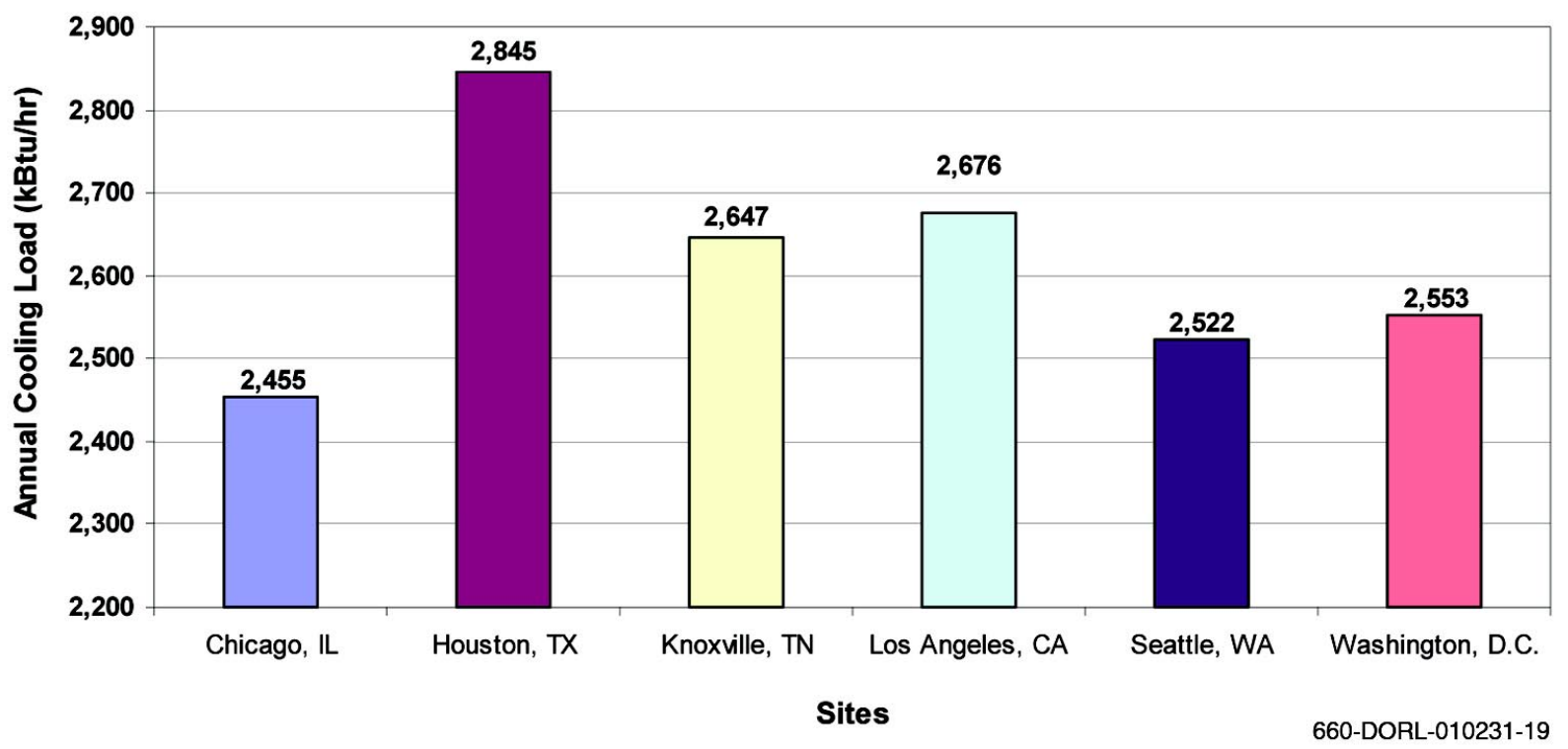

Figure 27. Comparison of annual cooling load for all six locations 


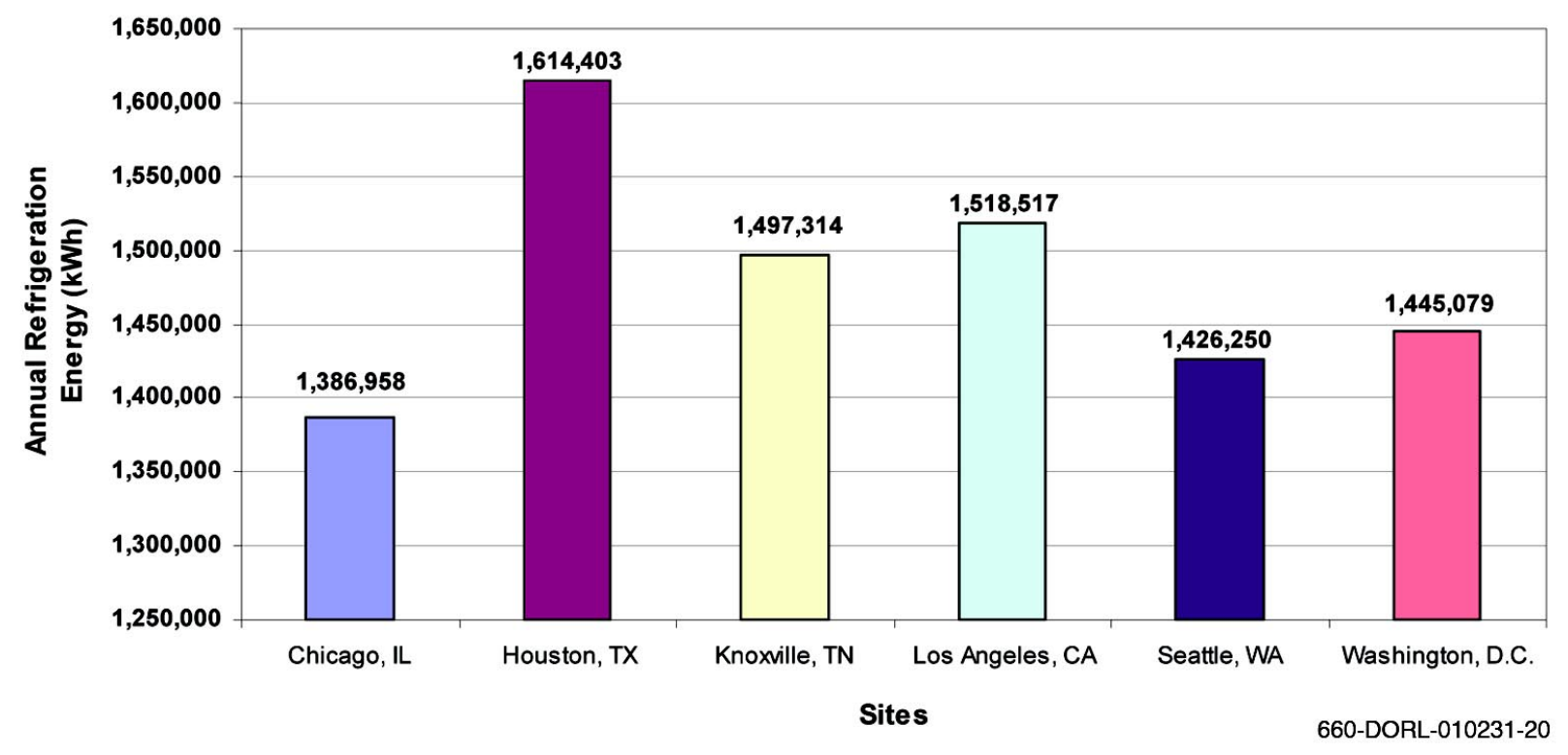

Figure 28. Comparison of annual refrigeration energy for all six locations

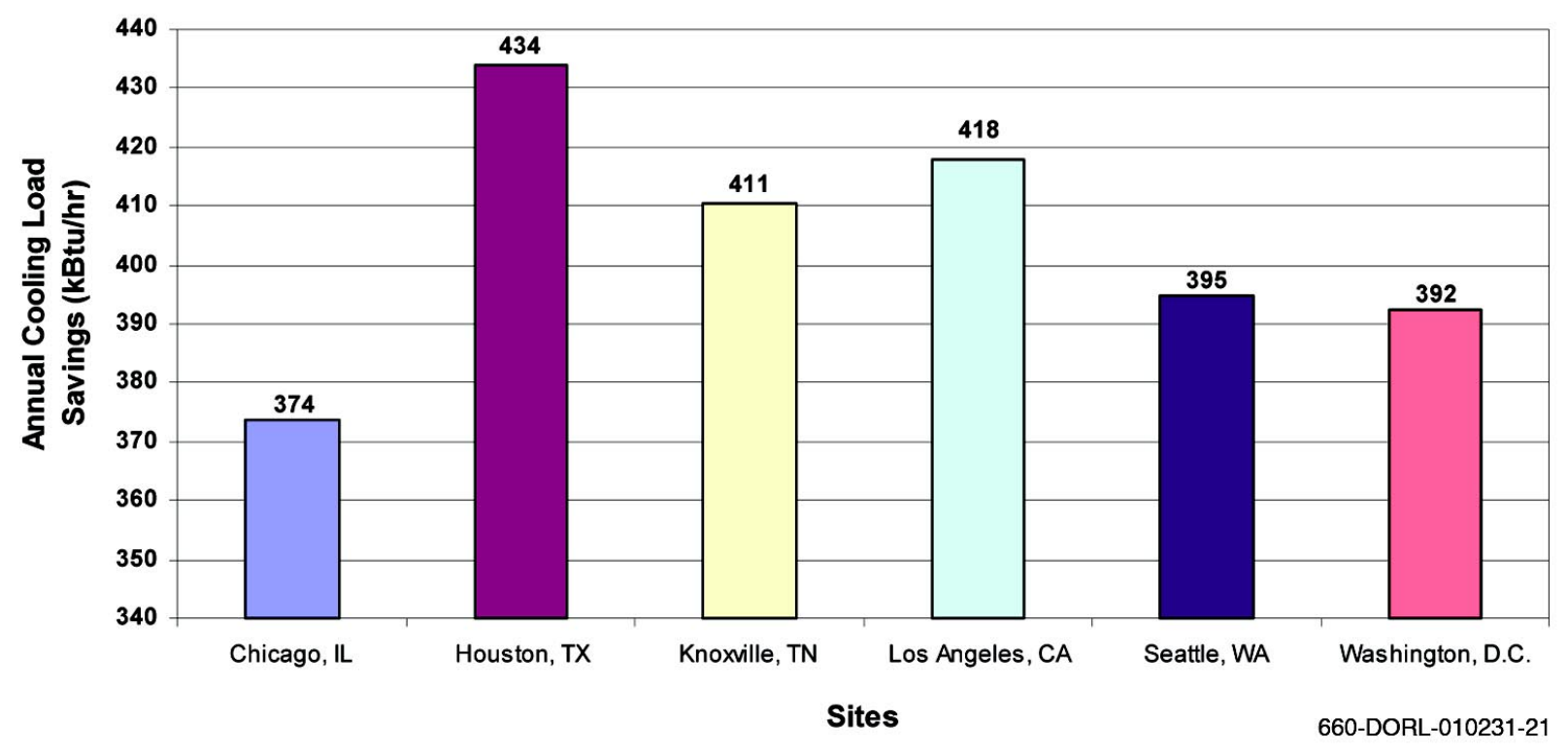

Figure 29. Comparison of annual cooling load saving per site (base case versus cumulative package with improved air curtain) 


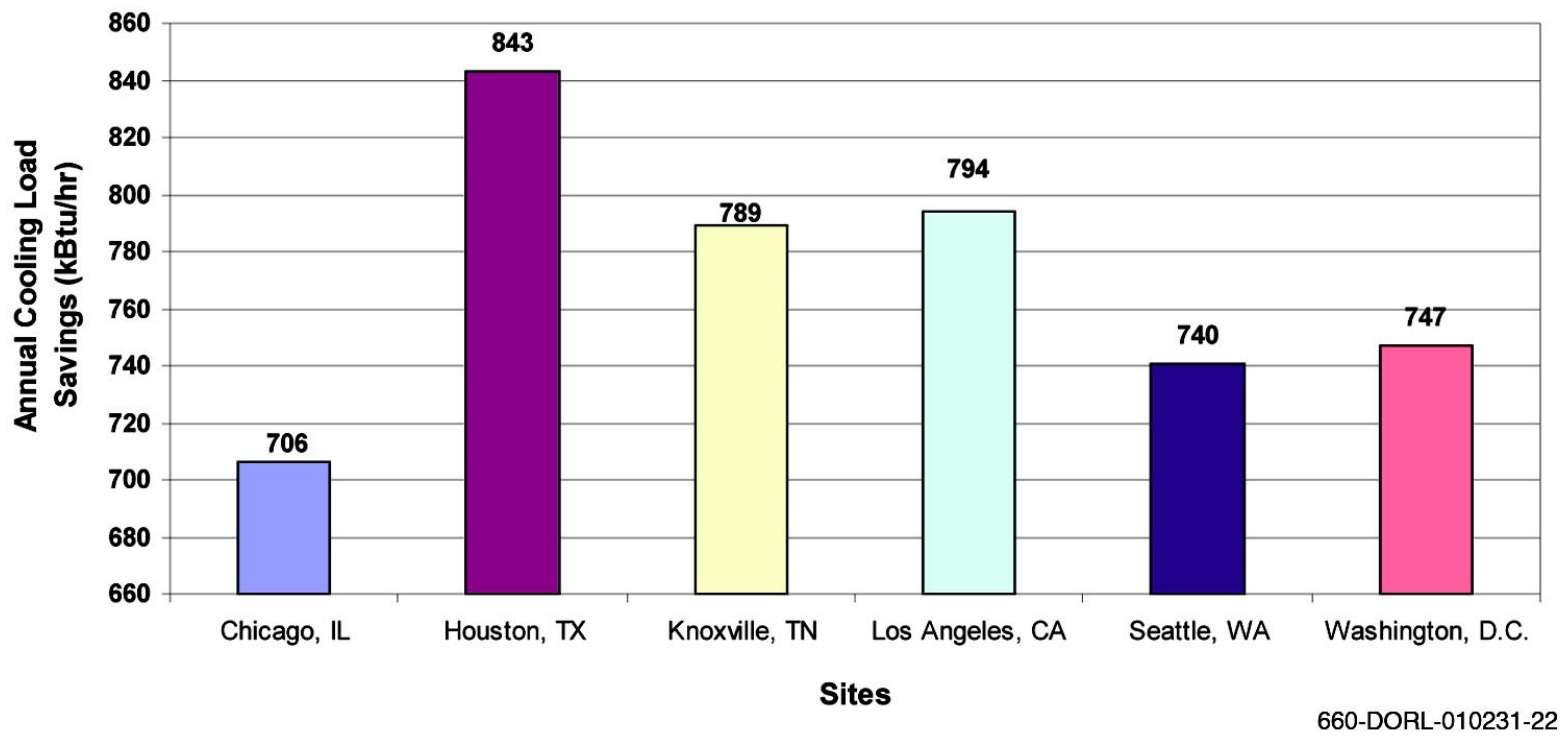

Figure 30. Comparison of annual cooling load saving per site (base case versus cumulative package with glass doors)

- Doors for open vertical medium temperature cases - 19.5 percent.

- Improved air curtain for open vertical cases - 5.5 percent.

- $\quad$ ECM fan motors - 5.3 percent.

- Scroll cage fans - 1.9 percent.

- Advanced evaporator with improved controls and LSHX - 1.0 percent.

- T-8 fluorescent lights with interior reflective film -0.9 percent.

- Modular defrost - 0.2 percent.

- Improved air curtain (first EEM combination) - 14.4 percent.

- Glass doors (second EEM combination) - 27.5 percent.

Modeling was also performed and compared for two combined EEMs (improved air curtain and glass doors) based on six different locations in nation. For all six locations, the second combination EEM (glass doors) generated higher cooling load savings when compared to the first combination EEM (improved air curtain). 


\section{INVESTIGATION OF AMBIENT AIR ENTRAINMENT IN THE DISPLAY CASE AIR CURTAIN}

\subsection{LDV Setup}

A Laser-Doppler Velocimeter (LDV) was used to measure the air velocity profile at two locations of the discharge air grill (DAG) of the display case air curtain. The LDV was located at the end of an extension arm attached to a computer controlled traversing system. The traversing system was arranged so that velocity measurements could be taken at selected points across the width of the DAG. The LDV setup is illustrated in Figure 31.

\subsection{DPIV Setup}

The DPIV technique was used to produce an instantaneous, two-dimensional, global velocity vector map of the flow field in a single plane of the flow. The technique can be used to obtain mean velocity information as well as turbulent statistics.

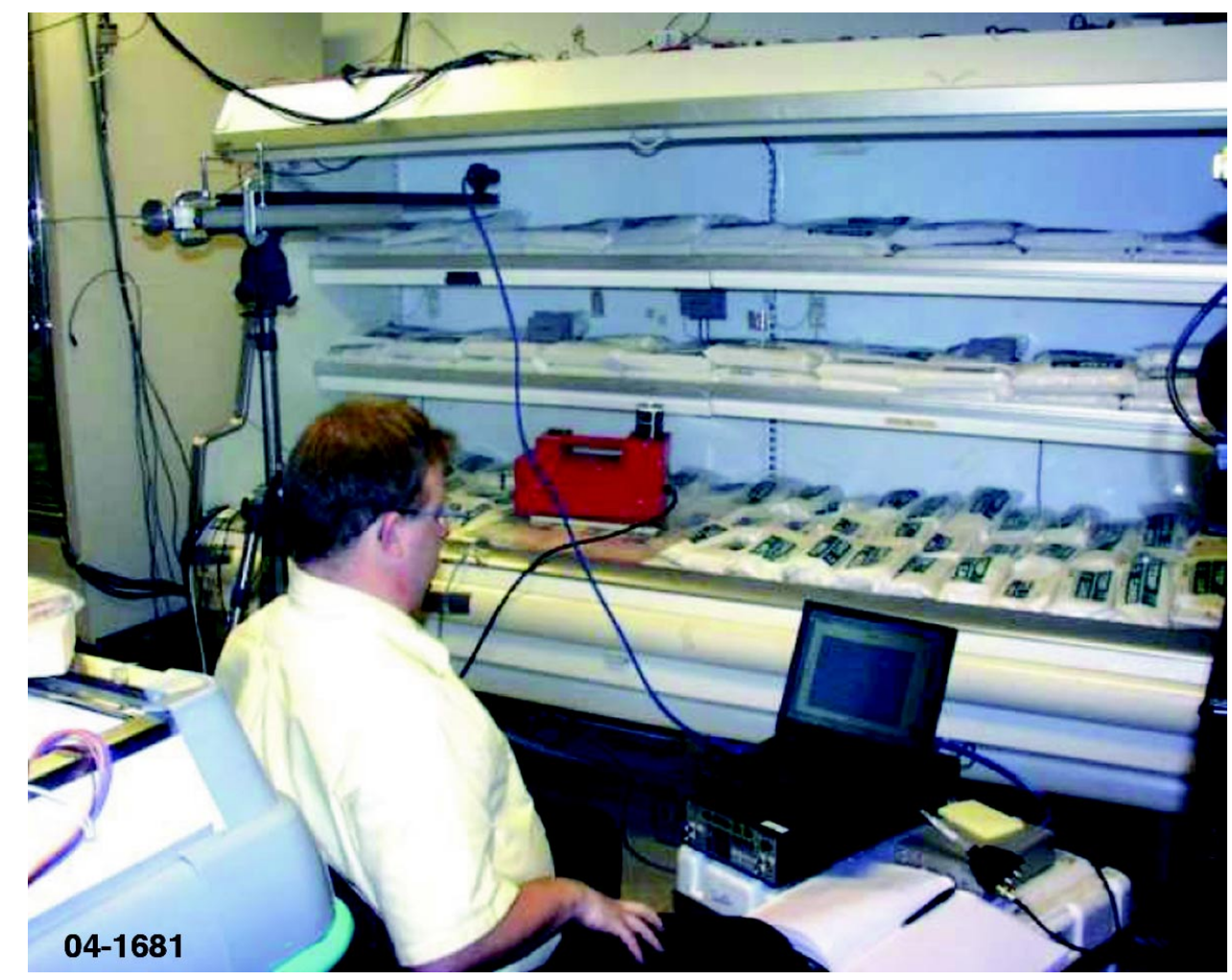

Figure 31. The LDV installation at the DAG of the display case 
A high-powered, dual-head, pulsed Nd:YAG laser is used to instantaneously illuminate a region of the flow field. The laser light is passed through suitable optics to form a light sheet. The pulse duration of these lasers is typically around $5 \mathrm{nsec}$. The flow is seeded with neutrally buoyant particles that are around 0.2 to $5 \mu \mathrm{m}$ in diameter for flows involving air. The laser light scattered from the seeded particles is imaged on a high-resolution digital video camera. Since the dual-head lasers have two individual laser heads, the flow can be illuminated twice with a time delay between the two pulses. The two separate pulses are recorded on different frames of the digital video camera and the motion of the particles during the elapsed time is determined by comparing the location of the particles on the two frames. The velocity vectors are determined from the particle motion and the time elapsed between the two frames. Figure 32 is a schematic of the DPIV experimental setup.

\subsection{DPIV and LDV Air Curtain Experiments}

\subsubsection{Observation of Air Curtain Behavior}

Figure 33 shows the DPIV vertical (or Y-component) velocity field taken in the middle of the display case. It can be seen that the jet originating from the DAG slows down and accelerates again in the vicinity of the middle shelf. The vertical velocity of the air curtain decreases again as it spreads over a wider region and after further mixing with the outside air accelerates as it moves into the RAG.

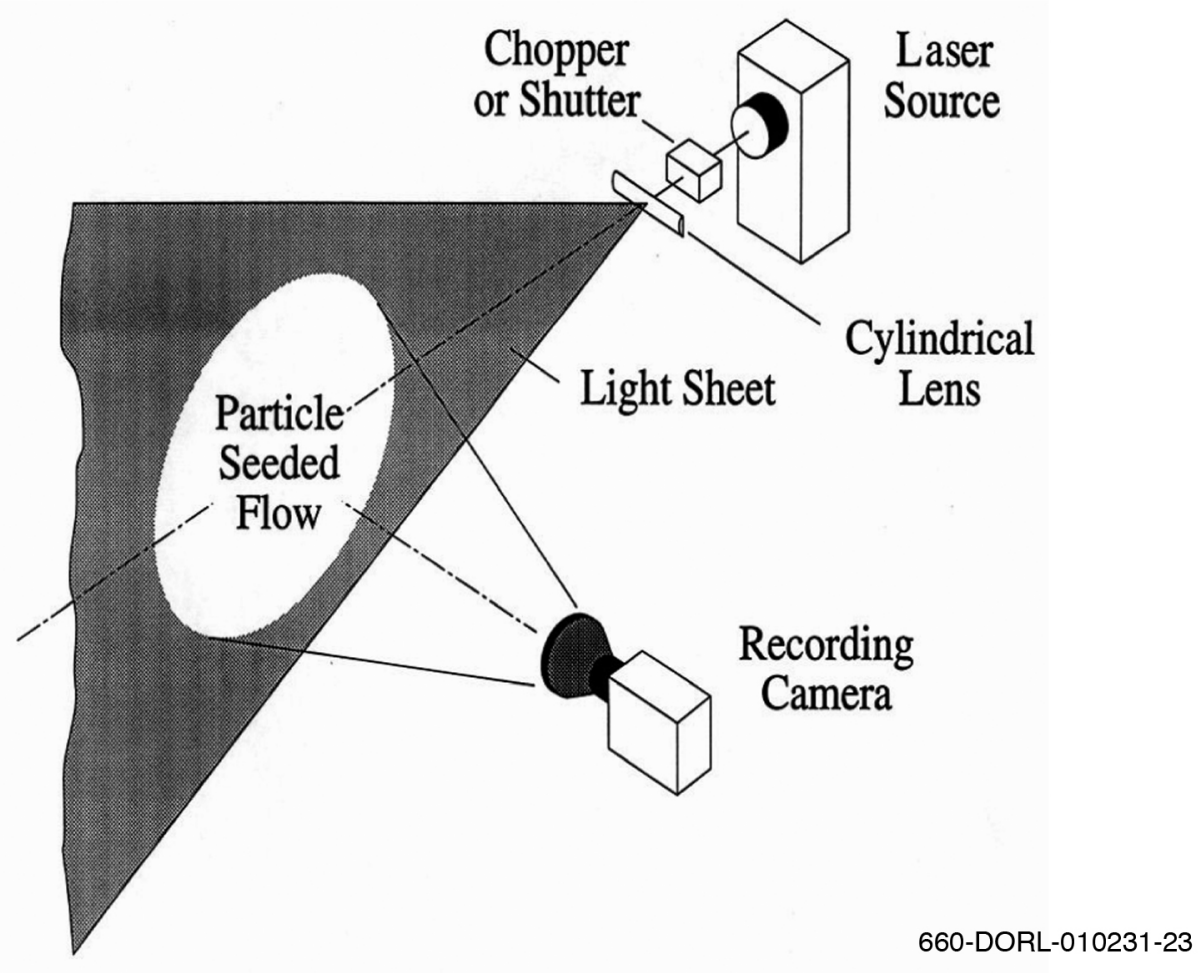

Figure 32. DPIV experimental setup 


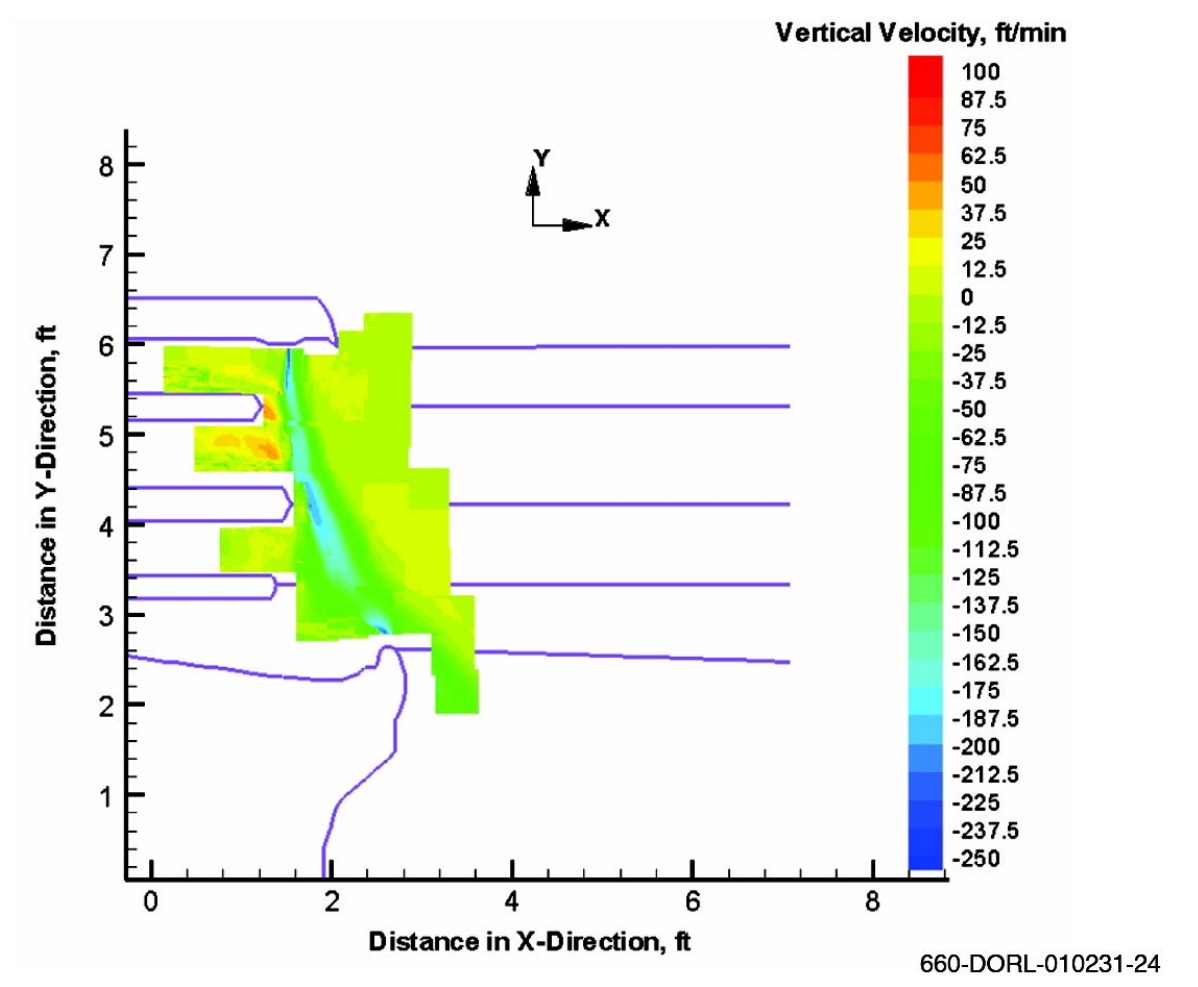

Figure 33. Vertical velocity field in the mid-plane of the display case by DPIV

The streamlines depicted in Figure 34 demonstrate a continuous and almost horizontal inflow of warm air into the air curtain, therefore providing a fairly continuous mixing of the warm and cold air.

\subsubsection{Measurement of DAG and RAG Air Flow Rates}

The LDV and DPIV techniques were both utilized in parallel to ensure the accuracy of the mean velocity field at the DAG and RAG. These velocity fields were used to determine the DAG and case (or total) airflow rates.

The LDV velocity measurements at the DAG were taken at two locations, which were 1.22 and $1.83 \mathrm{~m}$ ( $4 \mathrm{ft}$ and $6 \mathrm{ft}$ longitudinally) from one end of the case. The DPIV was used to measure the velocity profiles at the DAG and RAG at the middle of the case, or approximately $4 \mathrm{ft}$ from the end. The measured air velocity profiles of the DAG at these locations for both the LDV and DPIV are shown in Figure 35. The 0 in. DAG position in the figure corresponds to the inside of the display case. The velocity profiles shown are for the vertical component only. The horizontal component was also measured and found to be very small. Flow rates at the DAG and RAG can be estimated with reasonable accuracy using only the vertical velocity values. It should be noted that all velocity profiles shown are close enough so that the flow along the DAG (8-ft long case) in this particular display case can be assumed to be essentially two-dimensional. An excellent agreement between the two experimental methods can be observed. It can also be seen that both methods predict two peaks (or maximums) for the vertical velocity that corresponds to two tangential jets at the DAG. This two-peak profile results in increased 


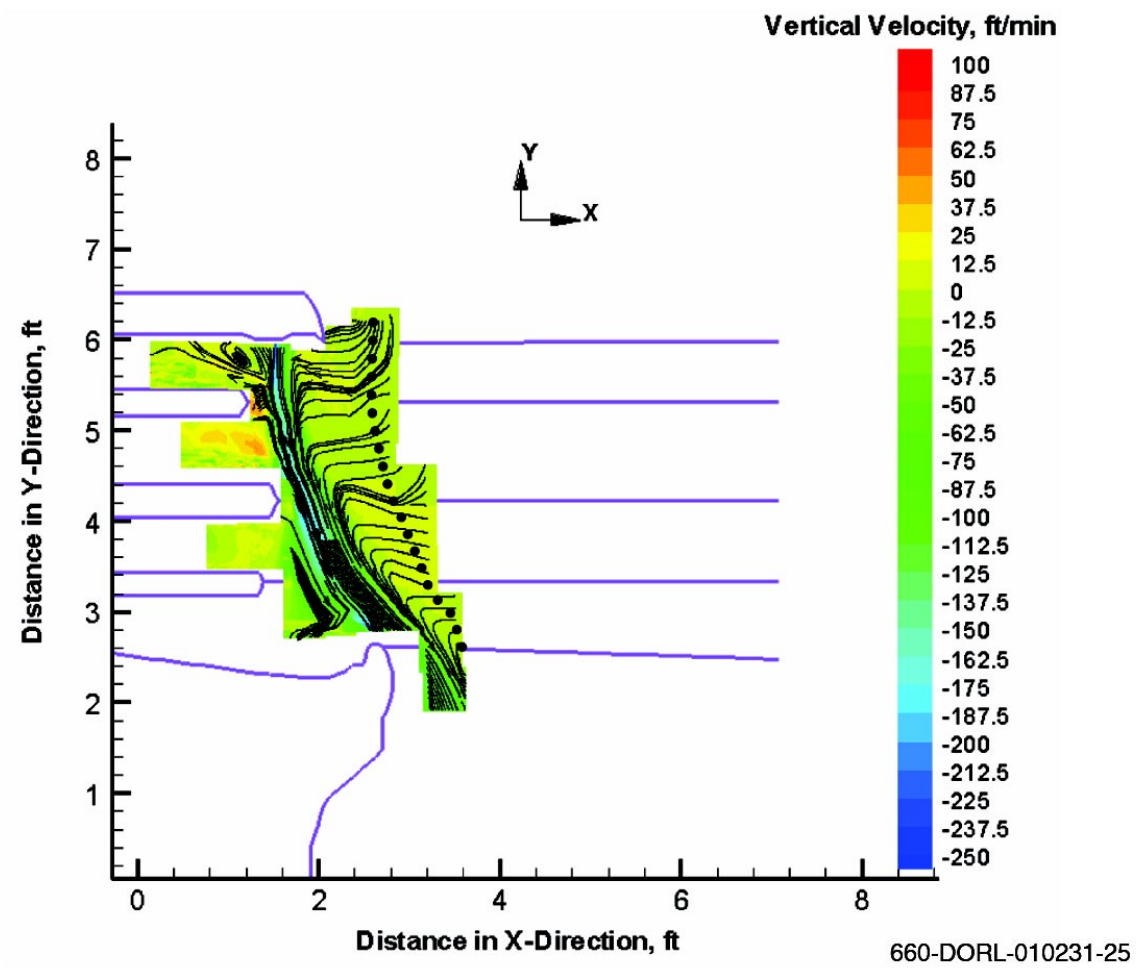

Figure 34. The display case mid-plane streamlines at steady state by DPIV (dotted line represents the surface used for the determination of total flow of entrained ambient air in Equation 5)

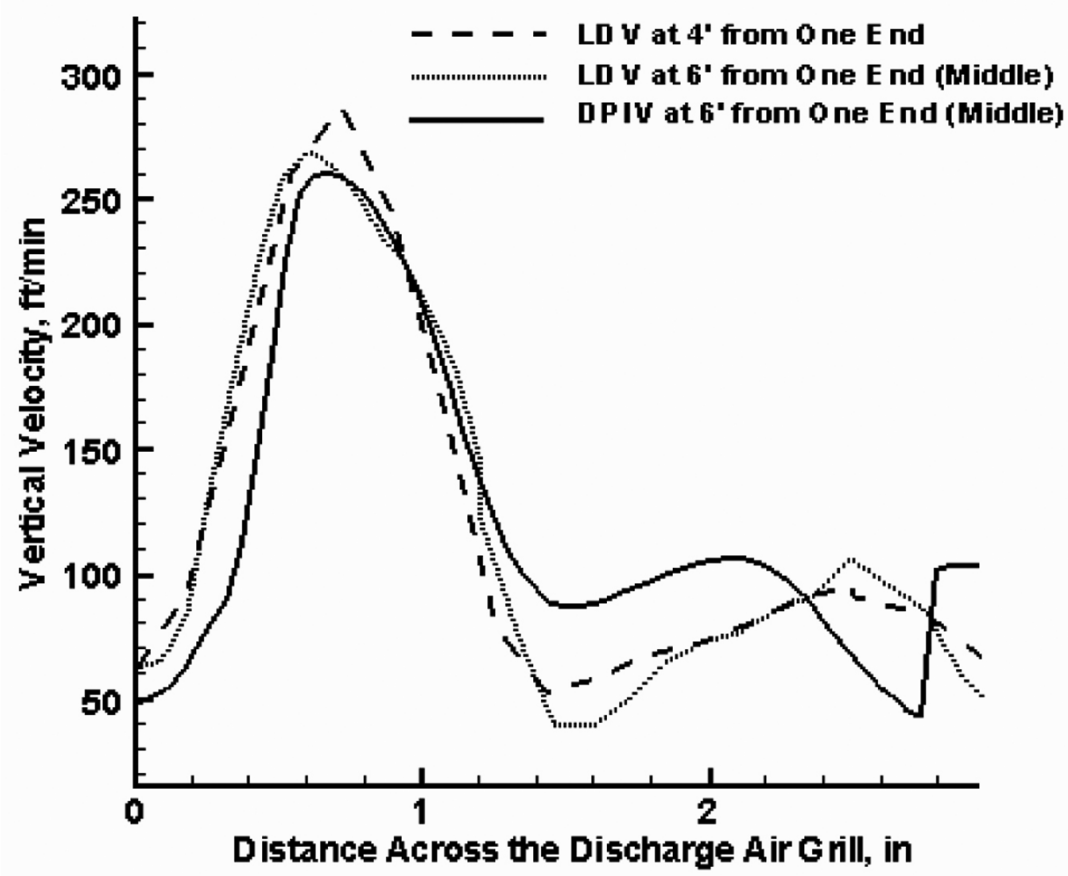

660-DORL-010231-26

Figure 35. Vertical velocity profile by LDV at two separate locations and by DPIV in the mid-plane of the display case. (The 0 in. DAG position in the figure corresponds to the inside of the display case) 
turbulent kinetic energy leading to increased turbulence intensity and ambient air entrainment rate, as will be discussed later.

The volumetric airflow rate supplied to the fixture through the DAG can be obtained from the following equation:

$$
\dot{\forall}=\int_{0}^{\mathrm{w}} v \mathrm{dA}
$$

where:

$\dot{\forall}=$ volumetric flow rate

$v \quad=$ the DAG velocity profile

$\mathrm{dA}=\ell \mathrm{dw}$ is the differential area with $\ell$ being the length of the display case

(8 $\mathrm{ft}$ in this case)

$w=$ width of the DAG

The velocity profile at the RAG can also be integrated to yield the flow rate that is handled by the fans (total display case flow rate). Therefore, the following equation can be applied:

$$
\dot{\mathrm{m}}_{\mathrm{RAG}}=\dot{\mathrm{m}}_{\mathrm{DAG}}+\dot{\mathrm{m}}_{\text {backpanel }}
$$

If the density of the air is assumed constant, the above equation can be converted into the volumetric flow rate, that is:

$$
\dot{\forall}_{\text {RAG }}=\dot{\forall}_{\text {DAG }}+\dot{\forall}_{\text {backpanel }}
$$

where back panel refers to the cold air delivered to the case through the perforated wall behind the shelving.

The difference between the RAG (total) and DAG mass flow rates will determine the amount of air coming through the back panel.

The average volumetric flow rate at the DAG was calculated to be about $7.93 \mathrm{~m}^{3} / \mathrm{min}$ $\left(280 \mathrm{ft}^{3} / \mathrm{min}, \mathrm{cfm}\right)$.

The vertical velocity component at the RAG was measured by the LDV and DPIV methods and the results are shown in Figure 36. A good agreement can be observed, therefore the total display case mass flow rate could be calculated by integrating the total velocity at the RAG shown in Figure 36 over the entire area of the RAG. This yielded a flow rate of about $22.08 \mathrm{~m}^{3} / \mathrm{min}(780 \mathrm{cfm})$. These values gave a flow rate of about $14.16 \mathrm{~m}^{3} / \mathrm{min}(500 \mathrm{cfm})$ through the back panel. 


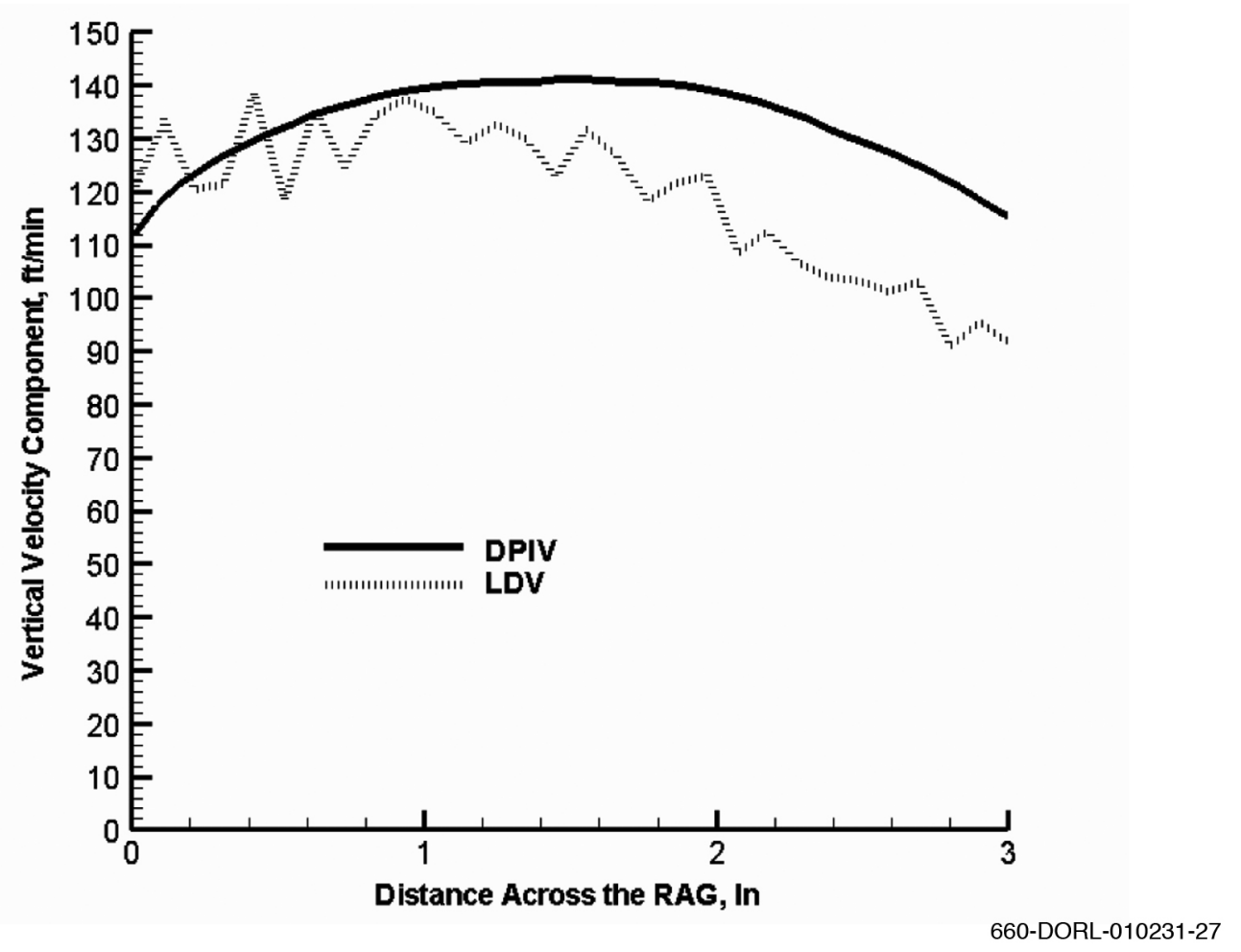

Figure 36. The vertical velocity component at the RAG by DPIV and LDV methods

The entrainment rate can be calculated by integrating the velocity over a surface where all streamlines are moving towards the display case. Figure 34 shows the streamlines that are moving towards the display case for DPIV results, i.e., the entrained air (The same streamlines as computed by the CFD model are shown later in Figure 41a). The following equation is used to find the entrainment rate:

$$
\dot{\mathrm{m}}_{\text {Entrained }}=\int_{0}^{\text {length }} \rho \ell \overrightarrow{\mathrm{V}}_{\text {total }} \bullet \mathrm{d} \overrightarrow{\mathrm{s}}
$$

If the density is considered to be constant, we get the entrainment volumetric flow rate:

$$
\dot{\forall}_{\text {Entrained }}=\int_{0}^{\text {length }} \ell \overrightarrow{\mathrm{V}}_{\text {total }} \bullet \mathrm{d} \overrightarrow{\mathrm{s}}
$$

In this equation, $\ell$ is the length of the display case ( $8 \mathrm{ft}$ in this case), $\overrightarrow{\mathrm{V}}$ is the total velocity vector and $\mathrm{d} \overrightarrow{\mathrm{s}}$ is the surface shown by the dotted line in Figure 34, where the streamlines are moving towards the display case. The dot product of these two will yield the entrainment rate. Upon performing this integration the total entrainment of ambient air into the air curtain was estimated to be $9.20 \mathrm{~m}^{3} / \mathrm{min}(325 \mathrm{cfm})$. 
The entrainment flow rate accounts for all ambient air that mixes with the air curtain. It must be noted that not all of this ambient air enters the display case through the RAG. Much of the mixed curtain and ambient air is spilled over the front of the display case. The ambient air that enters the RAG is considered the infiltration flow rate and cannot be determined directly through air velocity measurements. Instead, an energy balance must be taken that considers the air temperatures at the DAG and RAG, and of the ambient air. The energy analysis correlates the infiltration rate to the enthalpy or the average temperatures at these locations. The nomenclature for this analysis is shown in Figure 37.

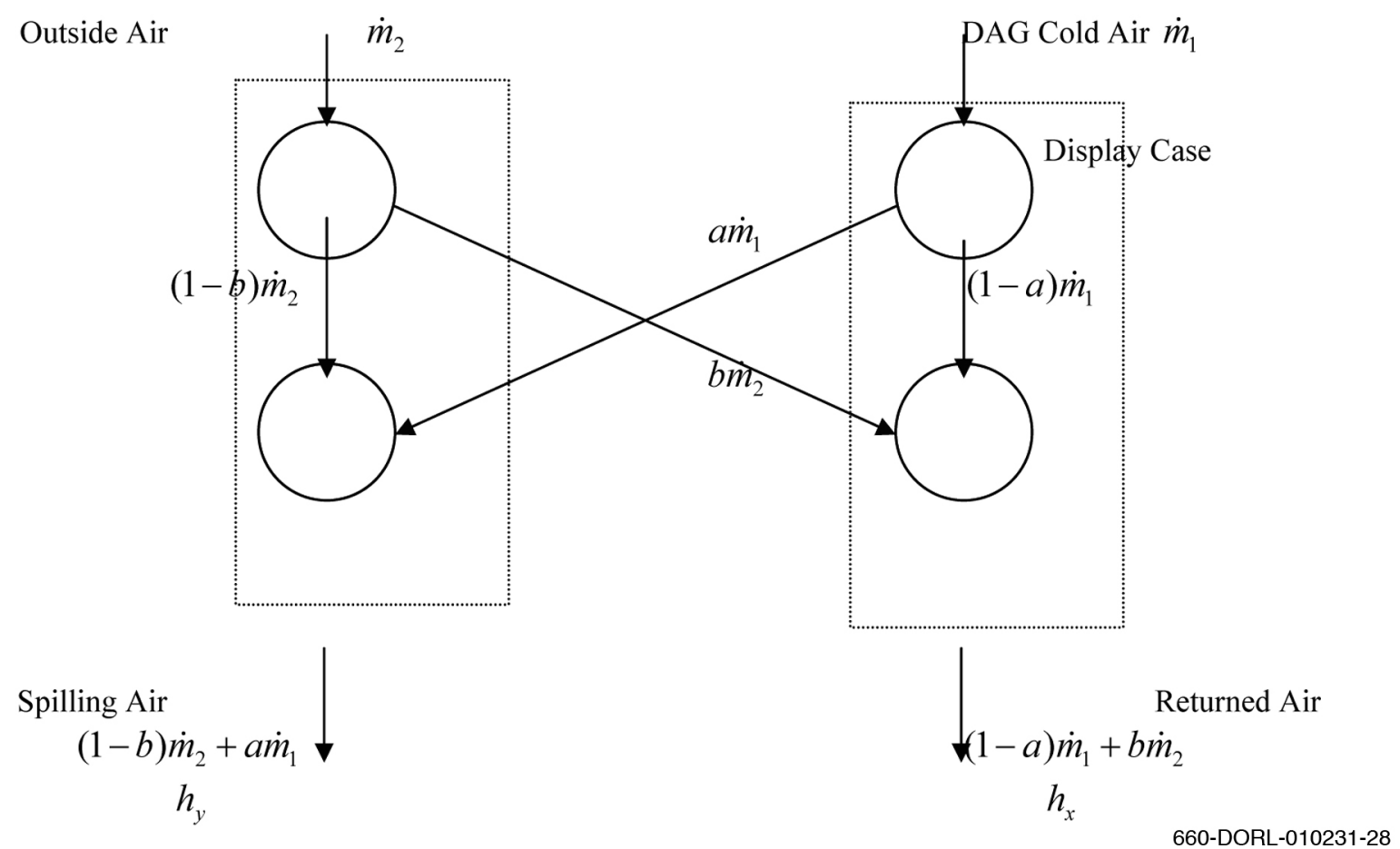

Figure 37. Nomenclature of the energy analysis used to determine the rate of air infiltration

where:

$\dot{\mathrm{m}}_{1}=$ Total mass flow rate into the case through DAG

$\dot{\mathrm{m}}_{2}=$ Mass flow rate of the outside air going towards the case (entrained air)

$\mathrm{a}=$ Portion of the air mass in the case that is mixed with the outside air (spillage)

$\mathrm{b}=$ Portion of the outside air that is mixed with the air mass in the display case (infiltration rate)

$\mathrm{h}=$ Enthalpy

Based on the fact that the total mass flow rate of the display case is constant, we have: 


$$
(1-\mathrm{a}) \dot{\mathrm{m}}_{1}+\mathrm{b}_{2}=\dot{\mathrm{m}}_{1}
$$

This will result in:

$$
\frac{\dot{\mathrm{m}}_{2}}{\dot{\mathrm{m}}_{1}}=\frac{\mathrm{a}}{\mathrm{b}}
$$

We can write the first law for the right control volume:

$$
\sum \dot{\mathrm{m}}_{1} \mathrm{~h}_{1}=\sum \dot{\mathrm{m}}_{\mathrm{e}} \mathrm{h}_{\mathrm{e}}
$$

where "i" and "e" refer to inlet and exit, respectively.

$$
\dot{\mathrm{m}}_{1} \mathrm{~h}_{1}+\dot{\mathrm{m}}_{2} \mathrm{~h}_{2}=\dot{\mathrm{a}}_{1} \mathrm{~h}_{1}+\left[(1-\mathrm{a}) \dot{\mathrm{m}}_{1}+\mathrm{b} \dot{\mathrm{m}}_{2}\right] \mathrm{h}_{\mathrm{x}}
$$

For the control volume on the left:

$$
a \dot{\mathrm{m}}_{1} \mathrm{~h}_{1}+\dot{\mathrm{m}}_{2} \mathrm{~h}_{2}=\mathrm{b} \dot{\mathrm{m}}_{2} \mathrm{~h}_{2}+\left[(1-\mathrm{b}) \dot{\mathrm{m}}_{2}+\mathrm{a} \dot{\mathrm{m}}_{1}\right] \mathrm{h}_{\mathrm{y}}
$$

With the help of the equation (1), we can solve for $a$ and $b$ :

$$
\begin{aligned}
& \mathrm{a}=\frac{\mathrm{h}_{\mathrm{x}}-\mathrm{h}_{1}}{\mathrm{~h}_{2}-\mathrm{h}_{1}} \\
& \mathrm{~b}=\frac{\mathrm{h}_{2}-\mathrm{h}_{\mathrm{y}}}{\mathrm{h}_{2}-\mathrm{h}_{1}}
\end{aligned}
$$

In terms of average temperature of the spilled air $\left(\mathrm{T}_{\mathrm{y}}\right)$ and returned air $\left(\mathrm{T}_{\mathrm{x}}\right)$, we have:

$$
\begin{aligned}
& \mathrm{a}=\frac{\overline{\mathrm{T}}_{\mathrm{x}}-\mathrm{T}_{1}}{\mathrm{~T}_{2}-\mathrm{T}_{1}} \\
& \mathrm{~b}=\frac{\mathrm{T}_{2}-\overline{\mathrm{T}}_{\mathrm{y}}}{\mathrm{T}_{2}-\mathrm{T}_{1}}
\end{aligned}
$$

The amount of the entrained air is " $b$ " and is a function of the temperatures of the outside air $\left(T_{2}\right)$ and DAG supply air $\left(T_{1}\right)$, and the average temperature of the over spilled air $\left(\overline{\mathrm{T}}_{\mathrm{y}}\right)$. Let us examine Equation (13). For prescribed outside air and DAG temperatures, the denominator is constant. If $\overline{\mathrm{T}}_{\mathrm{y}}$ is increased (that is the temperature of the over spilled air), "b" will decrease 
meaning less warm air is infiltrated into the case (most of it is spilled so that $\overline{\mathrm{T}}_{\mathrm{y}}$ gets closer to $\mathrm{T}_{2}$ ) therefore a lower RAG temperature should be expected. On the other hand, lower $\overline{\mathrm{T}}_{\mathrm{x}}$, i.e., RAG temperature from Equation (12) implies smaller "a" that is smaller amount of overspill of the cold DAG air. This is consistent with the physics of the problem.

From the air curtain evaluation testing, the DAT was maintained at $29^{\circ} \mathrm{F}$. The room temperature was $75^{\circ} \mathrm{F}$, and the average temperature of air spilling over the display case, obtained by integration, was calculated to be about $59.5^{\circ} \mathrm{F}$. Using these values in Equation (13), the amount of infiltrated warm air into the RAG can be calculated to be:

$$
\mathrm{b}=(75-59.5) /(75-29)=0.337 \text { or } 33.7 \%
$$

By calculating the average temperatures of over spilled and returned air, about 30 to 35 percent of the entrained air was infiltrated into the display case. So, about $3 \mathrm{~m}^{3} / \mathrm{min}$ $(106 \mathrm{cfm})$ of ambient air imposed a cooling load on the display case.

\subsubsection{Measurement of Air Curtain Turbulence Intensity}

Turbulence enhances the amount of "mixing" in a flow. For a typical jet, mixing occurs at the boundaries or interfaces. The turbulence intensity (i) is a measure of the mixing and is defined as the root mean square (RMS) of the velocity fluctuations divided by the mean flow velocity.

$$
\mathrm{i}=\frac{\sqrt{\frac{1}{3}\left(\overline{\mathrm{u}^{\prime 2}+\mathrm{v}^{\prime 2}+\mathrm{w}^{\prime 2}}\right)}}{\mathrm{V}_{\text {mean }}}
$$

where $\mathrm{V}_{\text {mean }}=$ is the local mean velocity $=\sqrt{\left(\mathrm{u}^{2}+\mathrm{v}^{2}+\mathrm{w}^{2}\right)}$ with $u, v$, and $w$ being the mean velocity component in each direction (x, y, $\mathrm{z}$ in Cartesian coordinates). Note that for our twodimensional analysis, $w$ and $w$ are both zero.

The turbulent kinetic energy is also a function of the velocity fluctuations and another indicator of the amount of mixing responsible for creation of eddies and "rolling" effects in the flow.

$$
\mathrm{k}=\frac{1}{2}\left(\overline{\mathrm{u}^{\prime 2}}+\overline{\mathrm{v}^{\prime 2}}+\overline{\mathrm{w}^{\prime 2}}\right)
$$

As mentioned previously, the velocity profile at the baseline case DAG contained two peaks (or maximums) that correspond to two tangential jets, This is a situation that should be avoided because of the production of turbulent kinetic energy at the boundary of the two jets. In this particular display case DAG, there are basically three interfaces or boundaries in a twodimensional plane, which consist of the two interfaces with inside and outside air, and one 
interface between the two maximums observed in Figure 35. Figure 38 verifies the fact that the turbulence intensity reached a maximum at the boundaries and interfaces. Therefore, it can be postulated that the "shape" (only one maximum), and the turbulence intensity present at the DAG will control the entrainment rate. A good agreement between the LDV and DPIV results for the prediction of turbulence intensity is also observed in Figure 38.

The mean velocity was measured directly by the LDV or DPIV techniques. This quantity can also be computed numerically. Our measurements also recorded all the velocity fluctuations, i.e., $u$, and $v$ ' about the corresponding mean velocity field. Then the equation for turbulence intensity can be used to generate Figure 38.

However, in the CFD code, by using the two-equation turbulence model, the turbulent kinetic energy is calculated and the quantity

$$
\left(\overline{u^{\prime 2}}+\overline{v^{\prime 2}}+\overline{w^{\prime 2}}\right)
$$

is evaluated from the above equation and then used in the equation for the turbulence intensity.

Based on the above results, the examination of the entrainment rate as a function of the turbulence intensity at the DAG becomes indispensable. This was accomplished by performing parametric simulation using CFD codes. LDV and DPIV measurements were taken to define the

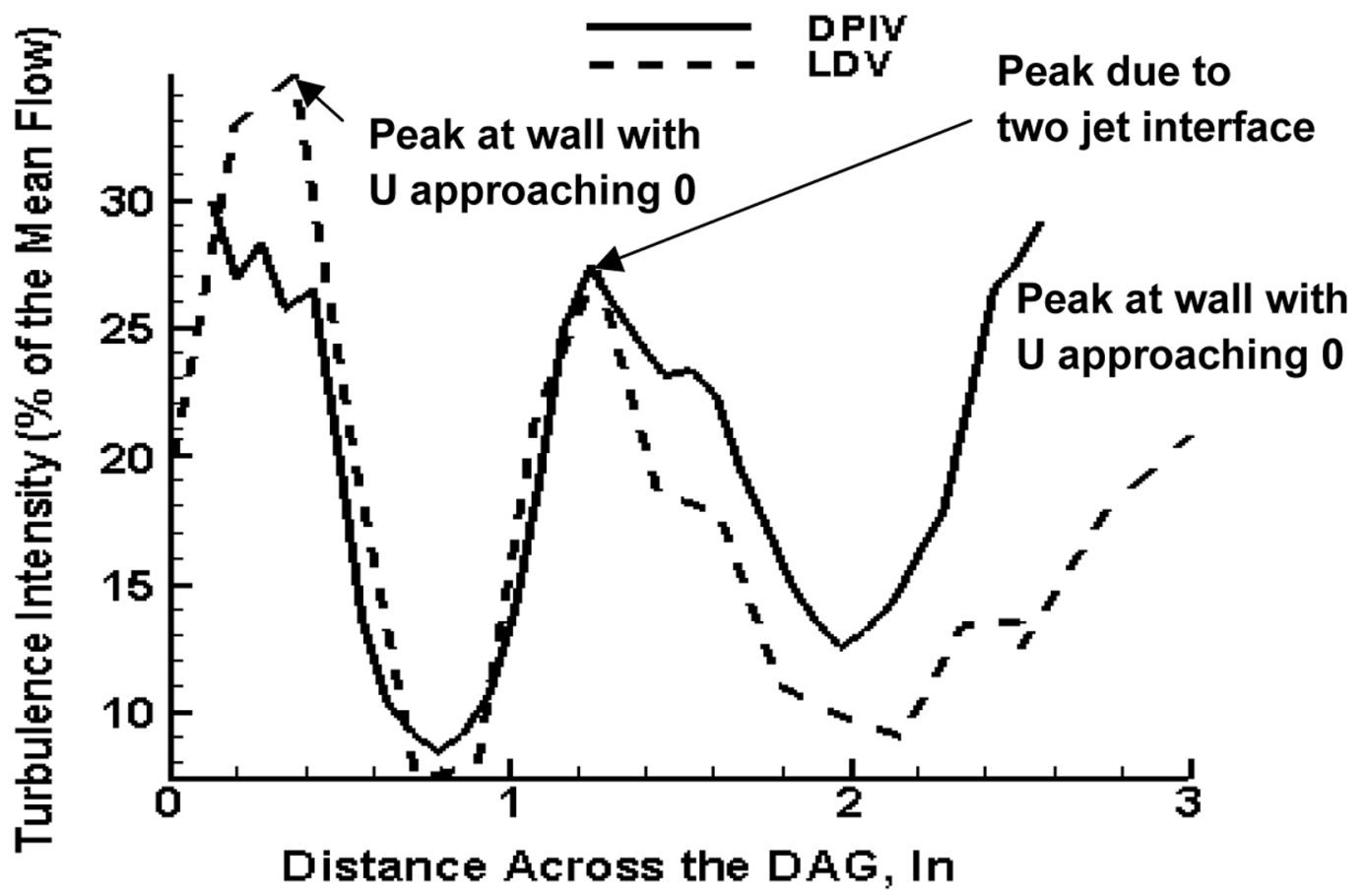

660-DORL-010231-29

Figure 38. Turbulence intensity at the DAG by LDV and DPIV methods 
inlet and exit boundary conditions (DAG and RAG) for the CFD codes. Figure 39 provides the point-wise vertical velocity profile at the RAG for the CFD simulation. The horizontal velocity profile that is not shown here is also an input to the CFD code. It should also be mentioned that the horizontal component of the velocity is very small and it does not affect the solution significantly.

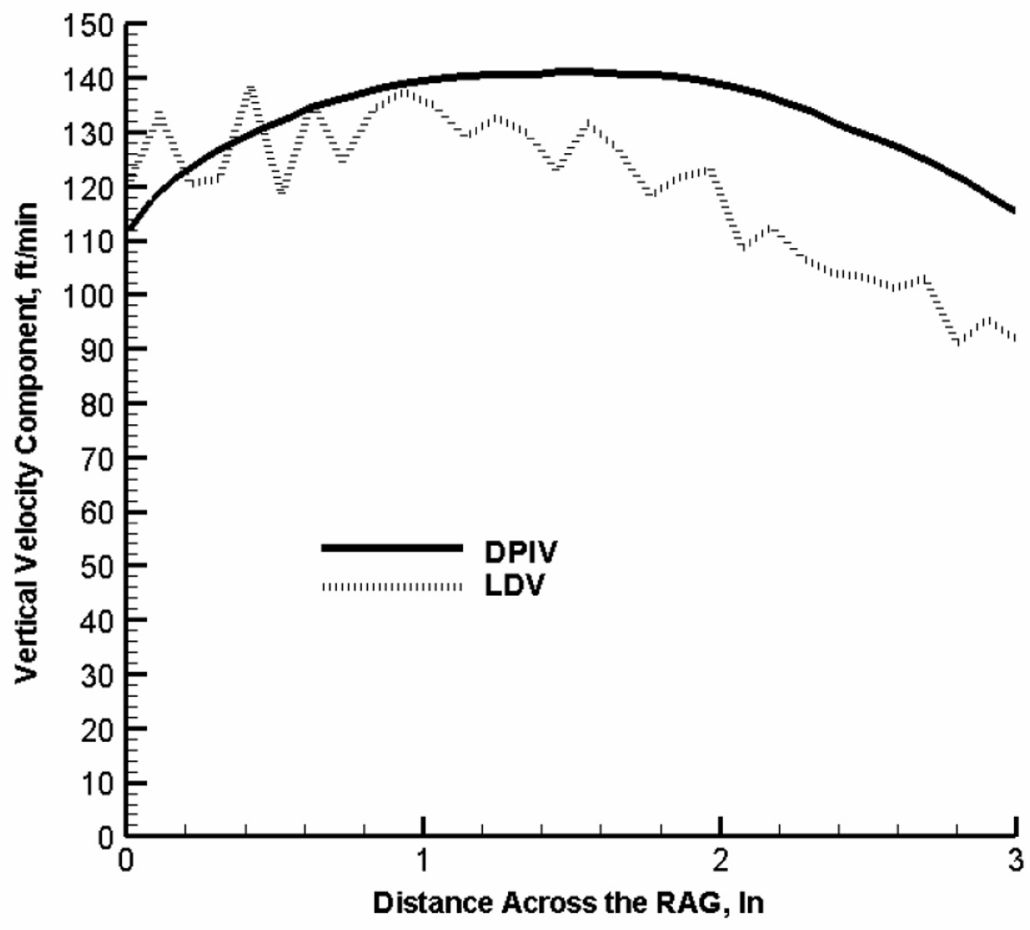

660-DORL-010231-30

\section{Figure 39. The vertical velocity component at the RAG by DPIV and LDV methods}

\subsection{CFD Simulation}

For the simulation of the flow field in the display case, two codes are used. The commercially available ${ }^{\odot}$ Fluent code $(\underline{11})$ is used to map the flow field in the entire domain, and the liquid thrust chamber performance $\left({ }^{\odot}\right.$ LTCP) code $(\underline{12})$ is mainly utilized for turbulence intensity studies due to its flexibility in specifying turbulent boundary condition. The effects of two important parameters, turbulence intensity and Reynolds number at the DAG inlet, on the entrainment and thereby infiltration rate were initially examined. For this evaluation, the Reynolds number was based on the DAG width.

\subsubsection{Modeling of Back Panel Airflow}

In the first computer run, the velocity profile at the DAG was taken from the DPIV results. Both, the ${ }^{\odot}$ Fluent and LTCP codes predicted the temperature contours very similarly to the infrared (IR) image of the test display case as shown in Figure 40. It can be seen that the shelves are fairly cold and the outside air does not penetrate into the shelves. The main reason for that is the 

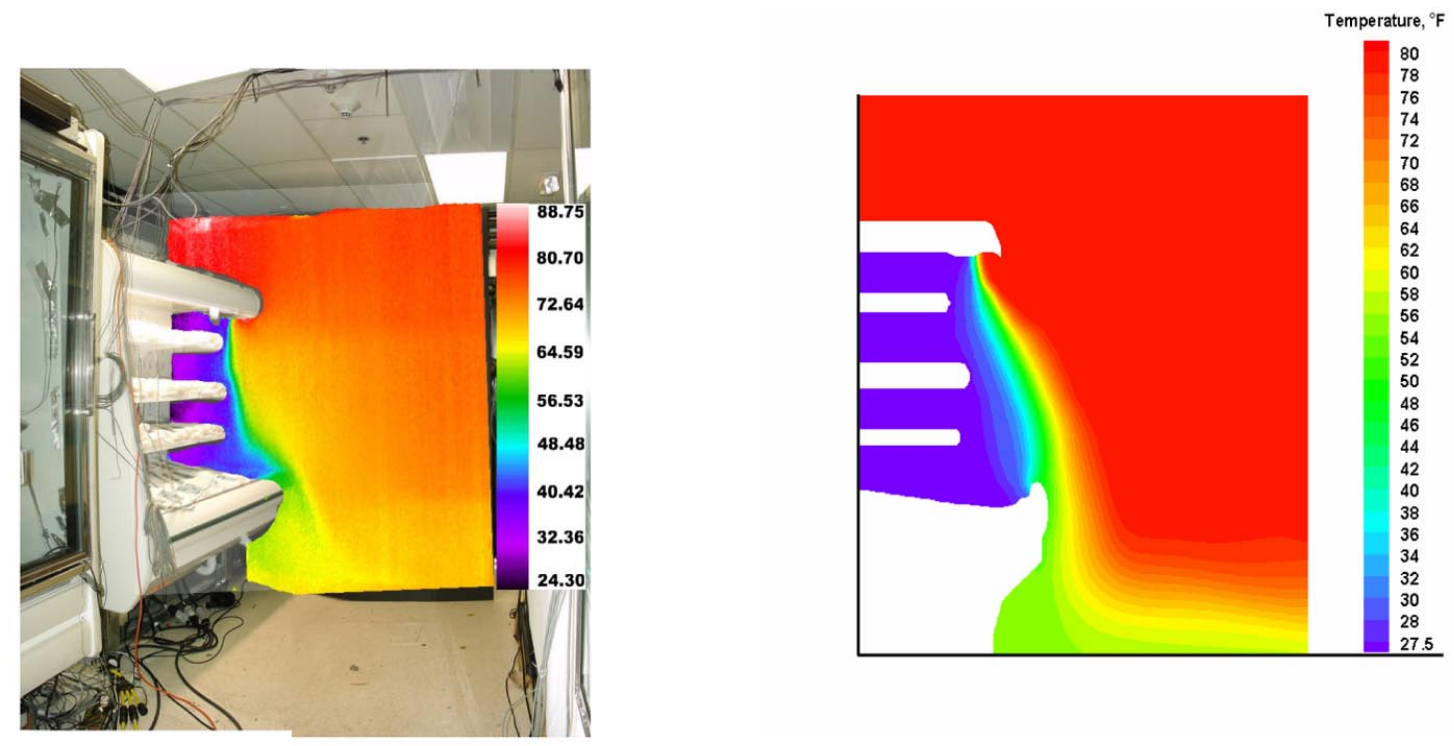

660-DORL-010231-31

\section{Figure 40. Comparison of CFD temperature field (left side) with infrared image (right side)}

flow through the back panel, and the fact that the air curtain is fairly effective in preventing the outside air from reaching the back of the shelves. However, in reality outside motions (by people reaching for food products) will cause instability of the air curtain and thereafter infiltration of the warm air into the case. Figure 41a shows the streamlines as predicted by the ${ }^{\circ}$ Fluent code. These are comparable to the DPIV measured results in Figure 34. There is a minor bulging seen in the lower part of the profile in Figure 41a. Since the exact mass flow rate through each compartment of the back panel was not known, the $14.16 \mathrm{~m}^{3} / \mathrm{min}(500 \mathrm{cfm})$ back panel flow was arbitrarily distributed amongst the shelves. It is possible that this assumption resulted in excessive airflow through the third shelf from the top. However, the basic behavior of the flow indicated an entrainment rate estimate from the CFD simulations of $9.06 \mathrm{~m}^{3} / \mathrm{min}$ $(320 \mathrm{cfm})$ that was very close to the measured DPIV results of $325 \mathrm{cfm}$.

The existence of the back panel flow helps the temperature to immediately decrease and stabilize after any intrusion. To demonstrate the effect of the back panel flow, we modeled no airflow through the back panel while maintaining the same volumetric flow rate of cold air through the DAG. The temperature distribution with no back panel flow is shown in Figure $41 \mathrm{~b}$ side by side with the original case (Figure 41a). It can be seen that a higher temperature is encountered in the lower shelves in the absence of the back panel flow. Furthermore, any intrusion may cause the warm air to penetrate into the lower shelves and become stagnant. If there is no flow to "push" or "drive" this warm air out, it may take a long time to lower the temperature in these shelves. 


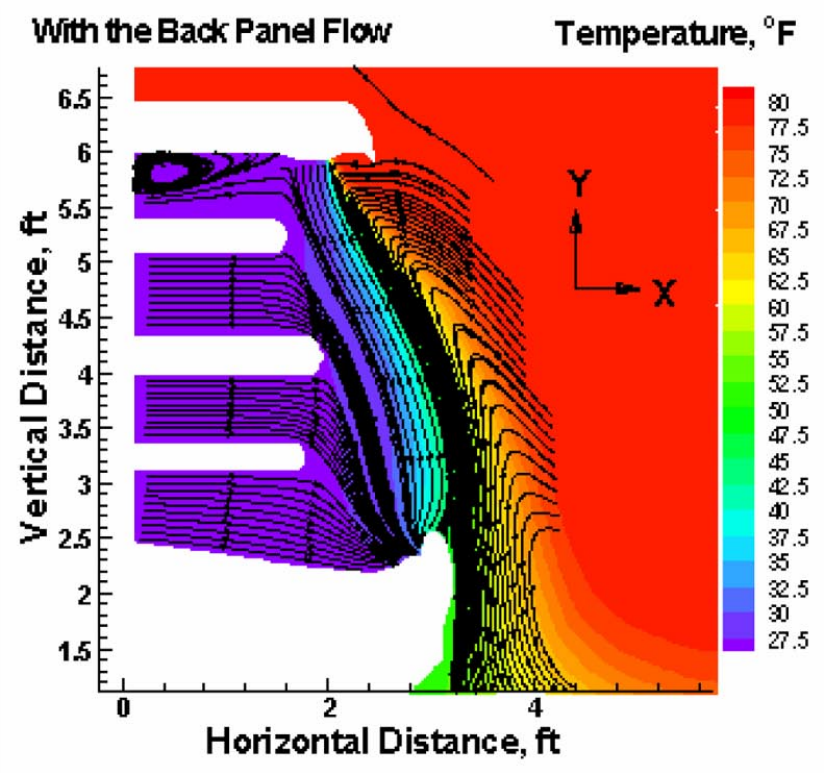

(a)

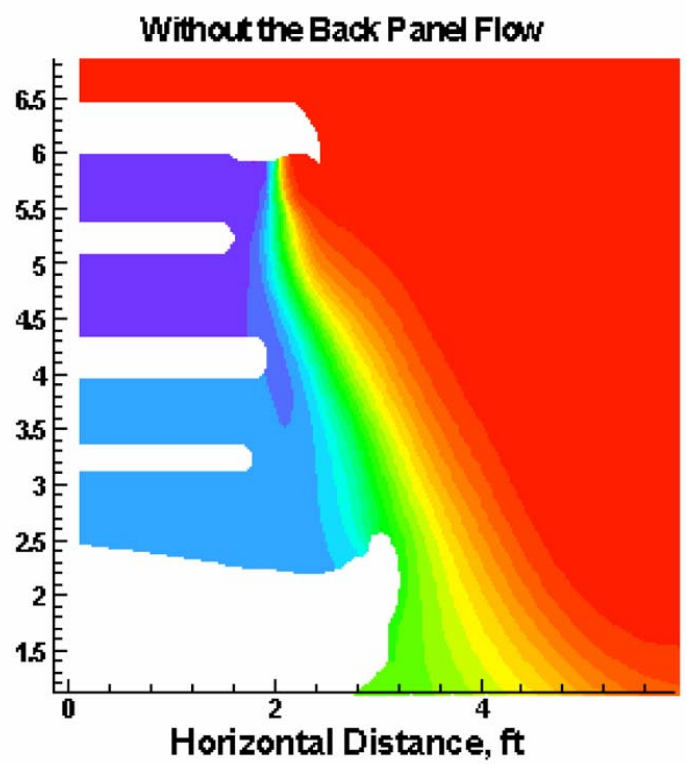

(b)

660-DORL-010231-32

\section{Figure 41. (a) Streamlines for the display case predicted by the ${ }^{\odot}$ Fluent code and temperature comparison with back panel flow present: (b) Temperature comparison with no back panel flow}

Therefore, it can be concluded that the back panel flow has a crucial effect on "stabilizing" the temperature in the shelves to an acceptable value after any intrusion or instability. However, a there is still a very important question: "What is the "minimum" required back panel flow necessary to maintain acceptable product temperature on the shelves?" As a test, we simulated the case with reduced back panel flow of $7.08 \mathrm{~m}^{3} / \mathrm{min}(250 \mathrm{cfm})$ and RAG (total) flow rate of $15.01 \mathrm{~m}^{3} / \mathrm{min}(530 \mathrm{cfm})$. For this change, the entrainment rate reduces to about $6.23 \mathrm{~m}^{3} / \mathrm{min}$ $(220 \mathrm{cfm})$ or about 42 percent of the total flow rate. However, the total flow rate is less than before with the same results, i.e., a small change in temperature at some locations in the shelves. The only factor that prevents reduction of the back panel flow in a real case is the amount of food products that are stored on the shelves and the mass of cold air that needs to be brought in for fast temperature stabilization after any intrusion.

\subsubsection{Modeling of Air Curtain Air Entrainment}

A series of simulations were performed to study the impact of the turbulence intensity and Reynolds number at the DAG on the entrainment rate. For all these simulations the back panel flow was maintained constant at $14.16 \mathrm{~m}^{3} / \mathrm{min}(500 \mathrm{cfm})$, and only the airflow at the DAG was changed. The results of this simulation are depicted in Figure 42. For higher Reynolds numbers at the DAG $(\geq 4200)$ the slope of the entrainment rate increased with turbulence intensity at the DAG. For the lowest Reynolds number, it seems that a slight turbulence actually reduces the entrainment rate. However, this Reynolds corresponds to a very low flow rate at the DAG, i.e., 


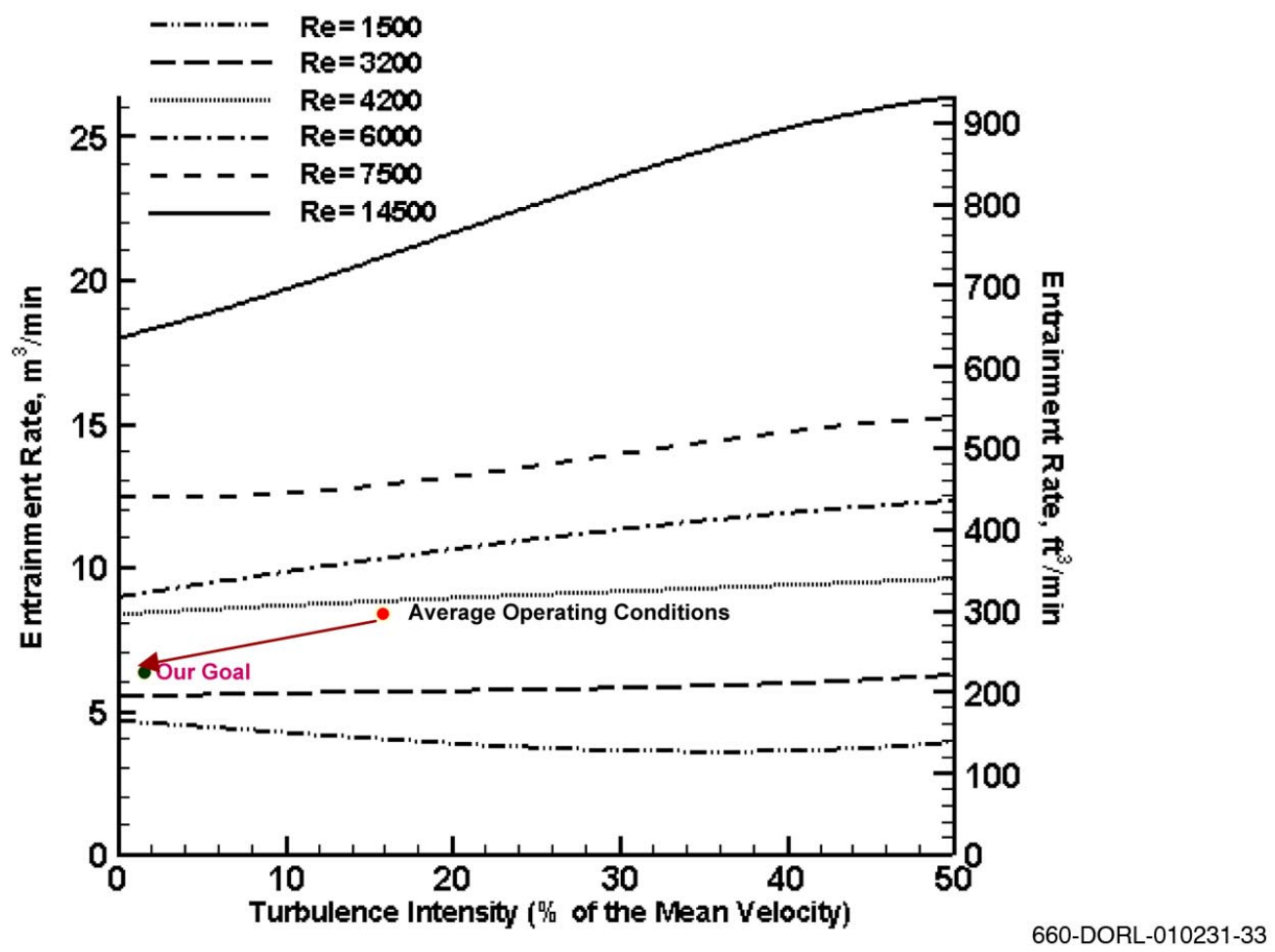

Figure 42. Entrainment rate for the display case as a function of turbulence intensity and Reynolds number at the $D A G$

$2.83 \mathrm{~m}^{3} / \mathrm{min}(100 \mathrm{cfm})$. For low Reynolds number, the stability of the air curtain becomes an issue. It can be seen that, for a Reynolds number of 3200, the effect of turbulence intensity at the DAG is minimized. It is recommended to run the DAG at a Reynolds number of about 3200 to 3400 to minimize ambient air entrainment without violating the stability of the air curtain structure.

From the above results, it can be concluded that both the turbulence intensity and Reynolds number at the DAG are major factors in determining the entrainment rate. This intensity is a function of the DAG geometry and the exit velocity profile at this location.

We have performed an extensive study to determine the entrainment rate as a function of these parameters while maintaining the same flow rate for all cases. Initially, we imposed several velocity profiles at the DAG with different turbulence intensities. Figure 43 shows all the profiles that were considered in our parametric studies. The parabolic profile basically represents a laminar flow in a perfectly symmetrical situation while the asymmetrical profiles can realistically exist due to the presence of shelves. However, the parabolic profile resembles a laminar flow profile, which is the desired flow condition at the DAG.

For the nominal operating conditions of the display case $(\mathrm{Re}=4300)$, the entrainment rates were calculated for all the DAG vertical velocity profiles shown in Figure 43. By examining the results of Figure 44, it is evident that the skewed parabolic profile at the DAG, with the peak shifted towards the inside of the display case (back panel), generated the second lowest 

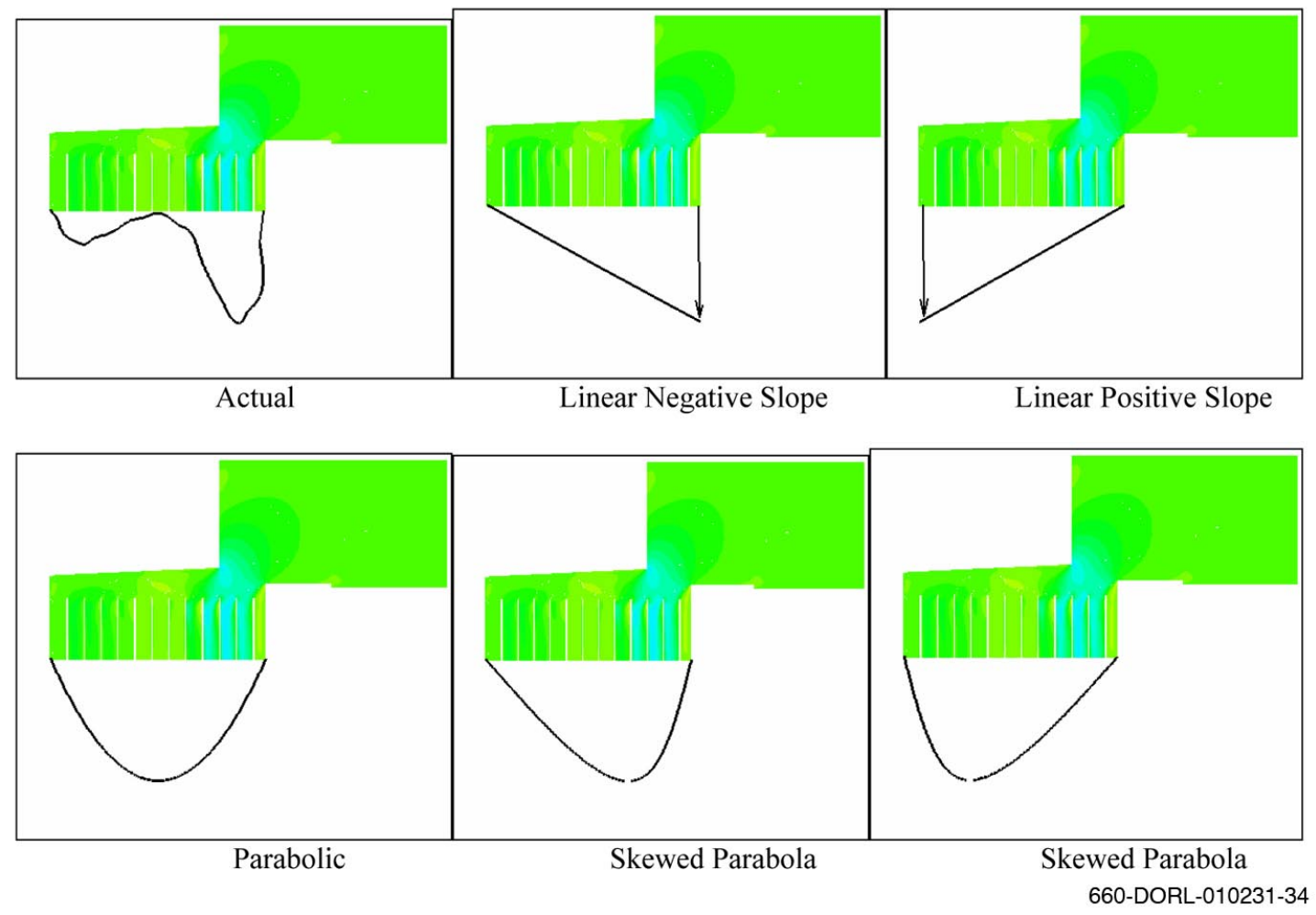

Figure 43. Schematic of velocity profiles under consideration for parametric studies

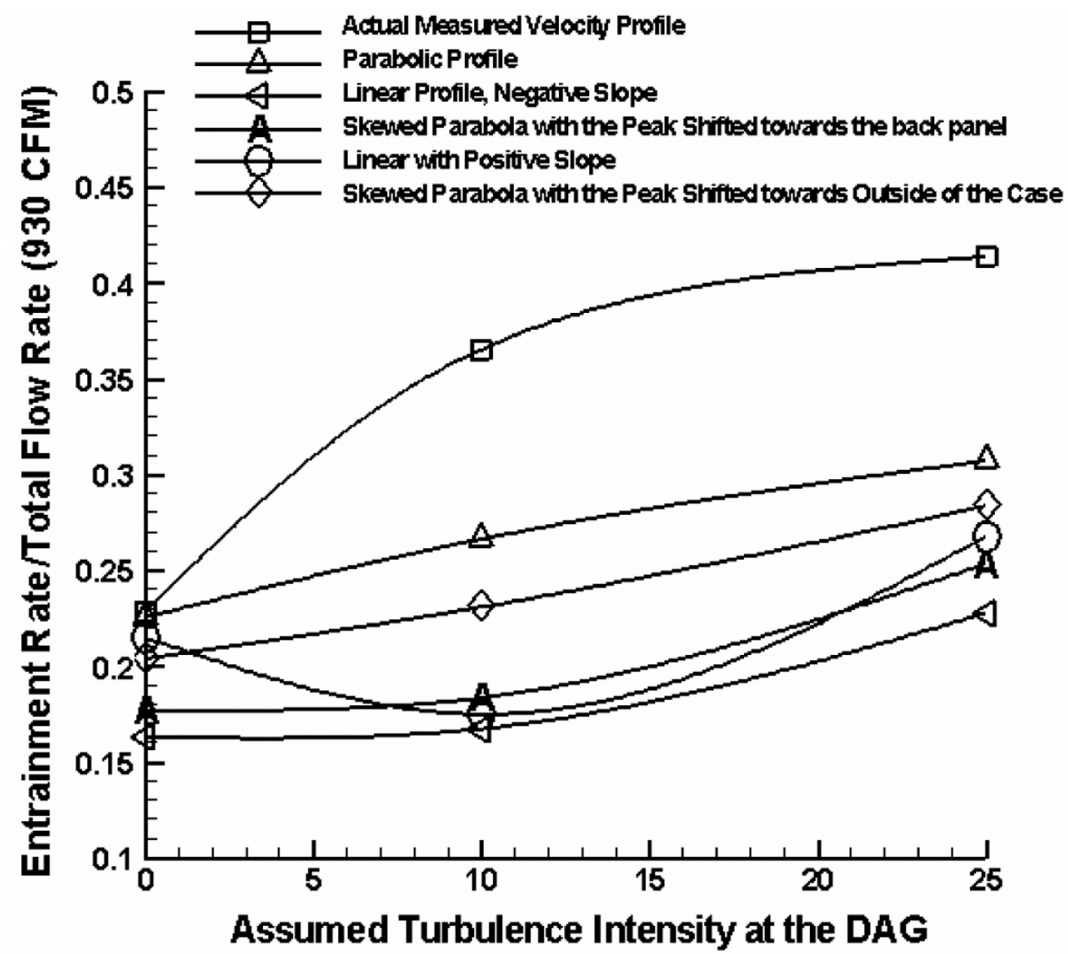

660-DORL-010231-35

Figure 44. Entrainment rate as a function of imposed turbulence intensity and different velocity profiles at the $D A G\left(\operatorname{Re}_{D A G}=4,300\right)$ 
entrainment rate (next to the linear profile with negative slope). Since achieving a linear velocity profile in a real DAG would be almost impossible, our goal was to determine the DAG configuration needed to produce the desired skewed parabolic profile.

The original DAG geometry consisted of an obstruction that created a narrow area for the flow to go through. These sharp edges will trigger the creation of turbulence and nonhomogeneous flow patterns. This fact can be observed in Figure 45 (actual geometry) where turbulent kinetic energy created at these sharp edges was about $0.85 \mathrm{~J} / \mathrm{kg}$ of air. This high value was maintained as flow progressed through the honeycomb. By examining the original geometry we concluded that the vertical obstruction, sharp turns and edges contributed to the production of turbulence. Our initial ( $1^{\text {st }}$ generation) DAG redesign approach was to replace the vertical obstruction by a $20 \mathrm{deg}$-slanted (inclined) surface. CFD modeling results and preliminary test (performed with the revised DAG retrofitted into the baseline case) results as reported at the FMI Energy and Technical Services Conference in September 2002 indicated that this redesign would reduce turbulence intensity and ambient air infiltration. As shown in Figure 45, CFD modeling of the 20 deg-slanted surface design showed somewhat lower turbulence intensity on average compared to that of the baseline DAG configuration (actual geometry).

Based on these results, the $1^{\text {st }}$ generation DAG (air curtain) redesign was incorporated into the prototype case (see subsection 10.3 for detailed test results). Review of the prototype test results showed that the 20 deg-slanted surface created a sharp peak in the velocity profile at the outside of the case DAG (Figure 46). This gave a profile much different from the skewed parabolic profile sought, and resulted in a slight increase in infiltration load as compared to the baseline case.

After calibrating our CFD model using the prototype case test data, we further investigated the potential of altering the DAG geometry to obtain the desired velocity profile and reduce turbulence intensity. After more than 150 computer runs, a geometry with a widen throat and a 57 deg-slanted surface was identified. The reduced turbulence level for this proposed design can clearly be seen from Figure 45.

Figure 46 shows the velocity profile at the DAG for each case depicted in Figure 45 . Profiles for the baseline case DAG and the $20 \mathrm{deg}$-slanted surface of the prototype case are measured while that for the $57 \mathrm{deg}$-slanted surface is as computed by the model. It can be seen that the 57 deg-slanted surface with widened flow passage appears to generate the desired velocity profile. To demonstrate the effects these three geometries on the entrainment rate, we performed a series of parametric studies while imposing different turbulence intensities at the DAG. Figure 47 shows that at all turbulence intensities the 20 deg-slanted surface performed worse than the original design, however, the $57 \mathrm{deg}$-slanted surface design is projected to achieve a major improvement. Therefore, we can conclude that the shape of the velocity profile at the DAG is extremely important in controlling the entrainment rate along with turbulence intensity.

To demonstrate the impact of the throw angle at the DAG on the entrainment rate, several computer simulations were performed with the throw angle varying from 10 deg to 40 deg from the vertical position going in both directions, i.e., towards inside and outside of the display case. 


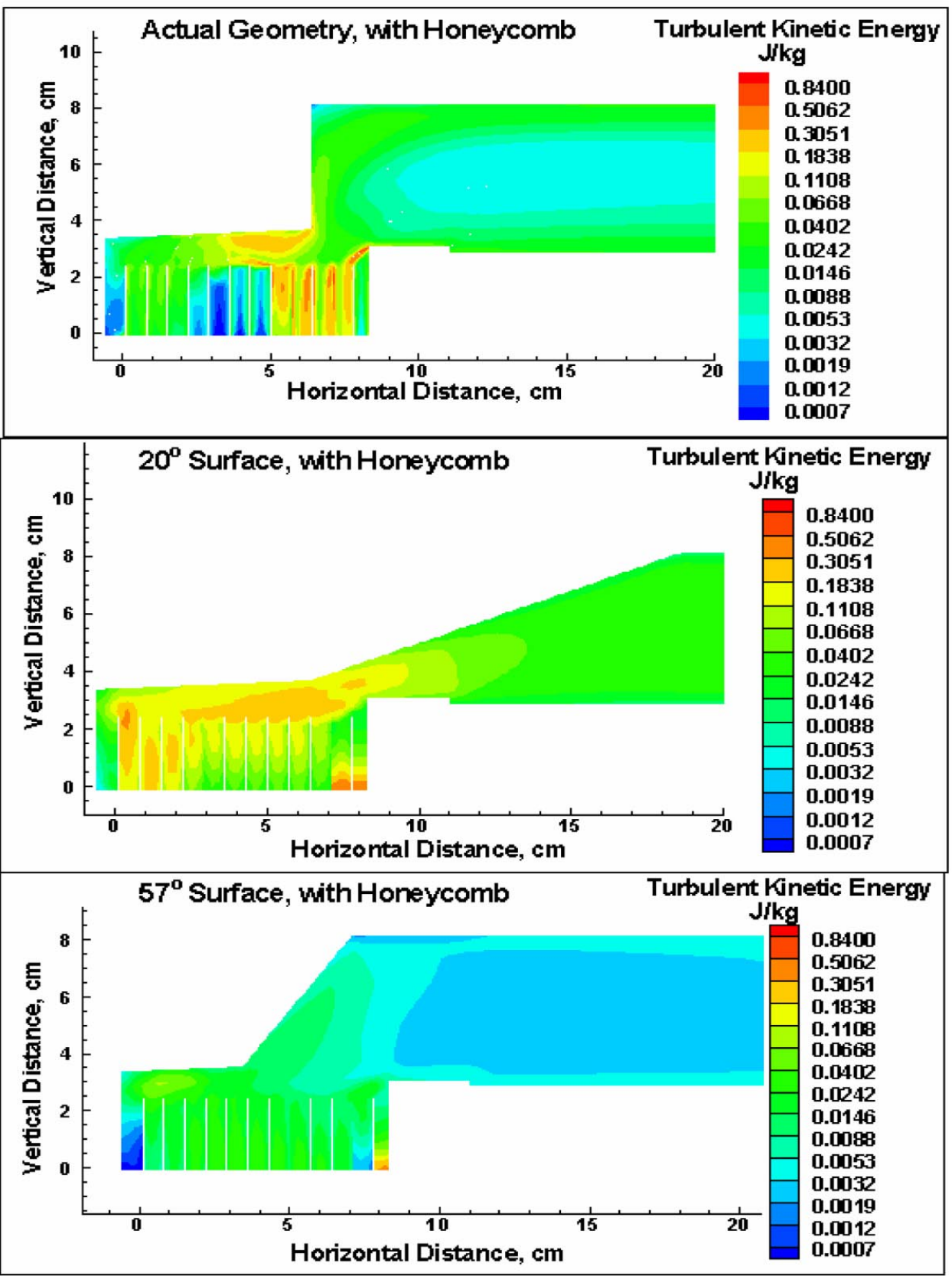

660-DORL-010231-36

Figure 45. Turbulent kinetic energy contours and velocity vectors for a variety of geometries at the DAG 


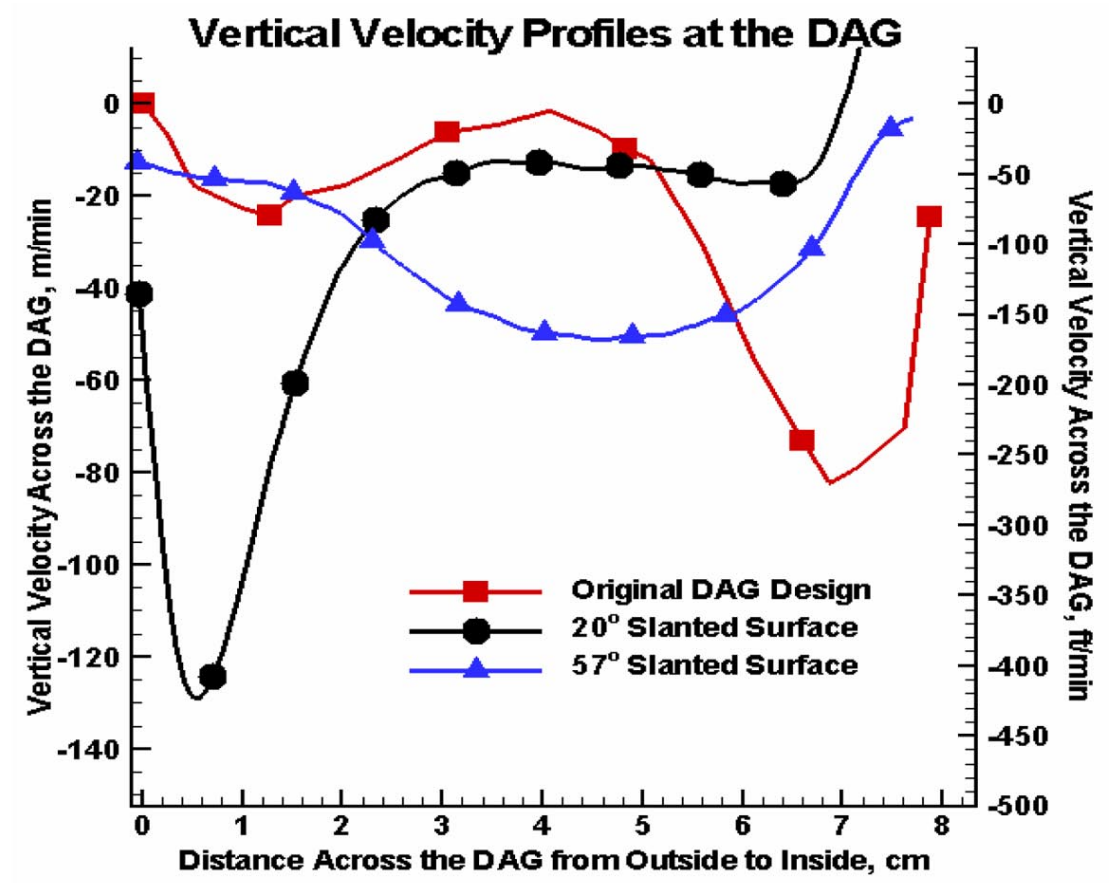

660-DORL-010231-37

Figure 46. Vertical velocity profile at the DAG for the all cases depicted in Figure 45 (NOTE: profiles from original baseline case DAG design and $20 \mathrm{deg}-$ slanted surface design of prototype case are from measured data; profile from 57 deg-slanted surface is estimated from CFD modeling)

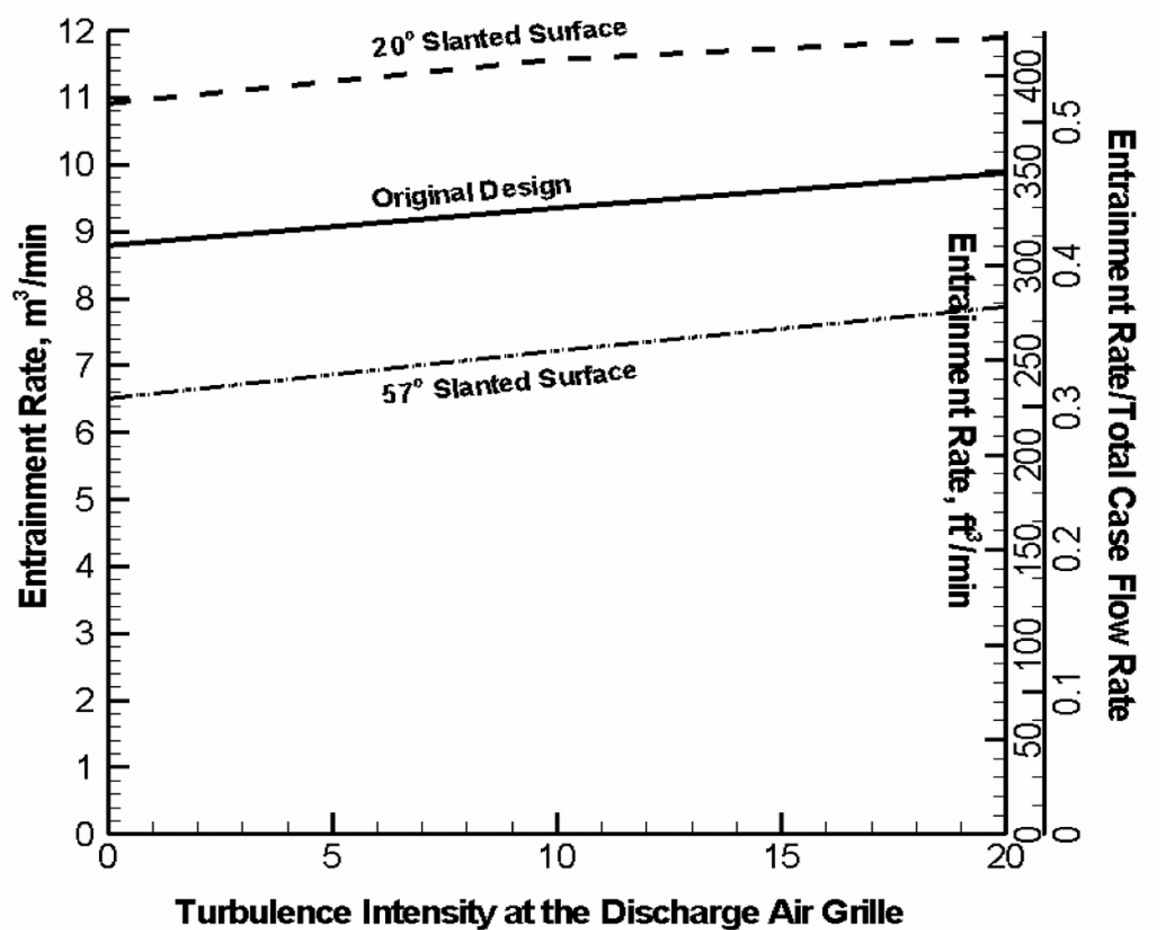

660-DORL-010231-38

Figure 47. Entrainment rate as a function of turbulence intensity at the DAG for different DAG designs 
The results are indicated in Figure 48. It seems that the entrainment rate reduces by about 12 percent (compared to the vertical discharge) at a throw angle of 14 deg towards the outside of the case. This could potentially reduce the infiltration rate by another 4 percent (about 30 percent of the total infiltration rate). When the throw angle is turned towards the back panel entrainment rate can be reduced by only about 3 percent (compared to the vertical discharge). This study shows that when the throw angle is pointing towards the outside of the display case at $12 \mathrm{deg}$ to $14 \mathrm{deg}$, some reduction in entrainment rate can be expected. The probable cause is that the curtain flow angle tends to push the warm ambient air away from the case so that it cannot participate in the mixing process.

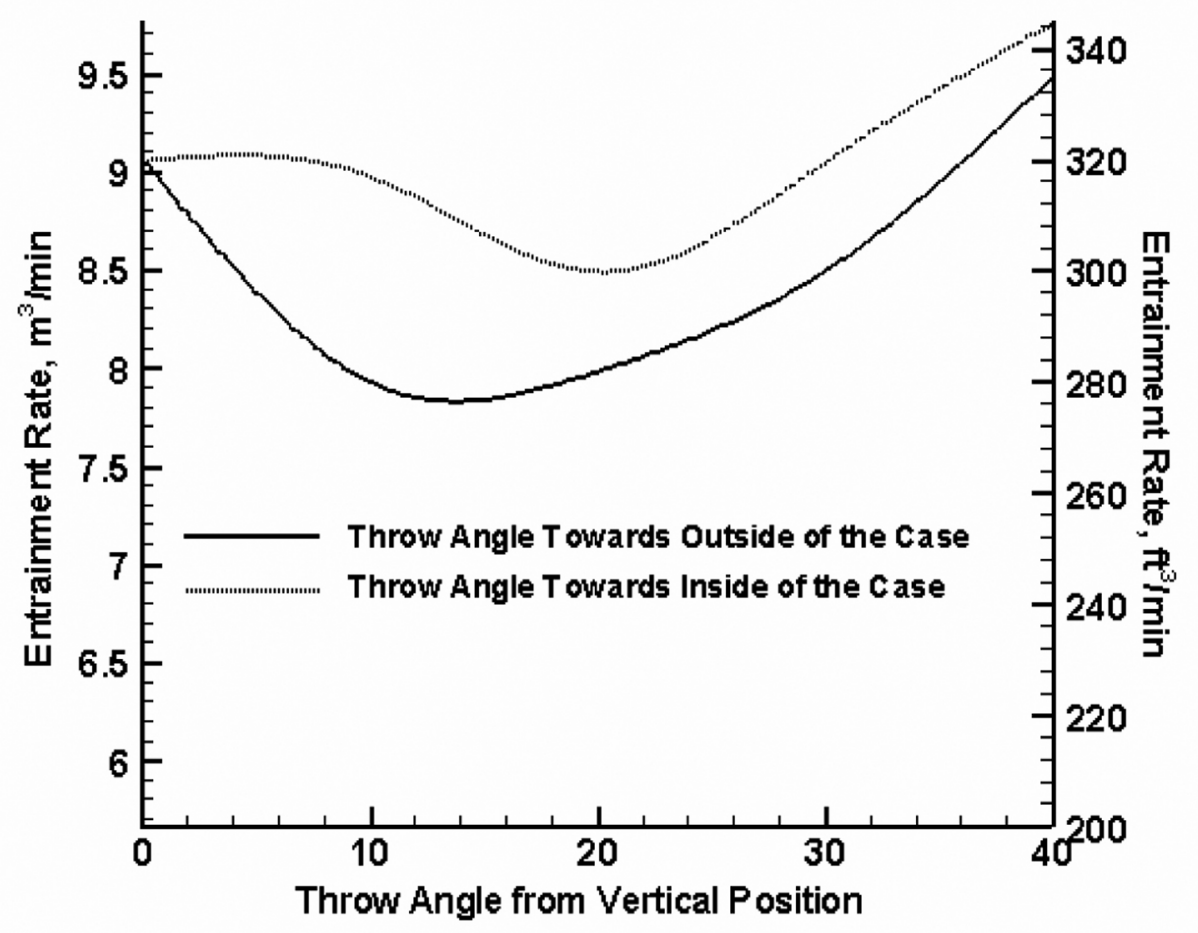

660-DORL-010231-39

Figure 48. Variation of entrainment rate with DAG throw angle 


\section{DISPLAY CASE TEST DATA THERMAL ANALYSIS APPROACH}

\subsection{Refrigeration Cycle Analysis}

A series of calculations were performed to obtain the key refrigeration parameters including the refrigeration load of the display case using refrigeration and psychrometric data. After the data was downloaded from the data logger and the data of interest was extracted, followed by preliminary reductions and calculations. These calculations included averaging of temperature, pressure, refrigerant mass flow, condensate weight, and discharge air velocities.

The total cooling load of the display case can be determined based on the refrigeration effect and mass flow rate of refrigerant. Determination of refrigeration effect and other quantities, such as superheat and sub-cooling quantities depend on the refrigerant enthalpies at specific locations within the refrigerant lines. Enthalpies can be either obtained from the refrigerant manufacturer's data at various temperatures and pressures, or calculated with respect to specific heat capacities and temperatures. In this analysis, some of the enthalpies were obtained from the AlliedSignal's refrigerant properties software, Genetron version R1.0, and some by calculation.

Once the temperatures and pressures were obtained, the enthalpies were determined. AlliedSignal's Genetron software was used to determine the saturated refrigerant temperatures as well as superheated refrigerant enthalpies. The data logger provided all pressures in pounds per square inch of gage (psig), which was used to obtain the saturated refrigerant temperatures and superheated enthalpies.

The enthalpies in the saturated phase were calculated using temperature-dependent expressions provided by DuPont, as well as using basic thermodynamic relationships. Equation 16 , provided by DuPont, was used to determine the saturated enthalpies (in $\mathrm{kJ} / \mathrm{kg}$ ) of refrigerant $404 \mathrm{~A}$ for a temperature range of $-20^{\circ} \mathrm{C}$ to $40^{\circ} \mathrm{C}$. The temperatures of the saturated liquid were first converted to Celsius, then inserted into Equation 16 to obtain the corresponding saturated enthalpies. The enthalpy of saturated liquid in the condenser was found using Equations 16 and 17.

$$
\begin{aligned}
& \mathrm{h}=200+1.438333 * \mathrm{~T}+0.003916667 * \mathrm{~T}^{2} \\
& \mathrm{~h}=\text { Enthalpy, }(\mathrm{kJ} / \mathrm{kg}) \\
& \mathrm{T}=\text { Temperature },\left({ }^{\circ} \mathrm{C}\right)
\end{aligned}
$$

In Equation 16, A, B, and C were constants, determined by DuPont, from the relationship between saturated enthalpy and temperature. Next, Equation 17 was used to convert the enthalpy 
in $\mathrm{kJ} / \mathrm{kg}$ to Btu/lb. Because of a change in reference states from SI to English units, a reference conversion factor, h (ref), was included in Equation 17.

$$
\begin{aligned}
& \mathrm{h}(\mathrm{Btu} / \mathrm{lb})=[\mathrm{h}(\mathrm{kJ} / \mathrm{kg})-\mathrm{h}(\mathrm{ref})] * 0.43021(\mathrm{Btu} / \mathrm{lb} / \mathrm{kJ} / \mathrm{kg}) \\
& \mathrm{h}(\mathrm{ref})=145.6 \mathrm{~kJ} / \mathrm{kg} \text { for } \mathrm{R} 404 \mathrm{~A}
\end{aligned}
$$

In order to determine the enthalpies for sub-cooled state, the thermodynamic relationship between enthalpy and temperature was incorporated. For this relationship, however, the correct liquid specific heat capacity was needed. Equation 18, provided by DuPont, establishes the specific heat capacity of liquid refrigerant $404 \mathrm{~A}$ for a temperature range of $-40^{\circ} \mathrm{F}$ to $140^{\circ} \mathrm{F}$. The net effect of system subcooling can be captured by comparing saturated liquid enthalpy and the final liquid enthalpy at the expansion valve inlet. The same approach was employed to determine the liquid line enthalpy at the inlet of the expansion valve.

$$
\begin{aligned}
& \mathrm{Cp}=0.306+4.083 \mathrm{E}-4 \mathrm{~T}-1.194 \mathrm{E}-6 \mathrm{~T}^{2}+8.056 \mathrm{E}-8 \mathrm{~T}^{3} \\
& \mathrm{Cp}=\text { Liquid heat capacity, }\left(\mathrm{Btu} / \mathrm{lb}{ }^{\circ} \mathrm{F}\right) \\
& \left.\mathrm{T}=\text { Average temp of the sub-cooled liquid (for a range of }-40^{\circ} \mathrm{F} \text { to } 140^{\circ} \mathrm{F}\right),\left({ }^{\circ} \mathrm{F}\right)
\end{aligned}
$$

Determination of the temperature difference between the SCT and the sub-cooled liquid entering the expansion valve $\left(\Delta \mathrm{T}_{\text {subcool }}\right)$ was required in order to determine the net sub-cooling effect of the system. The SCT (in ${ }^{\circ} \mathrm{F}$ ) was determined by using AlliedSignal's Genetron software based on discharge pressure (pressure at compressor outlet) data from the data acquisition system. Equation 19 was used to obtain the net sub-cooling effect $\left(\Delta \mathrm{T}_{\text {subcool }}\right)^{\text {' which }}$ is same as condenser sub-cooling.

$$
\begin{aligned}
\Delta \mathrm{T}_{\text {subcool }}= & \mathrm{SCT}-\mathrm{T}_{\text {subcool }} \\
\Delta \mathrm{T}_{\text {subcool }}= & \begin{array}{l}
\text { Temperature difference between } \mathrm{SCT} \text { and sub-cooled liquid entering the } \\
\text { expansion valve, }\left({ }^{\circ} \mathrm{F}\right)
\end{array} \\
\mathrm{T}_{\text {subcool }}= & \begin{array}{l}
\text { Average temperature of refrigerant at expansion valve inlet (value directly } \\
\text { extracted from the data acquisition system), }\left({ }^{\circ} \mathrm{F}\right)
\end{array}
\end{aligned}
$$

Next, the enthalpy change between the sub-cooled liquid and the saturated liquid was calculated by utilizing the following thermodynamic relationship.

$$
\Delta \mathrm{h}_{\text {subcool }}=\mathrm{Cp} * \Delta \mathrm{T}_{\text {subcool }}
$$
$\Delta \mathrm{h}_{\text {subcool }}=$ The enthalpy change between the sub-cooled liquid entering the expansion valve and saturated liquid in the condenser, (Btu/lb)

Finally, the enthalpy of the sub-cooled liquid was computed by subtracting the enthalpy change between the sub-cooled and saturated liquid from the enthalpy of saturated liquid (Equation 21). 
$\mathrm{h}_{\mathrm{ei}}=\mathrm{h}_{\text {satliq }}-\Delta \mathrm{h}_{\text {subcool }}$

$\mathrm{h}_{\mathrm{ei}} \quad=$ The sub-cooled liquid refrigerant enthalpy at the expansion valve inlet, (Btu/lb)

$\mathrm{h}_{\text {satliq }}=$ Saturated liquid enthalpy (value determined using Equations 16 and 17), (Btu/lb)

\subsubsection{Refrigeration Effect}

The refrigeration effect is the quantity of heat that each unit of mass of refrigerant absorbs to cool the refrigerated space. It simply represents the capacity of the evaporator per pound of refrigerant. This quantity was derived by subtracting the refrigerant enthalpy at the evaporator inlet (before the expansion valve) from the slightly superheated refrigerant enthalpy at the outlet of the evaporator (Equation 22).

$\mathrm{RE}=\mathrm{h}_{\mathrm{eo}}-\mathrm{h}_{\mathrm{ei}}$

$\mathrm{RE}=$ Refrigeration effect of the refrigerant in the evaporator, (Btu/lb)

$\mathrm{h}_{\mathrm{eo}}=$ Superheated refrigerant enthalpy at the evaporator exit (value determined by using the Genetron program), (Btu/lb)

\subsubsection{Refrigeration Load}

The refrigeration load of the case is the rate of cooling or heat removal (in Btu) that takes place at the evaporator of the display case per hour (Equation 23). This quantity is obtained by multiplying the refrigeration effect by refrigerant mass flow rate, which is extracted from the data acquisition system. The total case load for the display case was determined by using Equation 23.

$\dot{\mathrm{Q}}_{\mathrm{cr}}=\dot{\mathrm{m}}_{\mathrm{r}} * \mathrm{RE} * \mathrm{k}$

$\dot{\mathrm{Q}}_{\mathrm{cr}}=$ Total refrigeration load of the case, sensible and latent, (Btu/hr)

$\dot{\mathrm{m}}_{\mathrm{cr}}=$ Mass flow rate of refrigerant, (lb/min)

$\mathrm{k}=$ Conversion factor, $(60 \mathrm{~min} / \mathrm{hr})$

It is sometimes useful to determine the refrigeration load in tons. Thus, the refrigeration load of the case can be divided by 12,000, a conversion factor for Btu/hr to tons (Equation 24).

$$
\begin{aligned}
& \dot{\mathrm{Q}}_{\text {cr(tons) }}=\frac{\dot{\mathrm{Q}}_{\mathrm{cr}}}{12,000} \\
& \dot{\mathrm{Q}}_{\mathrm{cr}(\text { tons })}=\text { Refrigeration load, (tons) }
\end{aligned}
$$

In this project, the main constituents of the cooling load were determined, namely, sensible and latent. A key parameter required to define the latent load is the actual mass of water 
removed from the moist air as a result of refrigeration process. The data acquisition results revealed that this quantity was comprised of two components; mass of melted frost (at the end of defrost) and direct mass transfer of water from air during refrigeration. Frost formation is a direct result of the heat and mass transfer between moist air passing over the cold surface of the evaporator. Under high humidity conditions, a small portion of the moisture content of the air condensed and turned into droplets prior to forming frost crystalline on the coil. The data acquisition system provided the mass of water condensed from moist air during refrigeration. Additionally, under high humidity conditions a small portion of condensation took place during the defrost cycle. Although, during defrost, for some interval the refrigeration compressor did not operate, nevertheless, for a period of time the surface of the coil stayed below the dew point temperature of warm air passing through it. This resulted in some condensation from warm and moist air passing through the coil while the system was in defrost. This mass was determined by employing psychrometric analysis.

\subsubsection{Air Flow}

Psychrometric analysis relies heavily on the mass flow rate of the air within the thermodynamic boundary of the refrigerated fixture. The volume flow rate of air circulated throughout the fixture is a required parameter for conducting psychrometric calculations. This parameter was obtained using an approximation approach. This estimation relied on the discharge air velocity, free area available at the discharge air grill, and perforations in the back panel of the fixture (Equation 25).

$$
\begin{aligned}
& \operatorname{cfm}=\left(\mathrm{A}_{\text {back-panel }}+\mathrm{A}_{\mathrm{DAG}}\right) * \mathrm{DAV}_{\mathrm{avg}} \\
& \begin{array}{ll}
\mathrm{cfm} & =\text { Volumetric flow rate of air, }\left(\mathrm{ft}^{3} / \mathrm{min}\right) \\
\mathrm{A}_{\text {back-panel }} & =\text { Total area of openings in the back panel, }\left(\mathrm{ft}^{2}\right) \\
\mathrm{A}_{\mathrm{DAG}} & =\text { Total free area available through discharge air grill, }\left(\mathrm{ft}^{2}\right) \\
\mathrm{DAV}_{\mathrm{avg}} & =\text { Average discharge air velocity through discharge air grill, }(\mathrm{ft} / \mathrm{min})
\end{array}
\end{aligned}
$$

Once the volumetric flow rate of air into the display case was determined, the mass flow rate of air was obtained (Equation 26).

$$
\dot{\mathrm{m}}_{\mathrm{a}}=\mathrm{cfm} * \rho \mathrm{ai} * \mathrm{k}
$$

$\dot{\mathrm{m}}_{\mathrm{a}}=$ Mass flow rate of air, $(\mathrm{lb} / \mathrm{hr})$

$\rho_{\mathrm{ai}}=$ Density of air at the inlet of the evaporator coil, $\left(\mathrm{lb} / \mathrm{ft}^{3}\right)$

$\mathrm{k}=$ Conversion factor, $(60 \mathrm{~min} / \mathrm{hr})$

\subsubsection{Mass of Condensate}

Mass of condensate can be comprised of the following constituents:

- Mass of water vapor condensed from air during defrost period.

- Mass of water vapor condensed from air during refrigeration period. 
- $\quad$ Mass of melted frost during defrost.

The different components of condensate mass were obtained using the following equations. The total mass and the portion of condensate collected during refrigeration were obtained directly from scale readings. Equation 27 used psychrometric data to differentiate the defrost portion from the rest of condensate mass.

$$
\dot{\mathrm{m}}_{\mathrm{cd}}=\frac{[(\omega \mathrm{ai}-\omega \mathrm{ao}) * \dot{\mathrm{ma}} * \mathrm{td}]}{\mathrm{t}_{\mathrm{r}}}
$$

$\dot{\mathrm{m}}_{\mathrm{cd}}=$ Mass of water vapor condensed from air during defrost period, (lb/hr)

$\omega_{\mathrm{ai}}=$ Absolute humidity of air at the evaporator inlet, $\left(\mathrm{lb}_{\mathrm{w}} / \mathrm{lb}_{\mathrm{a}}\right)$

$\omega_{\mathrm{ao}}=$ Absolute humidity of air at the evaporator outlet, $\left(\mathrm{lb}_{\mathrm{w}} / \mathrm{lb} \mathrm{b}_{\mathrm{a}}\right)$

$\mathrm{t}_{\mathrm{d}} \quad=$ Defrost period, (hours)

$\mathrm{t}_{\mathrm{r}} \quad=$ Refrigeration period, (hours)

Finally, the mass of melted frost was determined by subtracting the sum of mass of water vapor condensed during the refrigeration and defrost periods from the total mass of collected condensate during total refrigeration run time (Equation 28).

$\dot{\mathrm{m}}_{\mathrm{f}}=\dot{\mathrm{m}}_{\mathrm{tc}}-\left(\dot{\mathrm{m}}_{\mathrm{cr}}+\dot{\mathrm{m}}_{\mathrm{cd}}\right)$

$\dot{\mathrm{m}}_{\mathrm{f}}=$ Mass of melted frost during defrost, (lb/hr)

$\dot{\mathrm{m}}_{\mathrm{tc}}=$ Total mass of condensate collected at the end of $24 \mathrm{hr}$ test period, (lb/hr)

$\dot{\mathrm{m}}_{\mathrm{cr}}=$ Mass of water vapor condensed from air during refrigeration period, $(\mathrm{lb} / \mathrm{hr})$

\subsubsection{Latent and Sensible Loads}

The latent load of the display case was calculated based on the mass of frost and mass of water vapor condensed from air during refrigeration period (Equation 29).

$$
\dot{\mathrm{Q}}_{\mathrm{lr}}=\left(\dot{\mathrm{m}}_{\mathrm{cr}}+\dot{\mathrm{m}}_{\mathrm{f}}\right) * \mathrm{~h}_{\mathrm{fg}}
$$

$\dot{\mathrm{Q}}_{\mathrm{lr}}=$ Latent load of refrigeration, (Btu/hr)

$\mathrm{h}_{\mathrm{fg}}=$ Heat of vaporization of water (based on average evaporator coil surface temperature), (Btu/lb)

Once the latent load was determined, the sensible load of the fixture was obtained by subtracting the latent load from the total refrigeration load (Equation 30).

$$
\dot{\mathrm{Q}}_{\mathrm{sr}}=\dot{\mathrm{Q}}_{\mathrm{cr}}-\dot{\mathrm{Q}}_{\mathrm{lr}}
$$




$$
\dot{\mathrm{Q}}_{\mathrm{sr}}=\text { Sensible load of refrigeration, (Btu/hr) }
$$

\subsubsection{Cooling Load Based on One Running Cycle}

Based on ASHRAE Standard 72-98, the cooling load of the display case must be determined from one running cycle of data within the test. Running cycle refers to the refrigeration period between two defrost periods. This calculation is primarily based on refrigerant properties during the last 3/4 th's of the running cycle (based on ASHRAE Standard 72-98). Equation 31 is used to calculate the cooling load during last $3 / 4$ th's of the running cycle.

$$
\dot{\mathrm{Q}}_{\mathrm{rt}}=\frac{\left(\mathrm{h}_{\mathrm{v}}-\mathrm{h}_{1}\right)}{\mathrm{t}_{\mathrm{rt}}} * \mathrm{~m}_{\mathrm{rt}}
$$

$\dot{\mathrm{Q}}_{\mathrm{rt}}=$ Average cooling load for the running cycle, $(\mathrm{Btu} / \mathrm{hr})$

$\mathrm{h}_{\mathrm{v}}=$ Enthalpy of leaving refrigerant vapor during the last $3 / 4$ of the running cycle, (Btu/lb)

$\mathrm{h}_{1}=$ Enthalpy of entering liquid refrigerant during the entire running cycle, $(\mathrm{Btu} / \mathrm{lb})$

$\mathrm{m}_{\mathrm{rt}}=$ Total refrigerant mass flow for the running cycle, $(\mathrm{lb})$

$\mathrm{t}_{\mathrm{rt}}=$ Refrigeration time period for the running cycle, $(\mathrm{hr})$

The reduction factor is the ratio of refrigeration time period for the running cycle to overall time for one running cycle plus one defrost period (Equation 32). Multiplying the resulting reduction factor by the average cooling load for the running cycle will result to arrive at a reduced average cooling load for the overall time period (Equation 33).

$$
\mathrm{RF}=\frac{\mathrm{t}_{\mathrm{rt}}}{\mathrm{t}_{\mathrm{oa}}}
$$

$\mathrm{RF}=$ Reduction factor, (unit-less)

$\mathrm{t}_{\mathrm{oa}}=$ Overall time for one running cycle plus one defrost period, (hr)

$$
\dot{\mathrm{Q}}_{\mathrm{oa}}=\dot{\mathrm{Q}}_{\mathrm{rt}} * \mathrm{RF}
$$

$\dot{\mathrm{Q}}_{\mathrm{oa}}=$ Reduced average cooling load for the overall time period, (Btu/hr)

\subsubsection{Cooling Load Using Psychrometric Data}

To gain confidence in the obtained refrigeration load, the cooling load was also calculated based on psychrometric data. Using psychrometric data, the total case cooling load of the fixture, including frost loads, was determined by using Equation 34. The latent and sensible loads were determined by using Equations 35 and 36, respectively. 
$\dot{\mathrm{Q}}_{\mathrm{ca}}=\dot{\mathrm{m}}_{\mathrm{a}} *\left(\mathrm{~h}_{\mathrm{ai}}-\mathrm{h}_{\mathrm{ao}}\right)+\left\{\dot{\mathrm{m}}_{\mathrm{f}} *\left[\mathrm{Cp}_{\mathrm{w}} *\left(\mathrm{~T}_{\mathrm{dp}}-\mathrm{T}_{\mathrm{f}}\right)+\mathrm{h}_{\mathrm{f}}+\mathrm{Cp}_{\mathrm{i}} *\left(\mathrm{~T}_{\mathrm{f}}-\mathrm{T}_{\mathrm{i}}\right)\right]\right\}$

$\dot{\mathrm{Q}}_{\mathrm{ca}}=$ Total refrigeration load using psychrometric data, (Btu/hr)

$\mathrm{h}_{\mathrm{ao}}=$ Enthalpy of exiting air at evaporator coil, (Btu/lb)

$\mathrm{h}_{\mathrm{ai}}=$ Enthalpy of entering air at evaporator coil, (Btu/lb)

$\mathrm{Cp}_{\mathrm{w}}=$ Specific heat of water, $\left(\sim 1.0 \mathrm{Btu} / \mathrm{lb}-{ }^{\circ} \mathrm{F}\right)$

$\mathrm{T}_{\mathrm{dp}}=$ Dew point temperature of air at coil surface temperature, $\left({ }^{\circ} \mathrm{F}\right)$

$\mathrm{T}_{\mathrm{f}} \quad=$ Freezing temperature of water, $\left(32^{\circ} \mathrm{F}\right)$

$\mathrm{h}_{\mathrm{f}}=$ Heat of fusion of ice, $(143.5 \mathrm{Btu} / \mathrm{lb})$

$\mathrm{Cp}_{\mathrm{i}}=$ Specific heat capacity of ice, $\left(0.487 \mathrm{Btu} / \mathrm{lb}-{ }^{\circ} \mathrm{F}\right)$

$\mathrm{T}_{\mathrm{i}}=$ Average ice temperature on surface of evaporator coil, $\left({ }^{\circ} \mathrm{F}\right)$

$\dot{\mathrm{Q}}_{\mathrm{la}}=\dot{\mathrm{m}}_{\mathrm{a}} *\left(\omega_{\mathrm{ai}}-\omega_{\mathrm{ao}}\right) * \mathrm{~h}_{\mathrm{fg}}$

$\dot{\mathrm{Q}}_{\mathrm{la}}=$ Latent load of refrigeration using psychrometric data, (Btu/hr)

$\dot{\mathrm{Q}}_{\mathrm{sa}}=\dot{\mathrm{Q}}_{\mathrm{ca}}-\dot{\mathrm{Q}}_{\mathrm{la}}$

$\dot{\mathrm{Q}}_{\mathrm{sa}}=$ Sensible load of refrigeration using psychrometric data, (Btu/hr)

\subsubsection{Evaporator Coil Characteristic Performance}

One indication of coil performance is the temperature differential across the evaporator coil. The temperature differential across the evaporator coil was determined based on measured air temperatures at the inlet and outlet of the evaporator coil, Equation 37.

$\Delta \mathrm{T}_{\mathrm{e}}=\mathrm{T}_{\mathrm{ai}}-\mathrm{T}_{\mathrm{ao}}$

$\Delta \mathrm{T}_{\mathrm{e}}=$ Temperature differential across the evaporator coil, $\left({ }^{\circ} \mathrm{F}\right)$

$\mathrm{T}_{\mathrm{ai}}=$ Temperature of air entering the evaporator coil, $\left({ }^{\circ} \mathrm{F}\right)$

$\mathrm{T}_{\mathrm{ao}}=$ Temperature of air leaving the evaporator coil, $\left({ }^{\circ} \mathrm{F}\right)$

Another indication of coil performance is the evaporator TD. It is defined as the difference in temperature between the temperature of the air leaving the evaporator and the saturation temperature of the refrigerant corresponding to the pressure at the evaporator coil outlet (Equation 38). The saturation temperature at the outlet of the evaporator coil was determined using AlliedSignal's Genetron program.

$\mathrm{TD}_{\mathrm{e}}=\mathrm{T}_{\mathrm{ao}}-\mathrm{SET}$

$\mathrm{TD}_{\mathrm{e}}=$ Evaporator $\mathrm{TD},\left({ }^{\circ} \mathrm{F}\right)$

SET $=$ Saturated evaporator temperature based on evaporator coil outlet pressure, $\left({ }^{\circ} \mathrm{F}\right)$ 
The evaporator coil superheat, which is one of the system parameters, was determined based on vapor refrigerant temperature at the outlet of the evaporator coil and the saturation temperature of the refrigerant corresponding to the pressure at the outlet of the evaporator coil according to Equation 39.

$$
\begin{aligned}
& \mathrm{SH}_{\mathrm{e}}=\mathrm{T}_{\mathrm{v}}-\mathrm{SET} \\
& \mathrm{SH}_{\mathrm{e}}=\text { Evaporator coil superheat, }\left({ }^{\circ} \mathrm{F}\right) \\
& \mathrm{T}_{\mathrm{v}}=\text { Vapor refrigerant temperature at the outlet of the evaporator coil, }\left({ }^{\circ} \mathrm{F}\right)
\end{aligned}
$$

One of the important indications of the evaporator coil's performance is sensible heat ratio (SHR). It indicates the amount of sensible cooling being done on the air as opposed to the total cooling, including the amount of moisture removal. The evaporator coil SHR can be determined by the ratio of sensible load to total refrigeration load of the fixture (Equation 40).

$$
\mathrm{SHR}=\frac{\dot{\mathrm{Q}}_{\mathrm{sr}}}{\dot{\mathrm{Q}}_{\mathrm{cr}}}
$$

SHR $=$ Sensible heat ratio of the evaporator

Another important indication of coil performance is the ability of the coil to remove moisture from the air. This value is determined by multiplying the mass flow of air through the coil by the difference between the air absolute humidity at the coil inlet and outlet (Equation 41).

$$
\operatorname{MRR}=\dot{\mathrm{m}}_{\mathrm{a}} *\left(\omega_{\mathrm{ai}}-\omega_{\mathrm{ao}}\right) * \mathrm{k}
$$

$\mathrm{MRR}=$ Moisture removal rate of the evaporator, $(\mathrm{lb} / \mathrm{hr})$

$\mathrm{k}=$ Conversion factor, $(60 \mathrm{~min} / \mathrm{hr})$

The evaporator heat exchange effectiveness is dependent on its log-mean temperature difference (LMTD) and its effective overall heat transfer coefficient, UA. The LMTD is determined using the refrigerant and air temperatures at the inlet and outlet of the evaporator coil according to Equation 42.

$$
\operatorname{LMTD}=\frac{\left(\mathrm{T}_{\mathrm{ai}}-\mathrm{T}_{\mathrm{ao}}\right)}{\left[\ln \left(\frac{\mathrm{T}_{\mathrm{ai}}-\mathrm{SET}}{\mathrm{T}_{\mathrm{ao}}-\mathrm{SET}}\right)\right]}
$$

LMTD = Evaporator coil log-mean temperature difference, ${ }^{\circ} \mathrm{F}$

Once the evaporator coil LMTD was determined, the effective overall heat transfer coefficient, UA, of the coil can be determined by the ratio of total refrigeration load to the coil 
LMTD (Equation 43). The UA of the evaporator coil is a function of coil material and its effective surface area.

$$
\mathrm{UA}=\frac{\dot{\mathrm{Q}}_{\mathrm{cr}}}{\mathrm{LMTD}}
$$

$\mathrm{UA}=$ Effective overall heat transfer coefficient of the coil, $\left(\mathrm{Btu} / \mathrm{hr}-{ }^{\circ} \mathrm{F}\right)$

\subsubsection{Heat of Compression}

Heat of compression is an important parameter for calculating the amount of heat rejection at the condenser and calculating the theoretical compressor power. In order to obtain the heat of compression, the mass flow rate of refrigerant was multiplied by the difference between the enthalpies of refrigerant at the compressor inlet and discharge (Equation 44).

$$
\dot{\mathrm{Q}}_{\mathrm{c}}=\dot{\mathrm{m}}_{\mathrm{r}} *\left(\mathrm{~h}_{\mathrm{co}}-\mathrm{h}_{\mathrm{ci}}\right) * \mathrm{k}
$$

$\dot{\mathrm{Q}}_{\mathrm{c}}=$ Total (over test period) heat of compression, (Btu/hr)

$\mathrm{h}_{\mathrm{co}}=$ Superheated enthalpy at the outlet of the compressor (value determined by using the Genetron program), (Btu/lb)

$\mathrm{h}_{\mathrm{ci}}=$ Superheated enthalpy at the inlet to the compressor (value determined by using the Genetron program), (Btu/lb)

$\mathrm{k}=$ Conversion factor, $60,(\mathrm{~min} / \mathrm{hr})$

The compressor power was normalized based on average compressor efficiency. An estimation approach was utilized to determine the average compressor efficiency. The actual amount of power supplied to the compressor was recorded by the data acquisition system. This data along with calculated heat of compression was used to obtain the compressor efficiency according to Equation 45.

$$
\eta=\frac{\dot{\mathrm{Q}}_{\mathrm{c}}}{\left(\mathrm{kW}_{\mathrm{c}} * \mathrm{~K}\right)}
$$

$\eta \quad=$ Actual compressor efficiency represented for each test scenario

$\mathrm{kW}_{\mathrm{c}}=$ Average power supplied to the compressor (recorded by the data acquisition system), (kW)

$\mathrm{K}=$ Conversion factor, $(3,413 \mathrm{Btu} / \mathrm{hr} / \mathrm{kW})$

Once a representative compressor efficiency was determined for each test scenario, the values were then averaged to obtain an overall efficiency (Equation 46). 


$$
\eta_{\text {overall }}=\frac{\sum \eta}{n}
$$

$\eta_{\text {overall }}=$ Overall average efficiency of compressor

$\mathrm{n} \quad=$ Number of test scenarios

After determining the overall average efficiency of the compressor, the compressor power was normalized using Equation 47.

$$
\begin{aligned}
& \mathrm{kW}_{\text {norm }}=\frac{\dot{\mathrm{Q}}_{\mathrm{c}}}{\left(\eta_{\text {overall }} * \mathrm{~K}\right)} \\
& \mathrm{kW}_{\text {norm }}=\text { Normalized compressor power, }(\mathrm{kW}) \\
& \mathrm{K} \quad=\text { Conversion factor, }(3,413 \mathrm{Btu} / \mathrm{hr} / \mathrm{kW})
\end{aligned}
$$

\subsubsection{Total System Power and Energy}

Total system power and energy use for this test excluded condenser power. Total system power for the fixture was obtained using Equation 48.

$$
\begin{aligned}
& \mathrm{kW}_{\text {Total }}=\mathrm{kW}_{\text {evap-fans }}+\mathrm{kW}_{\text {lights }}+\mathrm{kW}_{\mathrm{ASH}}+\mathrm{kW}_{\text {norm }} \\
& \begin{array}{ll}
= & \text { Power consumed by the refrigeration system not including the condenser, } \\
\mathrm{kW}_{\text {Total }} & (\mathrm{kW}) \\
\mathrm{kW}_{\text {evap-fans }}= & \text { Power consumed by the evaporator fan motors, }(\mathrm{kW}) \\
\mathrm{kW}_{\text {lights }}= & \text { Power consumed by the light fixtures in the case, }(\mathrm{kW}) \\
\mathrm{kW}_{\mathrm{ASH}}= & \text { Power consumed by the anti-sweat heaters, }(\mathrm{kW})
\end{array}
\end{aligned}
$$

The energy consumption of the lights, evaporator fan motors, compressor, and anti-sweat heaters (ASH) is defined as the product of supplied power and total hours of power usage. Lights, evaporator fan motors, and ASH stayed on continuously, hence, their total hours of power usage was equal to the hours of total test (Equations 49, 50 and 51). The hours of power usage for the compressor was less than total test hours, since the compressor did not run during the defrost periods (Equation 52).

$$
\begin{aligned}
& \mathrm{kWh}_{\text {evap-fans }}=\mathrm{kW}_{\text {evap-fans }} * \mathrm{t}_{\text {evap-fans }} \\
& \mathrm{kWh}_{\text {evap-fans }}=\text { Energy consumed by the evaporator fan motors, (kWh) } \\
& \mathrm{t}_{\text {evap-fans }} \quad=\text { Total time of power usage by evaporator fan motors, (hours) } \\
& \mathrm{kWh}_{\text {lights }}=\mathrm{kW}_{\text {lights }} * \mathrm{t}_{\text {lights }} \\
& \mathrm{kWh}_{\text {lights }}=\text { Energy consumed by the lights in the case, }(\mathrm{kWh}) \\
& \mathrm{t}_{\text {lights }}=\text { Total time of power usage by lights, (hours) }
\end{aligned}
$$


$\mathrm{kWh}_{\mathrm{ASH}}=\mathrm{kW}_{\mathrm{ASH}} * \mathrm{t}_{\mathrm{ASH}}$

$\mathrm{kWh}_{\mathrm{ASH}}=$ Energy consumed by the ASH, $(\mathrm{kWh})$

$\mathrm{t}_{\mathrm{ASH}}=$ Total time of power usage by ASH, (hours)

$\mathrm{kWh}_{\text {norm }}=\mathrm{kW}_{\text {norm }} * \mathrm{t}_{\mathrm{c}}$

$\mathrm{kWh}_{\text {norm }}=$ Energy consumed by the compressor, $(\mathrm{kWh})$

$\mathrm{t}_{\mathrm{c}} \quad=$ Total time of power usage by the compressor, (hours)

Once energy consumed by each individual component was determined, then the total energy consumption for the fixture over entire test period was obtained by using Equation 53.

$\mathrm{kWh}_{\text {Total }}=\mathrm{kWh}_{\text {evap-fans }}+\mathrm{kWh}_{\text {lights }}+\mathrm{kWh}_{\mathrm{ASH}}+\mathrm{kWh}_{\text {norm }}$

$\mathrm{kWh}_{\text {Total }}=$ Energy consumed by the refrigeration system not including the condenser, $(\mathrm{kWh})$

\subsection{Display Case Heat Transfer Analysis}

The heat transfer within a display case involves interactions between the product and the internal environment of the case, as well as incoming heat from the surroundings into the case. The constituents of incoming heat from the surrounding environment include transmission (or conduction), infiltration and radiation. The heat from the internal sources include anti-sweat heaters, lighting and evaporator fan motor(s).

Conduction and radiation loads depend on the temperatures within the case and that of ambient air. Open display cases rely on the effectiveness of their air curtains to prevent the penetration of warm and moist ambient air into the cold environment inside the case. Air curtain plays a significant role in thermal interaction of a vertical display case and surrounding ambient air. The following sections provide a detail discussion of the display case cooling load components as well as methodologies employed in this project to quantify them.

\subsubsection{Transmission (or Conduction) Load}

The transmission load refers to the conduction of heat through the display case shell. The temperature difference between the air in the room and the inside surfaces of the case is the driving force for this transfer of heat. The first task in determining the transmission load was to determine the overall coefficient of heat transfer of the case walls. This involves determination of all outside and inside air film convective coefficients, thermal conductivity of the outer and inner walls of the case, and thermal conductivity of the insulation between the inner and outer walls. A simplified schematic of display case's wall assembly layers is shown in Figure 49. Equation 54 describes the approach used to determine the overall coefficient of heat transfer for the display case. 


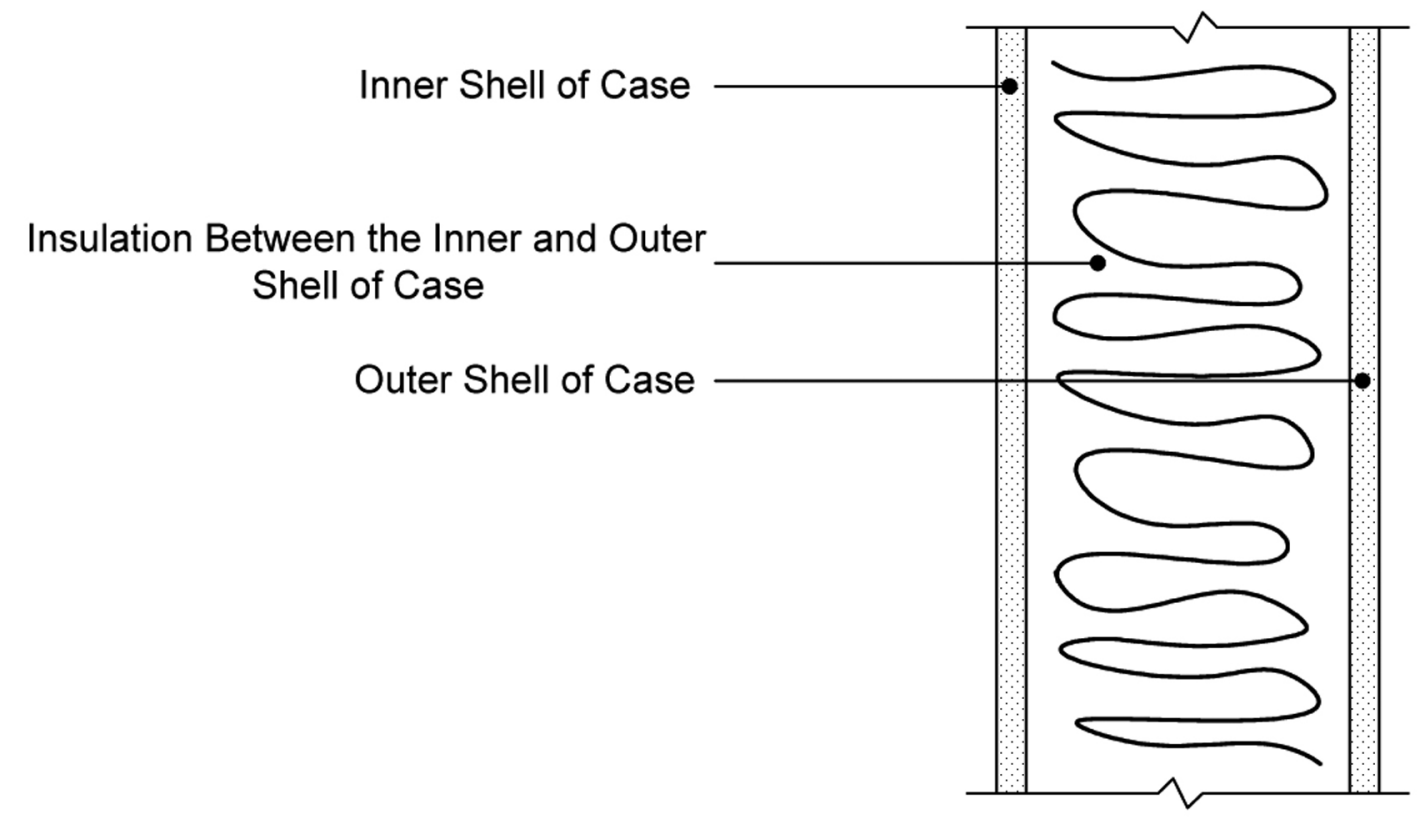

660-DORL-010231-40

Figure 49. Schematics of inner and outer shell of the case and insulation between them

$$
\mathrm{U}=\frac{1}{\left[\left(\frac{1}{\mathrm{~h}_{\mathrm{i}}}\right)+\left(\frac{\mathrm{L}_{1}}{\mathrm{k}_{1}}\right)+\left(\frac{\mathrm{L}_{2}}{\mathrm{k}_{2}}\right)+\left(\frac{\mathrm{L}_{3}}{\mathrm{k}_{3}}\right)+\left(\frac{1}{\mathrm{~h}_{\mathrm{o}}}\right)\right]}
$$

$\mathrm{U}=$ Overall coefficient of heat transfer coefficient for the case walls, $\left(\mathrm{Btu} / \mathrm{hr}-\mathrm{ft}^{2}{ }^{\circ} \mathrm{F}\right)$

$\mathrm{h}_{\mathrm{i}}=$ Convective coefficient for inside case air film against case inner wall, $\left(\mathrm{Btu} / \mathrm{hr}-\mathrm{ft}^{2}{ }^{\circ} \mathrm{F}\right)$

$\mathrm{L}_{1}=$ Thickness of outer shell of the case, (in.)

$\mathrm{k}_{1}=$ Thermal conductivity of outer shell of case, $\left(\mathrm{Btu}-\mathrm{in} . / \mathrm{hr}-\mathrm{ft}^{2}-{ }^{\circ} \mathrm{F}\right)$

$\mathrm{L}_{2}=$ Thickness of insulation within the case walls, (in.)

$\mathrm{k}_{2}=$ Thermal conductivity of insulation within the case walls, (Btu-in./hr- $\mathrm{ft}^{2}-{ }^{\circ} \mathrm{F}$ )

$\mathrm{L}_{3}=$ Thickness of inner shell of the case, (in.)

$\mathrm{k}_{3}=$ Thermal conductivity of inner shell of case, (Btu-in./hr- $\left.\mathrm{ft}^{2}-{ }^{\circ} \mathrm{F}\right)$

$\mathrm{h}_{\mathrm{O}}=$ Convective coefficient for outside/room air film against case outer shell, $\left(\mathrm{Btu} / \mathrm{hr}-\mathrm{ft}^{2}-{ }^{\circ} \mathrm{F}\right)$

Once the overall coefficient of heat transfer was determined, the transmission load was determined using Equation 55. The inside temperature of various surfaces inside the case was assumed to be in equilibrium with the air temperature inside the case. 
$\dot{\mathrm{Q}}_{\text {cond }}=\mathrm{U} * \mathrm{~A} *\left(\mathrm{~T}_{\text {room }-} \mathrm{T}_{\text {case }}\right)$

$\dot{\mathrm{Q}}_{\text {cond }}=$ Transmission, or conduction, load of the case, $(\mathrm{Btu} / \mathrm{hr})$

$\mathrm{A}=$ Total surface area of case walls that are conducting heat, $\left(\mathrm{ft}^{2}\right)$

$\mathrm{T}_{\text {room }}=$ Dry bulb temperature of the air in the room, $\left({ }^{\circ} \mathrm{F}\right)$

$\mathrm{T}_{\text {case }}=$ Dry bulb temperature of the air inside the display case, $\left({ }^{\circ} \mathrm{F}\right)$

\subsubsection{Radiation Load}

The temperature of walls inside the controlled environment room was assumed to be in equilibrium with the temperature of air inside the room. Similar to the conduction analysis, the inside temperature of various surfaces inside the case was assumed to be in equilibrium with the air temperature inside the case. This assumption was later verified and accepted once the subject temperatures were measured individually and were found to be in equilibrium with the air temperature adjacent to them. The case load due to radiation heat transfer was determined by simply modeling the system as two gray surfaces, one surface representing the total surface area of the room (walls, floor, ceiling), and the other being an imaginary plane covering the opening of the display case. All of the radiation leaving the room surfaces will arrive at the imaginary plane. The imaginary plane at the case opening will, in turn, exchange all of its radiation with the interior surfaces of the display case. A series of calculations were performed to develop the effective view factor between the room and inside of the case using Kirchoff's Law and the reciprocity relation.

Figure 50 shows a simplified, plan view of the controlled environment room and the surfaces exchanging heat through radiation with the display case. The surfaces inside of the display case (back, top, bottom, and sides) were all designated by surface 1, the room surfaces were designated by surface 2 , and the imaginary plane covering the case opening was designated by surface 3. From the reciprocity relation, $A_{1} F_{1-3}=A_{3} F_{3-1}$. In this case, $F_{3-1}$ is 1 , and $F_{1-3}=F_{1-2}$, therefore, $F_{1-2}=A_{3} / A_{1}$. Once this view factor was determined, equation 56 was used to calculate the radiation load of the cases.

$$
\begin{aligned}
& \dot{\mathrm{Q}}_{\mathrm{rad}}=\frac{\sigma *\left(\mathrm{~T}_{\mathrm{w}}{ }^{4}-\mathrm{T}_{\mathrm{c}}{ }^{4}\right)}{\left[\left(\frac{1-\varepsilon_{\mathrm{w}}}{\varepsilon_{\mathrm{w}} * \mathrm{~A}_{\mathrm{w}}}\right)+\left(\frac{1}{\mathrm{~A}_{\mathrm{w}} * \mathrm{~F}_{\mathrm{cw}}}\right)+\left(\frac{1-\varepsilon_{\mathrm{w}}}{\varepsilon_{\mathrm{c}} * \mathrm{~A}_{\mathrm{c}}}\right)\right]} \\
& \dot{\mathrm{Q}}_{\mathrm{rad}}=\text { Radiation heat transfer between room walls and display case, }(\mathrm{Btu} / \mathrm{hr}) \\
& \sigma \quad=\text { Stefan-Boltzmann Constant, }\left(0.1714 * 10^{-8} \mathrm{Btu} / \mathrm{hr}-\mathrm{ft}^{2}-\mathrm{R}^{4}\right) \\
& \mathrm{T}_{\mathrm{w}}=\text { Surface temperature of the room walls, }(\mathrm{R}) \\
& \mathrm{T}_{\mathrm{c}}=\text { Surface temperature of the display case inner walls, }(\mathrm{R}) \\
& \varepsilon_{\mathrm{w}}=\text { Emissivity of the room walls } \\
& \mathrm{A}_{\mathrm{w}}=\text { Total area of room surfaces, }\left(\mathrm{ft}^{2}\right) \\
& \mathrm{F}_{\mathrm{cw}}=\text { View factor from case to surfaces of the room }
\end{aligned}
$$




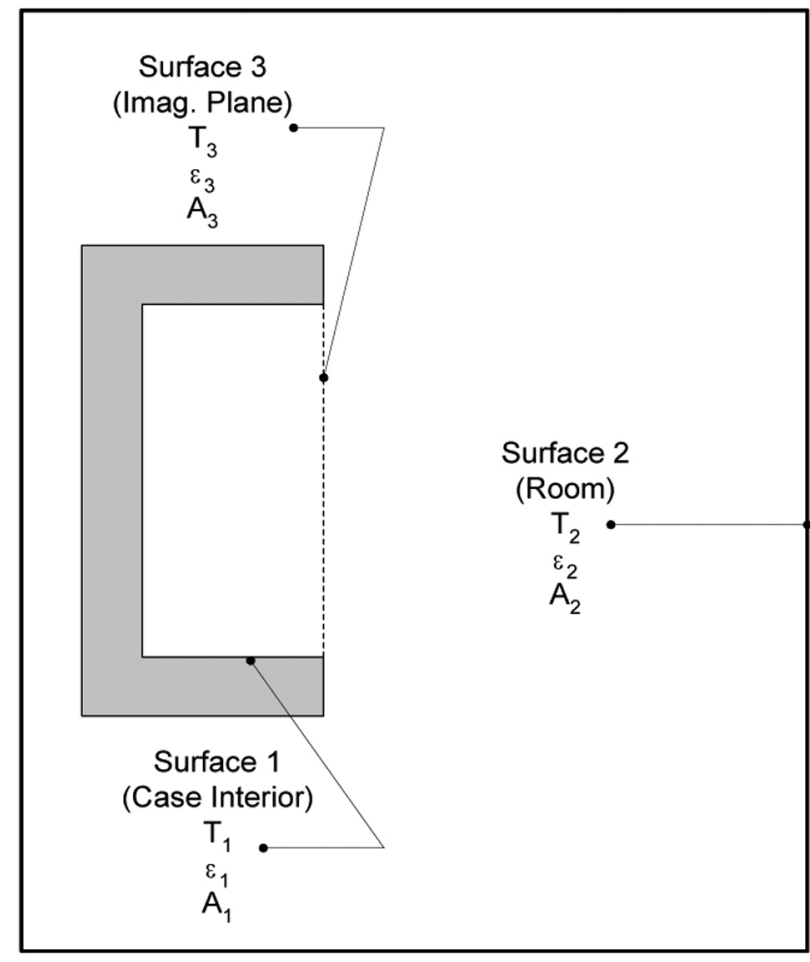

660-DORL-010231-41

Figure 50. Surfaces participating in radiation heat transfer for display case

$\varepsilon_{\mathrm{c}} \quad=$ Emissivity of the inside walls of the case

$\mathrm{A}_{\mathrm{c}}=$ Total area of the inside walls of the case, $\left(\mathrm{ft}^{2}\right)$

\subsubsection{Internal Load}

The internal load of the display case refers to the heat introduced and dissipated by its internal components. The internal load for the display case under consideration include the heat introduced by the case lighting and by the evaporator fan motors. The fan motors, lamps, and ballasts are located inside the thermodynamic boundary of the case. Hence, their total heat dissipation is considered part of the case load. The power consumed by these devices was recorded directly by the data logger, which was then converted to cooling load according to Equations 57 and 58.

$\dot{\mathrm{Q}}_{\text {evap }}=\mathrm{kW}_{\text {evap-fans }} * \mathrm{~K}$

$\dot{\mathrm{Q}}_{\text {lights }}=\mathrm{kW}_{\text {lights }} * \mathrm{~K}$

$\dot{\mathrm{Q}}_{\text {evap }} \quad=$ Case load due to fan motors, $(\mathrm{Btu} / \mathrm{hr})$

$\dot{\mathrm{Q}}_{\text {lights }}=$ Case load due to lighting, $(\mathrm{Btu} / \mathrm{hr})$

$\mathrm{kW}_{\text {evap-fans }}=$ Power consumed by the fan motors, $(\mathrm{kW})$ 
$\mathrm{kW}_{\text {lights }} \quad=$ Power consumed by the light fixtures in the case, $(\mathrm{kW})$

$\mathrm{K}=$ Conversion factor, $(3,413 \mathrm{Btu} / \mathrm{hr} / \mathrm{kW})$

\subsubsection{Infiltration Load}

The infiltration load of the display case refers to the entrainment of warm and moist air from the room, across the case air curtain, into the refrigerated space. The infiltration load has two components-sensible and latent. The sensible portion refers to the temperature driven heat penetrating into the display case, whereas the latent portion refers to the heat content of moisture within the infiltrating air. As air passes through the evaporator, it loses its sensible heat and dehumidifies as well.

A reverse calculation approach was used to determine the infiltration load of the display cases. Upon obtaining the total case load along with all other cooling components, Equation 59 was used to obtain the total infiltration load.

$$
\dot{\mathrm{Q}}_{\text {inf }}=\dot{\mathrm{Q}}_{\mathrm{cr}}-\left[\dot{\mathrm{Q}}_{\mathrm{evap}}+\dot{\mathrm{Q}}_{\text {lights }}+\dot{\mathrm{Q}}_{\mathrm{ASH}}+\dot{\mathrm{Q}}_{\mathrm{cond}}+\dot{\mathrm{Q}}_{\mathrm{rad}}\right]
$$

$\dot{\mathrm{Q}}_{\text {inf }}=$ Total load added to the case due to infiltration of room air, (Btu/hr)

$\dot{\mathrm{Q}}_{\mathrm{cr}}=$ Total refrigeration load of the case determined by refrigerant properties, $(\mathrm{Btu} / \mathrm{hr})$

This approach relies on a mass energy balance solution, which cannot be directly influenced by the airflow characteristics of the air curtain. Consequently, the effects of discharge air velocity, discharge grill dimensions, and other geometry related characteristics of the case did not play a direct role in determining the mass of warm and moist air entrained into the case. It is, however, more desirable to develop a methodology to quantify the infiltrated mass of air into the case based on the air curtain flow characteristics and case geometry. The flow rate of air into the display case was determined using Equation 60.

$$
\operatorname{cfm}_{\text {inf }}=\frac{\left(\mathrm{m}_{\mathrm{tc}}-\mathrm{m}_{\mathrm{cd}}\right)}{\left[\left(\omega_{\text {room }}-\omega_{\text {case }}\right) * \rho_{\text {ra }} * \mathrm{t}_{\mathrm{r}}\right]}
$$

$\mathrm{cfm}_{\mathrm{inf}}=$ Amount of entrained air from the room infiltrating into the display case, $\left(\mathrm{ft}^{3} / \mathrm{min}\right)$.

$\mathrm{m}_{\mathrm{tc}} \quad=$ Total mass of condensate collected over $24 \mathrm{hr}$ of test, (lb)

$\mathrm{m}_{\mathrm{cd}}=$ Total mass of water vapor condensed from air during defrost periods, (lb)

$\omega_{\text {room }}=$ Absolute humidity of air in the room, $\left(1 b_{\mathrm{w}} / \mathrm{lb}_{\mathrm{a}}\right)$

$\omega_{\text {case }}=$ Absolute humidity of air in the case, $\left(\mathrm{lb}_{\mathrm{w}} / \mathrm{lb}_{\mathrm{a}}\right)$

$\rho_{\mathrm{ra}}=$ Density of air in the room, $\left(\mathrm{lb} / \mathrm{ft}^{3}\right)$

$\mathrm{t}_{\mathrm{r}} \quad=$ Refrigeration period, (minutes)

Additionally, the sensible and latent load components of the total infiltration load were obtained. The sensible portion of the infiltration load was determined using Equation 61. 


$$
\begin{array}{ll}
\dot{\mathrm{Q}}_{\text {sense inf }} & =\rho_{\text {air }} * \operatorname{cfm}_{\text {inf }} * \mathrm{Cp}_{\text {air }} * \mathrm{k} *\left(\mathrm{~T}_{\text {room }}-\mathrm{T}_{\text {case }}\right) \\
\dot{\mathrm{Q}}_{\text {sense inf }} & =\text { Sensible part of the infiltration load, }(\mathrm{Btu} / \mathrm{hr}) \\
\rho_{\text {air }} & =\text { Density of air, } \mathrm{lb} / \mathrm{ft}^{3} \\
\mathrm{Cp}_{\text {air }} & =\text { Specific heat of air, }(\mathrm{Btu} / \mathrm{lb}) \\
\mathrm{k} & =\text { Conversion factor, }(60 \mathrm{~min} / \mathrm{hr})
\end{array}
$$

The latent portion of the infiltration was determined using Equation 62. In fact, the only source of latent load in this particular test was the ambient air, which entrained into the case across the air curtain. In some cases, additional moisture is generated within the display case as a result of product respiration, which can further increase the latent load of the fixture.

$\dot{\mathrm{Q}}_{\text {latentinf }}=\frac{\left[\left(\mathrm{m}_{\mathrm{tc}}-\mathrm{m}_{\mathrm{cd}}\right) * \mathrm{~h}_{\mathrm{fg}}\right]}{\mathrm{t}_{\mathrm{r}}}$

$\dot{\mathrm{Q}}_{\text {latent inf }}=$ Latent part of the infiltration load, (Btu/hr)

$\mathrm{h}_{\mathrm{fg}}=$ Heat of vaporization of water (based on average evaporator coil surface temperature), (Btu/lb) 


\section{TEST RESULTS}

\subsection{Baseline Display Case}

The following describes the specifications for the four-deck open vertical deli/meat merchandiser that was tested to establish the baseline scenario. Figures 51 and 52 depict a photograph and the schematic diagram of the case with all the important dimensions.

$\begin{array}{ll}\text { Evaporator: } & 81 \text { in. W x } 7-5 / 8 \text { in. } \mathrm{H} \times 12 \text { in. D } \\ & 4 \text { refrigerant circuits } \\ & 12 \text { tube passes per circuit, total of } 48 \\ & \text { copper tube thickness of } 0.016, \text { in. } \\ & \text { tube outside diameter of } 9 / 16 \text { in. } \\ & \text { tube inside diameter of } 0.5305, \text { in. } \\ & 171 \text { aluminum plain type fins } \\ & \text { fin thickness of } 0.008 \text { in. } \\ & 2 \text { fins per inch } \\ & \text { Single band } \\ \text { Air Curtain: } & \text { Three } 9 \mathrm{~W} \text { (output) standard fans, } \\ \text { Evaporator Fan Motor: } & \text { (Model No. 5KSM5ECG 5132) } \\ & 8 \text { in., } 5 \text { blade, } 31 \text { deg pitch } \\ \text { Evaporator Fan Blade: } & 1 \text { in. x } 4 \text { in. x } 48 \text { in. grid } \\ \text { Honeycomb: } & \text { Four } \\ \text { Number of Shelves: } & \text { Thermostatic Expansion (Sporlan SBFRE A C) } \\ \text { Expansion Valve: } & \text { Off-cycle } \\ \text { Defrost Type: } & \text { Three per day } \\ \text { Defrost Frequency: } & 45 \text { min (fail-safe) } \\ \text { Defrost Length: } & 47^{\circ} \mathrm{F}\end{array}$

\section{Refrigeration Data}

Refrigerant:

Discharge air:

Discharge air velocity:

Return air:

Evaporator:

Conventional capacity:

Superheat Set point:
$\mathrm{R}-404 \mathrm{~A}$

$28^{\circ} \mathrm{F}$

$350 \mathrm{fpm}$

$37^{\circ} \mathrm{F}$

$17^{\circ} \mathrm{F}$

$1,561 \mathrm{BTUH}$ at $17^{\circ} \mathrm{F}$

6 to $8^{\circ} \mathrm{F}$ 


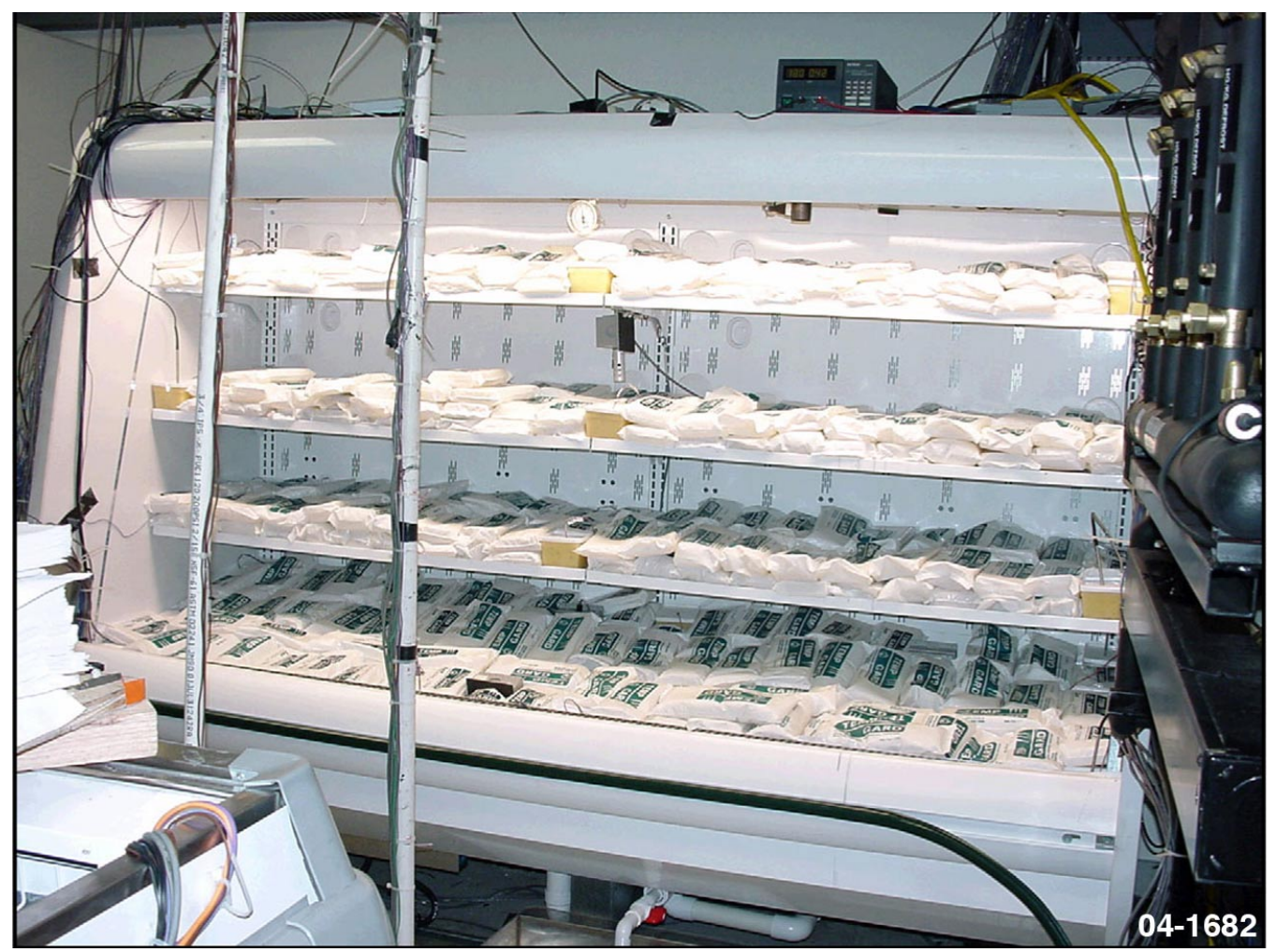

Figure 51. Photograph of the four-deck deli display case

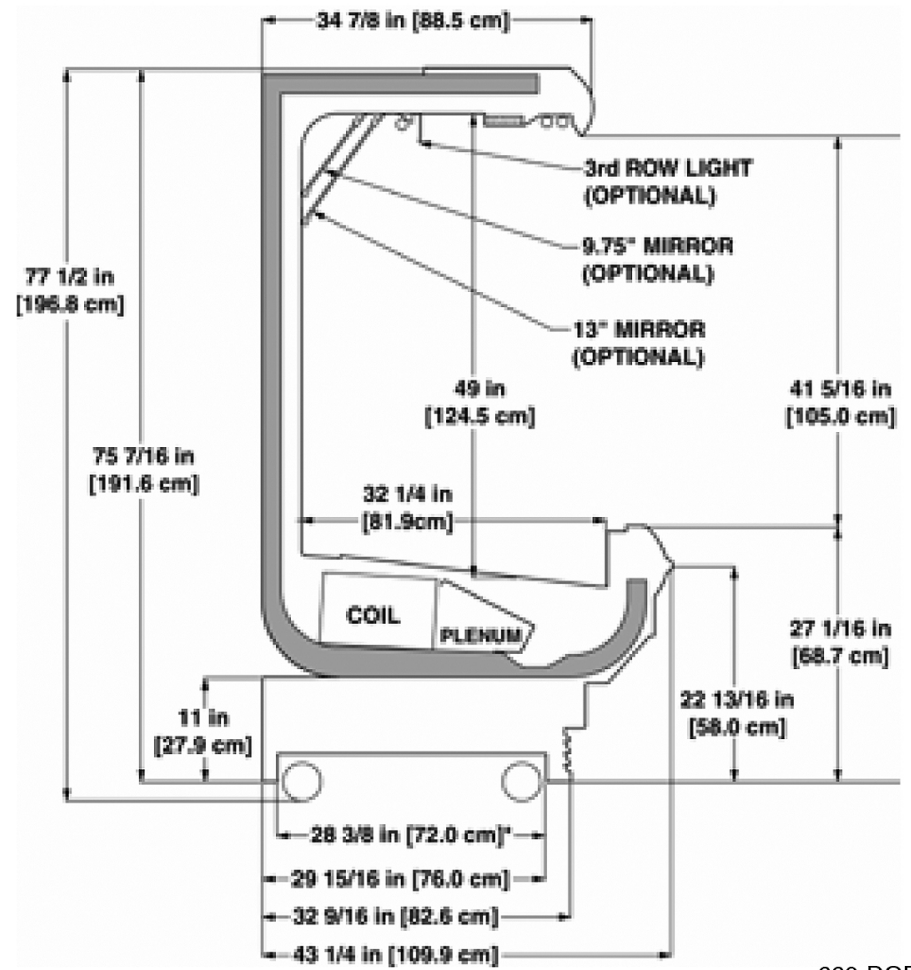

660-DORL-010231-42

Figure 52. Schematic of the four-deck deli display case (courtesy of Hill Phoenix) 


\section{Electrical and Physical Data}

Fans:

Standard Lighting:

$1.50 \mathrm{~A}$

Anti-Condensate Heaters:

$0.57 \mathrm{~A}$ per light row

Fan and Lights Voltage:

$0.58 \mathrm{~A}$

Anti-Condensate Voltage:

$120 \mathrm{~V}$

Drip Pipe Size:

$120 \mathrm{~V}$

2 in. PVC

\subsection{Baseline Test Results}

The performance of the baseline display case was evaluated using ASHRAE 72-98 test conditions. The test was run for a period of $24 \mathrm{hr}$, however, prior to initiating the test run, the controlled environment room was allowed to reach a steady-state equilibrium condition.

Figure 53 illustrates the 2 min profile of the controlled environment room DB and RH during the entire test period. As illustrated, the indoor conditions remained fairly unchanged during the entire test period. The average room DB and RH was $75.1^{\circ} \mathrm{F}$ and 54.5 percent, respectively, which corresponded to a wet-bulb (WB) of $63.7^{\circ} \mathrm{F}$.

The test rack controller was programmed to run at a fixed suction pressure of $52 \mathrm{psig}$, which corresponded to the evaporator temperature of $17^{\circ} \mathrm{F}$ defined by the manufacturer. The rack controller was also programmed to run at a fixed discharge pressure of 220 psig or $95^{\circ} \mathrm{F} \mathrm{SCT}$. Figure 54 illustrates the 2 min profile of suction and discharge pressures over the entire test period.

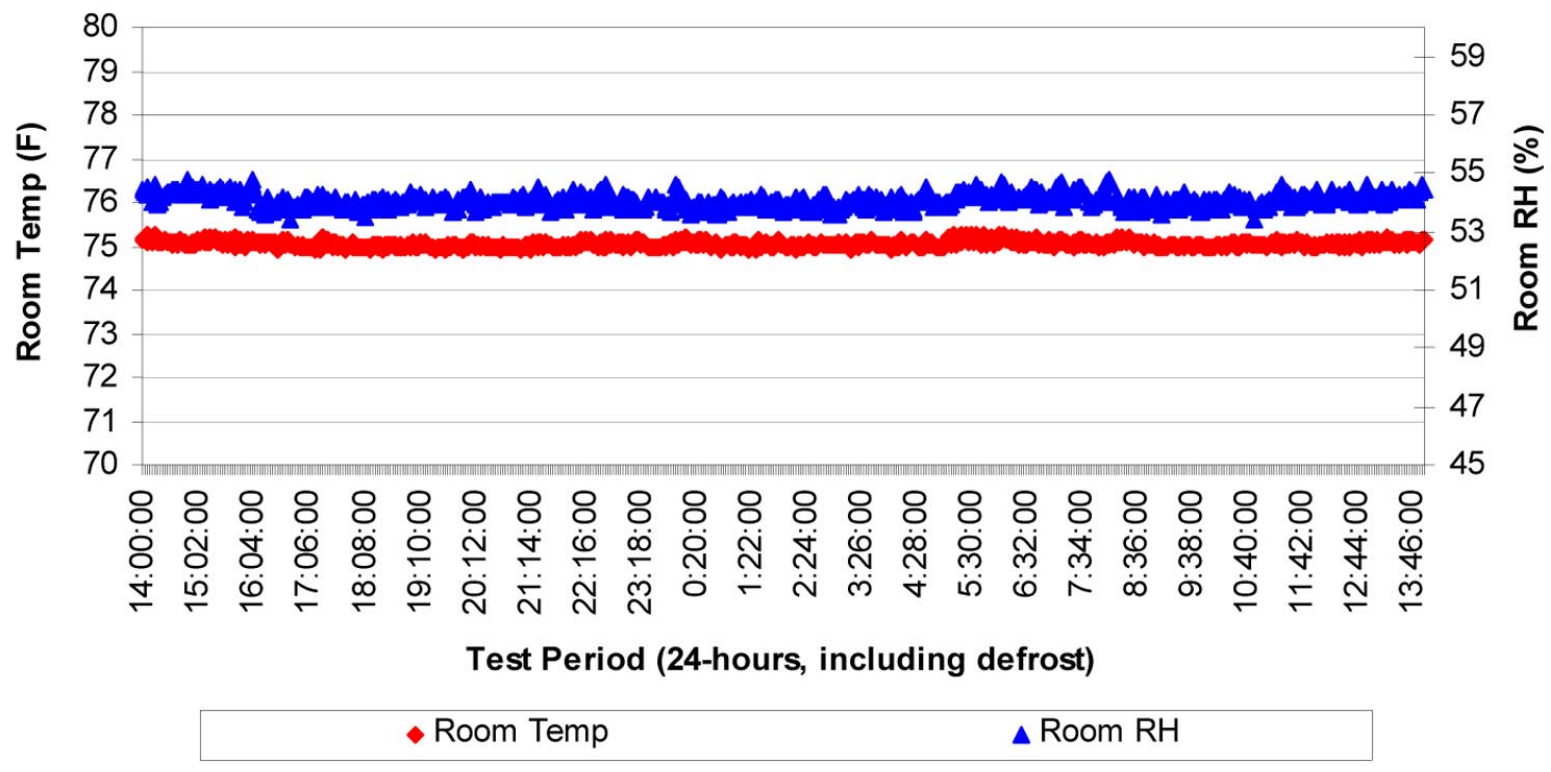

660-DORL-010231-43

Figure 53. 2 min profile of the controlled environment room DB and RH over $24 \mathrm{hr}$ 


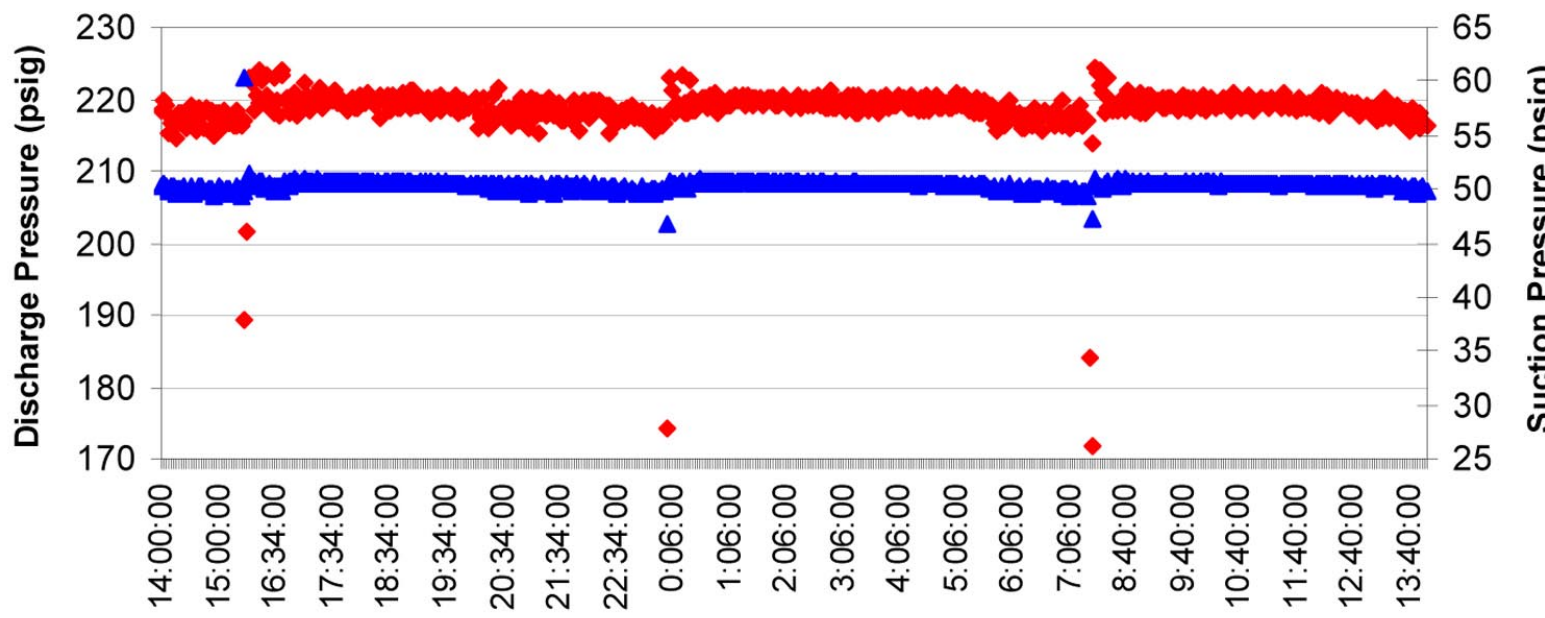

Test Period (24-hours, excluding defrost)

$\bullet$ Discharge Pressure $\Delta$ Suction Pressure

660-DORL-010231-44

\section{Figure 54. 2 min profile of suction and discharge pressures over $24 \mathrm{hr}$}

Figure 55 depicts the 2 min profile of condensate mass collected over the entire test period. This condensate mass comprised of moisture collected from the moist air stream during refrigeration run time and melted ice (or frost) during off-cycle defrost periods. The stepped (horizontal) profiles indicate the collected moisture during refrigeration run time between each of three defrost periods. The vertical (or sloped) profiles, on the other hand, indicate the melted ice during each defrost cycle.

In addition to condensate collection during refrigeration and defrosts' ice melting, further moisture was detected to escape from the air during defrost. During off-cycle defrost periods, compressor stops running while the evaporator fan motors continue operation, thereby bringing relative warm and humid air into the case. As a result, the room's warm and moist air was the main factor responsible for melting the ice on the coil. Figure 56 shows the subcomponents of condensation. Clearly, the most condensate removal took place during the ice melting stages of defrost cycle $(76.4 \mathrm{lb})$.

Bringing warm and humid indoor air into the case to melt frost on the coil caused the temperature and RH inside the fixture to increase and reach maximum levels during defrost periods (Figure 57). Once the refrigeration period was initiated, it lowered the temperature and humidity inside the case. However, as the refrigeration period continued, the temperature and humidity levels started to increase until the next defrost period was reached. This observation can be attributed to decrease in evaporator coil capacity due to increase in frost formation on the coil. 


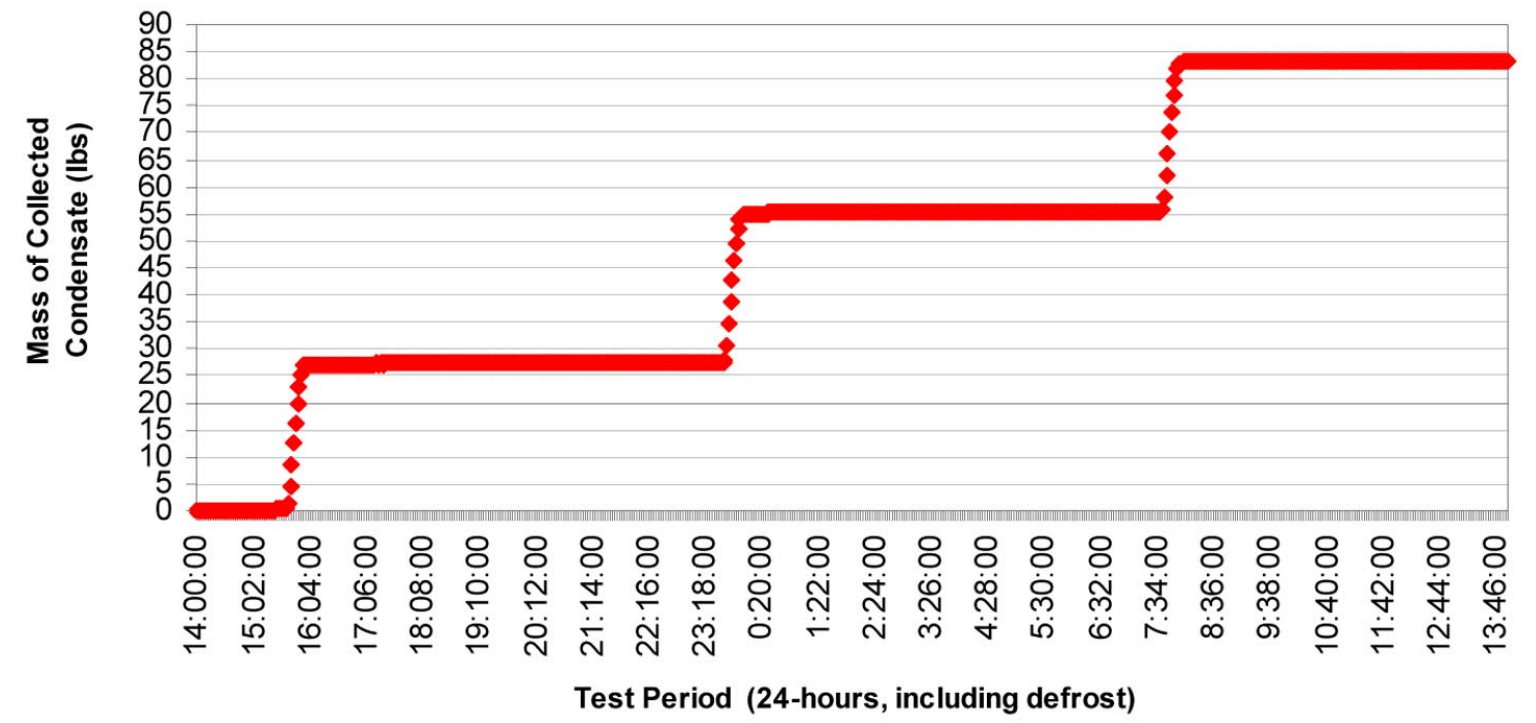

660-DORL-010231-48

Figure 55. 2 min profile of collected condensate over $24 \mathrm{hr}$

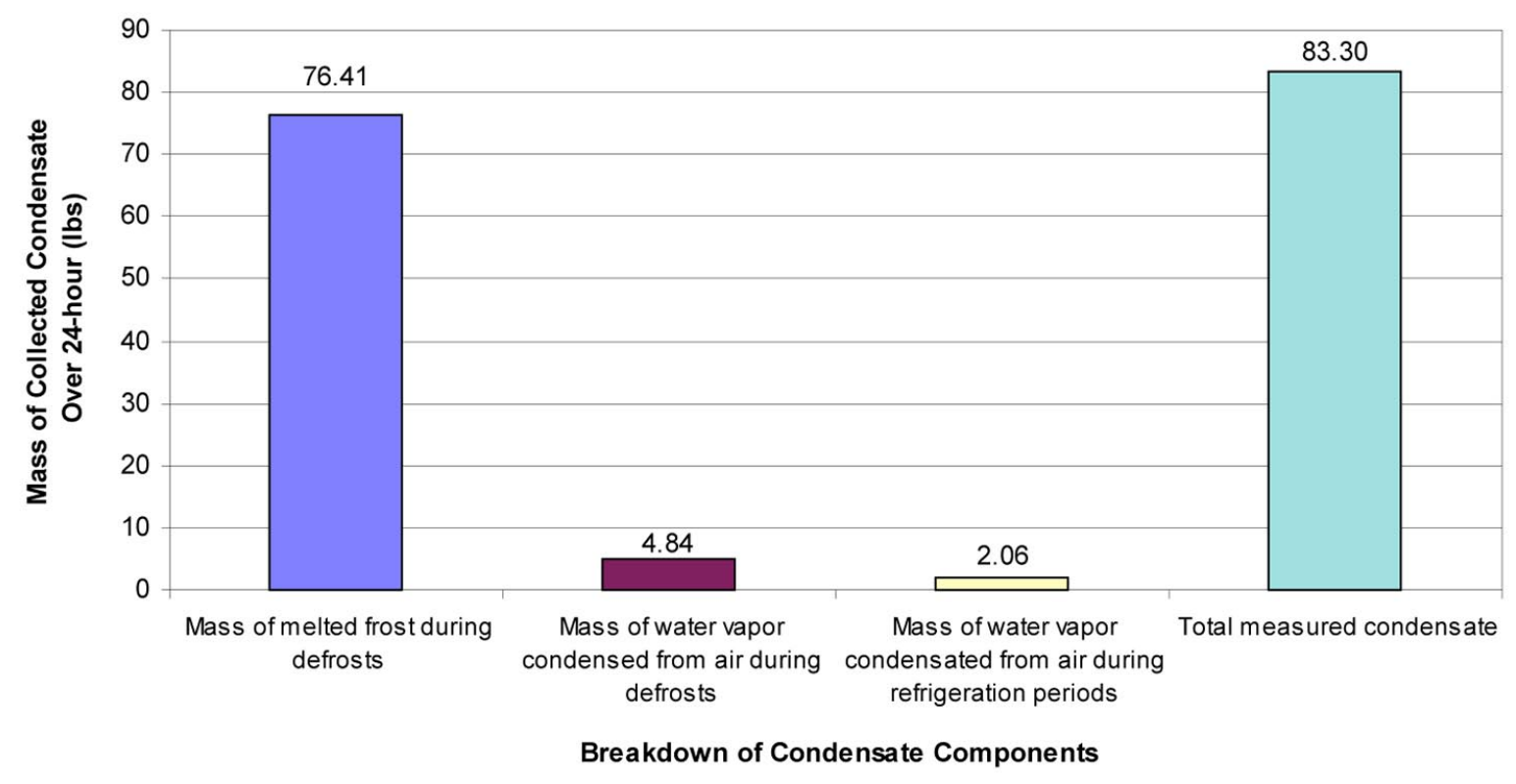

660-DORL-010231-45

Figure 56. Breakdown of collected condensate over $24 \mathrm{hr}$ 


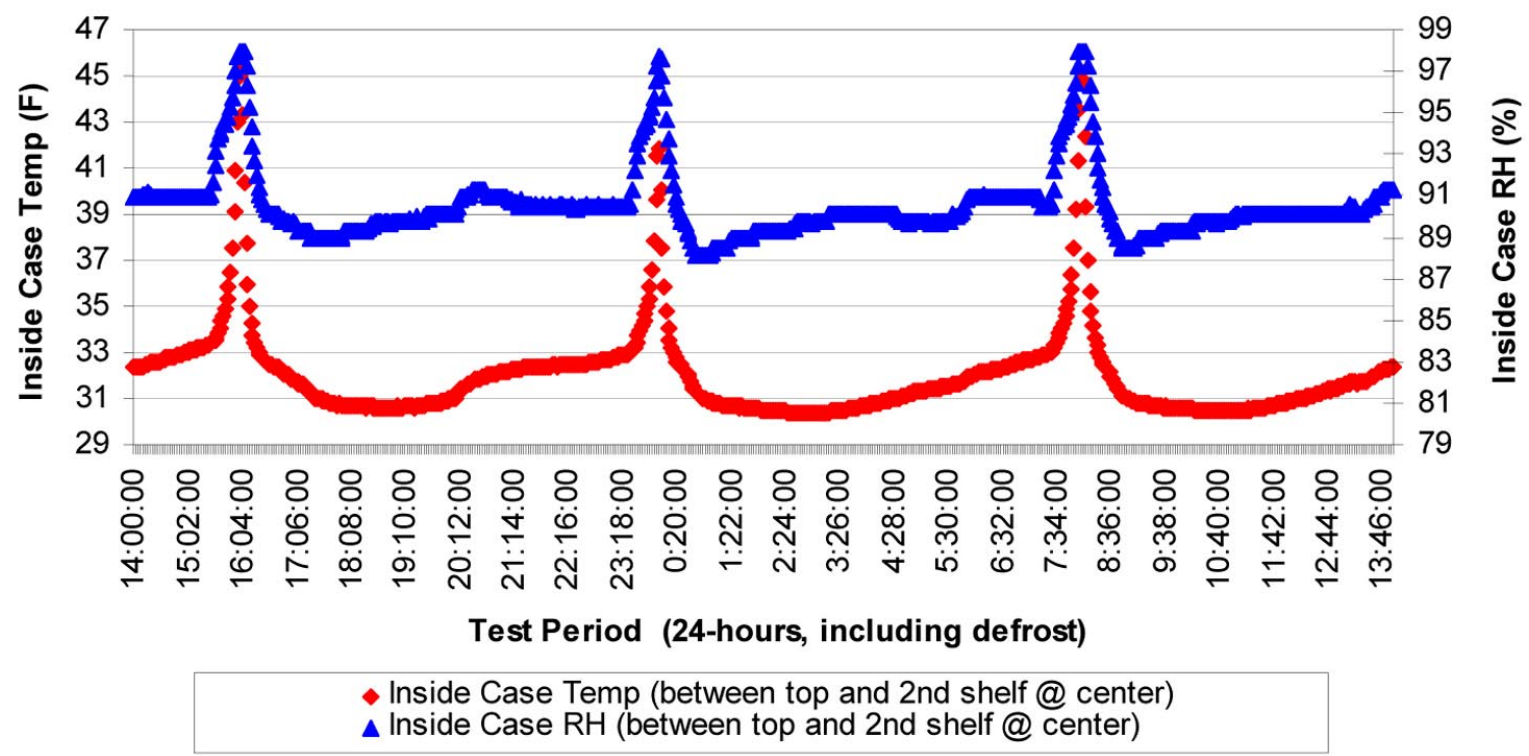

660-DORL-010231-46

Figure 57. 2 min profile of display case's temperature and $\mathrm{RH}$ over $24 \mathrm{hr}$

The increase in frost layers caused resistance or blockage to the passage of the air being circulated by the evaporator fans inside the case. This resistance or blockage is evident as an increase in static pressure differential $(\Delta \mathrm{P})$ across the evaporator coil (Figure 58$)$. There is no change in air pressure during and roughly $1 \mathrm{hr}$ after defrost period. However, as the refrigeration continues and frost layers start building up on the coil, the $\Delta \mathrm{P}$ increases accordingly.

The static pressure drop across the coil during refrigeration periods, due to increase in mass of frost, resulted in a downward sloped profile in discharge air velocity (DAV), as shown in Figure 59. The average DAV reached its maximum limit at the end of defrosts, and its minimum limit prior to initiation of defrosts.

Further, the magnitude of DAV varied along the honeycomb or DAG while the velocity profiles remained identical for all four locations. Figure 60 illustrates that the air circulation inside the case was non-uniform and it varied depending on the location. The highest velocity magnitude was observed to be at the center of DAG, and the lowest magnitude at the right center location.

Figure 61 depicts the total cooling load per linear feet of the case. The highest cooling load was observed at the end of each defrost period due to bringing relatively warm and humid air into the case during defrosts. The lowest cooling load was observed prior to initiation of defrosts.

The variations in sensible load coupled with a relatively constant latent load profile explained 


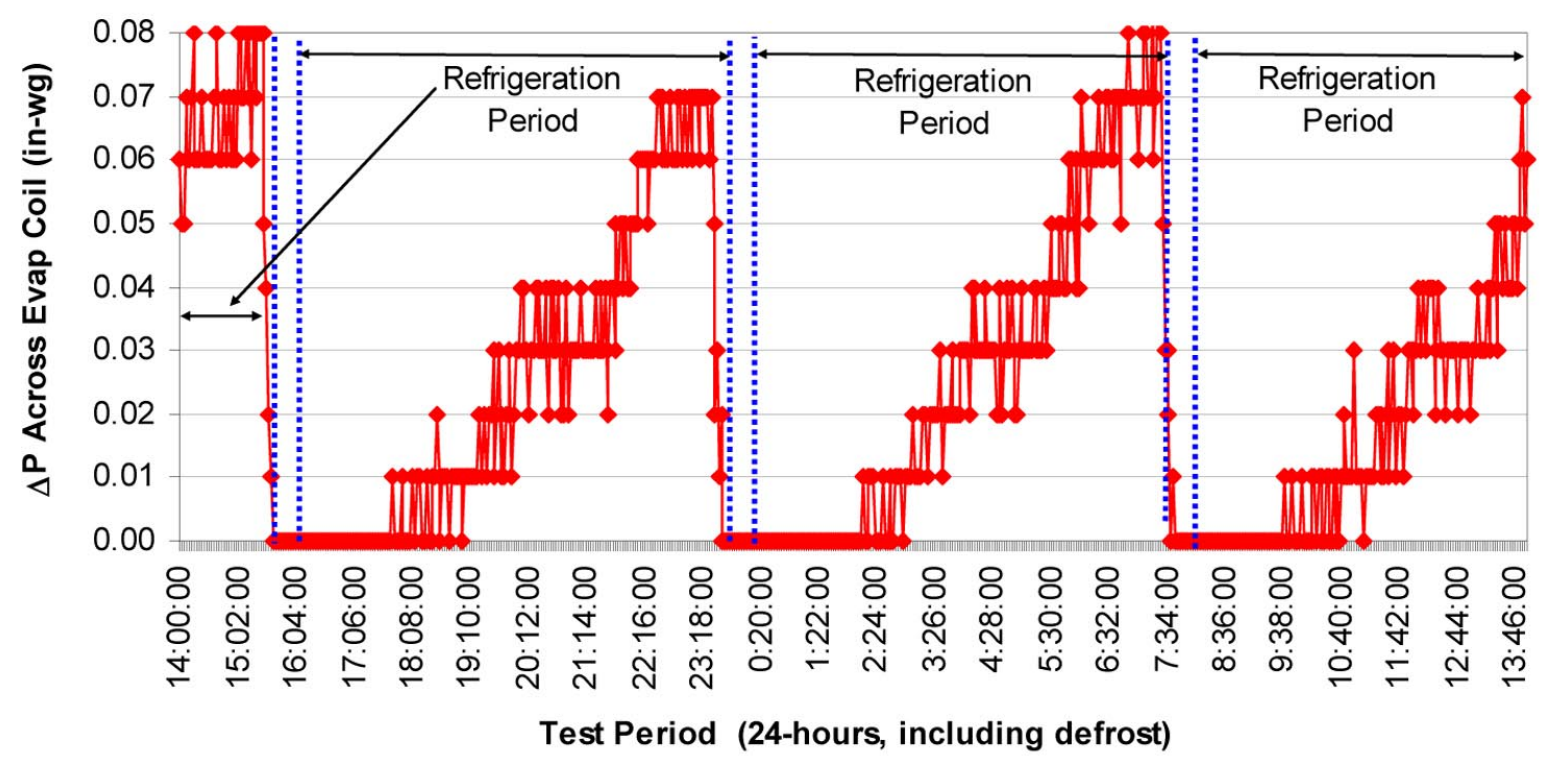

660-DORL-010231-47

Figure 58. 2 min profile of pressure differential across the evaporator coil over $24 \mathrm{hr}$

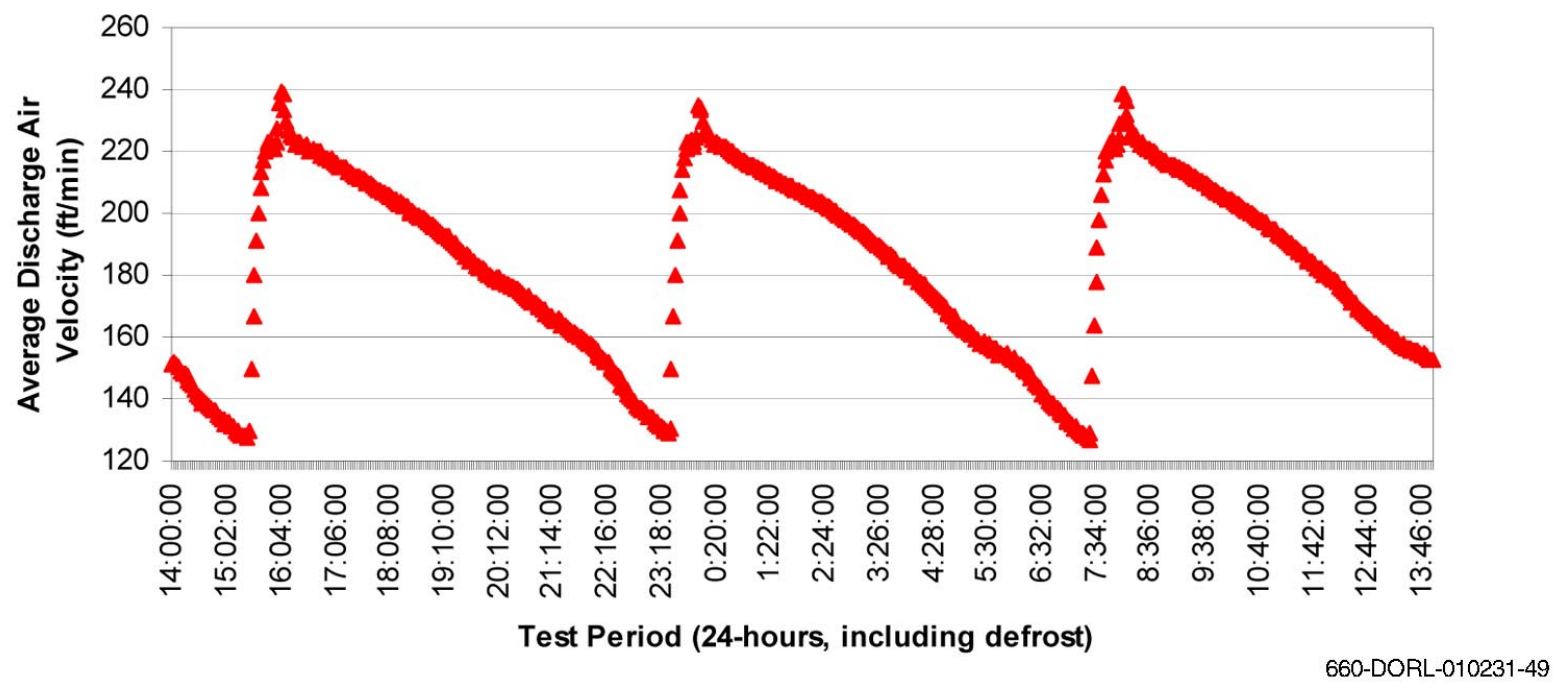

Figure 59. 2 min profile of average DAV over $24 \mathrm{hr}$ 


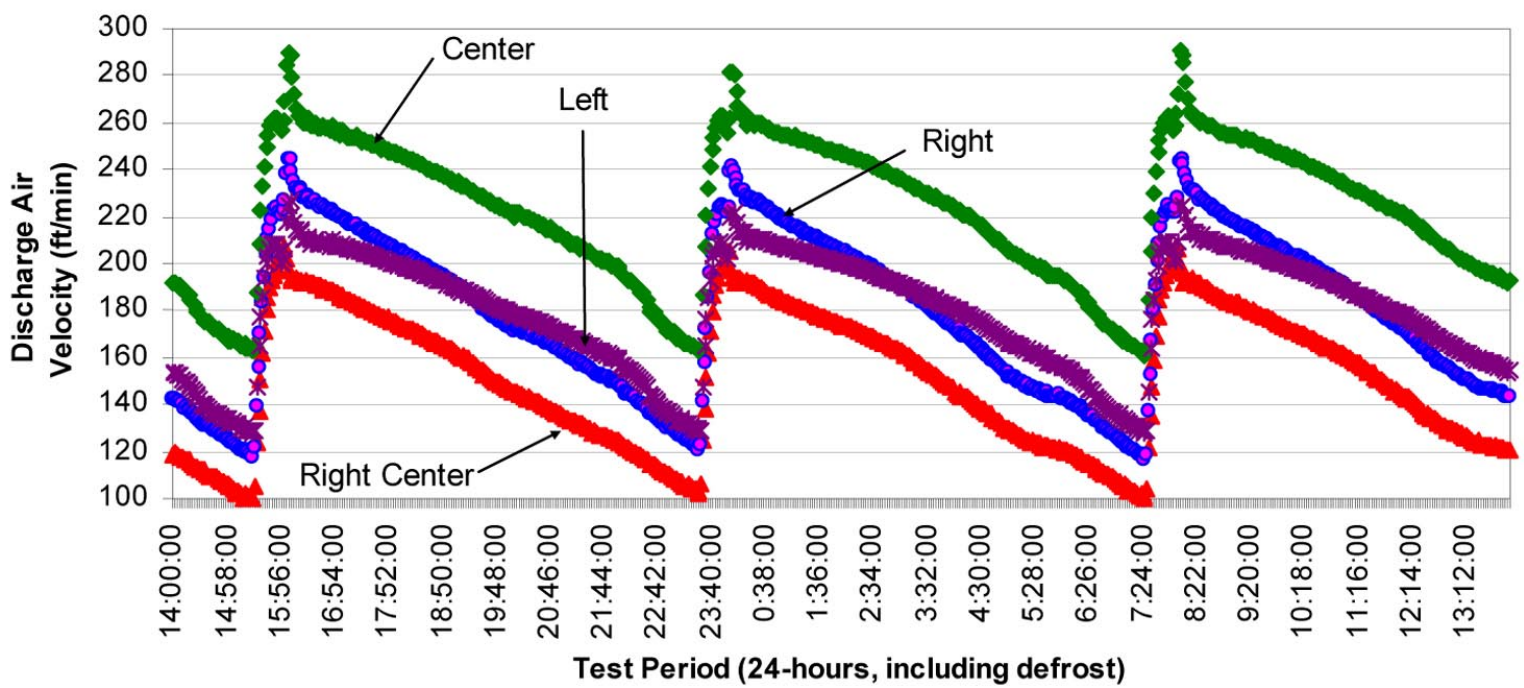

$\Delta$ Right Center - Right - Center * Left

660-IBDP-010231-50

Figure 60. 2 min profile of DAV along the honeycomb (or DAG) over $24 \mathrm{hr}$

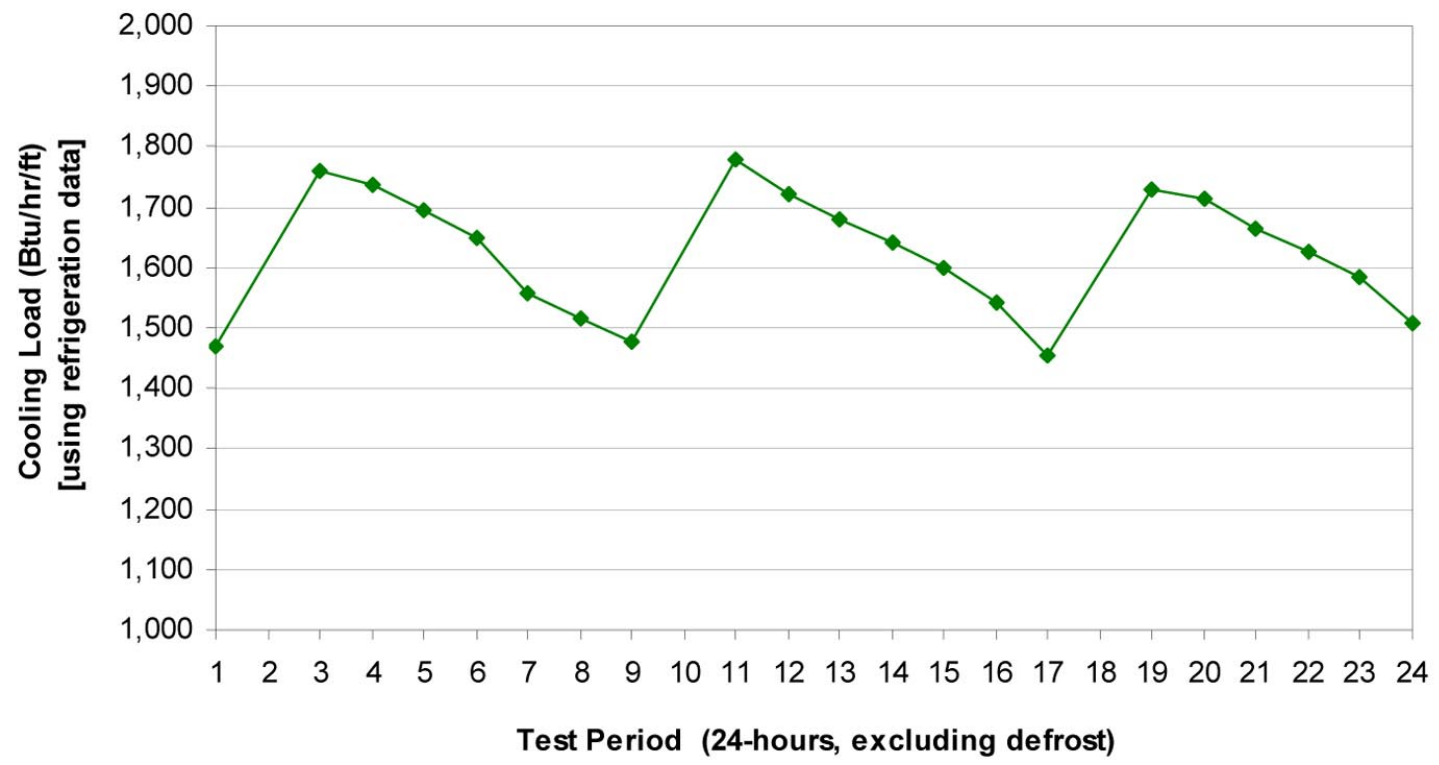

660-DORL-010231-51

Figure 61. Hourly profile of total cooling load per linear feet of the fixture over $24 \mathrm{hr}$ 
the variations in total cooling load of the case (Figure 62). The latent load of the fixture remained fairly constant during the entire test period, and it relied on the actual mass of water measured at the end of the test. The sensible load of the case increased at the end of defrosts and it decreased prior to initiation of defrosts. This observation can be attributed to increase in the display case's temperature during defrost periods.

Figure 63 depicts the total cooling load and its components (latent and sensible) using psychrometric (air-side) and refrigeration data. The difference between the two lies in the different methodologies used to determine the weight of condensate. The refrigeration approach relied on the actual mass of water measured at the end of the test while the other estimated the mass of water removed based on the air flows as well as temperature and RH of the air before and after the coil. Both these approaches yielded relatively similar cooling load magnitudes.

As illustrated in Figures 64 and 65, the total cooling load of the fixture was constituted of infiltration, radiation, conduction, and internal loads (lights and evaporator fans). The largest component of the cooling load was infiltration with $10,674 \mathrm{Btu} / \mathrm{hr}$, which corresponded to 82 percent of total cooling load. The smallest component, on the other hand, was conduction with $526 \mathrm{Btu} / \mathrm{hr}$, and it contributed to 4 percent of the total cooling load. The display case's lighting system and evaporator fan motors contributed to 6 percent of the total cooling load. The radiation accounted for 8 percent of the total cooling load.

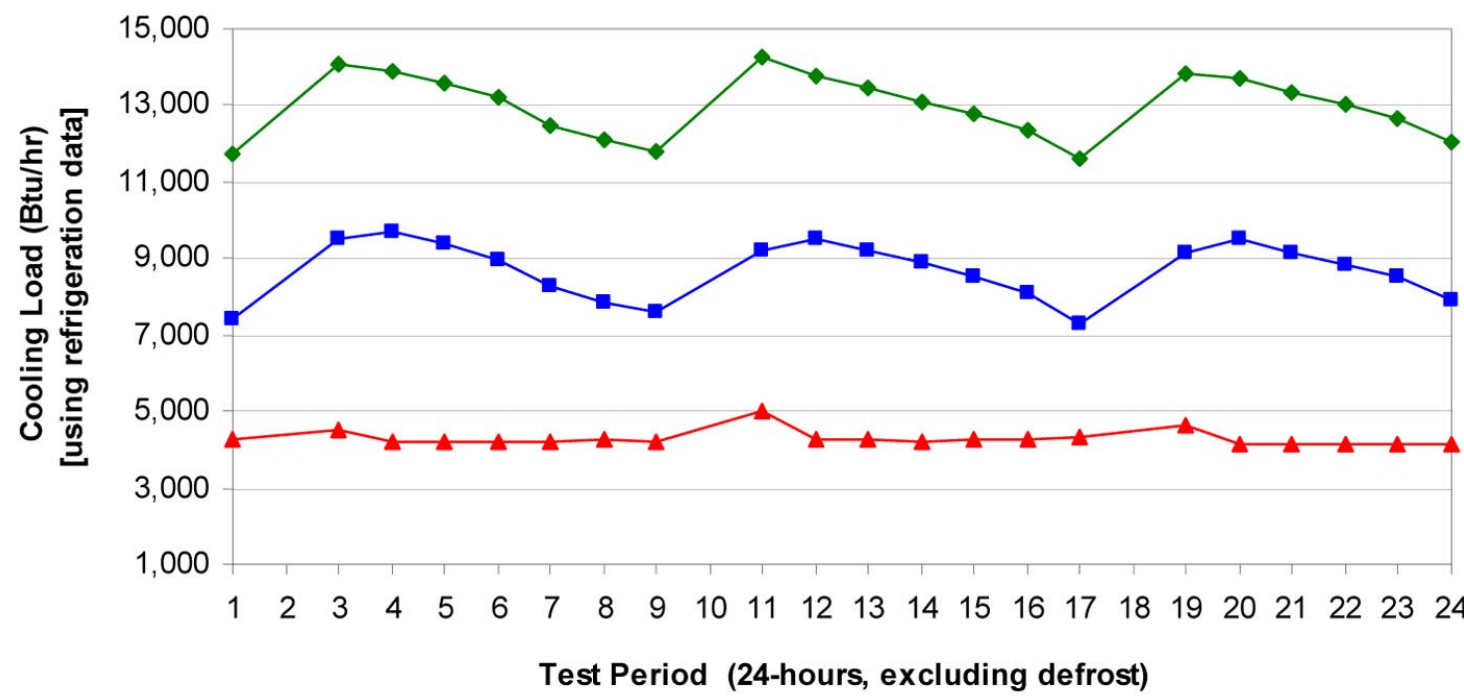

$\rightarrow-$ Total Cooling Load $\rightarrow-$ Sensible Cooling Load $\rightarrow$ Latent Cooling Load

660-DORL-010231-52

Figure 62. Hourly profile of total, sensible and latent cooling load over $24 \mathrm{hr}$ 


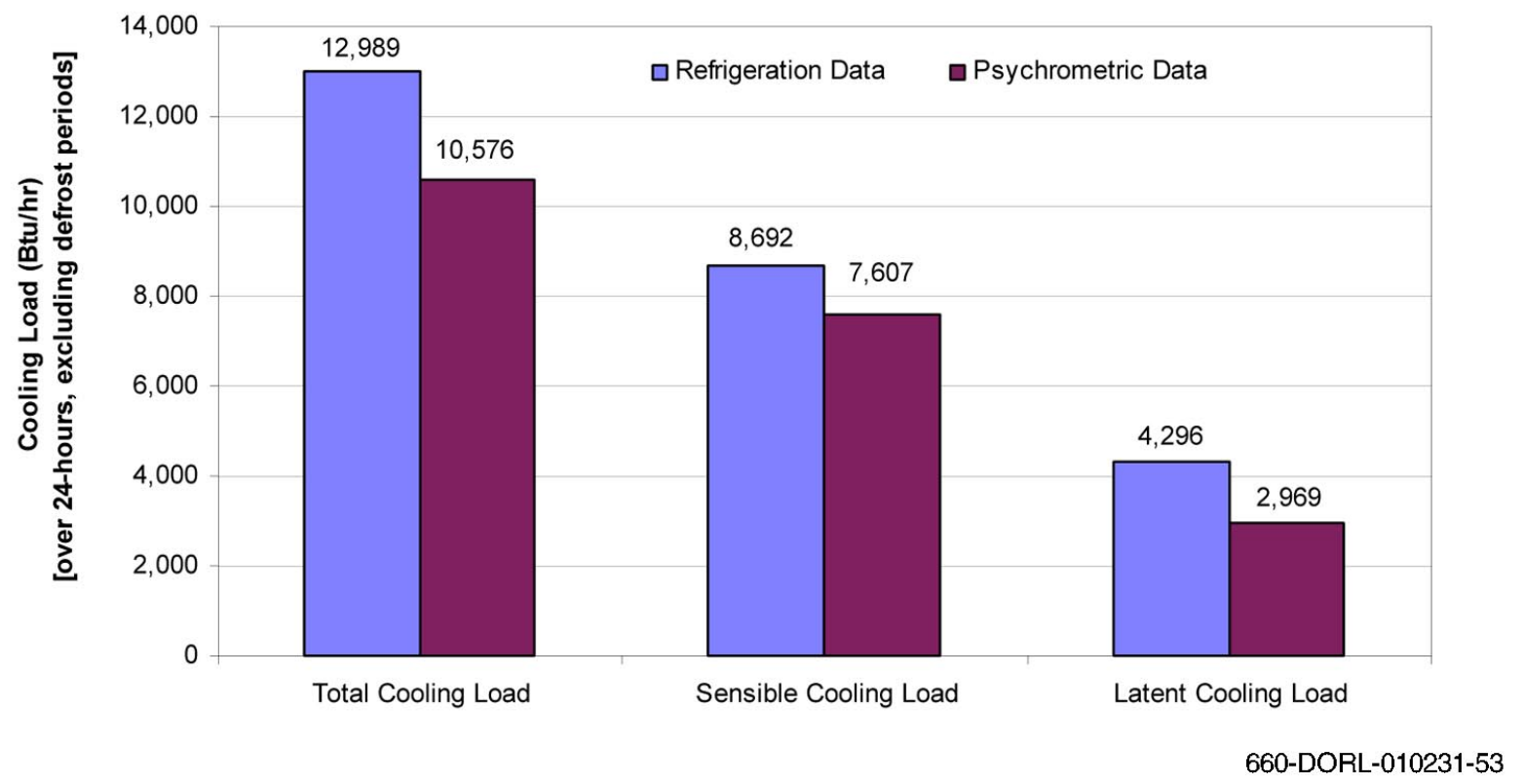

Figure 63. Total cooling load and its components using psychrometric and refrigeration data over $24 \mathrm{hr}$

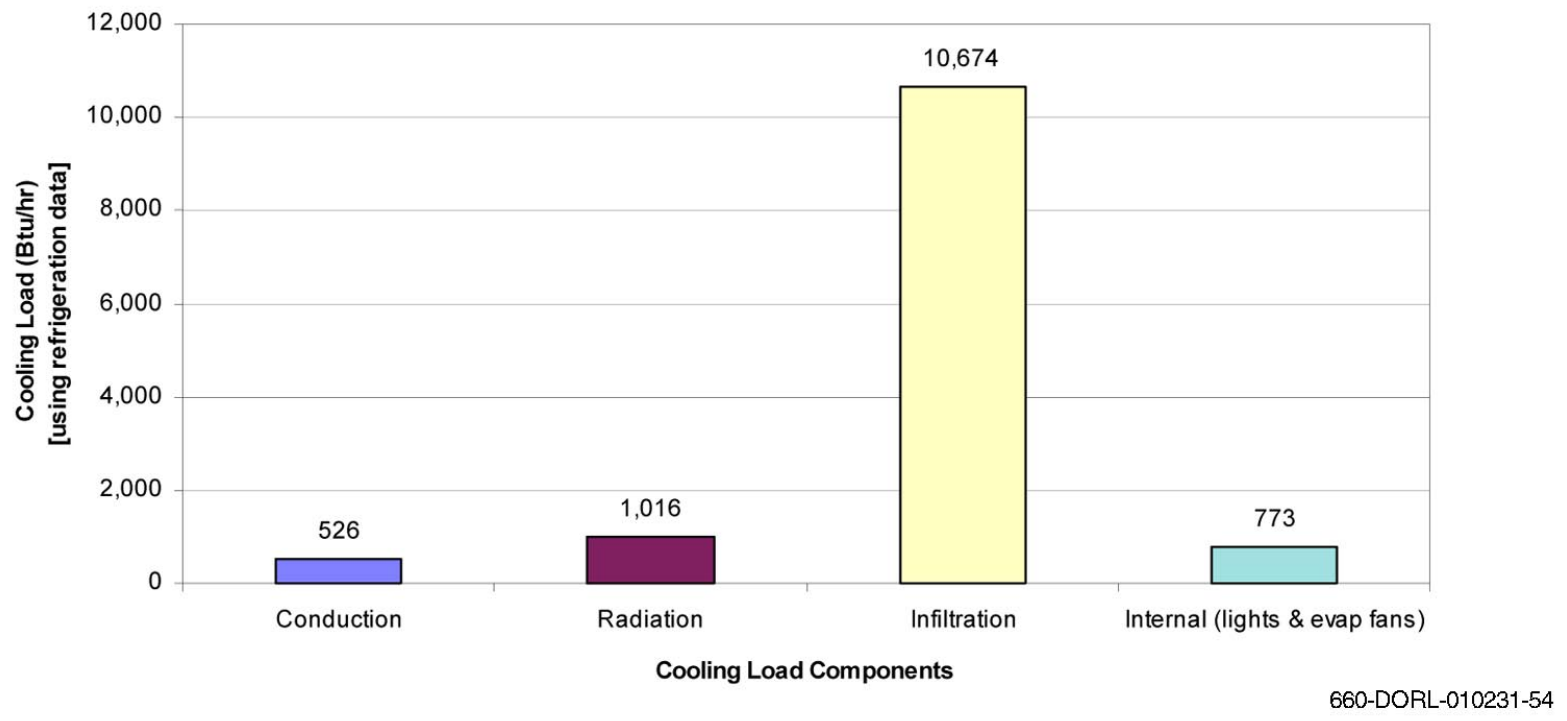

Figure 64. Cooling load components over $24 \mathrm{hr}$ 


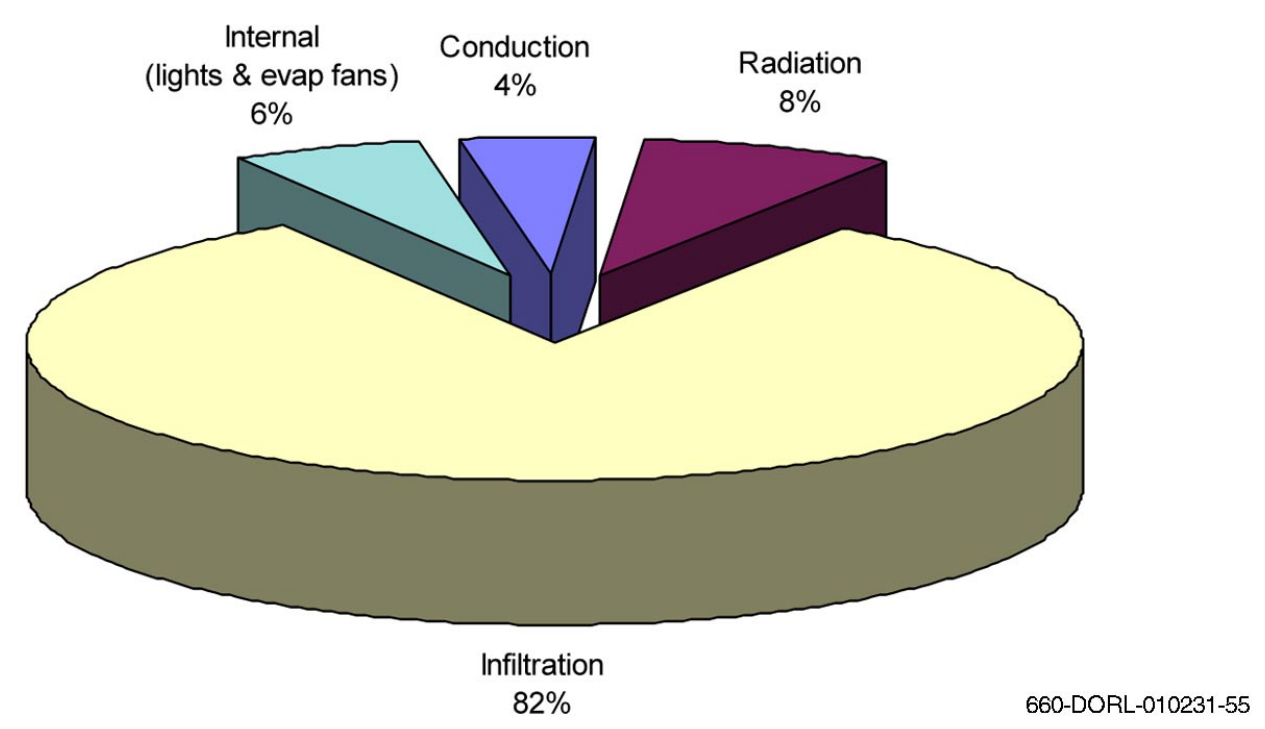

Figure 65. Percentage breakdown of the cooling load components over $24 \mathrm{hr}$

Additionally, the reduced cooling load, and average cooling load over the entire test period and during the last $3 / 4$ of the running cycle was determined according to ASHRAE Standard 7298 (Figure 66). Running cycle refers to the refrigeration period between two defrost periods. The methodology for determining these cooling loads was discussed in Section 9.

A descending trend in mass flow rate of refrigerant between each of three defrost cycles was observed (Figure 67). The mass flow rate of refrigerant was highest at the end of defrost and lowest prior to initiation of defrost. This observed profile in refrigerant mass flow rate could be attributed to change in total cooling load of the case coupled with maintaining a fixed suction pressure during the entire test period.

Comparing the actual 2 min compressor power and refrigerant mass flow rate profiles revealed a close similarity in behavior between the two parameters (Figure 68), as expected. That is, maintaining a fixed suction and discharge pressures caused the compressor power use to be entirely dependent on refrigerant mass flow rate variations.

Further comparison was made between refrigerant mass flow rate, and discharge air temperature (DAT) and velocity (Figure 69). This comparison revealed that DAT reached its lowest temperature at the end of defrosts due to frost or ice removal from the coils, which increased DAV, coupled with an increase in refrigerant mass flow rate. Once the refrigerant mass flow rate and DAV started to decline, which was an indication of increase in frost buildup on the coils, the DAT started to increase.

Figure 70 depicts the hourly profile of temperature differential (TD) between the saturated evaporating temperature and DAT. As depicted, the coil TD was highest at the end of defrosts and it started to decline as the refrigeration period begins. The average coil TD over $24 \mathrm{hr}$ of test run remained around $10^{\circ} \mathrm{F}$. The observed coil TD profile could be attributed to maintaining a 


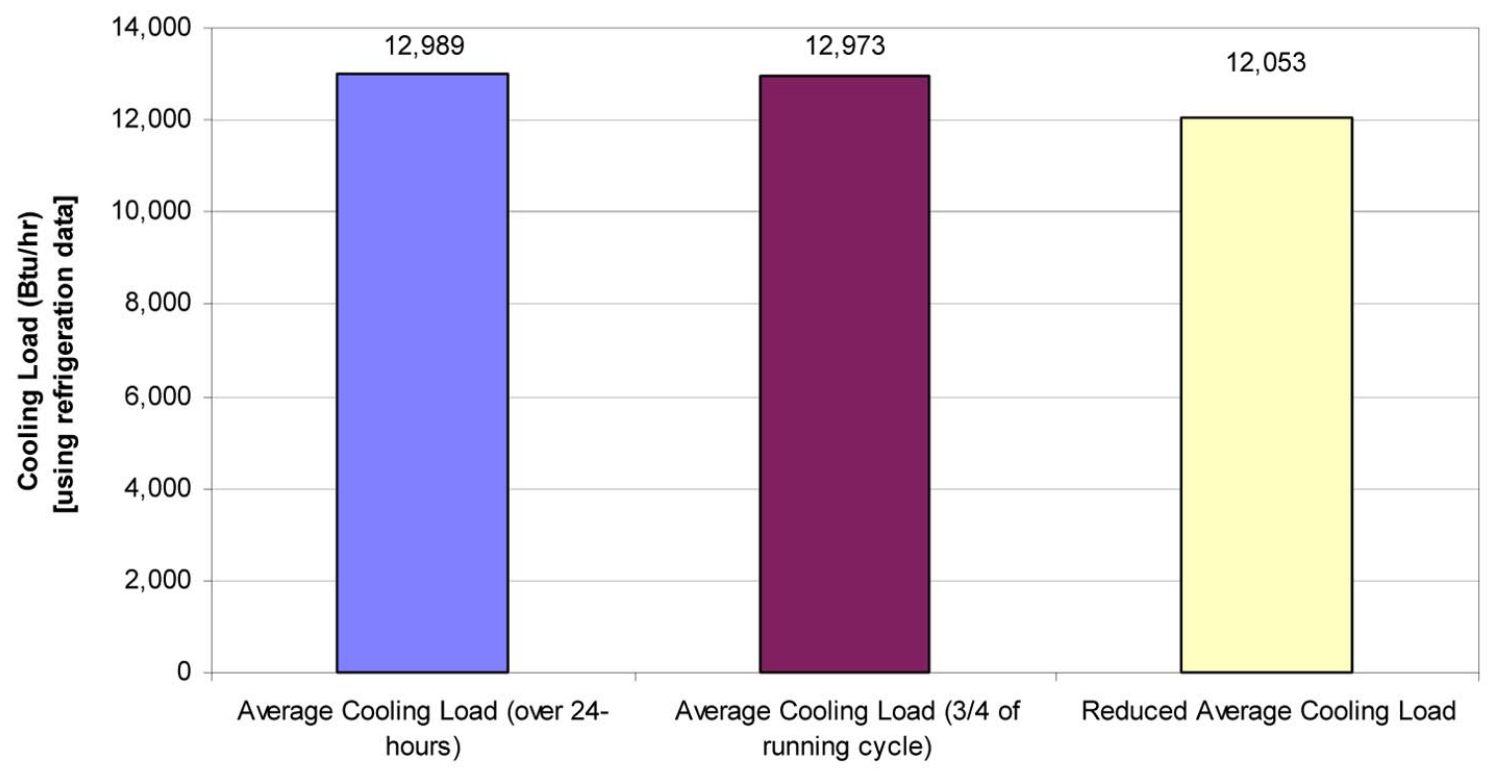

660-DORL-010231-56

Figure 66. Reduced cooling load, and average cooling load over $24 \mathrm{hr}$ and $3 / 4$ of running cycle

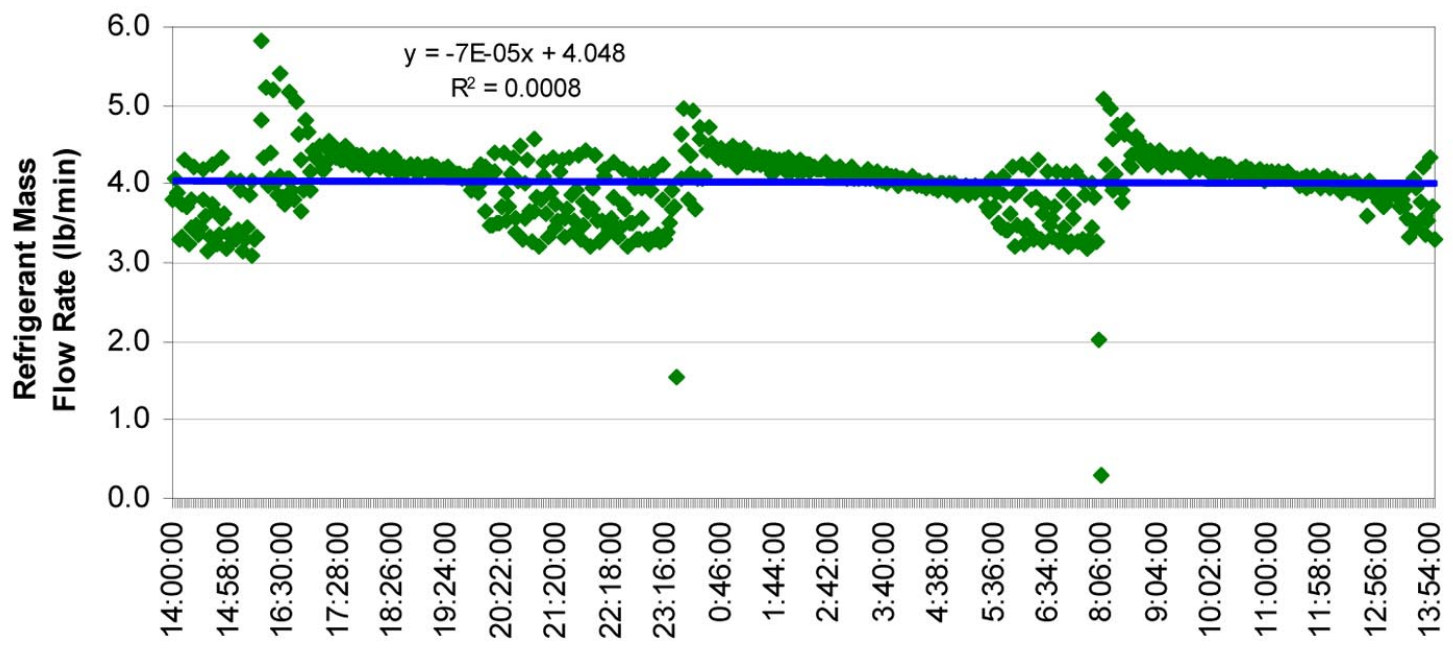

Test Period (24-hours, excluding defrost)

Figure 67. 2 min profile of refrigerant mass flow rate over $24 \mathrm{hr}$ 


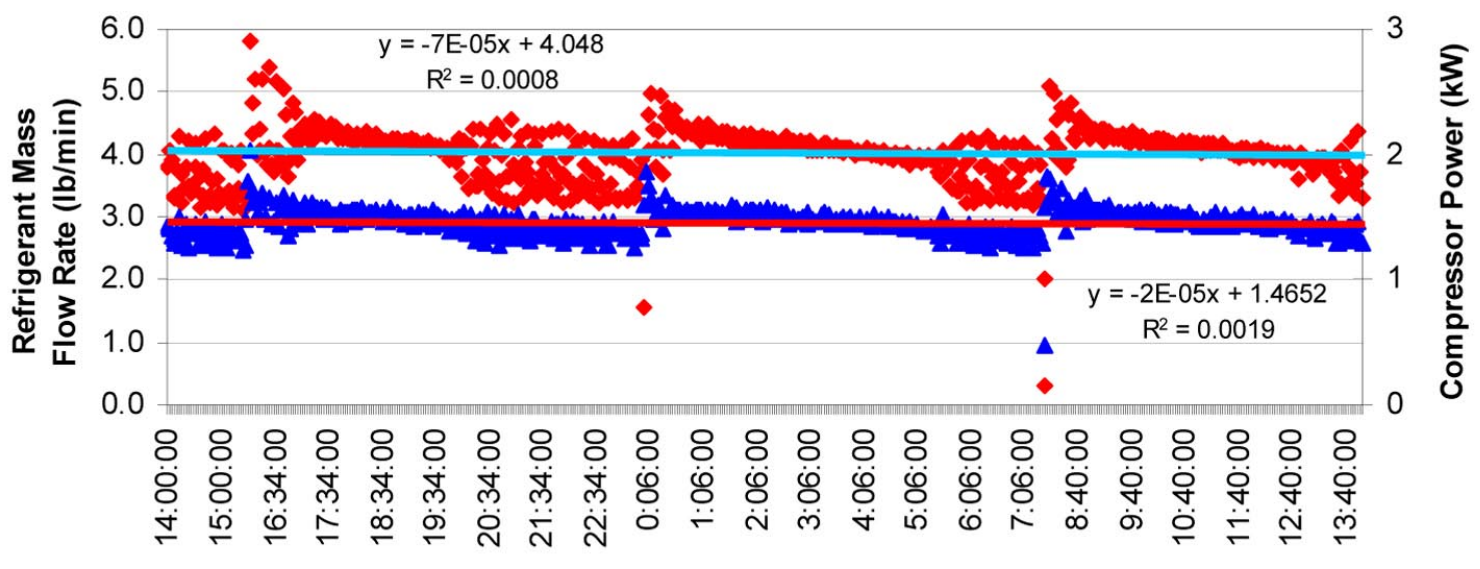

Test Period (24-hours, excluding defrost)

$\begin{array}{ll}\text { Refrigerant Mass Flow Rate } & \Delta \quad \text { Actual Compressor Power } \\ & \text { Linear (Refrigerant Mass Flow Rate) } \quad \text { Linear (Actual Compressor Power) }\end{array}$

660-DORL-010231-58

Figure 68. 2 min profiles of actual compressor power and refrigerant mass flow rate over $24 \mathrm{hr}$

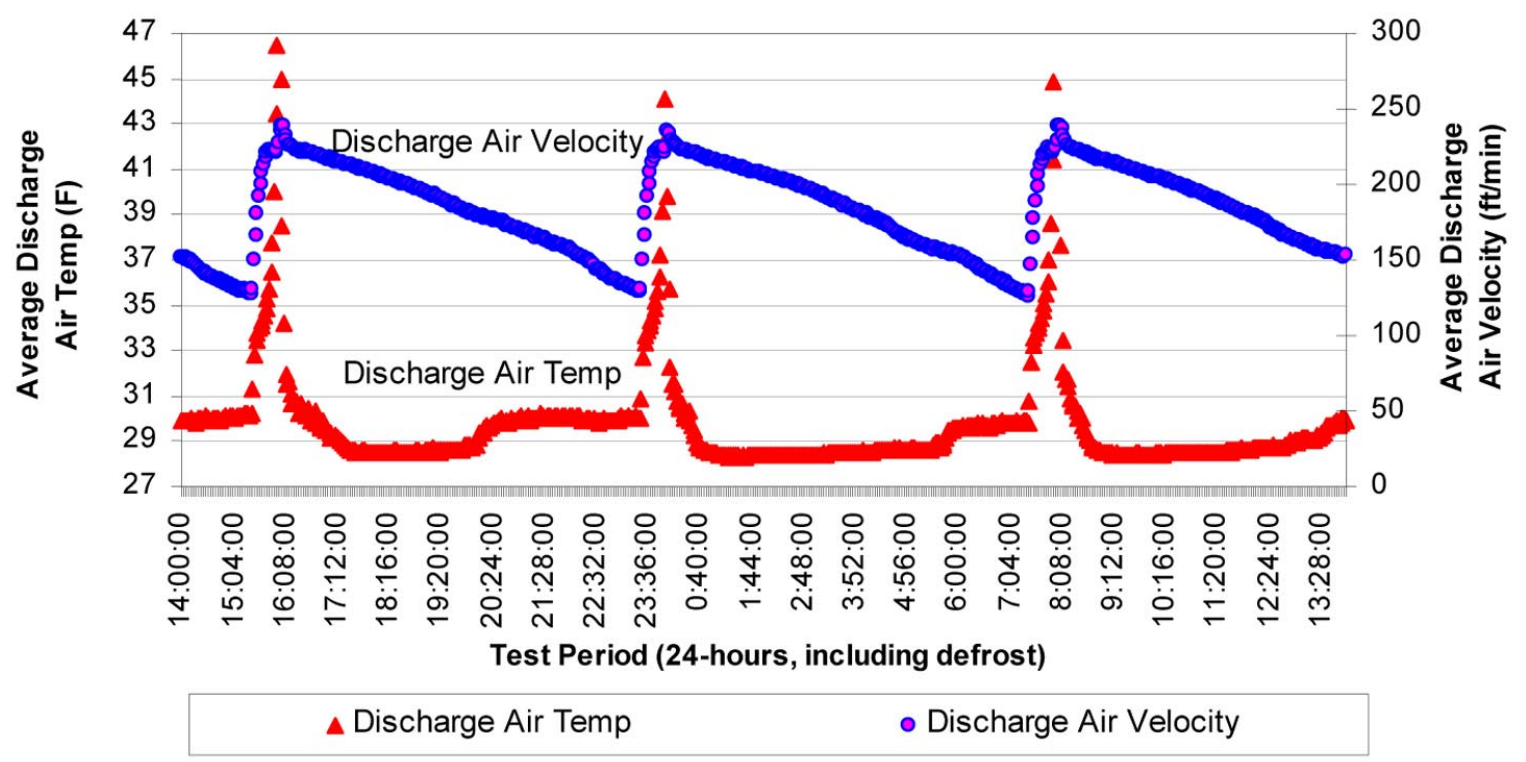

660-DORL-010231-59

Figure 69. 2 min profile of average discharge air velocity and temperature over $24 \mathrm{hr}$ 


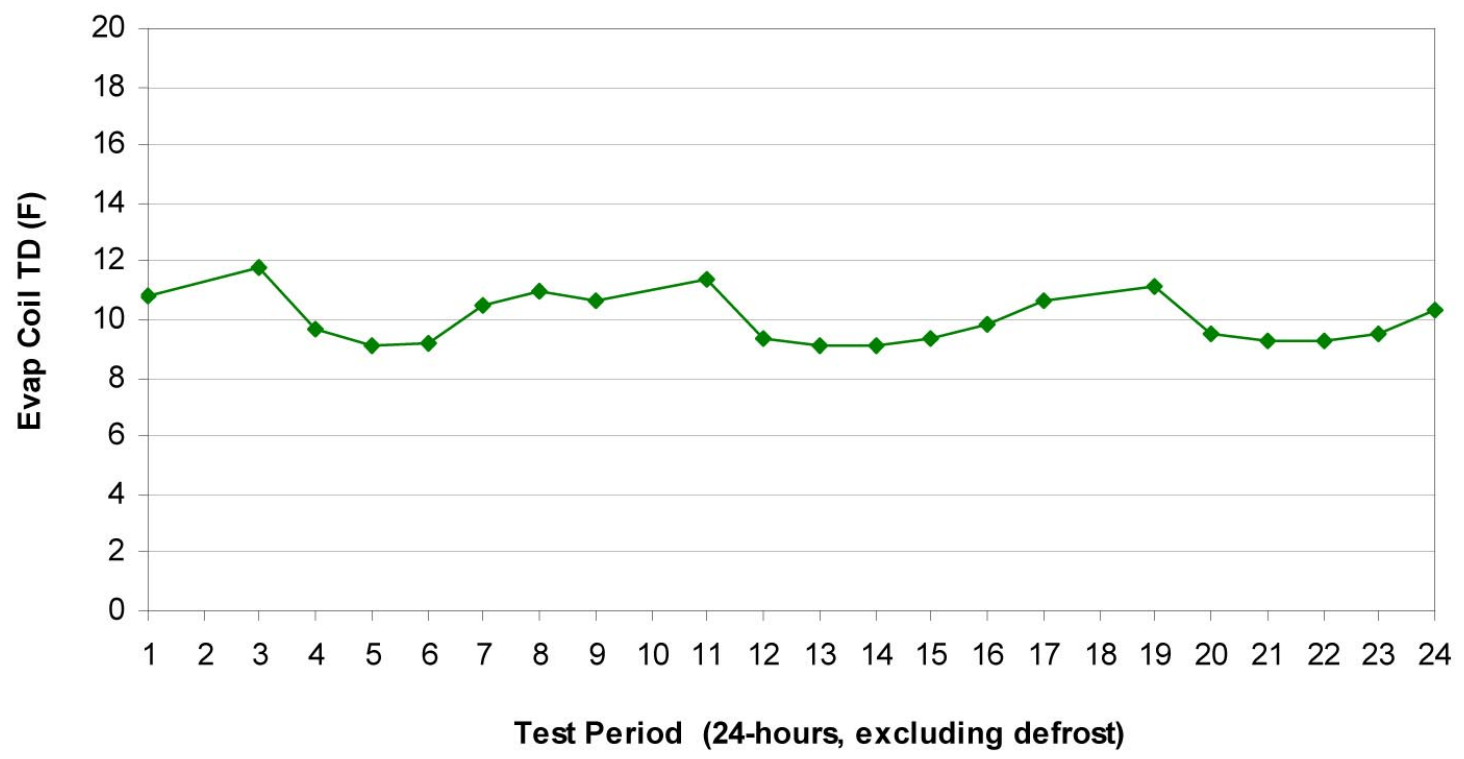

660-DORL-010231-60

Figure 70. Hourly profile of evaporator Coil TD over $24 \mathrm{hr}$

fixed suction pressure, which resulted in maintaining a constant evaporator temperature of $17^{\circ} \mathrm{F}$, coupled with variations in DAT.

The evaporator coil superheat and total system subcooling remained relatively constant during refrigeration periods (Figure 71). However, some variations were observed when the system was approaching defrost and after defrost periods. The average evaporator superheat remained around $10^{\circ} \mathrm{F}$, and average total system subcooling remained around $17^{\circ} \mathrm{F}$.

The hourly profile for the display case's evaporator fan motors, and lighting system power usage is shown in Figure 72. As shown, evaporator fan motors and lighting system power consumption remained unchanged over the entire test period.

Figure 73 depicts the total system power and the total power usage by end use over the entire test period. The fixture's evaporator fan motors power and lighting system power were identical, each consumed about $0.11 \mathrm{~kW}$. The largest contributor to the total system power was the refrigeration system compressor with $1.46 \mathrm{~kW}$. As a result, the total power usage over the entire test period equaled to $1.69 \mathrm{~kW}$.

Additionally, the 2 min profile of product temperatures at six locations inside the display case for each shelf was monitored (Figures 74 through 77). Reviewing Figures 74 through 77 revealed that there was a variation in product temperature profiles depending on the product location, and it varied among shelves. However, the rear products were lower in temperature 


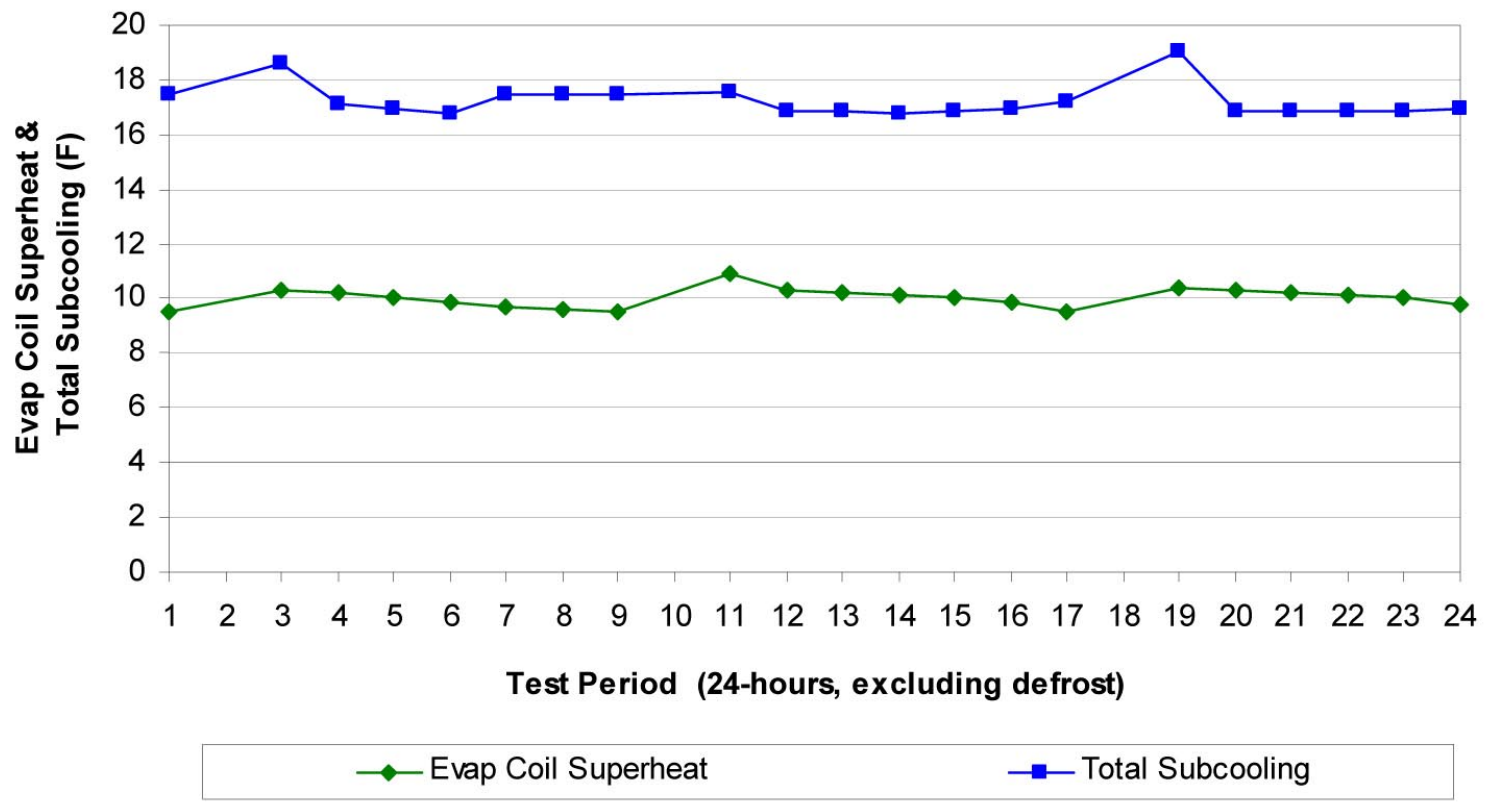

660-DORL-010231-61

Figure 71. Hourly profile of evaporator coil superheat and total system subcooling over $24 \mathrm{hr}$

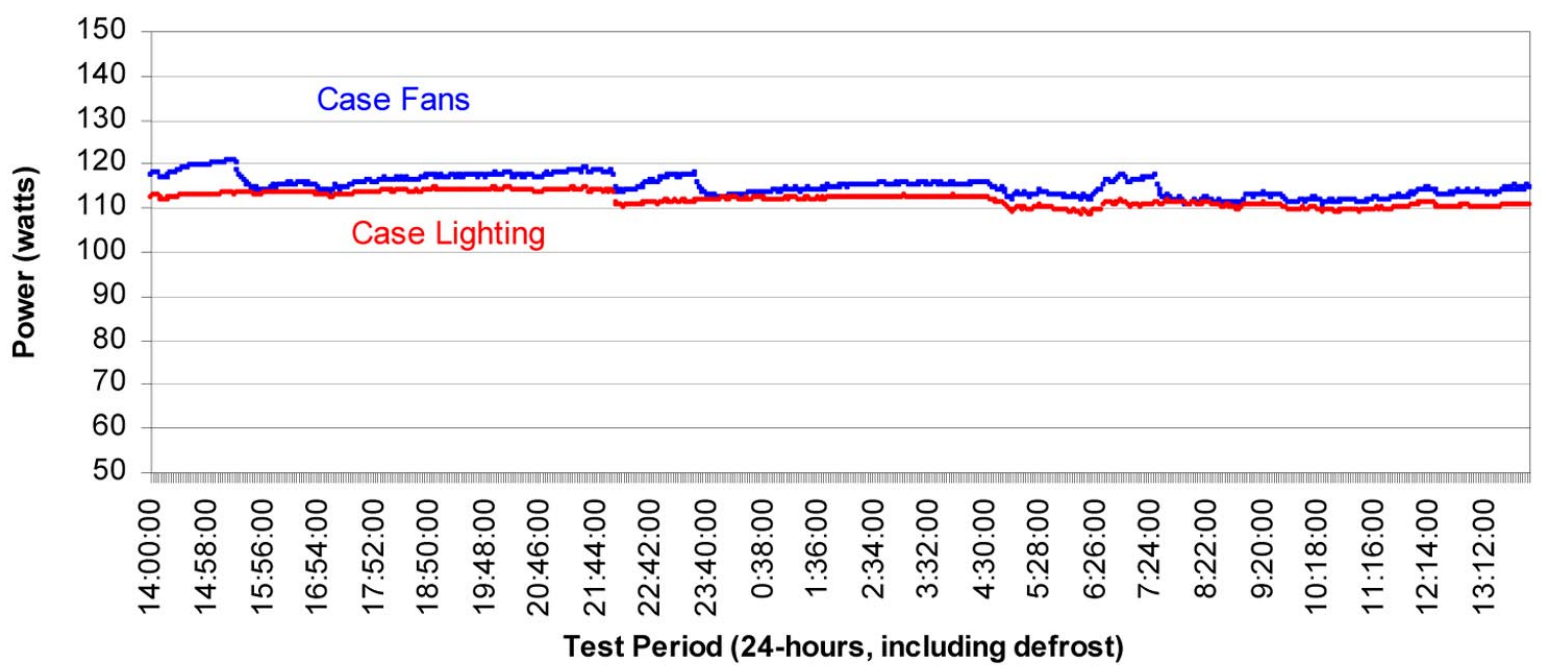

$$
\text { - Case Fans }
$$$$
\text { - Case Lighting }
$$

660-DORL-010231-62

Figure 72. Hourly profile of ASH, lighting, and evaporator fan motor power over $24 \mathrm{hr}$ 


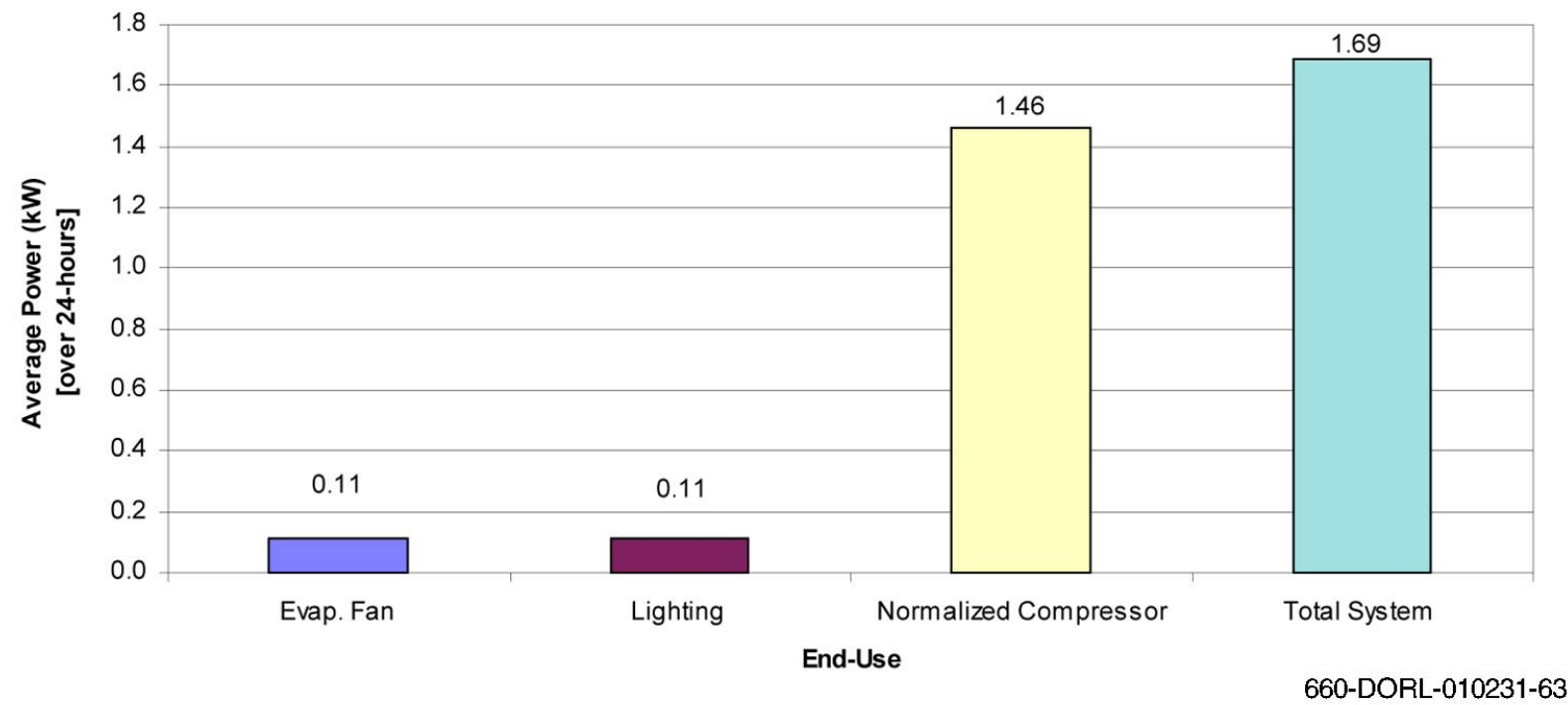

Figure 73. Average total and end-use power over $24 \mathrm{hr}$

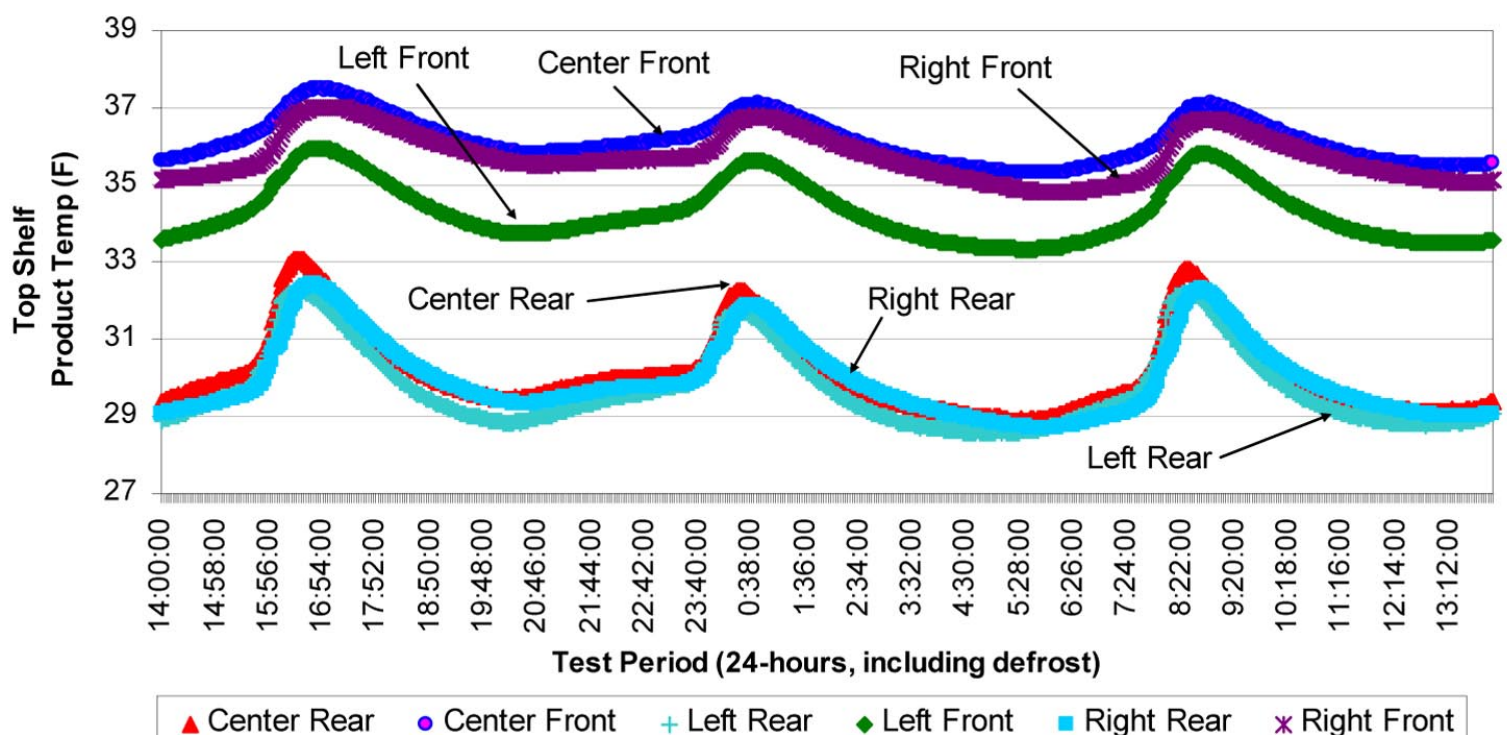

660-DORL-010231-64

Figure 74. 2 min profile of product temperature at six different locations for top shelf over $24 \mathrm{hr}$ 


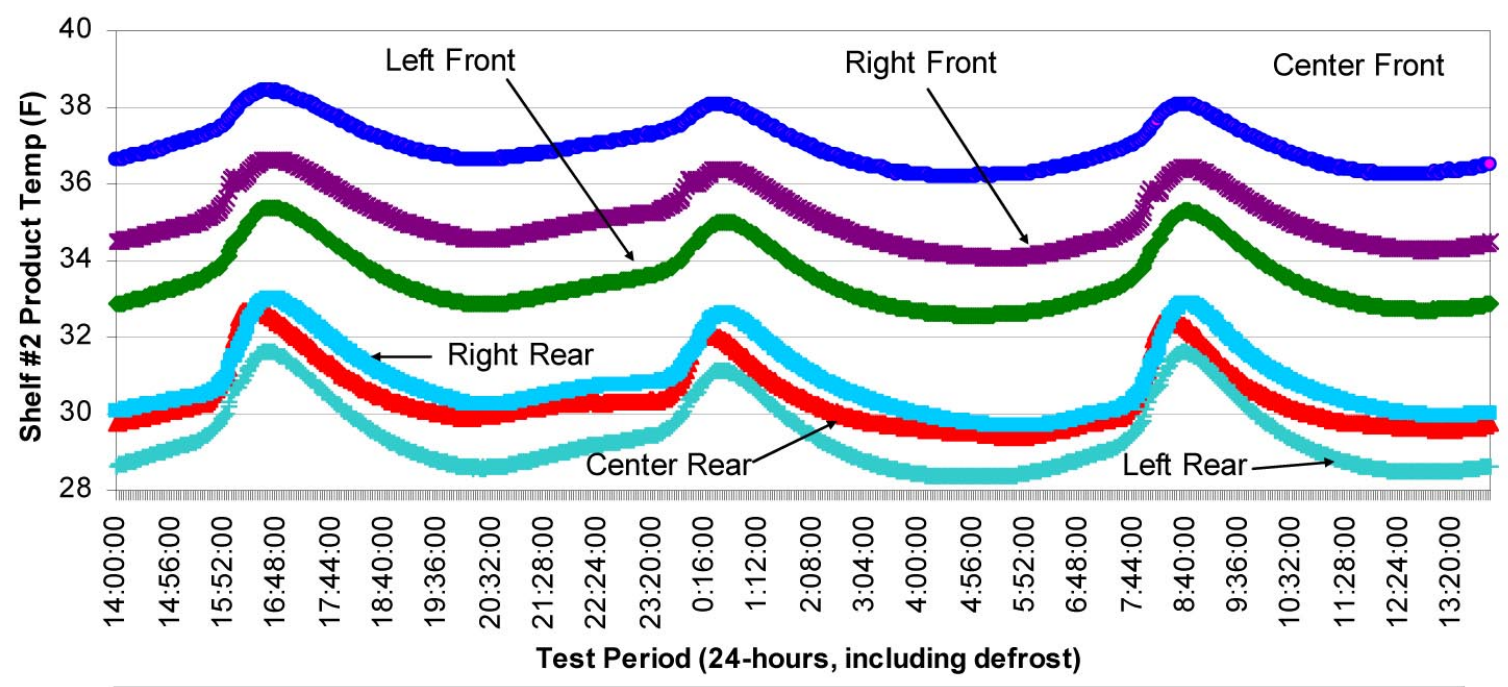

$\Delta$ Center Rear $\quad$ Center Front $\quad+$ Left Rear $\quad$ Left Front $\quad$ Right Rear $\quad *$ Right Front

660-DORL-010231-65

Figure 75. 2 min profile of product temperature at six different locations for second shelf over $24 \mathrm{hr}$

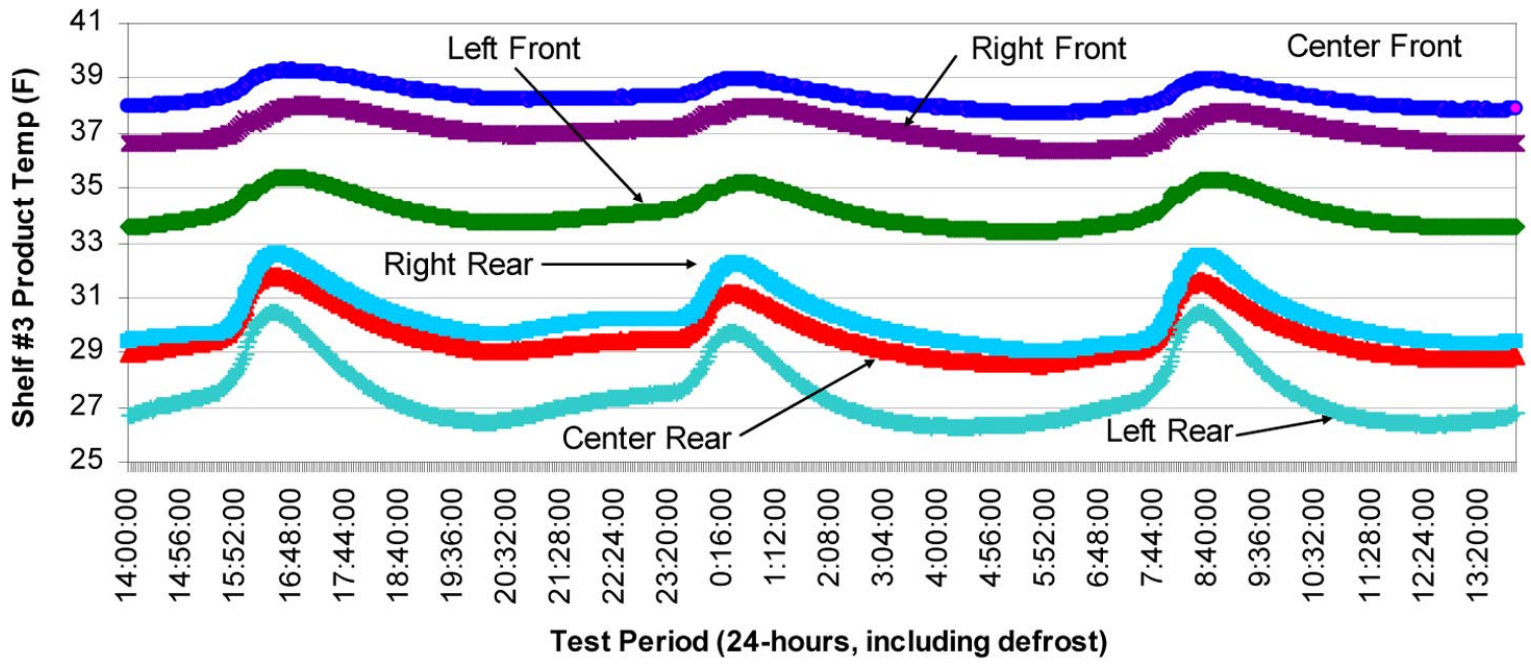

$\Delta$ Center Rear $\quad$ Center Front $\quad+$ Left Rear $\quad$ Left Front $\quad$ Right Rear $\quad *$ Right Front

Figure 76. 2 min profile of product temperature at six different locations for third shelf over $24 \mathrm{hr}$ 


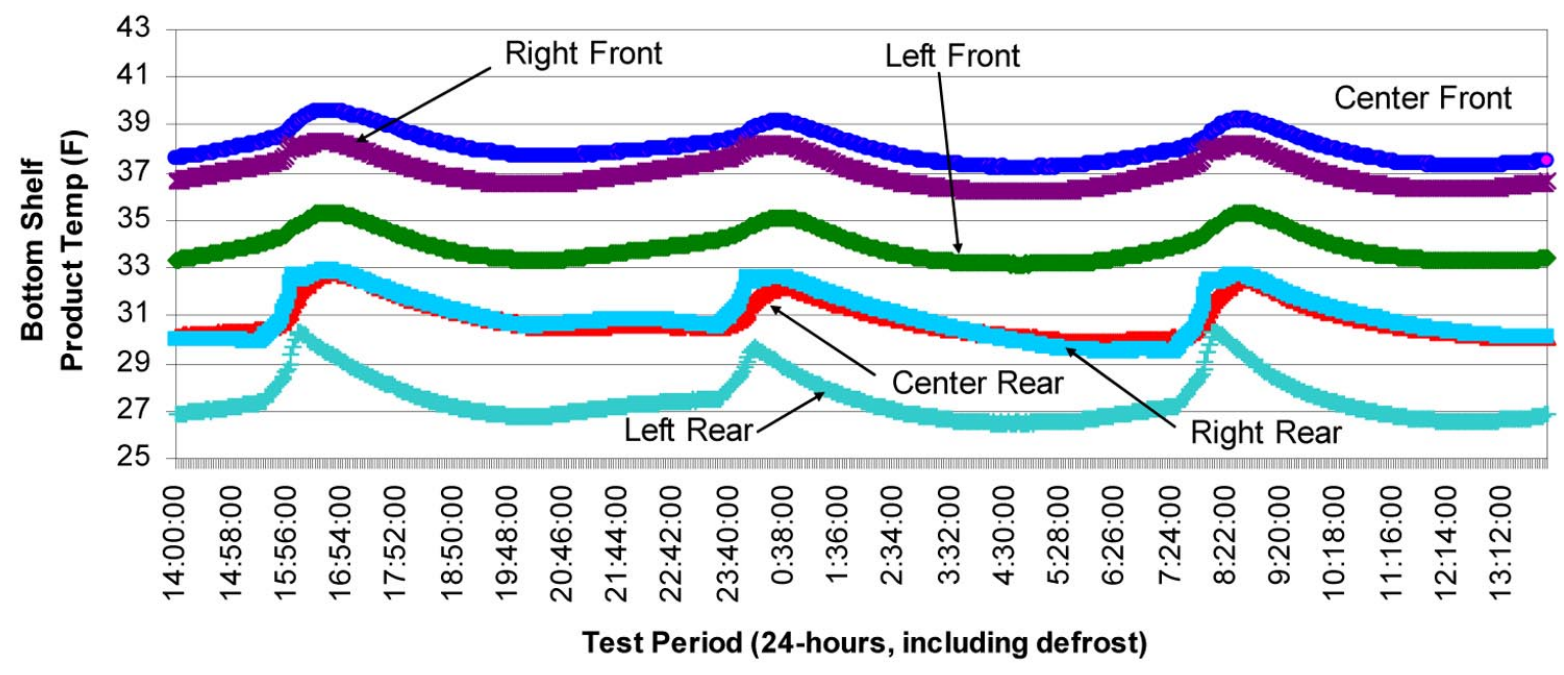

$\Delta$ Center Rear $\bullet$ Center Front $\quad+$ Left Rear $\bullet$ Left Front $\approx$ Right Rear $\quad *$ Right Front

660-DORL-010231-67

Figure 77. 2 min profile of product temperature at six different locations for bottom shelf over $24 \mathrm{hr}$

than the front products, as expected. Also, products located at the left side inside the case were always lower in temperature than those located at the right and center locations.

Figure 78 depicts the average of all six product temperatures for each shelf. As illustrated, the product temperatures were lowest for the top and second shelf. The highest average product temperature was observed for the bottom shelf. Figure 78 also shows that the average product temperatures had similar profiles regardless of variations in temperature magnitudes. The variations in temperature magnitude can be attributed to defrost cycles, and in fact, the products experienced a temperature swing of $2^{\circ} \mathrm{F}$ to $3^{\circ} \mathrm{F}$ as a result.

The coldest product temperature was $26.3^{\circ} \mathrm{F}$ and the warmest was about $39.4^{\circ} \mathrm{F}$ (Figure 79). Average coldest and warmest product temperatures were $27.5^{\circ} \mathrm{F}$ and $38.3^{\circ} \mathrm{F}$, respectively. Averaging all of the product simulators yielded to an average product temperature of $32.8^{\circ} \mathrm{F}$.

In addition, an infrared image of the temperature gradient in front of the display case was captured (Figure 80). The two vertical lines in the figure (in white) illustrate two vertical poles that were located at a horizontal distance of $2 \mathrm{ft}$ (left vertical line) and $4 \mathrm{ft}$ (right vertical line) from the display case. As shown, the air temperatures are considerably lower closer to the display case due to the refrigerated air spillage. The regions in red illustrate the room temperature, which was around $75^{\circ} \mathrm{F}$. 


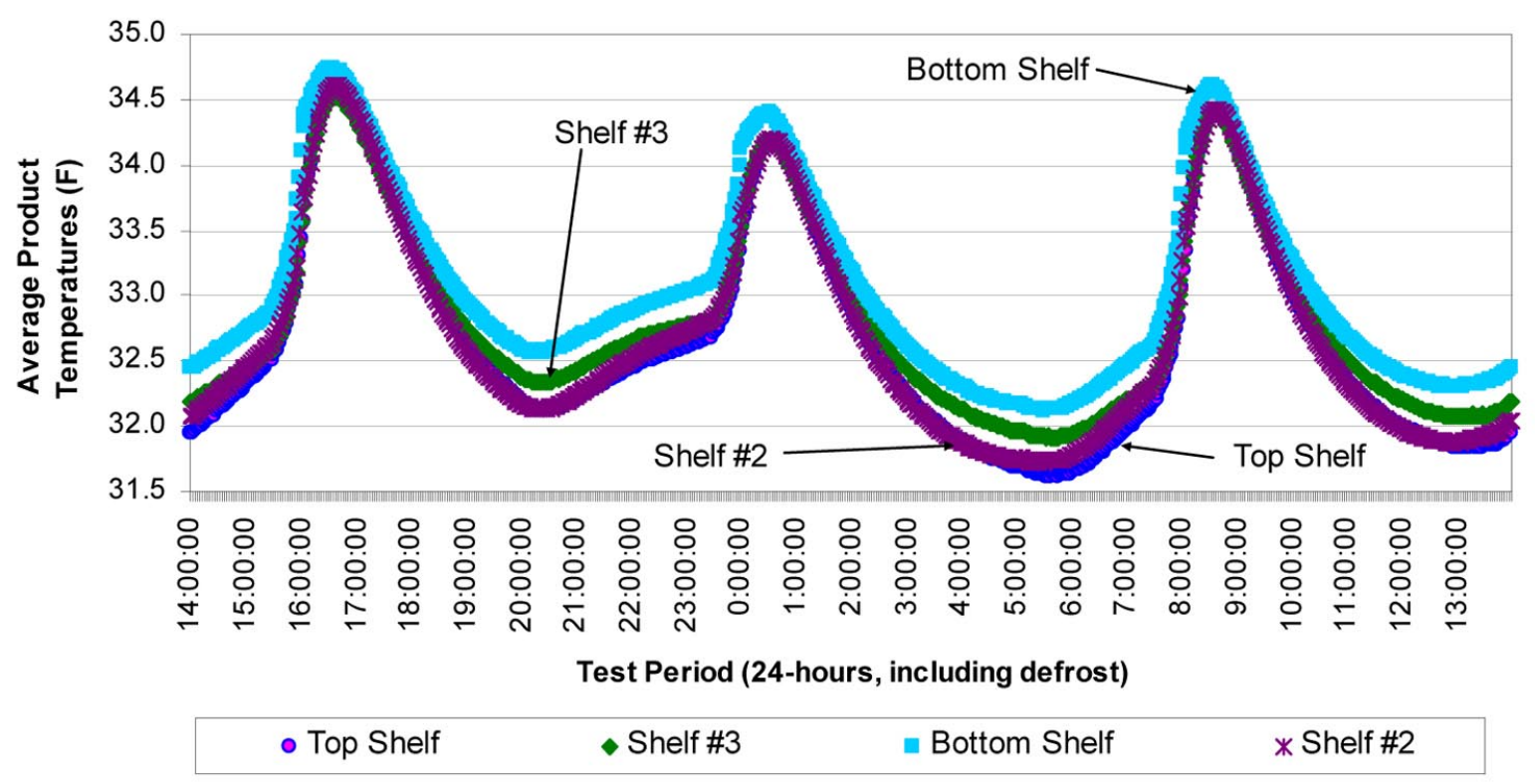

660-DORL-010231-68

Figure 78. Average product temperatures for each shelf over $24 \mathrm{hr}$

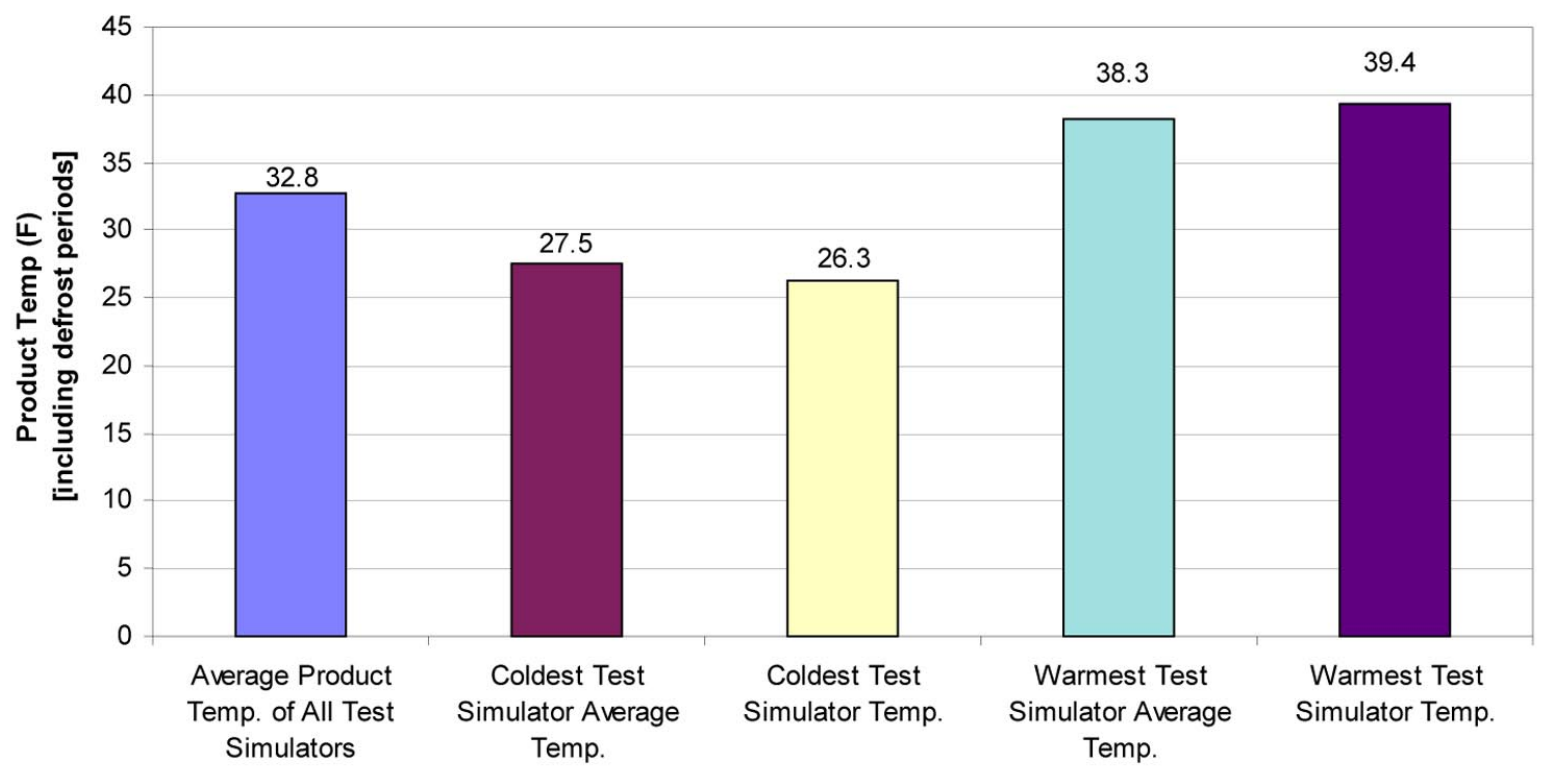

660-DORL-010231-69

Figure 79. Average, coldest and warmest product temperatures over $24 \mathrm{hr}$ 


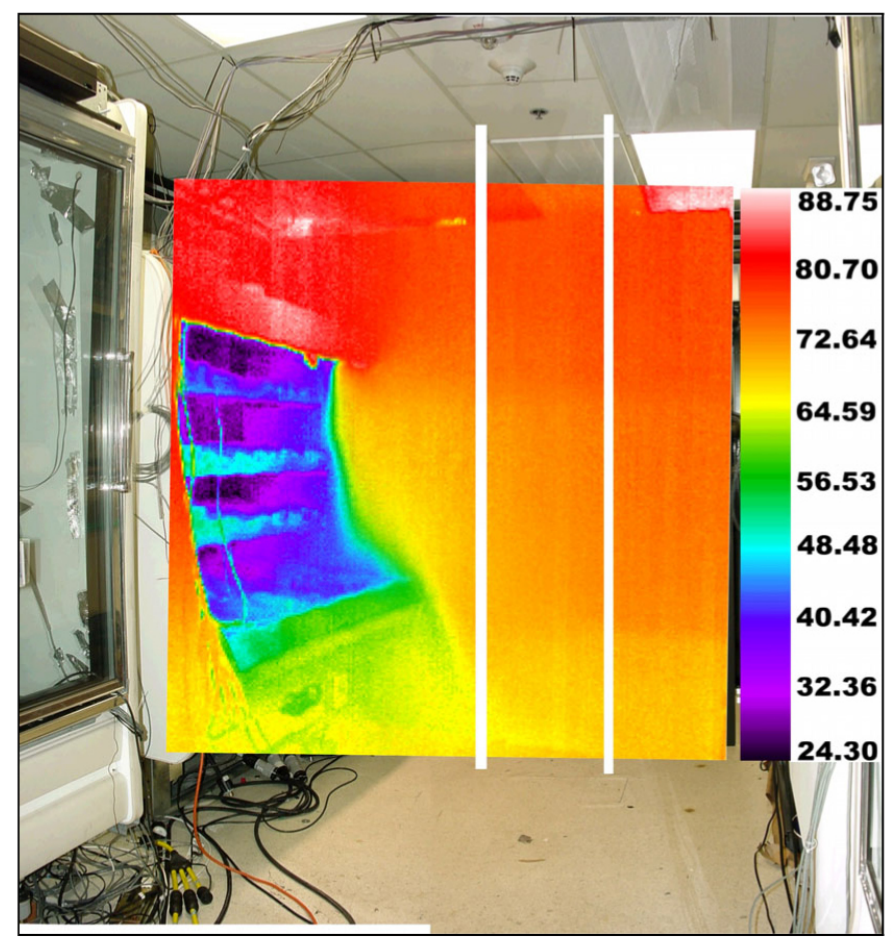

660-DORL-010231-70

Figure 80. Infrared image of temperature gradient in front of the display case

The air temperatures on these two vertical poles were measured in $1 \mathrm{ft}$ increments from the floor in a vertical direction. Figure 81 shows the air temperatures on both vertical poles from $1 \mathrm{ft}$ to $8 \mathrm{ft}$ in a vertical direction. As shown, the air temperature profiles remained identical at $2 \mathrm{ft}$ and $4 \mathrm{ft}$ away from the fixture. Clearly, air temperatures were slightly higher at $4 \mathrm{ft}$ away from the case, and this variation in temperature increased slightly as the vertical distance was increased (from 3 to $8 \mathrm{ft}$ ).

Additionally, the collected test data was compared against the manufacturer's published data. The results are summarized in Table 14. As illustrated, the obtained test results were in close agreement with the manufacturer's published data.

The infiltration rate obtained from the test data was compared to the reported infiltration rate from the CFD model. Figure 82 depicts that both methods yielded a relatively similar result. The CFD model reported only $18 \mathrm{cfm}$ (or 15 percent) lower infiltration rate when compared to the obtained test result.

\subsection{Prototype Test Results}

The prototype display case was fabricated based on the specifications developed in Phase I (Figure 83). The main energy-efficiency features of the prototype case include:

1. Evaporator coil assembly.

2. Air distribution system. 


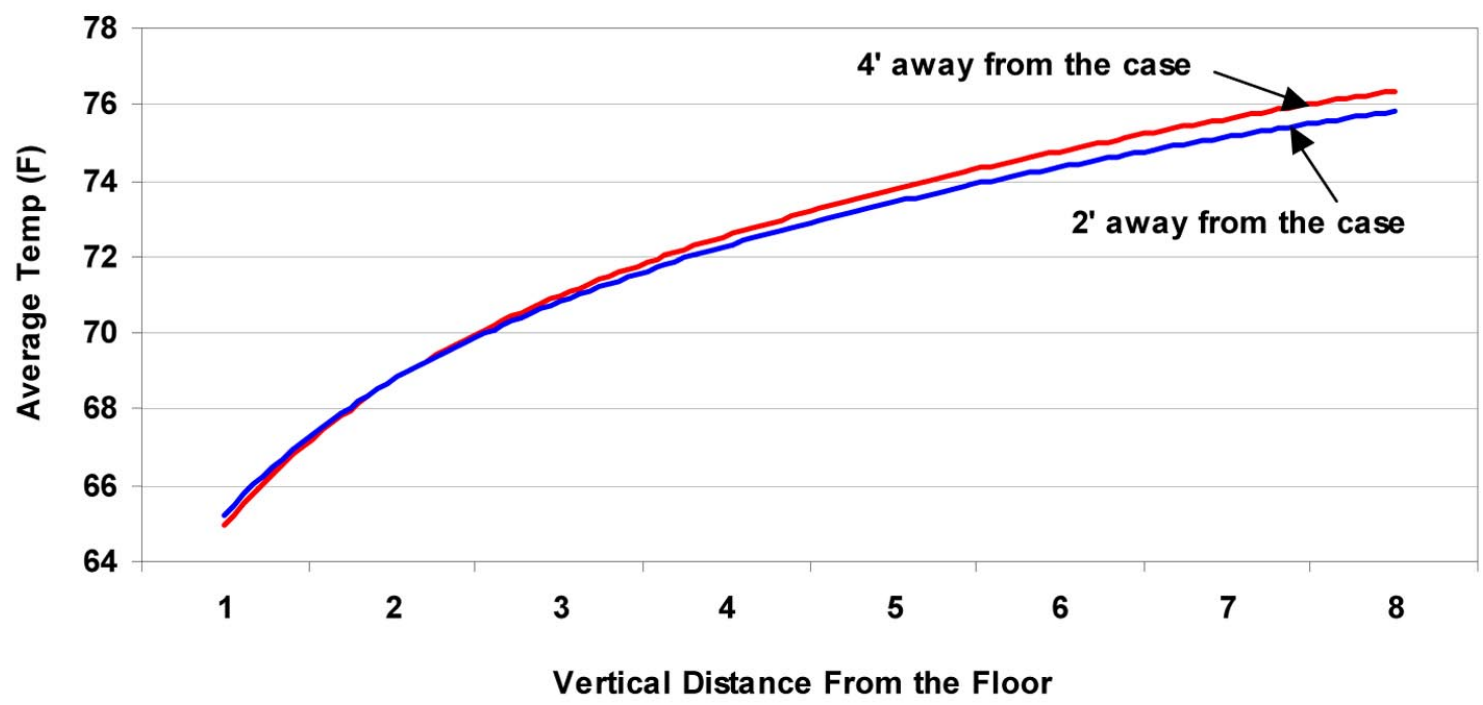

660-DORL-010231-71

Figure 81. Average air temperature at 2 ft and 4 ft away from the display case

Table 14. Comparative summary of test data and manufacturer's published data

\begin{tabular}{|l|c|c|}
\hline & Manufacturer Data & Test Results \\
\hline Average Cooling Load during 3/4 of Run Cycle $(\mathrm{Btu} / \mathrm{h} / \mathrm{ft})$ & 1,561 & 1,624 \\
\hline Average Saturated Evaporator Temperature $\left({ }^{\circ} \mathrm{F}\right)$ & 17 & 17 \\
\hline Average Superheat Set Point $\left({ }^{\circ} \mathrm{F}\right)$ & 6 to 8 & 10 \\
\hline Average Discharge Air Temperature $\left({ }^{\circ} \mathrm{F}\right)$ & 28 & 30 \\
\hline Average Product Temperature $\left({ }^{\circ} \mathrm{F}\right)$ & 35 & 33 \\
\hline Average Return Air Temperature $\left({ }^{\circ} \mathrm{F}\right)$ & 38 & 37 \\
\hline Average Post Defrost Discharge Air Velocity (fpm) & $\mathrm{N} / \mathrm{A}$ & 186 \\
\hline Peak Post Defrost Discharge Air Velocity $(\mathrm{fpm})$ & 350 & 292 \\
\hline
\end{tabular}

3. Liquid-to-suction heat exchanger.

4. Lighting system.

5. Air curtain.

This section discusses results obtained from testing the prototype display case and compares it to the baseline. One of the critical challenges in testing the prototype and baseline display case was to maintain an identical maximum product temperature, while keeping average DAT at $30^{\circ} \mathrm{F}$ $\left( \pm 0.5^{\circ} \mathrm{F}\right)$. As noted in subsections 4.5.2 and 4.5.4, our initial test results of the prototype were obtained with about $2^{\circ} \mathrm{F}$ higher product temperatures than for the baseline case. Based on input received from the industry participants at the January 2004 workshop, the tests were redone with matching product temperatures. The warmest product temperature for the prototype was held to $39.6^{\circ} \mathrm{F}\left( \pm 0.5^{\circ} \mathrm{F}\right)$, which was observed at the bottom shelf front center location and occurred during post-defrost periods. This is essentially equal to that seen in the baseline case tests 


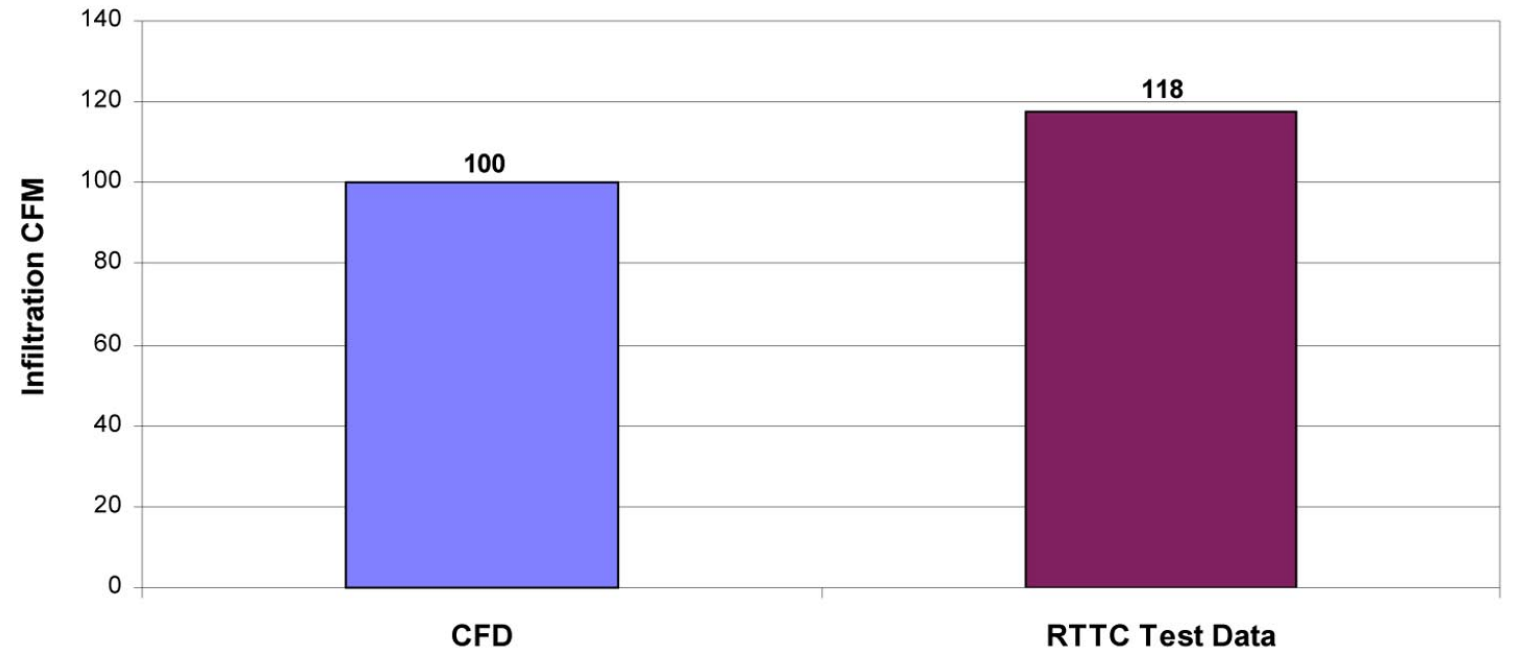

660-DORL-010231-72

Figure 82. Comparison of infiltration rate based on test results and CFD model
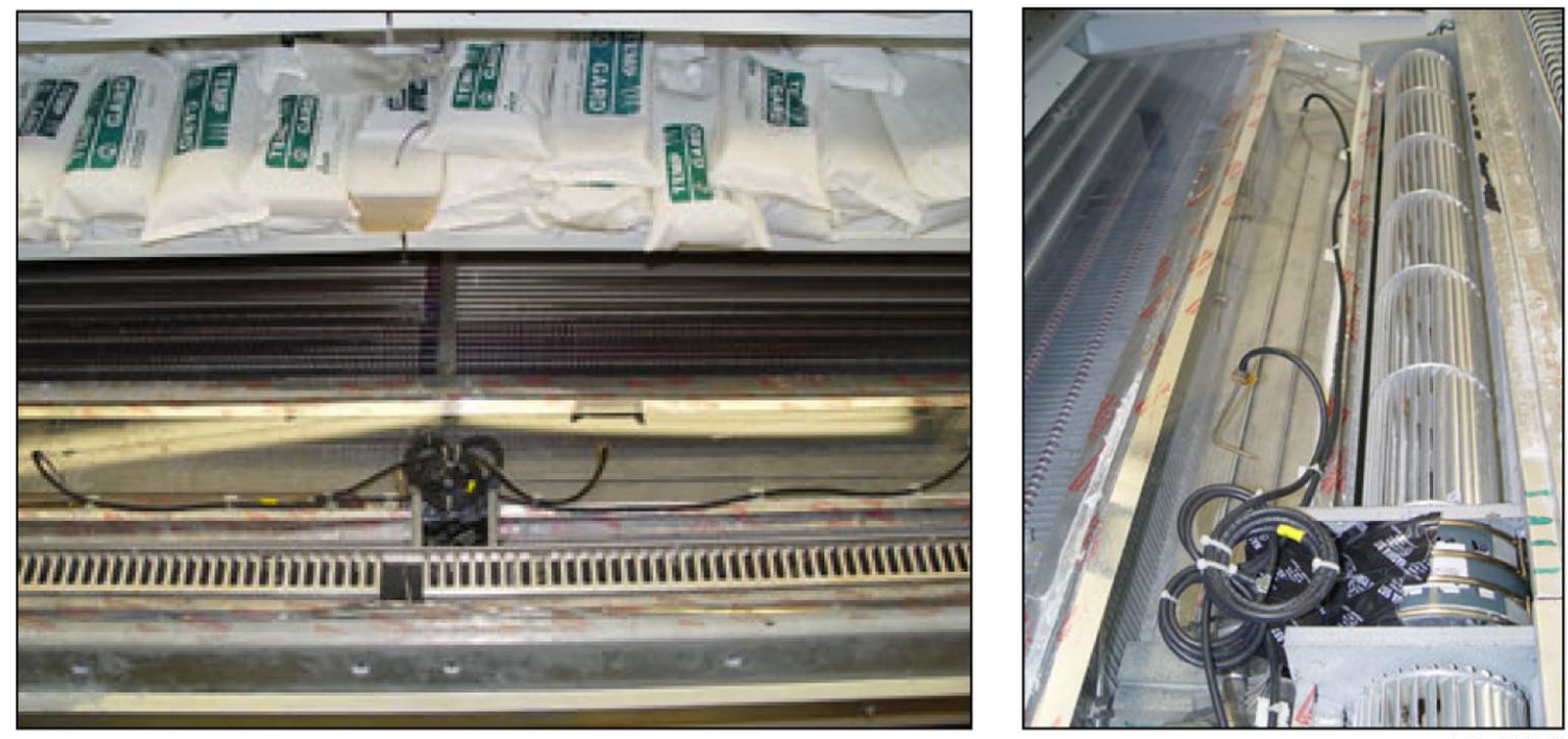

04-1684

Figure 83. Prototype display case showing fan and evaporator coil 
$\left(39.4^{\circ} \mathrm{F} \pm 0.5^{\circ} \mathrm{F}\right)$ (Figure 79$)$. Test results reported in this section are for the case of equal product temperatures.

The refrigeration system, test chamber, and the product temperatures were allowed to reach a steady-state equilibrium condition prior to initiation of the test runs. Figure 84 illustrates that the test chamber maintained relatively non-varying $\mathrm{DB}$ and $\mathrm{RH}$ during the entire test period for both units.

Figure 85 depicts the discharge and suction pressures for both display cases. The test rack controller was programmed to run at a fixed discharge pressure of $220 \mathrm{psig}$, corresponding to $95^{\circ} \mathrm{F} \mathrm{SCT}$, for both test scenarios. For the baseline, the suction pressure was set according to the case manufacturer's specifications to provide a SET of $17^{\circ} \mathrm{F}$. For the prototype case, the SET (or the suction pressure) was raised until similar DAT and maximum product temperature as the baseline were achieved. As a result, the prototype case operated at a SET of roughly $23^{\circ} \mathrm{F}$, which was about $6^{\circ} \mathrm{F}$ (or 8 psig) higher than the baseline case.

\subsubsection{Discharge Air Temperature and Product Temperatures}

The average DAT for both the baseline and prototype cases was about $30^{\circ} \mathrm{F}\left( \pm 0.5^{\circ} \mathrm{F}\right)$ while maintaining an average product temperature of about $33^{\circ} \mathrm{F}$ (Figure 86 ). The highest product temperature for both scenarios was recorded during post-defrost periods while the lowest was prior to initiation of defrost. The variations in product temperature can be attributed to variations in DAT. The DAT reached its lowest temperature at the beginning of the refrigeration periods

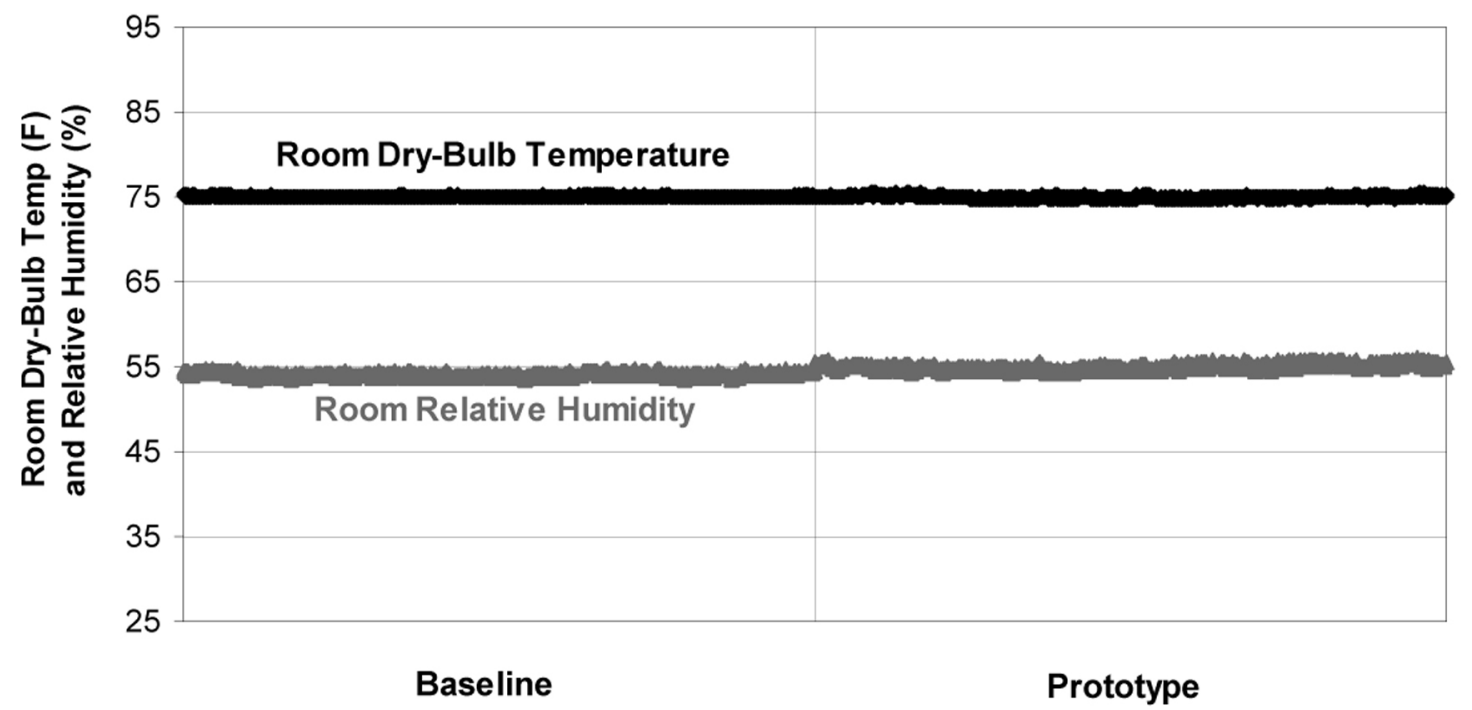

660-DORL-010231-77

Figure 84. 2 min room dry-bulb and relative humidity data over $24 \mathrm{hr}$ period 


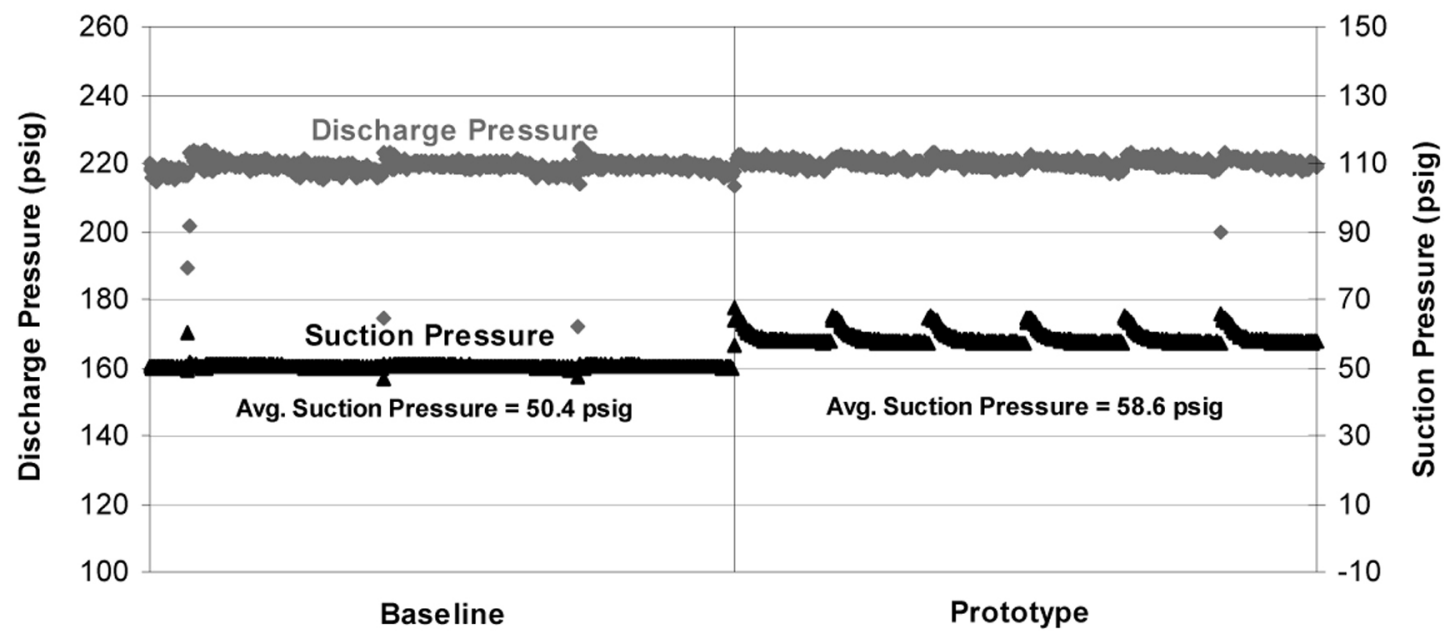

660-DORL-010231-78

Figure 85. 25 min suction and discharge pressures data over 24 hr period

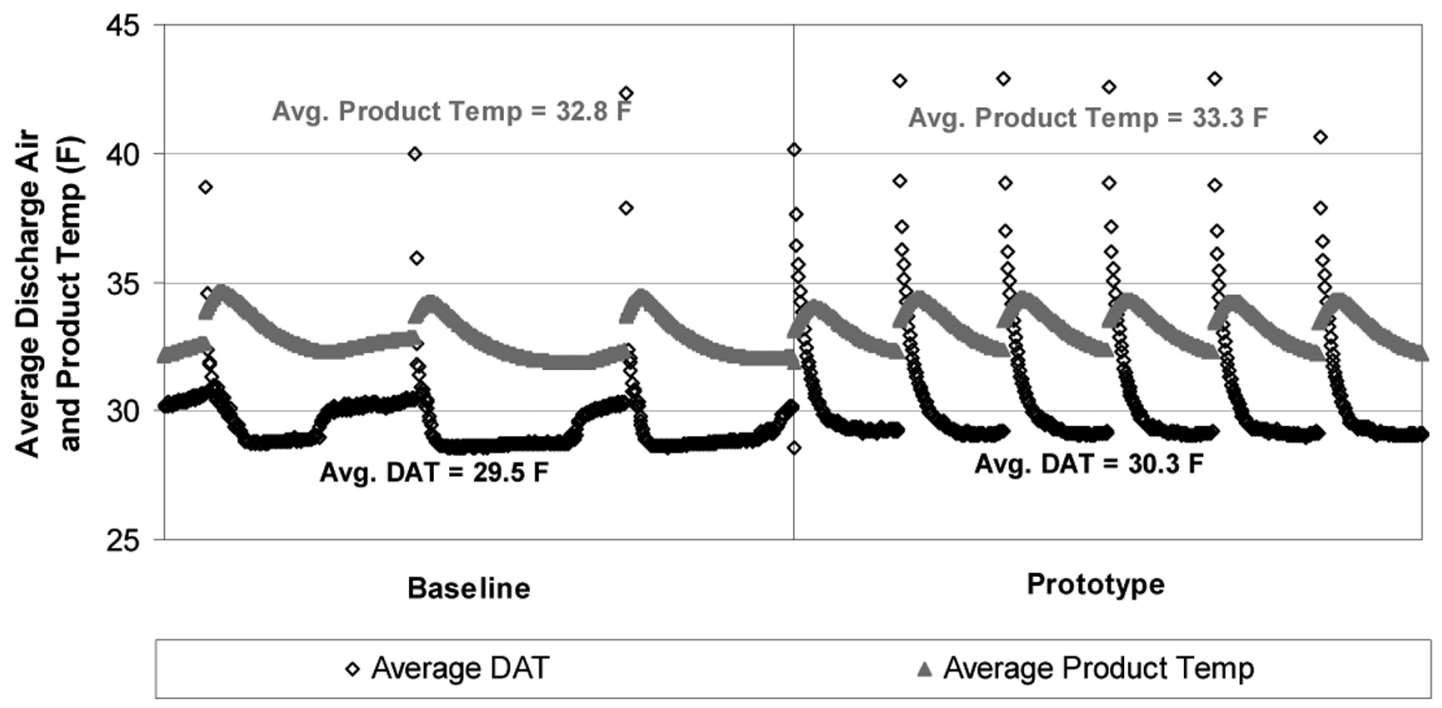

660-DORL-010231-79

Figure 86. 2 min average discharge air temperature and product temperature data over $24 \mathrm{hr}$ period 
due to frost or ice having been removed from the coils during defrosts. Unlike the prototype case, the DAT of the baseline tends to increase almost halfway through refrigeration cycles. This increase can be attributed to larger frost formation on the baseline coil as a result of longer refrigeration periods.

\subsubsection{Collected Condensate and Defrost Duration}

Figure 87 depicts the 2 min profile of condensate mass collected over the entire test period for both display cases. As shown, the overall magnitudes of collected condensate were relatively close for both scenarios. Horizontal lines in Figure 87 show no (or insignificant amount of) moisture being collected during refrigeration periods. Vertical lines indicate the mass of melted frost collected during each defrost cycle.

The defrost frequency for the baseline case was set to three defrosts per day (based on the manufacturer's specifications), which resulted in $102 \mathrm{~min}$ of defrost over $24 \mathrm{hr}$. With only three defrosts per day, the more effective evaporator of the prototype case experienced faster frost blockage due to its higher rate of moisture removal than the baseline. As a result, after conducting a series of experiments, the optimum number of defrost cycles was set to six per day to minimize the adverse post-defrost product temperature rise. Doubling the number of defrost cycles in the prototype case increased the overall defrost duration to $168 \mathrm{~min}$.

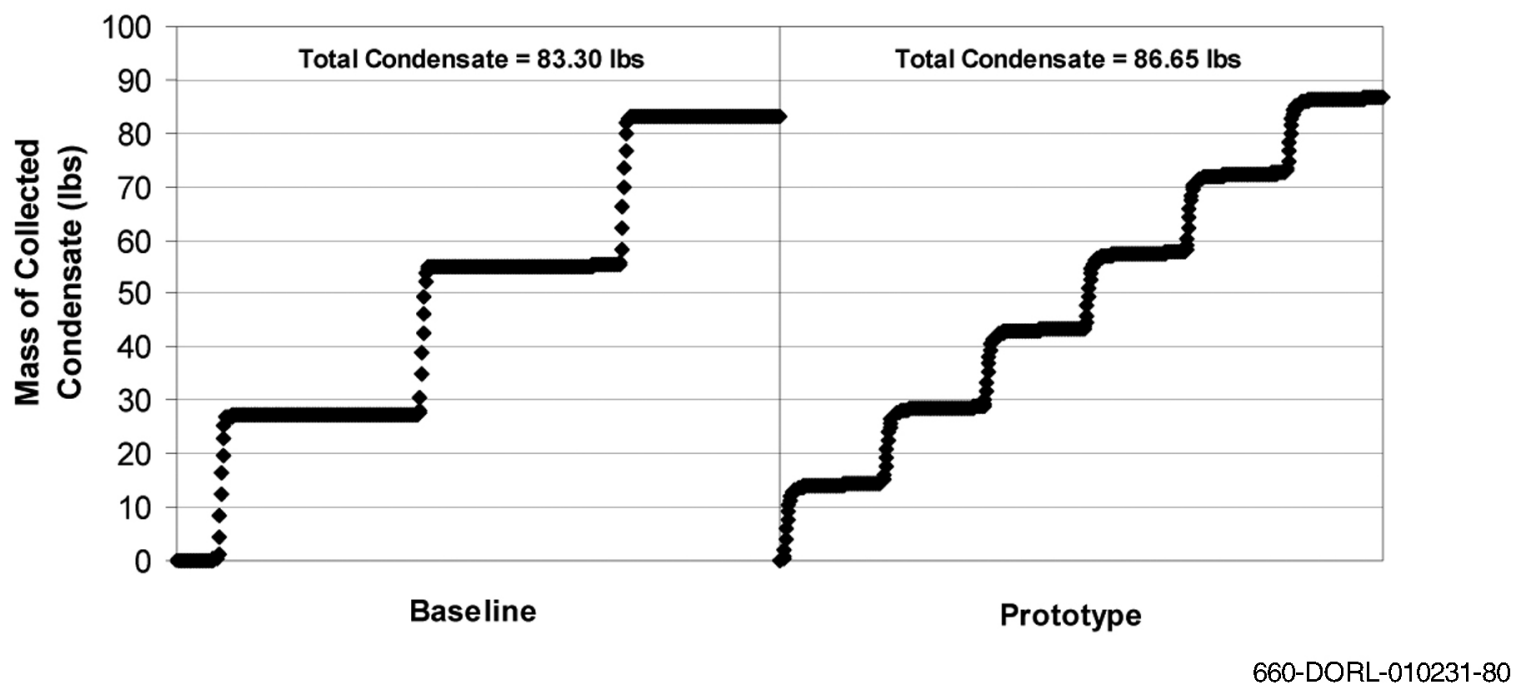

Figure 87. 2 min mass of collected condensate data over $24 \mathrm{hr}$ period 


\subsubsection{Air Curtain Performance}

The CFD results from the air curtain study (Section 8) suggested that DAV characteristics including velocity profile, Reynolds number and turbulence intensity have significant impacts on infiltration. The CFD model predicted the lowest entrainment rate for the DAG design and Reynolds number described as the $1^{\text {st }}$ generation air curtain redesign (subsection 8.4.2) that was incorporated into this prototype case. Unfortunately, this design failed to yield any significant reductions in the infiltration rate or total cooling load.

\subsubsection{Evaporator Coil Effectiveness, Superheat, and Total Subcooling}

The evaporator effectiveness as a function of the product of overall coefficient of heat transfer and surface area (UA) for the prototype was improved by 68 percent over the baseline case. Using a micro-channel LSHX resulted in roughly a $34^{\circ} \mathrm{F}$ increase in total system subcooling. The prototype case used a dual port mechanical TXV. This allowed for faster temperature recovery than in the baseline case during post-defrost periods. It also allowed for more effective utilization of the evaporator by operating at $6^{\circ} \mathrm{F}$ superheat, which was $4^{\circ} \mathrm{F}$ less than the baseline.

\subsubsection{Refrigeration Effect and Refrigerant Mass Flow Rate}

Figure 88 depicts the refrigeration effect and refrigerant mass flow rate for both cases. Refrigeration effect represents the cooling capacity of the evaporator per pound of refrigerant

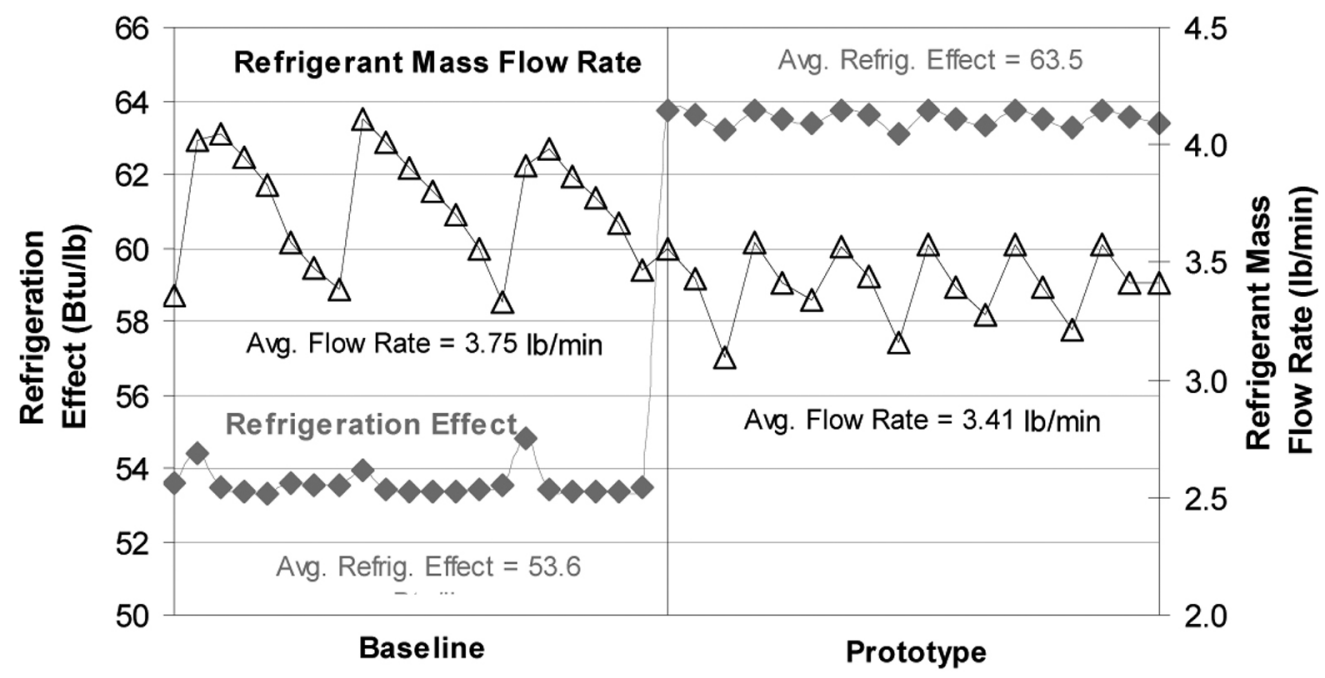

660-DORL-010231-81

Figure 88. 2 min refrigeration effect and refrigeration mass flow rate data over 24 hr period 
flow. The prototype case was operated at $63.5 \mathrm{Btu} / \mathrm{lb}$, which was $10 \mathrm{Btu} / \mathrm{lb}$ (or 18 percent) higher than the baseline. This improvement was largely due to the use of a more effective evaporator, which allowed for operations at higher SET. High-efficiency LSHX, which increased subcooling from $17^{\circ} \mathrm{F}$ to $52^{\circ} \mathrm{F}$ also made a partial contribution to refrigeration effect improvement. The prototype case also required 9 percent less circulation of refrigerant than the baseline, which was a result of the improved refrigeration effect.

\subsubsection{Cooling Capacity and Cooling Load Components}

The cooling capacity, which is a function of refrigeration effect and refrigerant mass flow rate as defined in subsection 9.1.2, was $12,989 \mathrm{Btu} / \mathrm{hr}$ and 12,977 $\mathrm{Btu} / \mathrm{hr}$ for the baseline and the prototype, respectively. The prototype case experienced 2 percent higher infiltration load (computed as outlined in subsection 9.2.4) than the baseline (10,835 versus $10,674 \mathrm{Btu} / \mathrm{hr})$. The increase in infiltration load of the prototype case can be attributed to modifications made to the DAG configuration. The total cooling load was comprised of infiltration, radiation, conduction, and internal loads (lights and evaporator fans). As expected, the largest component of the cooling load was infiltration, which contributed to over 80 percent of the total cooling load for each display case. Based on the heat transfer model's results, the contribution of radiation (8 percent) and conduction (4 percent) to the total cooling load remained unchanged since both display cases operated at similar average DAT. The contribution of internal loads, however, decreased from 6 percent in the baseline case to 5 percent in the prototype. This reduction was due to less heat dissipation from the high-efficiency lighting system and evaporator fan motor.

\subsubsection{Power, Energy, and System Efficiency}

The prototype unit required roughly 20 percent less fan and lighting power than the baseline unit (Figure 89). Furthermore, the increase in refrigeration effect and decrease in refrigerant mass flow resulted in a drop of 10 percent in the compressor power requirement of the prototype case. Combining the savings obtained from the compressor, evaporator fan motor and lighting yielded 11 percent reduction in total power consumption. The decrease in total power requirements of the prototype case improved its energy efficiency. The energy-efficiency ratio (EER), which is defined as a ratio of total cooling load (Btu/hr) to sum of compressor, lights and fan motor power (watts), was increased by 22 percent for the prototype case. Additionally, the decrease in compressor power run time of the prototype case translated into a saving of 14 percent in compressor energy usage.

In summary, the prototype system energy usage improved while maintaining target product temperatures. Table 15 summarizes and compares the key performance attributes of both the prototype and baseline cases. As shown, the energy savings achieved by the prototype display case was $6 \mathrm{kWh} /$ day, which was 15 percent of the daily energy consumption of the baseline display case. 


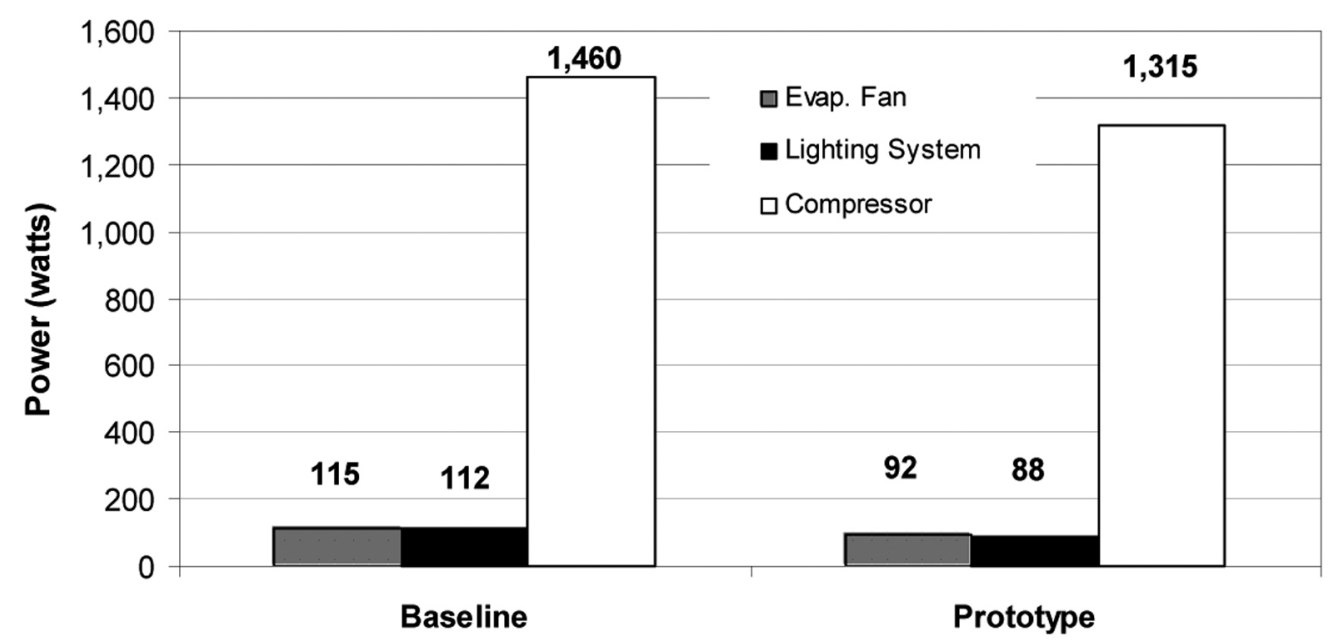

660-DORL-010231-82

Figure 89. Average end-use power data over $24 \mathrm{hr}$ period

Table 15. Comparison of key performance attributes of baseline and prototype cases

\begin{tabular}{|l|c|c|c|}
\hline & Baseline & Prototype & Difference \\
\hline SET $\left({ }^{\circ} \mathrm{F}\right)$ & 17 & 23 & $+6{ }^{\circ} \mathrm{F}$ \\
\hline UA (Btu/hr- $\left.{ }^{\circ} \mathrm{F}\right)$ & 934 & 1,567 & $-68 \%$ \\
\hline Superheat $\left({ }^{\circ} \mathrm{F}\right)$ & 10.0 & 5.6 & $-4.4{ }^{\circ} \mathrm{F}$ \\
\hline Subcooling $\left({ }^{\circ} \mathrm{F}\right)$ & 17.2 & 51.6 & $+34.4{ }^{\circ} \mathrm{F}$ \\
\hline Refrigeration Effect (Btu/lb) & 53.6 & 63.5 & $-19 \%$ \\
\hline Refrigerant Mass Flow (lb/min) & 3.75 & 3.41 & $9 \%$ \\
\hline Total Refrigeration Load (Btu/hr) & 12,989 & 12,977 & - \\
\hline Conduction Load (Btu/hr) & 526 & 514 & $2 \%$ \\
\hline Radiation Load (Btu/hr) & 1,016 & 1,014 & $0.2 \%$ \\
\hline Internal (lights and fans) Load (Btu/hr) & 773 & 614 & $21 \%$ \\
\hline Infiltration Load (Btu/hr) & 10,674 & 10,835 & $-2 \%$ \\
\hline Mass of Condensate Collected over 24 hr $(\mathrm{lb})$ & 83.30 & 86.65 & $-4 \%$ \\
\hline Compressor Power $(\mathrm{W})$ & 1,460 & 1,315 & $10 \%$ \\
\hline Compressor Energy (kWh) & 32.6 & 27.9 & $14 \%$ \\
\hline Fan Motor $(\mathrm{W})$ & 115 & 92 & $20 \%$ \\
\hline Lighting $(\mathrm{W})$ & 112 & 88 & $21 \%$ \\
\hline Total System Power $(\mathrm{W})$ & 1,687 & 1,495 & $11 \%$ \\
\hline Total System Energy $(\mathrm{kWh})$ & 38.0 & 32.2 & $15 \%$ \\
\hline EER $($ Btu/hrM) & 7.12 & 8.65 & $-22 \%$ \\
\hline Maximum Product Temperature $\left({ }^{\circ} \mathrm{F}\right)$ & 39.4 & 39.6 & $+0.2{ }^{\circ} \mathrm{F}$ \\
\hline
\end{tabular}




\section{DEVELOPMENT OF A DRAFT RATING PROCEDURE FOR HIGH- EFFICIENCY REFRIGERATED DISPLAY CASES}

The purpose of the efficiency rating procedure is to provide a methodology to identify display cases that help promote the efficient use energy in supermarket refrigeration. The rating procedure utilizes industry standards as the basis of comparison and sets an energy savings goal for each candidate display case. Display cases meeting or exceeding the goal are considered "energy-efficient."

The rating procedure looks at both the configuration and application of each display case. Refrigerated display cases used in supermarkets generally fall within 4 configurations, which are:

- Tub.

- Multi-deck.

- Glass door, reach-in.

- $\quad$ Single-deck or service.

Each of these configurations has unique operating characteristics, particularly the make-up of their refrigeration loads, or requirements. The open, multi-deck display cases have a large portion of their requirements attributable to ambient air entrainment in the display case air curtain. The requirement of the single-deck display case has a significant component that is due to thermal radiation with the surrounding environment. The refrigeration load of glass door cases is largely due to the use of anti-sweat heaters in the doors that prevent condensation on the glass. The resulting refrigeration requirement for each of these display case configurations is different. Similarly, lighting, heater, and fan, requirements will vary with case configuration. The rating procedure must, therefore, compare like case configurations in order to determine energy savings.

The type of food product stored in the case determines the application of a display case. The discharge air and evaporator temperatures must be specified to maintain the correct product temperature for each stored food type. Evaporator temperature needed to maintain the desired discharge air temperature impacts the resulting compressor saturated suction temperature, which, in turn, affects the compressor energy consumption. A requirement of the rating procedure is that the candidate display case must be capable of maintaining appropriate product temperature for the application that is specified for the display case. Table 16 gives typical values of discharge air and evaporator temperature for specific storage applications. 
Table 16. Description of display case applications

\begin{tabular}{|l|c|c|}
\hline \multicolumn{1}{|c|}{ Application } & $\begin{array}{c}\text { Discharge Air Temperature } \\
\left({ }^{\circ} \mathrm{F}\right)\end{array}$ & $\begin{array}{c}\text { Evaporator Temperature } \\
\left({ }^{\circ} \mathrm{F}\right)\end{array}$ \\
\hline Ice Cream & -10 & -20 \\
\hline Frozen Food & -5 & -15 \\
\hline Red Meats & 22 & 12 \\
\hline Meat/Deli & 28 & 17 \\
\hline Fish & 28 & 17 \\
\hline Dairy & 32 & 21 \\
\hline Produce & 32 & 24 \\
\hline Beverage & 35 & 27 \\
\hline
\end{tabular}

The rating procedure takes into account both the case configuration and application. Cases of like configuration and application are compared to determine energy savings of candidate cases. Examples include case descriptions such as multi-deck dairy, single-deck meat, and reach-in frozen food. Descriptions for existing display cases can be found readily in the product data provided by the major display case manufacturers. Some specialty display cases do exist that will have to be dealt with individually.

The significant information needed to rate a candidate display case is the following:

- Refrigeration Load or Requirement - The amount of heat energy that must be removed by the refrigeration system in order to maintain the display case at the desired discharge air temperature. The procedures to measure the refrigeration requirement for remotely refrigerated display cases are given in the ASHRAE standards 72 and $117(\underline{6}, \underline{7})$, for open and closed cases, respectively. Display case manufacturers follow these standards and the refrigeration requirements for each display case configuration and application can be found in the resulting product data and specifications.

- Discharge Air Temperature - The temperature of the air leaving the discharge air curtain of the display case. The discharge air temperature is an indicator of the product temperature that can be maintained by that display case. The value of the discharge air temperature of the candidate display case should follow the guidelines given in Table 16 for the designated application of the candidate case.

- $\quad$ Saturated Evaporator Temperature (SET) - The temperature at which the display case evaporator operates to remove heat energy from the cases and maintain the discharge air temperature. The SET is also determined from measurements outlined in the ASHRAE standards and is specified by the manufacturer for each case.

- Case Lighting Power - The power draw of the lighting in the display case. The amount of lighting used in a case is normally specified in terms of the number of rows of fluorescent bulbs installed. The number of rows of lights varies depending upon the amount of product illumination desired. The lighting power used in the rating procedure should correspond to the amount of lighting installed in the case when the rated 
refrigeration requirement was established. For lesser or greater amounts of lighting, the refrigeration requirement of the case should be adjusted to compensate for increased heat load from the lights and the lighting power should account for all lights installed.

- Fan Power - The power needed to circulate air within the display case. Several different fan motor types exist including shaded-pole (or standard), split capacitor, or electronically commutated (ECM). The type of fan motor used in the refrigeration requirement measurement should be used in the rating procedure.

- Anti-Sweat Heater Power - The power needed by all anti-sweat heaters used in the case.

The procedure used to estimate the refrigeration energy consumption of the display case is described in detail in ARI standard $1200(\underline{8})$. The procedure given in the standard results in a calculated daily energy consumption (CDEC) of the display case, expressed in terms of $\mathrm{kWh} /$ day/ft of display case length (for glass door reach-in cases, the energy consumption is expressed as $\mathrm{kWh} /$ day/door). The CDEC is found with the following relation

$$
\mathrm{CDEC}=\mathrm{CEC}+\mathrm{FEC}+\mathrm{LEC}+\mathrm{AEC}+\mathrm{DEC}
$$

where

$$
\begin{aligned}
& \text { CDEC }=\text { Calculated Daily Energy Consumption }(\mathrm{kWh} / \text { day/ft }) \\
& \mathrm{CEC}=\text { Compressor Energy Consumption } \\
& \mathrm{FEC}=\text { Fan Energy Consumption } \\
& \mathrm{LEC}=\text { Lighting Energy Consumption } \\
& \mathrm{AEC}=\text { Anti-sweat Heater Energy Consumption } \\
& \mathrm{DEC}=\text { Defrost Energy Consumption }
\end{aligned}
$$

The compressor energy consumption (CEC) is found from the following expression

$$
\mathrm{CEC}=\frac{\left[\left(\mathrm{Q}_{\mathrm{rt}} / \mathrm{L}\right) *\left(\mathrm{t}-\mathrm{t}_{\mathrm{dt}}\right)\right]}{(\mathrm{EER} * 1000)}
$$

where

Qrt = Refrigeration requirement (ASHRAE testing) $(\mathrm{Btu} / \mathrm{hr})$

$\mathrm{L}=$ Length $(\mathrm{ft})$

$\mathrm{t}=24 \mathrm{hr}$

$\mathrm{t}_{\mathrm{dt}}=$ Defrost time $(\mathrm{hr})$

EER = Energy Efficiency Ratio of the Compressor (Btu/W-hr)

The EER value is found in ARI 1200 and is based upon an adjusted saturated evaporator temperature that is equal to the rated saturated evaporator temperature minus $3^{\circ} \mathrm{F}$. Figure 90 provides a graph of these EERs. Defrost time is the total time the case spends in defrost over 


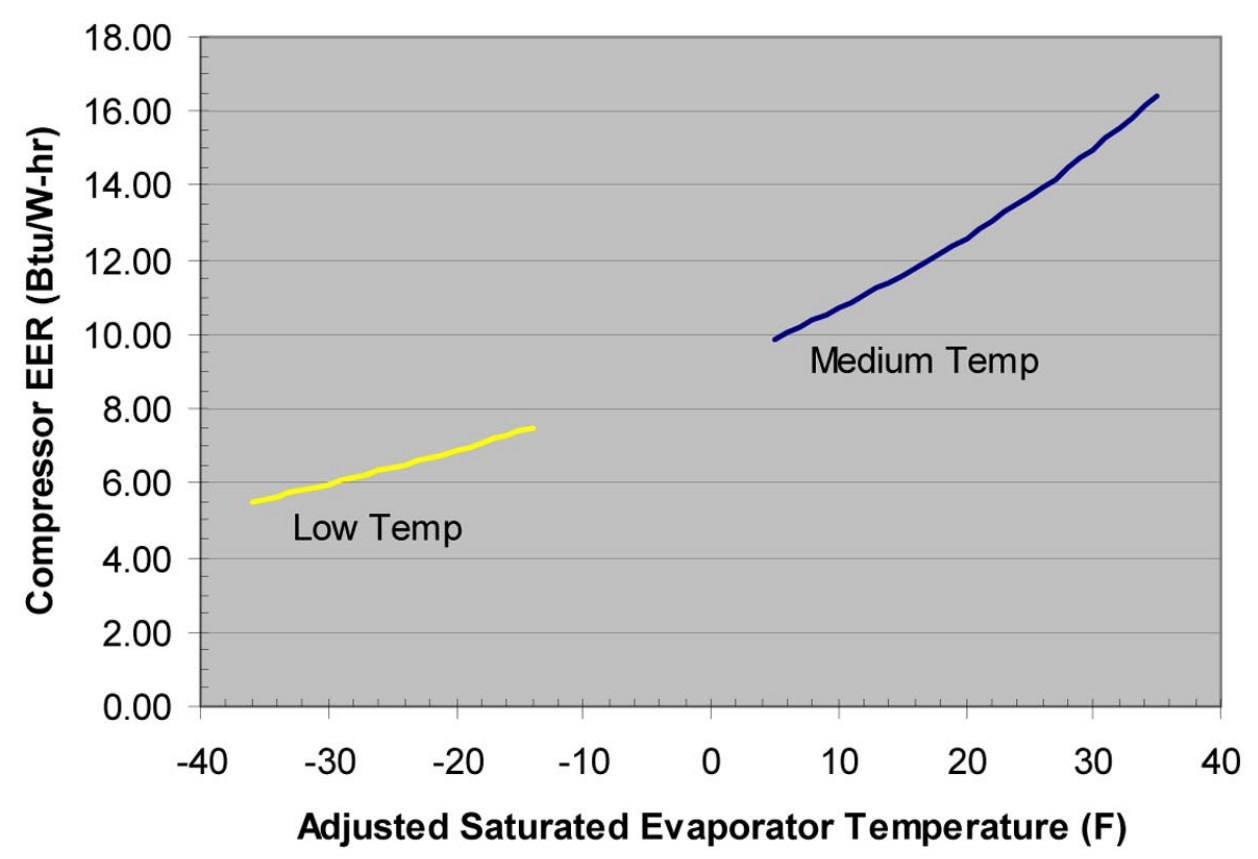

660-DORL-010231-75

Figure 90. Compressor EER values for ARI 1200 energy consumption calculation

$24 \mathrm{hr}$ and is the product of the recommended number of defrosts per day and duration of each defrost.

The remaining energy terms consist of the rated power values for each component (lights, fans, heaters, etc.) multiplied by the daily run time of the display case. The defrost energy consumption is calculated if electric defrost heaters are specified for the candidate case.

The energy savings of a candidate display case are found by comparing the CDEC of the candidate display case with that of similar cases. A baseline CDEC is found by averaging refrigeration load and energy consumption values for all similar cases now being manufactured. As an example, the baseline CDEC is found for the 4-deck meat/deli display cases listed in Table 17. These particular cases use off-cycle defrost, so no defrost heaters are installed.

Candidate cases are then rated by determining the CDEC of each case and comparing the CDEC values to that of the baseline. Table 18 lists the operating specifications for 4 candidate display cases that can be compared to the baseline case described previously. The first two cases in the table are high-efficiency versions of the multi-deck meat/deli case that are now offered by commercial refrigeration manufacturers. The third and fourth cases in the table are prototype display cases now being developed and tested as part of this project. The DOE tested case uses an over-sized evaporator in order to raise the SET of the display case, and a lighting system using efficient T-8 fluorescent bulbs. In the projected final version of the DOE test case, the refrigeration requirement is assumed to be reduced through the use of an improved air curtain 

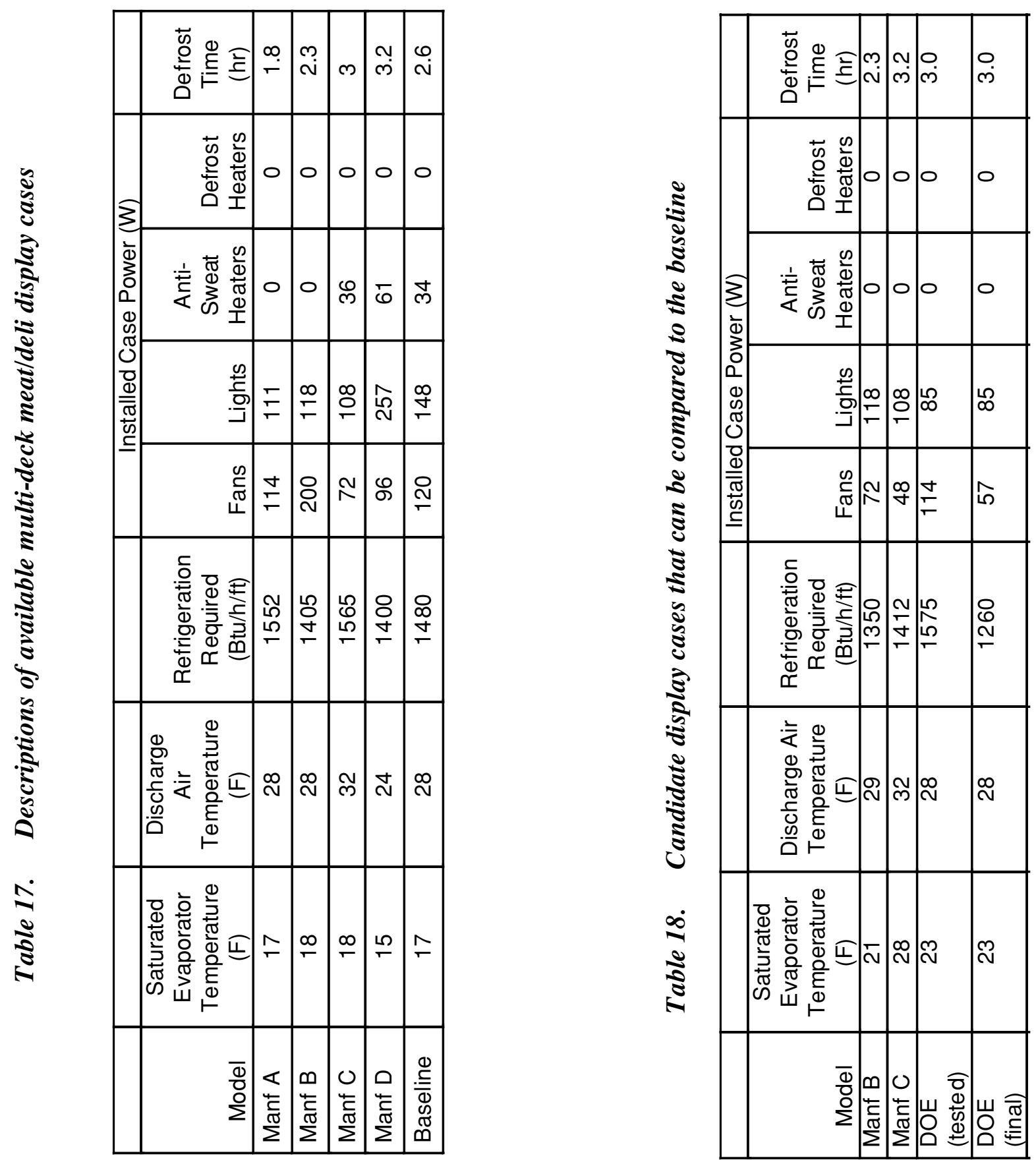
with less ambient air entrainment. ECM fan motors are also installed to reduce the fan power requirement. All candidate cases use off-cycle defrost and have no defrost heaters installed.

Energy savings were found for each of the candidate cases by subtracting their CDEC values from that of the baseline case. Figure 91 shows the results obtained for each of the display cases. The CDEC value for each case is divided into each of its components. The CEC fraction is the largest portion of the energy consumption for all of the display cases examined.

The rating procedure calls for a certain minimum energy savings that must be obtained in order that the case be considered energy efficient. Based upon the results obtained from the EEM modeling and the experimental work presented earlier, a value of 25 percent was chosen as this minimum. Figure 91 shows that 2 of the candidate cases can be considered highly efficient and should be labeled as such.

A possible credit for reduction in display case refrigerant charge was also examined. The credit can be determined through the use of the total equivalent warming impact (TEWI) (13) that will result from the charge reduction. The reduction in atmospheric warming resulting from the charge reduction can be estimated by considering the annual leak rate of refrigerant from the display case. The equivalent carbon dioxide value is found by multiplying the refrigerant leakage by the global warming index for that refrigerant. The reduction in carbon dioxide is seen as the difference in leakage between the candidate and the baseline display cases.

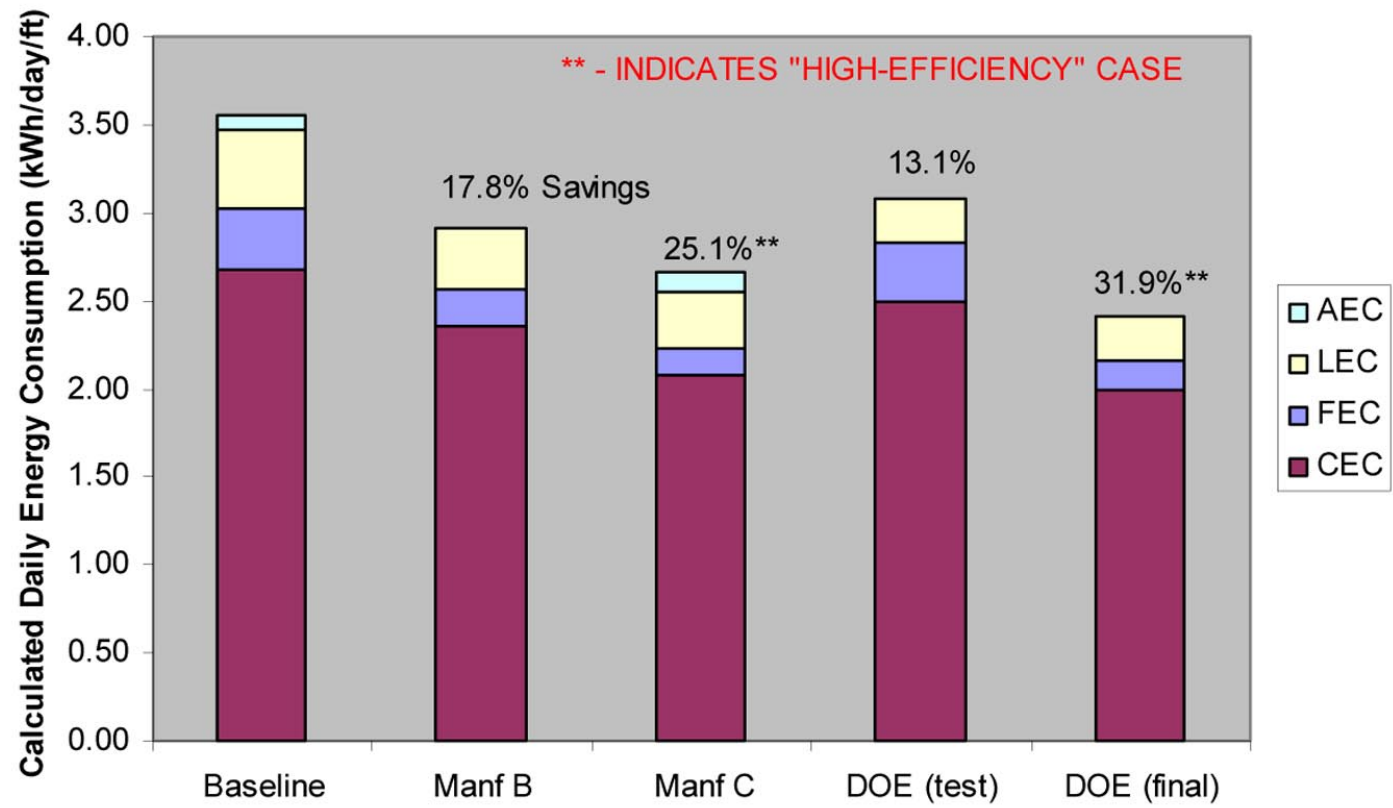

660-DORL-010231-76

Figure 91. Results of CDEC calculation for the baseline and candidate display cases 
The reduction in atmospheric carbon dioxide can then be used to determine an energy savings based upon the $\mathrm{CO}_{2}$ created during electric energy generation. For the United States, approximately $0.65 \mathrm{~kg}$ of $\mathrm{CO}_{2}$ is produced for each $\mathrm{kWh}$ of electric energy generated. Through this value, an energy savings credit can be derived from the refrigerant leakage reduction. The energy savings credit can be added to the savings for the candidate display case.

As an example, a candidate display case might have a charge requirement of $2 \mathrm{lb}$ while the baseline case has a refrigerant charge of $3 \mathrm{lb}$. The savings of $1 \mathrm{lb}$ charge results in a yearly leakage reduction of $0.1 \mathrm{lb}$, assuming an annual leak rate of 10 percent. If the refrigerant is R$404 \mathrm{~A}$, the amount of equivalent carbon dioxide that is not produced each year is $148 \mathrm{~kg}$, or $0.4 \mathrm{~kg} /$ day. Using the relation between electric generation and carbon dioxide production, energy savings of $0.62 \mathrm{kWh}$ /day are found. For a display case that is $12 \mathrm{ft}$ in length, the energy savings from the reduced refrigerant charge would be $0.05 \mathrm{kWh} / \mathrm{day} / \mathrm{ft}$. This amount is subtracted from the CDEC calculated for the candidate case to provide an energy-savings credit for the refrigerant charge reduction. The new $\mathrm{CDEC}$ for the candidate case is then compared to the baseline CDEC in order to determine the energy savings achieved by the candidate case. 


\section{CONCLUSIONS AND RECOMMENDATIONS}

The industry workshops and the Food Marketing Institute survey showed that the supermarket industry has strong interests in developing better open display cases. The survey results showed greatest interest in developing better case air curtains and evaporators, both of which are needed to improve open case performance. The industry is divided over the use of door cases for medium temperature refrigeration. It is realized that the refrigeration load and energy consumption associated with door cases are both significantly lower than seen with open cases; but concerns of possible reduced refrigerated product sales are preventing supermarkets from implementing medium temperature door cases.

A number of near-term technologies were investigated as possible energy efficiency measures (EEMs) for refrigerated display cases. The impact of each EEM on the annual energy consumption of a supermarket was determined through modeling and analysis, using a modified version of DOE-2.3 designed specifically for supermarket simulation. The savings in annual refrigeration energy consumption achieved by each of the EEMs were, in order of greatest impact:

- Door cases for medium temperature applications - 19.5 percent.

- Improved air curtains for open cases - 5.5 percent.

- ECM fan motors - 5.3 percent.

- Scroll cage fans - 1.9 percent.

- Advanced evaporator with improved controls and LSHX - 1.0 percent.

- T-8 fluorescent lights -0.9 percent.

- Modular defrost - 0.2 percent.

Whole-store simulations were also performed in six different locations to compare store energy use with baseline cases and advanced cases employing combinations of EEMs. The first combination consisted of:

- Improved air curtains on all open vertical display cases (assumed 20 percent load reduction).

- Advanced evaporators and controls (all cases).

- $\quad$ Scroll cage fan assembly with ECM fan motors (all cases).

- T-8 fluorescent lights with interior reflective film (all cases). 
- Modular defrost.

The second combination involved the same EEMs except instead of using an air curtain glass doors were implemented on all open vertical cases.

Table 19 summarizes the potential refrigeration energy savings for each location. As might be expected, adding doors to all open cases has a much greater impact than improving air curtains (almost a factor of 2 greater savings projected at all locations). There are, however, institutional barriers to adding doors. Our industry advisors and industry survey results have indicated that there is a significant body of anecdotal evidence that shows shoppers react negatively to doors on meat, deli, and other medium temperature cases. Store merchandizing executives are reluctant to accept doors for these applications for this reason. Therefore, we elected in this project to investigate improvements to air curtain technologies to offer a compromise choice and gain at least some of the load reduction potential.

Table 19. Summary of supermarket refrigeration energy savings estimates from use of advanced display cases (from DOE-2.3 simulations)

\begin{tabular}{|l|c|c|c|}
\hline \multicolumn{1}{|c|}{ Location } & $\begin{array}{c}\text { Baseline Refrigeration } \\
\text { Energy Use } \\
(\mathrm{kWh} / \mathrm{y})\end{array}$ & $\begin{array}{c}\text { Savings with Improved } \\
\text { Air Curtain EEM Package } \\
(\%)\end{array}$ & $\begin{array}{c}\text { Savings with Door EEM } \\
\text { Package } \\
(\%)\end{array}$ \\
\hline Chicago, IL & $1,387,000$ & 14.6 & 27.3 \\
\hline Houston, TX & $1,614,000$ & 12.6 & 25.0 \\
\hline Knoxville, TN & $1,497,000$ & 14.0 & 27.0 \\
\hline Los Angeles, CA & $1,519,000$ & 14.4 & 27.5 \\
\hline Seattle, WA & $1,426,000$ & 15.7 & 28.4 \\
\hline Washington, DC & $1,445,000$ & 14.3 & 27.1 \\
\hline
\end{tabular}

It should be noted that the above analysis was performed for the retrofit of the EEMs in existing display cases with a refrigeration system designed for the original display case load. It is likely that implementation of high-efficiency display cases in a new construction will enable the refrigeration system design capacity to be reduced and thus yield significantly greater annual energy savings than a retrofit case.

The energy savings results for the display case EEMs show that, with the exception of the medium temperature door cases, the savings achieved by individual measures are relatively small. The greatest energy savings are achieved when measures are combined. It is also very likely that changes to the display case air curtain will impact the design of the evaporator and of the case fans. These finding suggest that future project efforts, particularly lab testing and display case prototyping should involve as many EEMs as practical.

The experimental results of DPIV and one-dimensional mini-LDV results are very similar. The DPIV provides a two-dimensional velocity vector field ( $u$ and $v$ ) providing more information of the flow field that is compatible with a two-dimensional CFD simulation. 
Furthermore, the DPIV results provide a measure for calculating the entrainment rate. The LDV is a useful tool for local velocity profiles and is not well suited to obtaining instantaneous global velocity vector fields. However, the LDV provided valuable information that is very consistent with the DPIV results.

The flow field in open, vertical display cases is quite complicated and many factors contribute to the amount of infiltration of warm ambient air into the case. An attempt has been made under this project to identify and quantify the most important parameters that can impact the reduction of the infiltration rate. Basically, three phenomena have the most contribution on the entrainment, turbulence intensity at the DAG, Reynolds number at the DAG (based on the DAG width), and the velocity profile at the DAG (which has an impact on the magnitude of the turbulence intensity). Reducing turbulence intensity at the DAG can potentially reduce the entrainment for Reynolds numbers greater than about 3200. The DAG Reynolds number has a direct effect on the entrainment due to its role in the development of turbulence and mixing in the air curtain flow field. However, it cannot be decreased indiscriminately because of its effects on the food product temperature, and also because sufficient momentum is required to maintain the air curtain integrity (carry the cold air jet) all the way to the RAG. It seems that DAG flow rates between 5.67 to $11.3 \mathrm{~m}^{3} / \mathrm{min}$ (200 to $400 \mathrm{cfm}$ or Reynolds number of 3200 to 5800 ) will generate enough momentum to drive the flow. In summary, it is suggested that a DAG Reynolds number of about 3200 to 3400 should minimize the impacts of turbulence intensity and achieve minimum entrainment rates for the specific case studied. The turbulence intensity at the DAG might also be reduced by manipulation of the velocity profile at the DAG (i.e., trying to generate a laminar profile at the DAG).

Another factor that should certainly be considered is the flow of cold air through the back panel. This flow will stabilize any disturbance to the temperature profile that is caused by intrusion (customers reaching for food), and it fortifies the air curtain strength. Considering the fact that about 70 to 80 percent of this flow will go through the RAG, the question is what is the minimum flow that should be brought in through the back panel to achieve the aforementioned goals? To answer this question, some studies on stabilization of the food product temperature after any intrusion or instability should be performed.

The laboratory performance evaluation of a baseline four-deck open vertical deli display case under the ASHRAE 72-98 Standard procedure revealed that the test results were in close agreement with the manufacturer's published data. Also, the calculated infiltration rate from the test data was in close agreement with infiltration rate estimated from CFD model results.

A draft procedure to rate display cases on the basis of energy-efficiency was developed, using performance testing and energy calculation standards developed by ASHRAE and ARI. The methodology used in the rating procedure establishes a baseline performance for each display case configuration and application based upon published performance data for display cases now manufactured. Energy consumption of the candidate case is then calculated using the same prescribed method, and is compared to the baseline consumption. Candidate cases showing at least 25 percent energy savings are considered "energy-efficient." The 25 percent 
savings criteria was based upon the results achieved through the EEM modeling performed on this project.

Overall, test results indicate that the prototype display case operated more efficiently than the baseline. The energy savings achieved by the prototype case was $6 \mathrm{kWh} / \mathrm{day}$, or 15 percent, however the cooling loads stayed relatively the same for both the prototype and baseline cases.

An increase of 68 percent in evaporator coil UA as well as $34^{\circ} \mathrm{F}$ increase in subcooling (or 7 percent) improved the refrigeration effect of the prototype case by 19 percent. At higher refrigeration effect, the prototype case operated at $6^{\circ} \mathrm{F}$ higher SET than that of the baseline (23 versus $17^{\circ} \mathrm{F}$ ), while achieving the same DAT of $30^{\circ} \mathrm{F}$. Operation of the prototype at higher SET resulted in a decrease of 9 percent in refrigerant mass flow rate. All of these improvements were achieved while the cooling load stayed relatively similar for both the prototype and baseline cases.

The prototype case operated at 8 psig higher suction pressure than that of the baseline, which yielded 10 percent decrease in both the compressor compression ratio and the power usage. Coupling the reduced compressor power and run time resulted in 14 percent decrease in daily compressor energy usage of the prototype case.

Further power savings were made possible through efficient lighting and evaporator fan motor, which produced savings of 21 and 20 percent, respectively. These energy-efficient components also reduced the internal heat gain from 6 to 5 percent of the total refrigeration load.

The overall impacts of all these measures resulted in a 22 percent improvement in overall system EER.

These improvements were achieved while product temperature in both cases stayed the same. The baseline and the prototype cases experienced maximum post-defrost product temperatures of $39.4^{\circ} \mathrm{F}\left( \pm 0.5^{\circ} \mathrm{F}\right)$ and $39.6^{\circ} \mathrm{F}\left( \pm 0.5^{\circ} \mathrm{F}\right)$, respectively, at the bottom shelf front center location.

Despite initial attempts to redesign the DAG to effect reductions in air infiltration, the infiltration load of the prototype display case remained almost the same as that of the baseline (actually 2 percent higher). Further research is highly recommended to improve our understanding of air curtain performance and develop more effective means to reduce infiltration loads on open, vertical display cases. 


\section{REFERENCES}

1. Field Testing of High-Efficiency Supermarket Refrigeration, Foster-Miller, Inc., EPRI report no. TR-100351, Electric Power Research Institute, Palo Alto, CA, December 1992.

2. A.D. Little, Energy savings potential or commercial refrigeration equipment, prepared for Office of Building Technologies, U.S. Department of Energy. Cambridge, MA, June 1996.

3. Walker, D.H. and V.D. Baxter, Field testing of an advanced low-charge supermarket refrigeration system, pp 41-52 in Proceedings of New Technologies in Commercial Refrigeration, P.S. Hrnjak, ed. Conference of International Institute of Refrigeration Commissions B2/D1, Urbana, IL, July 22-23, 2002. ISBN 2-913149-27-8.

4. Faramarzi, R., B.A. Coburn, and R. Sarhadian, "Performance and Energy Impact of Installing Glass Doors on an Open Vertical Deli-Dairy Display Case," ASHRAE Transactions 2002, Vol. 108, Pt 1.

5. Method of Testing Open Refrigerators, ANSI/ASHRAE Standard 72-1998, American Society of Heating Refrigerating and Air-Conditioning Engineers, Inc., Atlanta, GA.

6. Method of Testing Closed Refrigerators, ANSI/ASHRAE Standard 117-2002, American Society of Heating Refrigerating and Air-Conditioning Engineers, Inc., Atlanta, GA.

7. Standard for Commercial Refrigerated Display Cases, ARI Standard 1200-2002, Air Conditioning and Refrigeration Institute, 4100 N. Fairfax Drive, Suite 200, Arlington, VA.

8. Faramarzi, R. 1999. Efficient display case refrigeration. ASHRAE Journal (November):46.

9. Field Testing of Electronically Commutated Motors for Supermarket Display Cases, FosterMiller, Inc., EPRI Report No. TR-106075, Electric Power Research Institute, Palo Alto, CA, December 1995.

10. DOE-2.3 Building energy use and cost analysis program manual, Refrigeration Dictionary, JJH and Associates, Volume 2R, August 2002.

11. Fluent Computational Fluid Dynamics Software, 2003. Fluent, Inc., 10 Cavendish Court, Lebanon, NH 03766-1442.

12. Navaz, H. K., and A. D. Dang, The Development of the Liquid Thrust Chamber Performance (LTCP) Code for Turbulent Two-Phase Flow Combustion of Dense Sprays, Final Report Prepared for NASA/MSFC, Contract No. NAS8-38798, January 1994.

13. Sand, James, Steven K. Fischer, Van D. Baxter, Energy and Global Warming Impacts of HFC Refrigerants and Emerging Technologies, Oak Ridge National Laboratory, sponsored by Alternative Fluorocarbons Environmental Acceptability Study (AFEAS), U.S.

Department of Energy, 1997. 